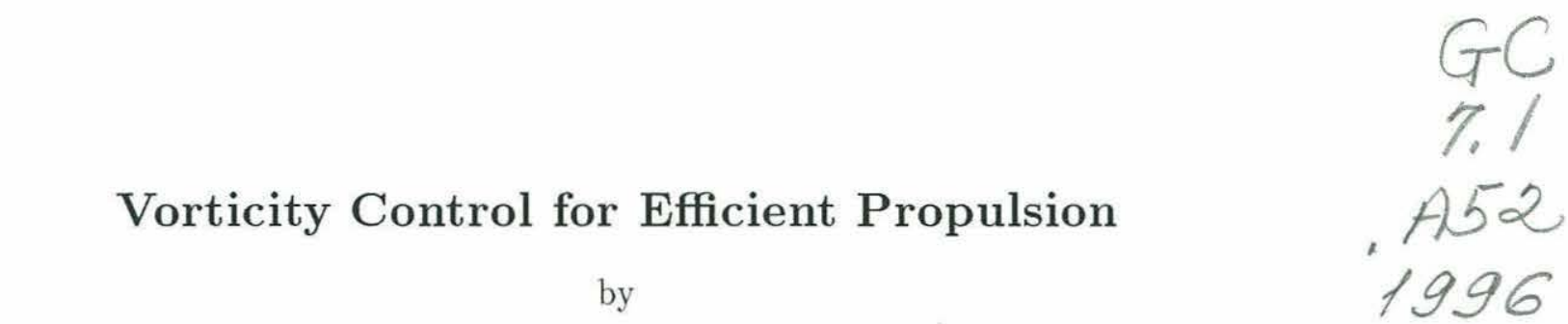

Jamie Marie Anderson

S.M. Ocean Engineering, Massachusetts Institute of Technology/

Woods Hole Oceanographic Institution Joint Program (1992)

B.S. Mechanical Engineering, UC San Diego (1989)

Submitted in partial fulfillment of the

requirements for the degree of

DOCTOR OF PHILOSOPHY IN OCEANOGRAPHIC ENGINEERING

at the

MASSACHUSETTS INSTITUTE OF TECHNOLOGY

and the

WOODS HOLE OCEANOGRAPHIC INSTITUTION

February 1996

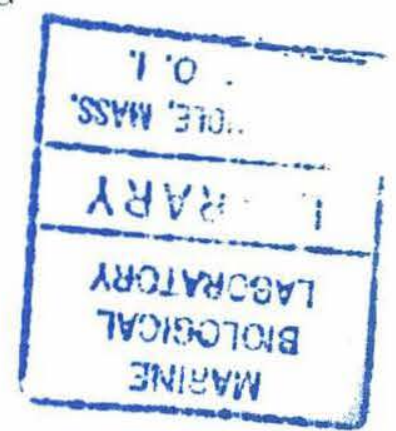

(C) Massachusetts Institute of Technology and

Woods Hole Oceanographic Institution, 1996

All rights reserved.

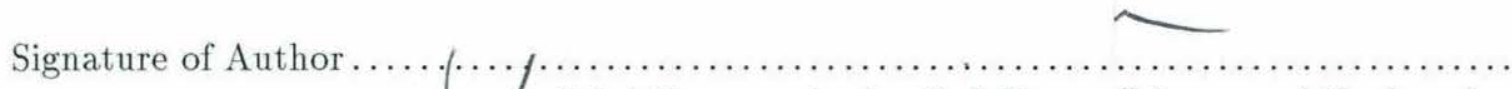
Joint Program in Applied Ocean Science and Engineering Massachusetts Institute of Technology Woods Hole Oceanographic Institution 1. In January 18,1996

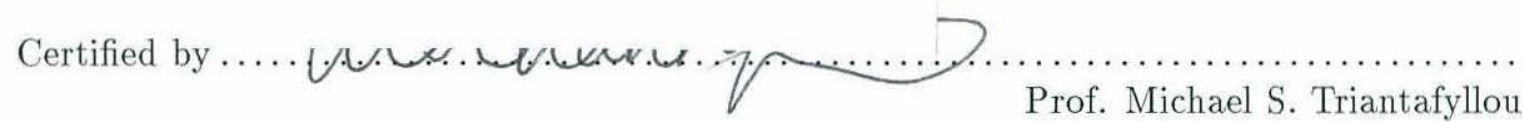
Professor, MIT
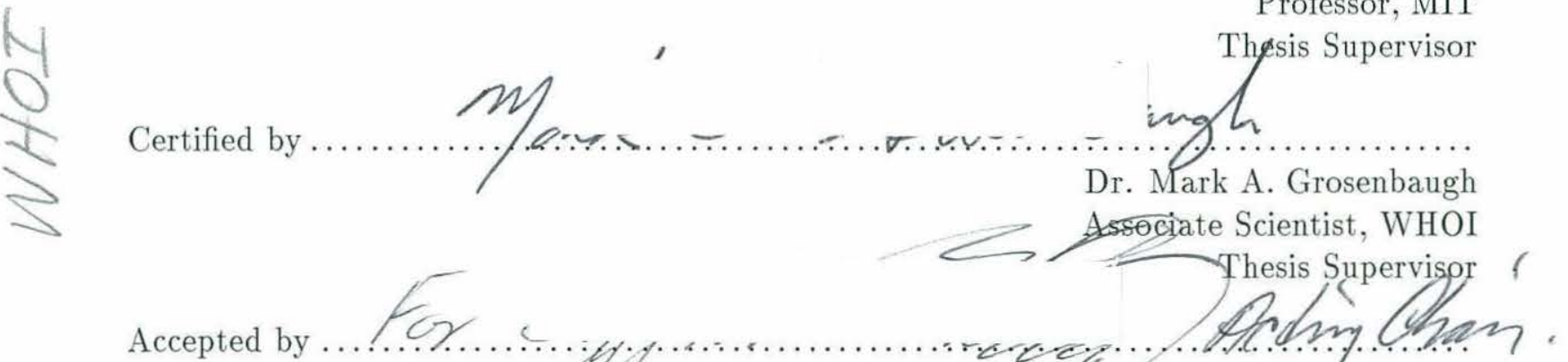

Prof. Arthur B. Baggeroer Massachusetts Institute of Technology/Woods Hole Oceanographic Institution 


\title{
Vorticity Control for Efficient Propulsion
}

by

\author{
Jamie Marie Anderson
}

\author{
Submitted to the Massachusetts Institute of Technology/ Woods Hole Oceanographic Institution \\ Joint Program in Applied Ocean Science and Engineering \\ on January 18,1996 , in partial fulfillment of the requirements \\ for the degree of Doctor of Philosophy
}

\begin{abstract}
Vorticity control is a new paradigm in propulsion hydrodynamics. In this thesis, we study fishlike propulsion strategies as concepts in vorticity control. Our motivation for this research stems from the remarkable capabilities of fish to propel and maneuver in ways presumably optimized by evolution. First, we experimentally measured the flow around a live, naturally swimming fish. We then examined the propulsive properties of a rigid foil which harmonically oscillates in a fish-like manner. Finally, we explored the interaction of a foil with oncoming vorticity to identify the processes by which vorticity can be manipulated. Throughout this thesis, digital particle image velocimetry (DPIV) is used to make quantitative multipoint measurements of the unsteady flow fields.

Fish are the prime example of vorticity control: they propel and maneuver by manipulating vorticity formed along their body which interacts with the tail. Although fish swimming has been studied for several decades, little is known about the details of the flow near the body and its relationship to the propulsive wake. Using DPIV, we measured the flow around a small fish while swimming straight and while turning. In the horizontal plane, the propulsive wave along the body has a dominant influence on the lateral and streamwise velocity components of the flow. Bound vorticity is shed and then favorably affected by the tail motion to produce a thrust wake in the form of a reverse Kármán street. Flow out of the horizontal plane was significant only during aggressive motions such as maneuvering. For straight steady swimming, the flow in the horizontal middle plane closely resembles two-dimensional swimming plate theory.

Next, we investigated the propulsive properties of a rigid flapping foil of chord length $c$, harmonically oscillated in heave and pitch while translating forward. Previous studies indicate that high efficiencies are possible while maintaining large levels of thrust. We explored the flow around and in the wake of a large amplitude flapping foil as a function of frequency and angle of attack. High levels of thrust were achieved for large, $\mathcal{O}(c)$, heave motions and high angles of attack which often exceed the static stall angle. Dynamic stall occurs for most thrust producing cases and its formation and evolution are influenced by the kinematics of the foil. The formation of large stall vortices does not adversely affect efficiency; rather, the dynamic stall vortices are an efficient mechanism by which momentum is transmitted to the wake and can be manipulated to favorably affect the propulsive efficiency.

Finally, we studied the tandem combination of a bluff body and a flapping foil as a simple type of vorticity control to clarify vortex-foil interaction processes. Proper placement of the flapping foil can reposition and/or annihilate undesirable vortices affecting the wake signature and efficiency. Upstream of the foil, a transversely oscillating D-section cylinder was used to produce a Kármán type array of discrete vortices. Foil kinematics and the nature of the encounter with the cylinder vortices were adjusted to identify wake interaction modes. Cylinder vortices merged with same signed trailing and leading edge vortices; or alternatively, strained to disintegration near the foil or merged destructively with the shear layer near the foil trailing edge. Our results indicate that vorticity control of this type may lead to improved efficiency and reduced wake signature.
\end{abstract}

Thesis Supervisors: Prof. Michael S. Triantafyllou, Professor, M.I.T.

Dr. Mark A. Grosenbaugh, Associate Scientist, W.H.O.I. 


\section{Acknowledgements}

Financial support of the Advanced Research Projects Agency, the Office of Naval Research under contracts N00014-92-J-1726 and N00014-94-1-0735, and the Sea Grant Program under Grant Number NA46RG0434 is gratefully acknowledged. In addition, the author was partially supported during her tenure at M.I.T./W.H.O.I as an Office of Naval Research Graduate Fellow.

I would like to specially thank my thesis advisors, Prof. Michael Triantafyllou and Dr. Mark Grosenbaugh for supervising my research and Prof. Dick Yue for serving on my thesis

committee. Dr. Grosenbaugh was particularly helpful in the initial use of the experimental apparatus and the fish swimming experiments.

My successes are largely due to the quiet support of my family and friends. I thank my parents for supporting the choices that I have made in my life. During my time here, my friends and colleagues have made my work enjoyable and meaningful. During the last few years, most of my time was spent at the M.I.T. Ocean Engineering Testing Tank where my colleagues David Barrett, Scott Miller and John Kumph helped me tremendously in preparing and executing my experiments. Séamus Tuohy and Knut Streitlien kept me sane by sharing coffee and lunches at Sam's. Many thanks to Lisa Mitchell for taking in a wayward graduate student and for being such a good friend over the years.

There are so many others who have touched my life during my studies. My heartfelt thanks to all of you. I'd like to specially thank one person who has a special place in my heart. Torsten Berger has been my most patient supporter, my confidant, my best friend and my love. He has been by my side throughout this experience, not always geographically but always emotionally. I thank him for his strength and love. 


\section{Contents}

1 Introduction $\quad 15$

1.1 Motivation . . . . . . . . . . . . . . . . . . . 15

$1.1 .1 \quad$ Propulsive efficiency . . . . . . . . . . . . . . . . . 18

1.2 Chapter preview . . . . . . . . . . . . . . . . . . . 19

2 An experimental method for digital particle image velocimetry near a $\begin{array}{ll}\text { moving body } & 21\end{array}$

2.1 Introduction . . . . . . . . . . . . . . . . . . . 21

2.2 DPIV Algorithm . . . . . . . . . . . . . . . . . . 21

2.3 Implementation issues . . . . . . . . . . . . . . . . . . . . . 24

2.4 Sources of error and calibration . . . . . . . . . . . . 25

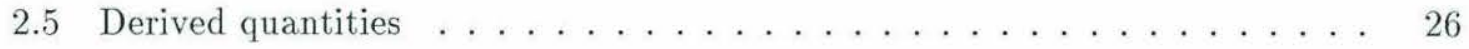

2.6 Experimental apparatus . . . . . . . . . . . . . . . . 27

2.7 Extensions of DPIV method near a moving body . . . . . . . . . . . . 29

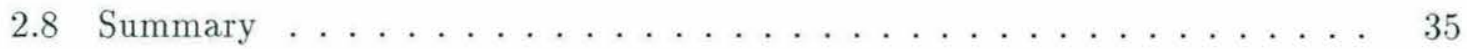

$3 \quad$ Experimental study of fish propulsion and maneuvering $\quad 37$

3.1 Introduction . . . . . . . . . . . . . . . 37

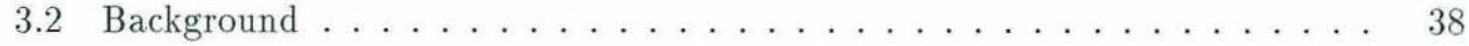

3.3 Flow visualization of swimming fish . . . . . . . . . . . . . . . 41

3.4 Shallow water DPIV experiments on straight swimming fish . . . . . . 45

3.4 .1 Discussion . . . . . . . . . . . . . . . . . . . . . . 48

3.5 Deep water DPIV experiments on straight swimming fish $\ldots \ldots \ldots$. . . . 56

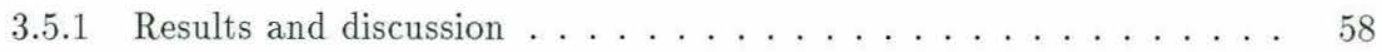

3.6 Kinematic analysis . . . . . . . . . . . . . . . . . . . 67 
3.7 Summary of straight swimming . . . . . . . . . . . . . . 68

3.8 DPIV experiments on maneuvering fish f . . . . . . . . . . 68

3.9 The vertical plane: Three-dimensional effects . . . . . . . . . . . . 79

3.10 Conclusions and recommendations for further research . . . . . . . 80

4 Experiments in flapping foil propulsion $\quad 83$

4.1 Introduction . . . . . . . . . . . . . . . . . 83

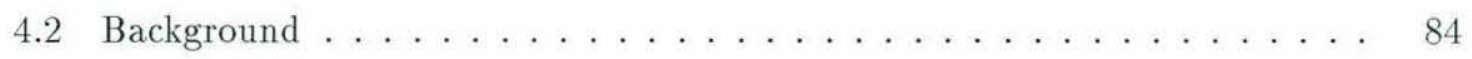

4.2 .1 Visualizations . . . . . . . . . . . . . . . 85

4.2 .2 Dynamic stall research . . . . . . . . . . . . . . . . 86

4.2 .3 Propulsion studies . . . . . . . . . . . . . . . . . 91

4.2.4 Force and efficiency measurements . . . . . . . . . . . 93

4.3 Formulation and definitions $\ldots \ldots \ldots \ldots \ldots \ldots$

4.4 Momentum analysis . . . . . . . . . . . . . . . . . . . . 98

4.5 The experimental system . . . . . . . . . . . . . . . . . . 102

4.6 Flapping foil experiments . . . . . . . . . . . . . . . 105

4.6 .1 Groups I through IV . . . . . . . . . . . . . . . . . . 112

4.6 .2 Groups V and VI . . . . . . . . . . . . . 117

4.6 .3 Groups VII and VIII . . . . . . . . . . . . . . 120

4.6 .4 Group IX . . . . . . . . . . . . . . . . 127

4.7 Discussion . . . . . . . . . . . . . . . . . 136

4.7 .1 Effect of phase angle . . . . . . . . . . . . . . 136

4.7 .2 Thrust force . . . . . . . . . . . . . . . 136

4.7 .3 Wake structure . . . . . . . . . . . . . . . . . 138

4.7 .4 Multiple vortices per cycle . . . . . . . . . . . . . . . 143

4.8 Conclusions . . . . . . . . . . . . . . . . . . . . . . . . . . . . 144

5 Vorticity control for enhanced propulsive efficiency $\quad 147$

5.1 Introduction . . . . . . . . . . . . . . . . . . . . 147

5.2 Background . . . . . . . . . . . . . . . . . . 147

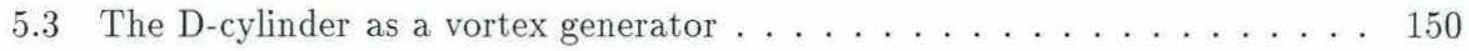

5.3 .1 Definitions . . . . . . . . . . . . . . . . . . 151

5.4 Cylinder-foil tandem arrangements . . . . . . . . . . . . . 157 
5.4 .1 Results and discussion . . . . . . . . . . . . . . 162

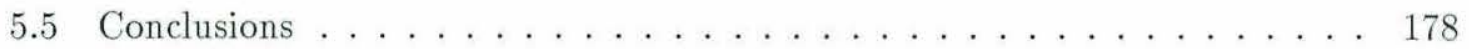

6 Conclusions 181

6.1 Summary . . . . . . . . . . . . . . . . . . . . . 181

6.2 Recommendations for further research . . . . . . . . . . . . . . . . . . 182

$\begin{array}{ll}\text { References } & 185\end{array}$ 


\section{List of Figures}

1-1 The Kármán vortex street behind a circular cylinder . . . . . . . . . . 16

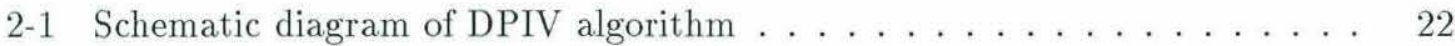

2-2 DPIV experimental apparatus . . . . . . . . . . . . . . 28

2-3 Schematic diagram of near-body DPIV algorithm . . . . . . . . . 30

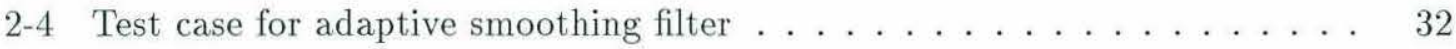

2-5 Artificial cylinder image used to verify near-body DPIV method . . . . . 33

2-6 Two artificial near body test cases . . . . . . . . . . . . . . . 34

3-1 Two and three-dimensional fish swimming theories . . . . . . . . . 39

3-2 Photographic sequence of Pearl Danio visualization . . . . . . . . . . . . . 42

3-3 Giant Danio morphology . . . . . . . . . . . . . . . . . 42

3-4 K-fluid visualizations of straight swimming fish . . . . . . . . . . 44

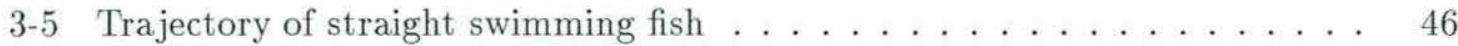

3-6 Velocity field in the wake of a swimming fish in shallow water . . . . . . . 47

3-7 Vorticity field in the wake of a fish swimming in shallow water . . . . . 47

3-8 Velocity field in the wake of a swimming fish in shallow water. . . . . . . 49

3-9 Vorticity field in the wake of a fish swimming in shallow water . . . . . . 49

3-10 Velocity field in the wake of a swimming fish in shallow water. . . . . . . . 50

3-11 Vorticity field in the wake of a fish swimming in shallow water . . . . . 5 50

3-12 Velocity field in the wake of a swimming fish in shallow water. . . . . . . 51

3-13 Vorticity field in the wake of a fish swimming in shallow water . . . . . 51

3-14 Velocity field in the wake of a swimming fish in shallow water. . . . . . . . 52

3-15 Vorticity field in the wake of a fish swimming in shallow water . . . . . . 52

3-16 Velocity field in the wake of a swimming fish in shallow water. . . . . . . 5 53

3-17 Vorticity field in the wake of a fish swimming in shallow water . . . . . . 53 
3-18 Summary of observed flow around a straight swimming fish . . . . . . . . 54

3-19 Flow around and in the wake of a small swimming eel . . . . . . . . . 57

3-20 Flow near a travelling wave moving from left to right. . . . . . . . . 57

$3-21$ Visualization of flow near a waving plate . . . . . . . . . 58

3-22 Velocity field in the wake of a swimming fish, semi-deep water. . . . . . . 60

3-23 Vorticity field in the wake of a swimming fish, semi-deep water. . . . . . . . 60

3-24 Velocity field in the wake of a swimming fish, semi-deep water. . . . . . . . 61

3-25 Vorticity field in the wake of a swimming fish, semi-deep water. . . . . . . . 61

3-26 Velocity field in the wake of a swimming fish, semi-deep water. . . . . . . . 62

3-27 Vorticity field in the wake of a swimming fish, semi-deep water. . . . . . . . 62

3-28 Velocity field in the wake of a swimming fish, semi-deep water. . . . . . . . 63

3-29 Vorticity field in the wake of a swimming fish, semi-deep water. . . . . . . . 63

3-30 Velocity field in the wake of a swimming fish, semi-deep water. . . . . . . . 64

3-31 Vorticity field in the wake of a swimming fish, semi-deep water. . . . . . . . 64

$3-32$ Magnification of velocity field near body . . . . . . . . . . . . . 65

3-33 Summary of flow around a straight swimming fish in semi-deep water . . . 66

3-34 Three-dimensional flow structure around a straight swimming fish $\ldots \ldots$. . 69

3-35 Trajectory of maneuvering fish . . . . . . . . . . . . 71

3-36 Velocity field in the wake of a turning fish, starting position. . . . . . . 72

$3-37$ Velocity field in the wake of a turning fish. . . . . . . . . . . 73

3-38 Velocity field in the wake of a turning fish. . . . . . . . . . . . . 74

3-39 Velocity field in the wake of a turning fish . . . . . . . . . . . . 75

3-40 Velocity field in the wake of a turning fish . . . . . . . . . . . 76

$3-41$ Velocity field in the wake of a turning fish. . . . . . . . . . . 77

$3-42$ Vorticity field in the wake of a turning fish . . . . . . . . . . . 78

$3-43$ Flow structure around a turning fish . . . . . . . . . . . . . . 79

4-1 Experimental thrust coefficient as a function of angle of attack and Strouhal

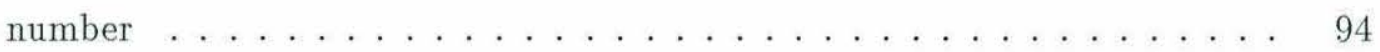

4-2 Experimental efficiency as a function of angle of attack and Strouhal number 95

4 -3 Definitions of foil motion . . . . . . . . . . . . . . . 97

4-4 Kinematic relationships for flapping foil . . . . . . . . . . . . . 97 
4-5 Importance of angle of attack phase . . . . . . . . . . . . . . . . 99

$4-6$ Control volume for momentum analysis . . . . . . . . . . . . . . 100

$4-7 \quad$ Visualization and DPIV tank . . . . . . . . . . . . 103

4-8 Examples of angle of attack profiles . . . . . . . . . . . . . 107

4-9 Angle of attack profile types as a function of maximum angle of attack, trailing edge Strouhal number and pitch amplitude . . . . . . . . . . 108

4-10 DPIV velocity data: case fa05 . . . . . . . . . . . . . . 113

4-11 DPIV velocity data: case fa03 . . . . . . . . . . . . . . . . . 114

4-12 DPIV velocity data: case fa08 . . . . . . . . . . . . . . . . . 114

4-13 DPIV velocity data: case fa04 . . . . . . . . . . . . . . 115

4-14 DPIV velocity data: case $\mathrm{fa} 02 \ldots \ldots \ldots \ldots \ldots \ldots$

4-15 DPIV velocity data: case fa07 . . . . . . . . . . . . . 117

4-16 DPIV velocity data: case fa10 . . . . . . . . . . . . . . 118

4-17 DPIV velocity data: case fa11 . . . . . . . . . . . . . . . 118

4-18 DPIV velocity data: case fa12 . . . . . . . . . . . . . . . . 119

4-19 DPIV velocity data: case fa13 . . . . . . . . . . . . . . 120

4-20 DPIV velocity data: case fa14 . . . . . . . . . . . . . . . . 121

4-21 DPIV velocity data: case fa15 . . . . . . . . . . . . . . . . . 121

4-22 Foil trajectories and angle of attack phase relationship as a function of phase angle . . . . . . . . . . . . . . . . . . . 123

4-23 DPIV velocity data: case fa19 . . . . . . . . . . . . . . . . 124

4-24 DPIV velocity data: case fa20 . . . . . . . . . . . . . . . . 124

4-25 DPIV velocity data: case fa21 . . . . . . . . . . . . 125

4-26 DPIV velocity data: case fa34 . . . . . . . . . . . . . . 125

4-27 DPIV velocity data: case fa33 . . . . . . . . . . . . . 126

4-28 DPIV velocity data: case fa22 . . . . . . . . . . . . . . 126

4-29 DPIV velocity data: case fa23 . . . . . . . . . . . . . 127

4-30 DPIV velocity data: case fa24 . . . . . . . . . . . . . . . . 128

4-31 DPIV velocity data: case fa25 . . . . . . . . . . . . . . . . 128

4-32 DPIV velocity data: case fa30 . . . . . . . . . . . . . . 129

4-33 DPIV velocity data: case fa32 . . . . . . . . . . . . . . 129

4 -34 DPIV velocity data: case fa26 . . . . . . . . . . . . . 130 


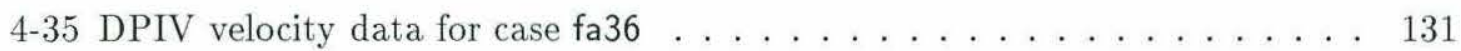

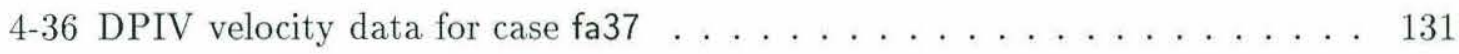

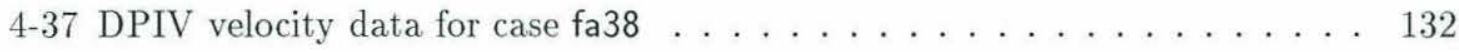

4-38 Leading edge vortex development sequence for fa36 . . . . . . . . 133

4-39 Leading edge vortex development sequence for fa37 . . . . . . . . . . 134

4-40 Leading edge vortex development sequence for fa38 f . . . . . . . . 135

4-41 Thrust coefficient variation with phase angle $\ldots \ldots \ldots \ldots \ldots \ldots$

4-42 Wake width variation with phase angle $\ldots \ldots \ldots \ldots \ldots \ldots$

$4-43$ Stall vortex location as a function of phase angle $\ldots \ldots \ldots \ldots \ldots$

4-44 Experimental thrust coefficient results comparison . . . . . . . . . 139

4-45 Wake patterns as a function of Strouhal number and angle of attack . . . 142

5-1 Wake interaction modes . . . . . . . . . . . . . . . . . . 149

5-2 Schematic diagram of D-cylinder motion and wake structure . . . . . 153

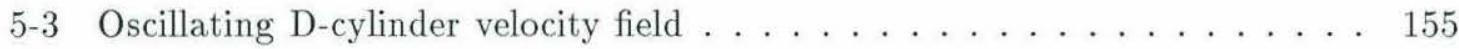

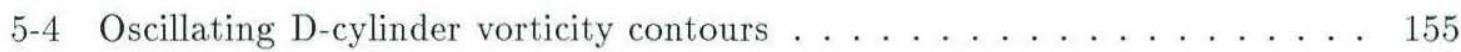

5-5 Performance summary of oscillating D-cylinder $\ldots \ldots \ldots \ldots \ldots \ldots$

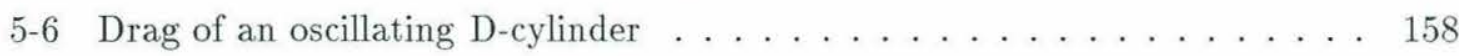

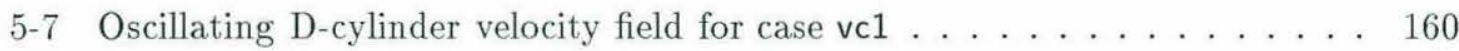

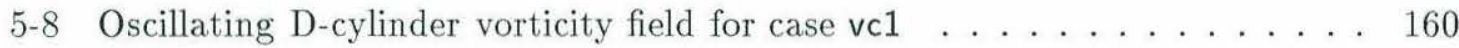

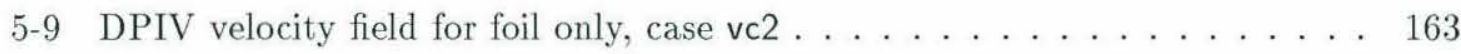

5-10 DPIV vorticity field for foil only, case vc2 $\ldots \ldots \ldots \ldots \ldots \ldots$

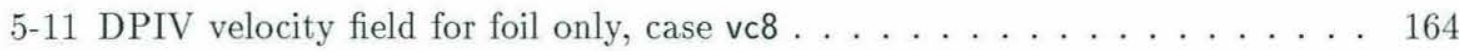

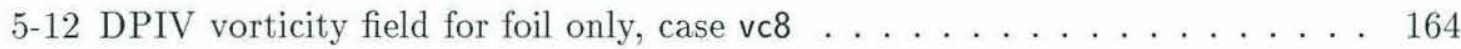

5-13 Flow summary for vc3 case $\ldots \ldots \ldots \ldots \ldots \ldots \ldots$

5-14 Flow summary for vc4 case $\ldots \ldots \ldots \ldots \ldots \ldots \ldots \ldots$

5-15 Flow summary for vc5 case $\ldots \ldots \ldots \ldots \ldots \ldots$

5-16 Flow summary for vc6 case $\ldots \ldots \ldots \ldots \ldots \ldots \ldots \ldots \ldots$

5-17 Flow summary for vc7 case $\ldots \ldots \ldots \ldots \ldots \ldots \ldots \ldots \ldots \ldots \ldots$

5-18 Flow summary for vc9 case $\ldots \ldots \ldots \ldots \ldots \ldots \ldots \ldots$

5-19 Flow summary for vc10 case $\ldots \ldots \ldots \ldots \ldots \ldots \ldots \ldots \ldots$

$5-20$ Flow summary for vc11 case $\ldots \ldots \ldots \ldots \ldots \ldots \ldots \ldots$ 
5 -21 Flow summary for vc12 case . . . . . . . . . . . . . . . 177

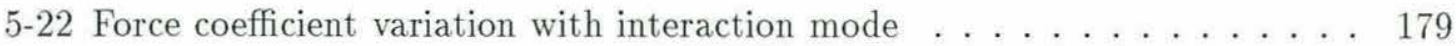




\section{List of Tables}

3.1 Summary of fish wake analysis . . . . . . . . . . . . . 67

4.1 Summary of foil experiments cases . . . . . . . . . . . . . 110

4.2 Summary of foil experiments cases and results . . . . . . . . . . . . . 111

4.3 Comparison of force coefficient results with M.I.T. data . . . . . . . 140

5.1 Summary of D-cylinder experiments and results . . . . . . . . . . . . 153

5.2 Vortex control experiments . . . . . . . . . . . . . 161 


\section{Chapter 1}

\section{Introduction}

\subsection{Motivation}

Vorticity control is defined as active and purposeful generation and repositioning of vorticity. In this thesis, we study the role of vorticity in propulsive wakes and ways in which efficiency can be affected by controlling the placement of vorticity. Vorticity generation in propulsion is generally regarded as an undesirable consequence of jet production. Even for the case of a simple two-dimensional jet, the shear layer is unstable and eventually will break down into discrete regions of vorticity. This view fails to consider the fact that jet generation is intrinsically related to vorticity generation, which may undergo dynamical changes, resulting in the formation of large vortical patterns. Hence, redistribution of vorticity which is often an unavoidable consequence of the instability of the jet flow, is the cause of the appearance of large vortices. The contribution of this thesis is to show that such vorticity redistribution may result in high propulsive efficiency.

We begin by addressing methods by which a swimming fish generates thrust. Fish swimming hydrodynamics has captured the interest of many noteworthy researchers for centuries. $10^{9}$ years of evolution have produced more than 22000 species of fishes which are "masters of the art of propulsive interaction with water" ${ }^{1}$. Despite remarkable individual species adaptations, most fishes swim using similar motions. Consequently, it is well accepted that fishes have evolved to efficiently propel themselves through water.

It has been known for many decades that fishes produce a highly vortical jet wake. Kármán and Burgers [126] first introduced the notion that the famous Kármán street shown

\footnotetext{
${ }^{1}$ Videler [123]
} 


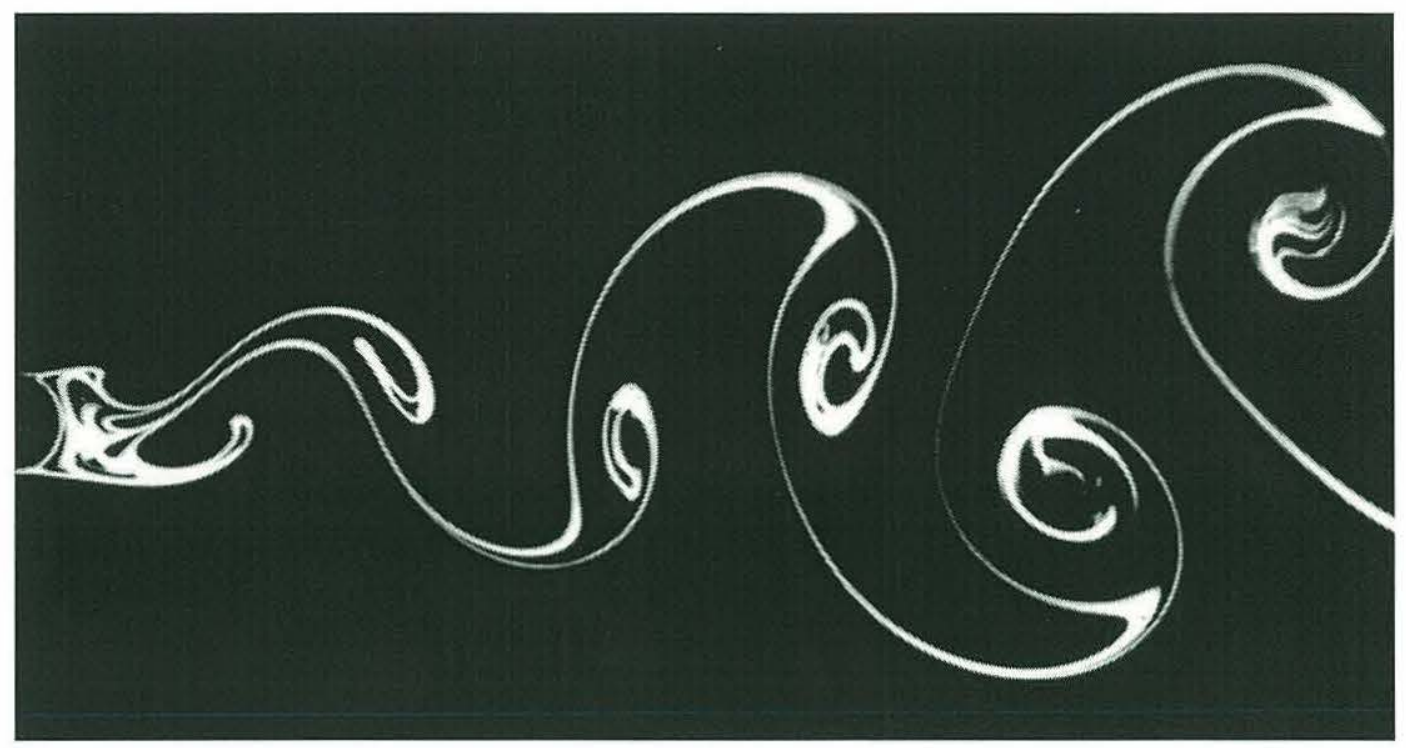

Figure 1-1: The Kármán vortex street behind a circular cylinder at $R_{d}=140$. Photograph by S. Taneda, from Van Dyke (1982)

in Figure 1-1 can also work in reverse, generating thrust instead of drag. Lighthill [64] remarks that the nature of these thrust producing wakes have been "hitherto neglected" by most researchers. The conventional understanding of these wakes is that the vortices arrange in vortex rings which are efficient packages for energy and momentum to be transferred to the wake. Our experimental work with live swimming fish shows that the structure of the vortex wake is inherently linked to the undulation of the fish body as well as the movement of the tail. The formation of discrete vortical structures is not a secondary effect, rather, the fish forces the wake to be highly vortical at a frequency which produces the most thrust for minimum input.

Flapping foil propulsion, the engineering application of fish-like movement of a hydrofoil, has received attention in recent decades as a possible competitor for propellers. High efficiencies, comparable to and exceeding those of typical propellers, have been reported in the literature which have sparked our interest. We define flapping motion as the simultaneous heaving and pitching of a foil while translating forward. Large amplitude foils oscillated at the correct frequency can provide significant thrust levels very efficiently. The premise has many exciting engineering applications such as ship propulsion [145], maneuvering and flow control. One obvious advantage over a conventional propeller is that the effective propul- 
sor area can be increased to provide power more efficiently. For instance, a flapping foil propulsor could more fully utilize the beam of a ship.

In this thesis we systematically examine the flapping foil parameter space to explore the vortical wake structure and its relation to propulsive efficiency as measured by other researchers. Previous efforts in this area are limited to flow visualization and lightly loaded foils operating at low angles of attack. Here, we focus on heavily loaded foils operating at moderately large angles of attack (often in excess of the stall angle) in order to achieve thrust forces appropriate for engineering application. Using digital particle image velocimetry, we are able to explore the multi-dimensional parameter space with minimal experimental and computational effort as compared to traditional flow measurement techniques. Our results demonstrate that high thrust, high efficiency motions are dominated by the process of dynamic stall which is controlled by the foil kinematics. Kinematic manipulation of the stall process can favorably affect the efficiency of the motion by controlling the generation and placement of vorticity.

Our final topic in this work is the notion of vorticity control: the manipulation of vorticity to favorably affect the flow. There are many contexts in which vorticity control can be applied. In aircraft design for instance, several types of passive devices are used currently to adjust the flow by injecting or managing vorticity. For example, vortex generators are small shedding devices used on aircraft wings to inject vorticity into the boundary layer in order to delay separation. Another example of passive vorticity control is the use of a splitter plate behind a translating cylindrical section. The plate prevents the formation of the Kármán street, reducing the oscillatory forces on the cylinder.

Active vorticity control which utilizes flow sensors and feedback control techniques offers obvious advantages over passive flow control devices. Unpredictable, unsteady disturbances can be measured and then cancelled or repositioned, altering the hydrodynamic load and reducing the wake signature. In this thesis we investigate the interaction processes between vorticity shed from a bluff body and a thrust producing flapping foil. Vorticity control is achieved in an open loop sense: the kinematics and geometry of the tandem arrangement control the encounter and evolution of vorticity. We demonstrate effective cancellation of a strong Kármán street drag wake and relate the overall performance to the nature of the vortex interactions. 


\subsubsection{Propulsive efficiency}

Our primary motivation throughout this thesis is the efficient generation of thrust. In this introductory section, we present a classic actuator model by which we can compare propulsion strategies.

Conventional propellers are universally used in all types of marine vehicles from small submersibles to the largest freight ships. Screw propellers are unrivaled as propulsors for several reasons. Propellers deliver high levels of variable thrust in a single motor driven shaft. Reversing thrust is generated simply by reversing the shaft rotation. Recovery of the ship wake improves the efficiency by increasing momentum input to the propeller.

However, there are also disadvantages in propeller propulsion. As in all propulsors, the highest thrust is produced at low advance ratio where the efficiency is near zero. Typically, the highest efficiency occurs when the thrust coefficient is reduced by approximately $50 \%$ of its bollard value. Hence, there is a trade-off between efficiency and thrust level. It is not practical to operate large propellers at low frequencies as this requires large motors. Cavitation of the propeller is also an issue at high shaft speeds. Further, there is loss of momentum in the wake due to the presence of stable helical vortices which rotate the wake about an axis along the direction of motion. This rotational energy is wasted as it does nothing to advance the body. Contrarotating propellers recover some of this lost momentum but involve more complex mechanical system.

Propulsor effectiveness can be evaluated with a simplified momentum analysis by modelling the propulsor as an actuator disk. The actuator disk imparts a sudden increase in pressure to the fluid passing through it which uniformly accelerates the flow, generating thrust evenly distributed over the disk area. Such a device likely does not exist, however, the simple actuator model provides a basis for comparison of different propulsors.

If the total thrust produced is $T$, the nondimensional thrust coefficient is

$$
C_{T}=\frac{T}{\frac{1}{2} \rho A_{\circ} U^{2}}
$$

where $\rho$ is the fluid density, $A_{\circ}$ is the disk area (area "swept" by the actuator) and $U$ is the flow velocity into the propulsor. The ideal efficiency can be calculated using momentum 
and energy arguments to be [91]

$$
\eta_{I}=\frac{\text { useful work obtained }}{\text { work expended }}=\frac{2}{1+\left(C_{T}+1\right)^{\frac{1}{2}}}
$$

Equation (1.2) relates the trade-off relationship between thrust coefficient and efficiency. As the thrust coefficient approaches zero, the ideal efficiency tends towards unity. Similarly, as the thrust increases, the efficiency is diminished. Equation (1.2) also shows how efficiency relates to the swept area of the actuator disk. As the disk area increases, $C_{T}$ decreases which improves the efficiency. An ideal efficient actuator should operate over a large swept area with low thrust levels. Large amplitude flapping foils sweep a large disk area $2 A_{T E} s$ where $A_{T E}$ is the trailing edge excursion and $s$ is the span. Thus, within the context of simple actuator disk theory, flapping foils are worthy competitors for conventional propellers.

\subsection{Chapter preview}

In Chapter 2, we summarize the experimental method of digital particle image velocimetry (DPIV) used throughout this thesis. DPIV provides multipoint real-time velocity measurements within a planar slice of the flow. Special adaptations to the standard method are implemented to correct the flow near boundaries. Details of the method and related derived quantities are discussed in detail.

In Chapter 3, we look at the flow around a swimming fish. Kalliroscopic visualizations and DPIV are used to reveal the flow along the body and in the wake of a live swimming fish. Measurements for straight swimming and maneuvering are presented in shallow and deep water.

Chapter 4 is the focal point of this work. Here, we explore the flow mechanisms involved in flapping foil propulsion. An array of experiments spanning the parameter space are presented which show thrust development and its relationship to wake structure and evolution. Comparisons are made with force and efficiency measurements to assess the efficiency of flapping foil propulsors and the relationship to wake structure.

In Chapter 5, we study the problem of wake energy recovery and vortex cancellation

using a flapping foil to manipulate vortices shed by an oscillating cylinder. A variety of interaction modes are identified which depend on the phase relationships between wake vortices and the kinematics of the flapping foil. 
Finally, in Chapter 6 we summarize the conclusions and contributions of this thesis and make recommendations for further research. 


\section{Chapter 2}

\section{An experimental method for digital particle image velocimetry near a moving body}

\section{$2.1 \quad$ Introduction}

In this chapter we describe our experimental methods which implement digital particle image velocimetry (DPIV) for flow measurement and visualization. Unlike other methods, with DPIV we can measure an entire two-dimensional velocity field at one instant in time,

with simultaneous flow visualization. Using DPIV, one experiment is required to map an unsteady flow, whereas with other single point methods, we would have to do many experiments with many probes and considerable post-processing to accomplish the same goal.

In the following sections, we present the general DPIV method, its advantages and limitations, the technical details of the experimental method and special implementations near moving boundaries.

\subsection{DPIV Algorithm}

The DPIV method derives from the analog flow measurement techniques of laser speckle velocimetry (LSV) and particle image velocimetry (PIV). In both methods, the flow is seeded with small light reflecting particles and a plane is illuminated using a flash lamp or laser sheet. Double exposed photographs of the particles in a plane are taken which reveal speckles (LSV) or individual particle traces (PIV). The photographs are then mechanically interrogated with a diffracted laser beam to produce what are known as Young's fringes. 


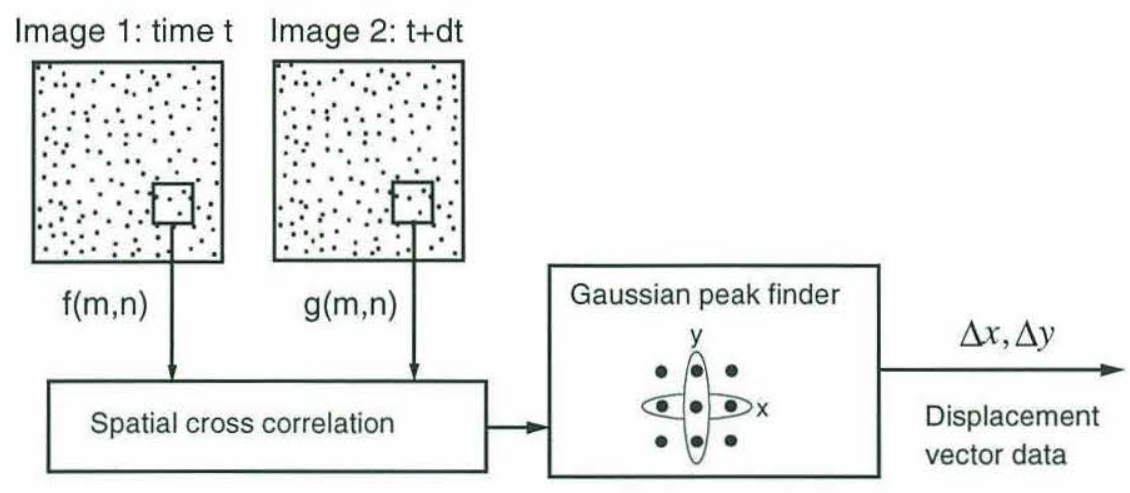

Figure 2-1: Schematic diagram of DPIV algorithm

The spacing and angle of these fringes correspond to the displacement and direction of the particle movements. Adrian [2] gives an excellent review of these two methods.

LSV and PIV have been applied in many successful fluids experiments; however, they are not practical for many reasons. Mechanically interrogating the images is tedious and time consuming. The required double exposures give no phase information which makes reversing flows difficult to study. Particle images which overlap one another also give no information. Digital particle image velocimetry solves these problems while using a completely digital implementation. First introduced by Willert and Gharib [135, 136], DPIV uses single exposed images and a spatial cross correlation calculation between the images to track particle displacements.

The basic algorithm is outlined in Figure 2-1. Image data from two successive video frames are collected and stored digitally in levels of greyscale. In each of the frames, we examine small interrogation regions at the same spatial position. The data in these interrogation windows, $f(m, n)$ and $g(m, n)$, are used to compute the cross correlation function,

$$
\Phi_{f g}=f(m, n) \circ g(m, n)=\sum_{k=-\frac{N}{2}}^{\frac{N}{2}-1} \sum_{l=-\frac{N}{2}}^{\frac{N}{2}-1} f(k, l) g(k+m, l+n)
$$

where $m$ and $n$ refer to individual pixels. $N$ is the interrogation window size in pixels.

Cross correlation is a measure of "goodness of match" between images. If the cross correlation function has a strong narrow peak, the first image matches the second image 
when the first image is laid over the second at the location of the peak. The correlation peak locates the average displacement of the sampled group of particles.

We implement the cross correlation calculation in the Fourier domain, replacing the convolution with complex conjugate pointwise multiplication of the two-dimensional Fourier transforms. This is known as the correlation theorem

$$
f(m, n) \circ g(m, n) \Leftrightarrow F(u, v) G^{*}(u, v)
$$

The order in which the correlation is performed is important in DPIV to preserve phase information between the two images. Implementation of the algorithm with Fast Fourier Transforms (FFTs) limits the allowable displacement between the two images to $\pm \frac{N}{2}$ pixels. This is due to the periodicity of the Fourier transform at the sample frequency and is formally known as the Nyquist criterion. For a sample of size $N$, the maximum resolvable displacement without any overlap in the Fourier domain (i.e., aliasing) is $\pm \frac{N}{2}$ pixels. In principle, we would like the cross correlation calculation to check for a match anywhere in the second video frame. However, we choose to limit our search spatially to gain computational speed by using Fourier methods. Hence, only the image data inside the interrogation window are used to compute each displacement. A weighting function is not used along the edges of the window because that would bias the correlation peak towards the center of the window.

After the cross correlation is computed, the peak must be located with sub-pixel accuracy in order to derive displacement data with low relative errors. For example, a displacement error as little as half a pixel can produce a velocity error on the order of $0.5 \frac{\mathrm{cm}}{\mathrm{s}}$ for typical video frame rates and much greater for faster framing rates. Accurate and computationally economical peak estimation is required to achieve sub-pixel resolution. The algorithm uses a Gaussian curve fit to the data around the maximum correlation value in both image directions independently. The fit has the form

$$
p(\zeta)=c_{1} e^{\frac{-\left(\zeta-c_{2}\right)^{2}}{c_{3}}}
$$

where $\zeta$ is the spatial coordinate. Four data points in addition to the maximum are used to make the fit as indicated by the circled points in the peak finder box shown in Figure 2-1. The Gaussian fit models the peak well, with best results when the peak is broad. The success of the Gaussian model is not surprising since particle image intensity distributions 
are Gaussian. This technique works well only when the particle images occupy multiple pixels. Lourenco and Krothapalli [65] also report excellent results with Gaussian curve fits.

\subsection{Implementation issues}

For the DPIV algorithm to work well, several criteria must be met. First, there must be adequate particle seeding. The algorithm requires that the seeding is randomly distributed over the entire image plane. Gaps in the seeding density can locally bias the correlation computation and should be avoided. The camera, lens and image plane must be adjusted so that the particle images are a few (2-3) pixels in size. Also, there should be ten or more distinct particles in each interrogation window. Willert and Gharib [136] systematically studied the seeding requirement and found that 10-20 particles per window are sufficient to ensure good correlations. Seeding density higher than 20 particles per interrogation window showed no improvement in the calculation.

The implementation of the algorithm using FFTs requires that the maximum average displacement anywhere in the flow is less than half the interrogation window size (Nyquist limit). The objective is to choose the interrogation window size to be as small as possible without violating the Nyquist limit and the seeding requirement. In practice, the rule of thumb is to limit the maximum allowable displacement to one third of the window size.

The number of independent measurements (i.e., correlations) in a given image is the total number of interrogation windows which fit into the image without overlap. For instance, a $768 \times 480$ pixel image with $32 \times 32$ pixel interrogation windows gives 360 independent displacement measurements. Oversampling of the data is possible by overlapping the interrogation windows, that is, stepping the window in increments less than the window size. For example, a $768 \times 480$ pixel image sampled with $32 \times 32$ pixel windows stepped in 8 pixel intervals, gives 5760 measurements. These added measurements are not truly independent since no new image data are used. However, they increase the spatial resolution of the data. The mean particle separation limits the allowable step size since below that limit, no new particles would be included in the correlation calculation.

The displacement data are post-processed to remove outliers and high frequency jitter inherent to the algorithm. Outliers are identified by comparing the displacement magnitude at a given point with the values of the eight nearest neighboring points. If the value 
differs from at least four of its eight neighbors by some threshold value, that point is reinterpolated using linear interpolation with the neighboring points. Then, the corrected data are filtered by replacing each data point with a weighted average of the data point and its eight neighbors. This filter does not remove any information if the data are sufficiently oversampled to begin with, that is, the step size is at most, half of the interrogation window size.

\subsection{Sources of error and calibration}

DPIV is subject to many sources of error, both experimental and computational. The factors which contribute most to the displacement error are particle image size, interrogation window size, seeding density and flow gradients within the interrogation window. There must be enough particles inside the window in order to get a sharp correlation peak that is robust to noise from loss of particles out of the image plane and out of the interrogation window. The window cannot be arbitrarily small because smaller windows contain a larger fraction of particles truncated by the window edge which is a large source of error. Background image noise will reduce the correlation peak and spatial gradients inside the window will broaden the peak. Other lesser sources of error include instrument performance characteristics, quantization effects and computational error.

The windowing process in DPIV filters out spatial frequencies smaller than the interrogation window size. This is a problem when the flow field of interest has fine scale motions and flow reversals such as in a vortex core. To correctly resolve such fine scale features, the image view should be enlarged in the region of interest by moving the camera or by reducing the window size. Willert and Gharib [136] show that there is approximately $70 \%$ attenuation of 32 pixel features when the data are processed with $16 \times 16$ pixel interrogation windows. One should always loosely interpret displacement data for scales less than twice the window size.

Willert and Gharib [136] systematically examined the processing error in the DPIV system (both hardware and computation) on an actual image displaced on a linear motion table. They first looked at a stationary image and found a statistical error of 0.01 to 0.03 pixels. They then studied image shifts from 0.045 to 9 pixels and obtained errors ranging between 0.01 to 0.15 pixels respectively. At the lowest shifts, the relative error was around 
$25 \%$ whereas at larger displacements, the error was less than $2 \%$. Hence, the time between images should be adjusted such that the particle displacements are as large as possible without violating the Nyquist limit. Quantization effects and numerical noise were found to be insignificant compared to seeding and window sizing effects on the measurement error.

We calibrated our hardware and processing techniques in the same manner as Willert and Gharib using an artificially generated test image displaced with a rotary table instead of a linear table. The algorithm is very robust up to the Nyquist limit despite curved particle trajectories. Our relative displacement error is generally less than $5 \%$, and usually less than $2 \%$ for moderate ( $>1$ pixel) displacements.

\section{$2.5 \quad$ Derived quantities}

The location of the cross correlation peak gives the displacement of the center of the particle group in the interrogation window. The velocity of the particle group is simply the displacement divided by the time $\Delta t$ between exposures of the video images. $\Delta t$ is measured from the centers of the light pulses which illuminate each frame.

In two dimensions, the out of plane vorticity is given by

$$
\xi_{z}=\frac{\partial v}{\partial x}-\frac{\partial u}{\partial y}
$$

where $u$ and $v$ are the horizontal and vertical components of velocity respectively. We avoid direct differentiation of the velocity field by computing instead the circulation

$$
\Gamma=\oint \vec{u}(x, y) \cdot \overrightarrow{d \ell}
$$

where $\overrightarrow{d \ell}$ is a section of a closed contour of integration. In 2-D flow, Stokes' Law relates the circulation to the vorticity

$$
\xi_{z}=\frac{\Gamma}{\mathcal{A}}
$$

where $\mathcal{A}$ is the area enclosed by the integration contour. Hence, the vorticity is given in terms of circulation and area, which does not require differentiation of the velocity data. The circulation at node $i j$ is computed clockwise around a rectangular contour through the eight nearest neighbors 


$$
\begin{aligned}
\Gamma_{i j}= & \left(u_{i-1, j-1}+2 u_{i-1, j}+u_{i-1, j+1}\right) \frac{\delta x}{2} \\
& +\left(v_{i-1, j+1}+2 v_{i, j+1}+v_{i+1, j+1}\right) \frac{\delta y}{2} \\
& -\left(u_{i+1, j+1}+2 u_{i+1, j}+u_{i+1, j-1}\right) \frac{\delta x}{2} \\
& -\left(v_{i+1, j-1}+2 v_{i, j-1}+v_{i-1, j-1}\right) \frac{\delta y}{2}
\end{aligned}
$$

and the vorticity is

$$
\left(\xi_{z}\right)_{i j}=\frac{\Gamma_{i j}}{4 \delta x \delta y}
$$

Equation (2.8) compares well to direct differentiation of the velocity field and adds robustness to the vorticity calculation. Although the method spatially filters the field, the effect is not as severe as other methods which employ polynomial fits and a least-squares approach [135]. If only four data points are used in the calculation, that is, the integration contour encloses an area $\mathcal{A}=\delta x \delta y$, the method does not perform well. Similarly, if the spacing between data points is too small, for instance due to small interrogation windows and step sizes, high spatial frequencies are introduced into the result.

Although the circulation is given by equation (2.7), in some cases we would like to directly compute the circulation on a circular contour. For instance, to quantify the strength of a vortex, the circulation should be integrated along a circular path at a given radius from the center of the vortex. The method is a straightforward implementation of equation (2.5) using bilinear interpolation between data points.

The streamlines are obtained by computing the stream function

$$
\Psi=\int_{y} u d y-\int_{x} v d x
$$

The stream function is integrated twice, switching the order of integration over $x$ and $y$, and the two solutions are then averaged.

\subsection{Experimental apparatus}

Figure 2-2 is a schematic diagram of the experimental apparatus used throughout this thesis. A 6 Watt Argon-ion continuous wave laser generates a beam which is directed through a 


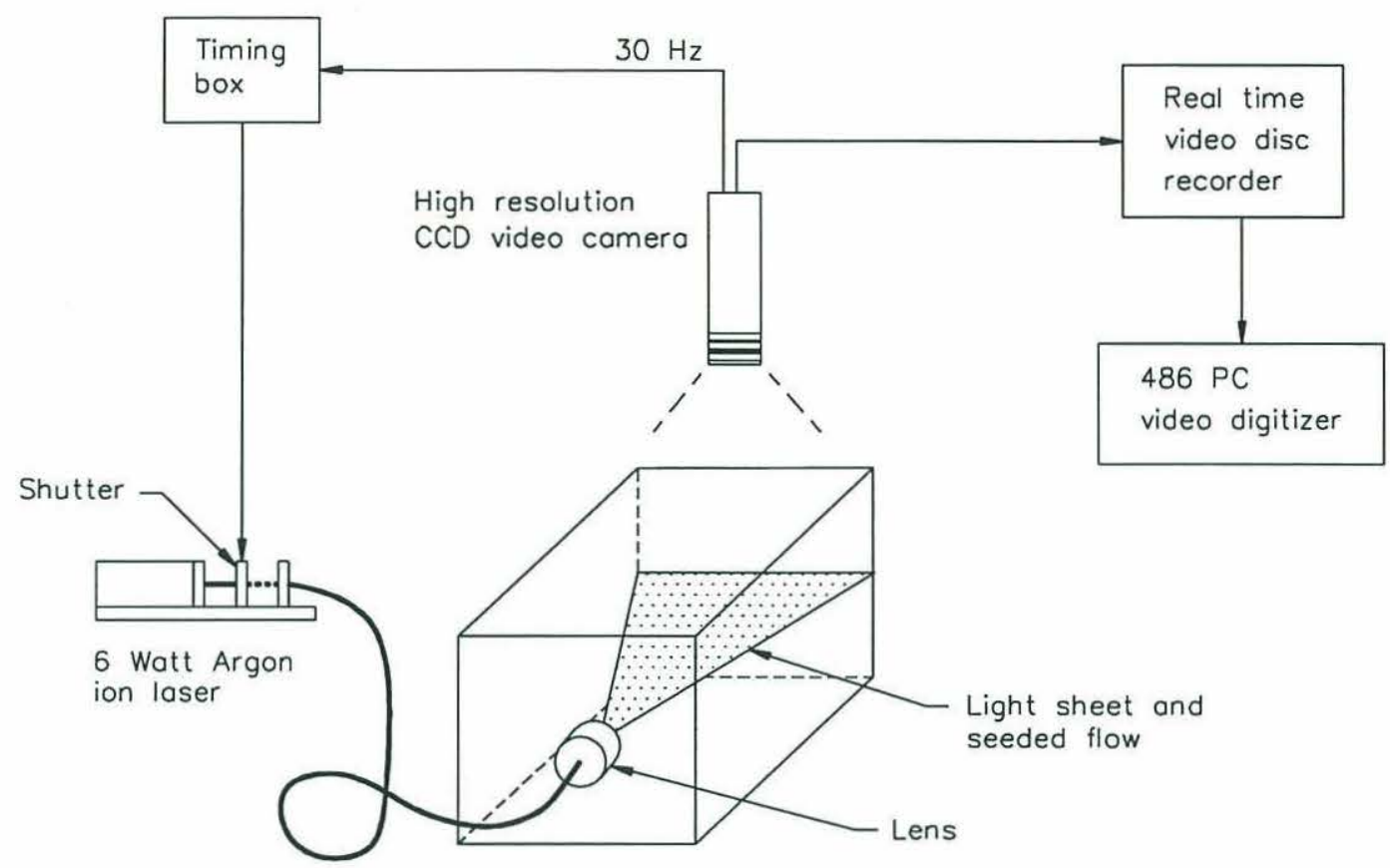

Figure 2-2: DPIV experimental apparatus

mechanical shutter and a fiber optic cable to the experimental area. The beam is passed through a lens to make a light sheet which illuminates a plane in the flow. The flow is seeded with small (diameter $\approx 40 \mu \mathrm{m}$ ) neutrally buoyant fluorescent particles and imaged with a high resolution, black and white, CCD video camera (Texas Instruments MULTICAM MC1134P). The camera has $1134 \times 480$ pixels at maximum resolution and acquires images at the standard video rate, $30 \mathrm{~Hz}$. The camera uses dual field exposure which allows full vertical resolution with no interlace.

The video signal is stored directly to a Sony CRVdisc videodisc recorder (LVR-5000A) in real time. A timing box (General Pixels) synchronized to the video signal controls the laser shutter. Although the camera frame rate is $30 \mathrm{~Hz}$, the effective framing rate can be increased by asynchronously shuttering the laser. Two successive light pulses are made to approach the video frame edge with a small time between them. Image pairs can be obtained with as little as a few milliseconds between them at half the camera rate, $15 \mathrm{~Hz}$. This allows us to study faster flows with acceptable pixel displacements.

Video frames of interest are then digitized to 8 bits/pixel using an EPIX 4MEG Video board in a 486 PC. The image pairs are correlated and filtered using software provided by 
Willert and Gharib [136] in addition to our own software.

The experiments documented in this thesis were all done in still fluid, i.e., water tanks instead of water tunnels. The DPIV method works equally well for either scenario. Still water is well suited for our experiments since we are usually concerned with shed vorticity that is relatively stationary. Hence, our desired frame of reference is fixed in the laboratory.

\subsection{Extensions of DPIV method near a moving body}

DPIV must be implemented carefully near any boundary such as a wall, free surface or moving body. Interrogation windows which cross a boundary contain image data which are not fluid. This biases the cross correlation peak towards the displacement of the boundary, resulting in spurious displacement data for the fluid near the boundary.

The DPIV algorithm is able to track some types of solid boundary without any special consideration. We expect this to be the case because the cross correlation function tracks patterns. However, if the body image is uniform, the correlation peak cannot be differentiated from the noise peaks and the data can be discarded on the basis of signal to noise ratio. If the boundary is a straight line, the cross correlation peak will track the displacement in the perpendicular direction, but cannot provide information on tangential displacement. If the object to be tracked is smaller than the interrogation window and has a distinct, nonuniform image, it can be located in both directions. The DPIV algorithm alone cannot locate deformable boundaries such as surface waves or undulating bodies.

Our goal in developing near-body DPIV techniques is to accurately track the flow near the boundary, not to track the boundary. Hence, we do not include any body or boundary data in our cross correlation calculations. It follows that we do not allow body or boundary data to enter into any of the post-processing such as filtering or vorticity calculations.

Figure 2-3 is a schematic diagram of the near-body DPIV algorithm. The standard DPIV algorithm generates raw velocity data. Before this data is interpreted, the data inside and near the boundary are deleted using an interactive software program. All data within half an interrogation window are discarded to ensure that none of the correlation calculations are contaminated by boundary data. Although it is possible to use image processing techniques to remove data inside boundaries automatically, we chose an interactive technique because our boundaries are typically deformable with highly variable characteristics. For instance, 


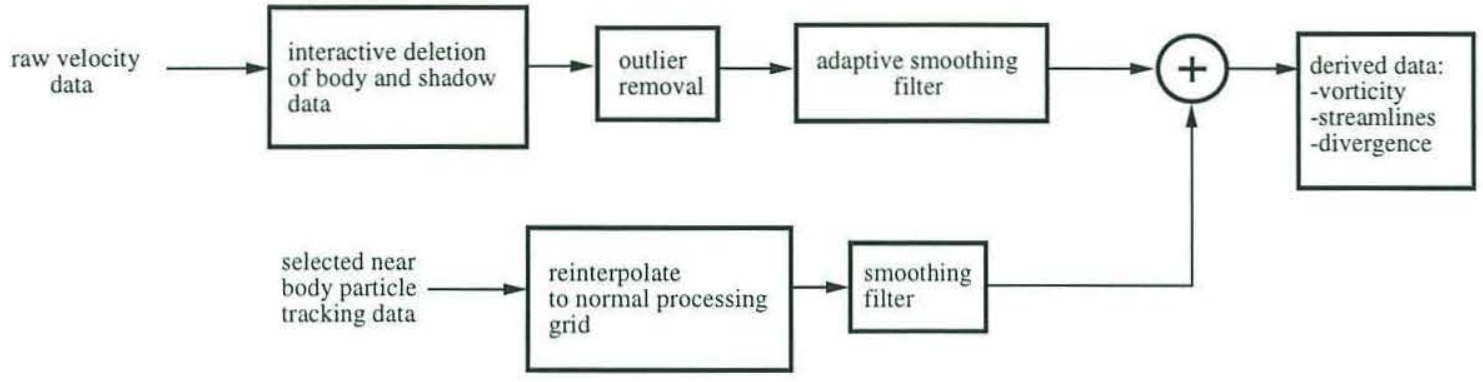

Figure 2-3: Schematic diagram of near-body DPIV algorithm. The standard technique is carefully applied in the region around the boundary and can be supplemented with particle tracking if required.

we often have a bright edge on the boundary which can cause reflections and shadow regions which are difficult to define because the edges are not always sharp. Sometimes particle correlations are possible along a shadow boundary. We leave these decisions to be made using interactive techniques based on the quality of the correlation peak.

Outlier removal and smoothing filters are then applied to remove the high frequency jitter inherent to DPIV. The algorithms are similar to the standard method except in that lacking data are treated specially. If a data point differs from at least four of its eight nearest neighbors by some threshold, the point is an outlier and is removed by linear interpolation. Horizontal velocities are interpolated with horizontal neighbors and vertical velocities are interpolated with vertical neighbors. If the necessary neighboring data point is missing, i.e., it is on or inside a boundary, it is not used in the interpolation. We then discard the outlier point so it does not contaminate any further derived data. Multiple passes are made through the outlier filter until no outliers remain.

The smoothing filter differs from the standard method by assigning differing weights to the neighbor points. As before, the filter averages the data point and its eight nearest neighbors. The data point itself has a weight of one, the diagonal neighbors have a weight of 0.8497 and the immediate neighbors have a weight of 0.7071 . These values follow a cosine function in both directions with the maximum at the center of the $3 \times 3$ grid. Weighting the filter in this way accommodates potential lack of data in the grid while minimizing possible bias in the result due to the remaining neighbors. If, say, a diagonal neighbor is missing, the data can still be smoothed and the diagonal data's weighted contribution removed from 
the calculation. If an immediate neighboring point is missing, its greater weight is removed from the average. If more than three neighbor points are missing, no smoothing is done but the point is not discarded. This filter removes jitter as well as possible without biasing the data when only a few data points are missing.

The adaptive nature of the smoothing filter was tested on a characteristic data set to see how well the filter handles missing data. Figure 2-4 summarizes the test case: a vortex pair measured behind an oscillating D-cylinder. The vortex pair flow is typical of our experiments as it contains high gradient regions and flow reversals.

Figure 2-4 (a) shows the vortex pair raw velocity data and artificially deleted data points. Three sets of three points were deleted in high, low and medium gradient areas. Figure 2-4 (b) shows the smoothed data, (c) the relative magnitude of the velocity gradient, and (d) error in smoothing caused by missing data. As expected, the magnitude of the error depends on the local velocity gradient. For this case, the maximum error is 15 percent of the maximum velocity in the field and occurs at the point of maximum velocity gradient. The filter performs well for medium and low gradient areas with a relative error less than five percent. This example demonstrates the behavior of the adaptive smoothing filter in the absence of data. It performs acceptably near absent data but must be used cautiously in high gradient regions.

When we want data within half an interrogation area from the boundary, we must resort to individual particle tracking. Individual particles can be tracked visually by closely examining each pixel value in the near-body region. This technique is tedious and accuracy suffers because the particle images generally occupy more than one pixel. Visual estimates give only whole pixel displacements whereas the cross correlation method gives subpixel accuracy. Our technique uses cross correlation with interrogation windows as small and as near the boundary as possible. Using this method, particle locations are accurately tracked with subpixel precision. The measured displacements are then converted to the same grid as the far flow field using bilinear interpolation.

Although this technique is numerical and partially automated, it still must be applied carefully near the boundary. Images near the boundary may be shadowed or contain reflections from one video frame to the next. The very near-body flow is difficult but not impossible to obtain. Because of this, we only use this technique when we are specifically interested in the very near body region. For the general case, we apply only the top flow 

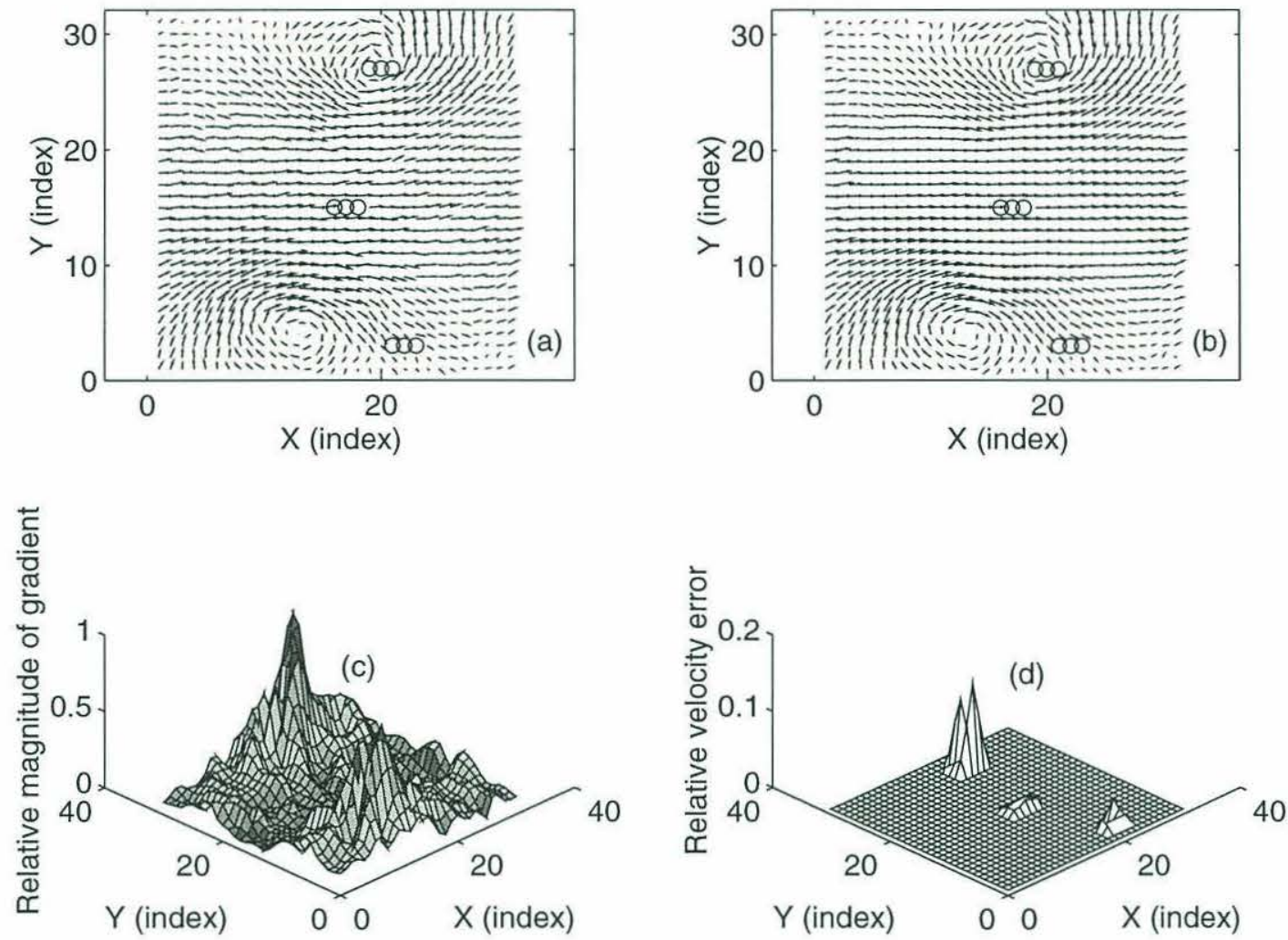

Figure 2-4: Test case for adaptive smoothing filter. (a) raw data with artificially removed data indicated by circles, (b) smoothed data, (c) relative magnitude of velocity gradient, (d) relative velocity error compared to maximum velocity. 


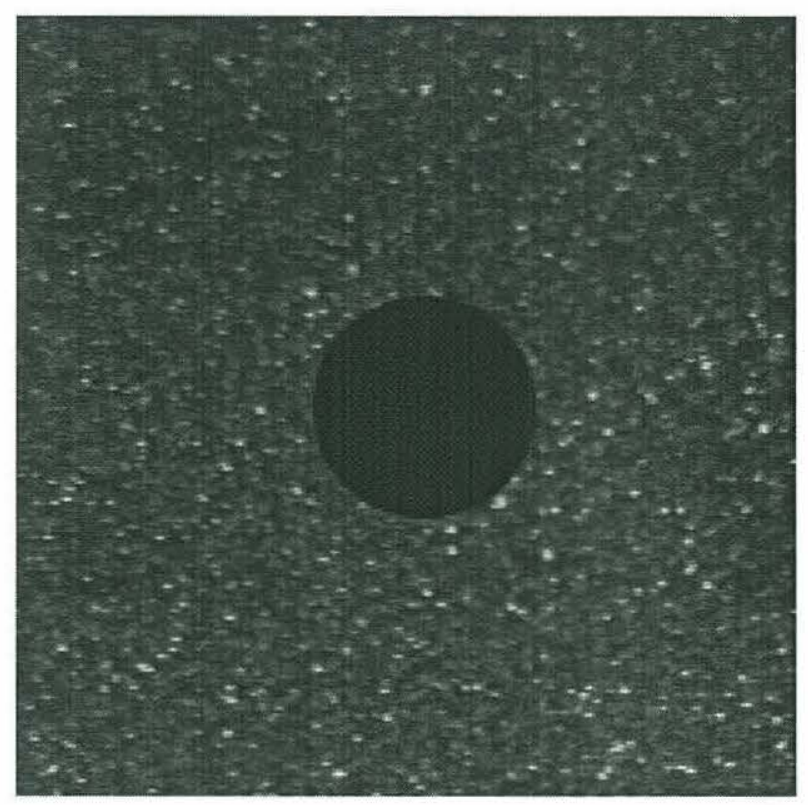

Figure 2-5: Artificial cylinder image used to verify near-body DPIV method

path illustrated in Figure 2-3.

Our standard method (the upper path in Figure 2-3) was verified with two contrived near-body scenarios. A cylindrical body was artificially created in a real particle image. In Case A, we shifted the image data to the right by five pixels while keeping the cylinder stationary. Case B is the opposite scenario: the cylinder is moved five pixels to the right while the image data are fixed. Figure 2-5 shows the test image and Figure 2-6 shows the raw and processed data for the two cases. The data were processed with $16 \times 16$ pixel interrogation windows, every four pixels. The cylinder is 64 pixels (four interrogation windows) in diameter.

We chose to process this data with a $16 \times 16$ window to challenge the algorithm. The pixel shift of 5 pixels is near the maximum allowable displacement of one third the window dimension. A small window size is always preferable because it allows us to get data closer to the boundary. Unfortunately, the seeding density ultimately limits our window size. Figure 2-6 (a) clearly shows the jitter associated with miscorrelations due to moderately low seeding for this window size. Spurious data near the boundary occur in both scenarios (Figure 2-6 (a) and (b)). Both cases effectively demonstrate the necessity of special treatment near boundaries and they validate our methods. Close examination of the filtered data shows no contamination of the result by boundary data. These cases confirm the near 
(a)

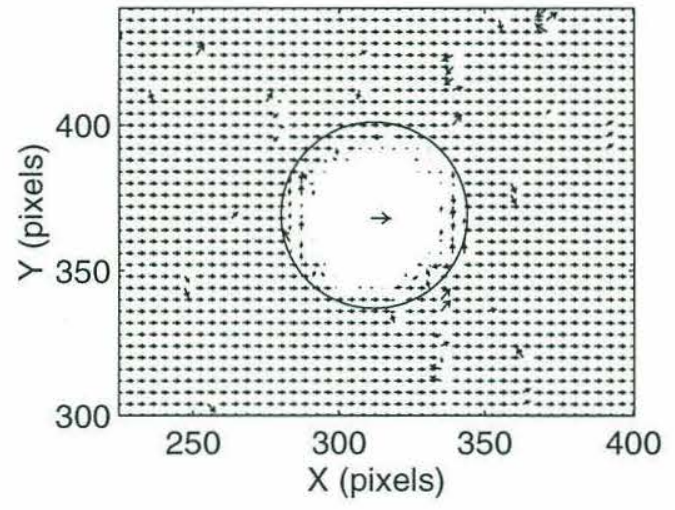

(c)

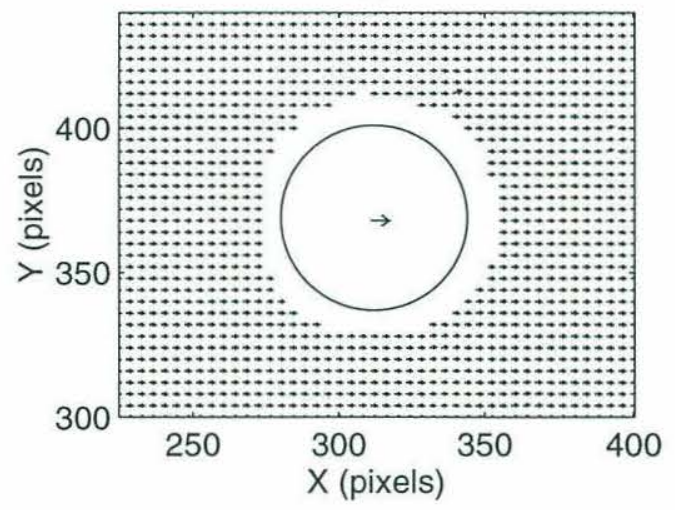

(b)

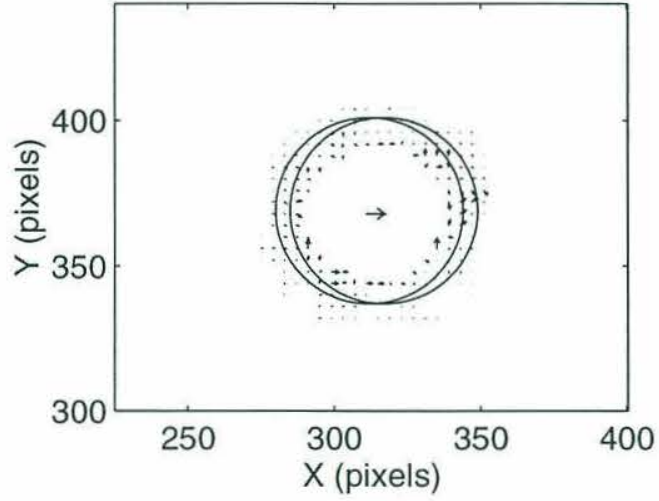

(d)

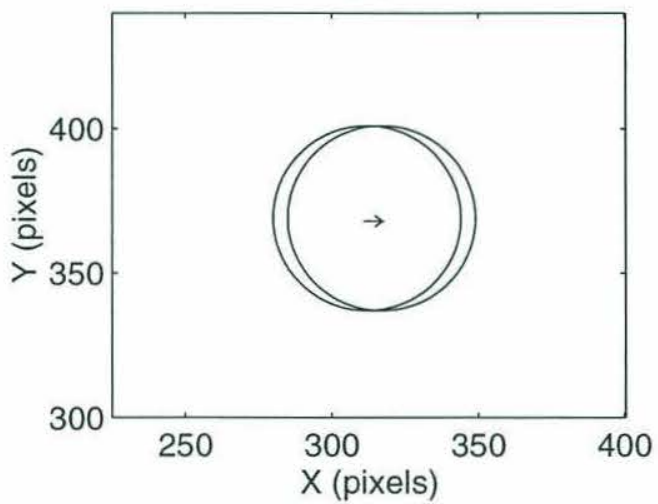

Figure 2-6: Two artificial near body test cases. Case A: cylinder held fixed while particle images shifted by 5 pixels to the right. Case B: particle images held fixed while cylinder image shifted 5 pixels to the right. (a) Case A raw data; note near-body false correlations (b) Case B raw data, (c) Case A near-body processed data, (d) Case B near-body processed data. An artificial vector is shown in the center of the cylinder to guarantee the same scale for the four plots. 
body data deletion technique for both fixed and moving bodies. Data very near the body can be obtained using the lower path algorithm in Figure 2-3 if necessary. In this thesis, we implement only data deletion and adaptive filtering near boundaries as particle tracking in the near body region is computationally expensive.

\subsection{Summary}

In this chapter, we have summarized the method of digital particle velocimetry as implemented in this thesis. Digital particle image velocimetry provides nonintrusive multipoint quantitative flow measurements acquired in real time. As DPIV operates quickly using digital means, we are now faced with the burden of interpretation of the data rather than by the difficulties associated with the experimental system. In this thesis, we will apply DPIV to a variety of unsteady problems which would be cumbersome to study using traditional single point measurement methods. 


\section{Chapter 3}

\section{Experimental study of fish propulsion and maneuvering}

"With motion irrotational, in fluid incompressible, A tiny little minnow swims along a line of flow, And the greater its velocity-well cutting out verbosityThe greater its velocity, the faster it will go."1

\subsection{Introduction}

The subject of fish swimming has been studied for many years by biologists as well many distinguished fluid mechanicists such as Gray [42], Lighthill [64], Wu [141] and others. Fish are able to propel themselves for great distances and accelerate and maneuver with intriguing agility.

In this chapter we present experimental results for the flow around a live fish while swimming straight and turning. Flow visualization and digital particle image velocimetry (DPIV) are used to study their propulsive capabilities with emphasis on vortex generation and placement in the wake. Although some researchers have attempted visualizations of swimming fish, our quantitative measurement of the velocity field around a live swimming fish is the first of its type.

\footnotetext{
${ }^{1}$ Eureka, Cambridge from [75]
} 


\subsection{Background}

The earliest critical observations of fish swimming are usually attributed to Breder [18] who classified fish swimming according to the extent of the body that is included in locomotion. Anguilliform swimming named after the eel Anguilla, utilizes large amplitude motions over a long thin flexible tail which comprises a large fraction of the body. Carangiform swimmers possess tapering tails of medium length which terminate in a well defined caudal fin. The primary propulsive movements are limited to the tail region. Ostraciiform fishes have relatively stiff bodies and oscillate only their caudal fins at the point of attachment as if it were a hinge. Each of the major classes of swimming contain several sub-groups which further depend on the flexibility of the tail and the extent of the body involved in the propulsive movements. Notably, Thunniform swimming, named after tunas and related fishes which swim similarly, is an example of carangiform swimming where the anterior portion of the body is very stiff and all propulsive movements are confined to a small region near the high aspect ratio lunate tail.

Much of the early work on fish swimming hydrodynamics concentrated on the notion of drag reduction due to undulatory motion and devices which affect the boundary layer such as slime and dermal denticles as in the case of sharks. In 1936, Gray [41] spawned much interest in the subject by presenting Gray's Paradox which concludes that porpoises and dolphins require seven times more power than they have available in their propulsive musculature to achieve their swimming speeds. Gray's studies assume simple hydrodynamic resistance and muscle power models of human muscle to reach the conclusion that porpoises and dolphins either have more muscle power available or that they are able to maintain a laminar boundary layer. Gray's Paradox has been the subject of much debate for decades and we do not attempt to make any contributions to the arguments in this thesis.

More recently, $\mathrm{Wu}[141,142,143,144]$ and Lighthill $[62,63,64]$ have developed reactive theories that emphasize inertial effects of the undulatory body and are more appropriate than simple resistive models for the high Reynolds number swimming of typical fish. In two dimensions, $\mathrm{Wu}$ [142] derived the flow around a flexible plate, swimming at arbitrary unsteady forward speeds using linearized inviscid flow theory. Lighthill [62] developed a three-dimensional elongated body theory in which each section along the body of the fish is considered individually and the effects of local virtual mass are integrated over the length 

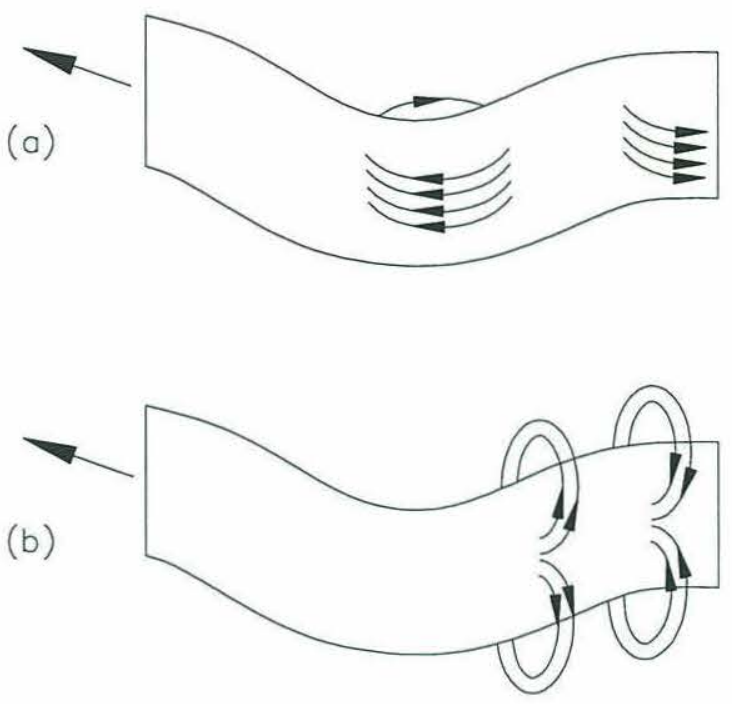

Figure 3-1: Flow along a swimming fish: (a) two-dimensional swimming theory, (b) threedimensional elongated body theory.

of the body.

In Wu's 2-D model, the fluid near the body moves longitudinally (parallel to the direction of swimming) with the passage of a travelling wave along the body as shown in Figure 31(a). In Figure 3-1, the body moves to the left as the travelling wave moves to the right. In contrast, Lighthill's 3-D model neglects the tangential motion of fluid particles along the body. The fluid particles are assumed to move primarily in transverse planes at each section along the body as illustrated in Figure 3-1(b). Weihs [131] extended Lighthill's inviscid elongated body theory to include the lift forces (due to circulation) from the fins and tail using unsteady aerodynamics.

Recently, Triantafyllou et al. [118] surveyed the biological literature and reported that most fish and marine mammals swim in the same manner characterized by the Strouhal number, defined as

$$
S t=\frac{f A}{U}
$$

where $f$ is the frequency of tail oscillation in Hertz, $A$ is the double amplitude tail excursion and $U$ is the forward velocity. Triantafyllou et al. found that most fast-swimming fish oscillate their tails with $0.25<S t<0.35$, regardless of size. These results support earlier work by Triantafyllou et al. [120] which used a stability analysis of the jet wake of an 
oscillating foil to show that the wake is convectively unstable for $0.25<S t<0.40$. The wake is a tuned amplifier with the frequency of maximum spatial growth of the disturbances (and optimum propulsive efficiency) at $S t \approx 0.3$. The resulting wake formation is a staggered array of vortices in the opposite direction of the classic Kármán street. Fish may exploit this phenomenon to generate maximum thrust with minimum input work.

A current topic of interest in fish hydrodynamics is numerical simulation of fish swimming using vortex methods and viscous codes. A interesting recent computational work by Nakaoka and Toda [78] studied a two-dimensional wing which moves like a fish using an unsteady viscous code at Reynolds number $1 \times 10^{4}$. A NACA 0010 wing was used to approximate the planform of the fish body whose sections were displaced laterally in a travelling wave from nose to tail to simulate swimming movements. Three simplified body motions were taken from rainbow trout experiments. The body was placed in a uniform flow and oscillated with peak to peak tail sweep of 0.20 body lengths and frequency 1.6 to $2.0 \mathrm{~Hz}$. Because the prescribed motions are taken from actual fish kinematic data, the Strouhal numbers range between 0.32 and 0.40 . Nakaoka and Toda's simulations produced net thrust of the order of the drag force on a stationary foil. The jet wake structure is a staggered alternating array of vortices arranged near the centerline. The simulation which produced the most thrust produced an array that was 0.1 body lengths wide, half of the tail double amplitude. Future simulations of this type look promising.

Several excellent reviews on the subject of fish swimming have been published which summarize the traditional swimming theories and address a myriad of specific morphological adaptations. Gray [42], Lighthill [64], Blake [10], Videler [123], Yates [146] and Magnuson [66] are but a few of the many authors who have prepared detailed compilations of the current understanding of fish swimming.

In this chapter, our goal is not to dispel traditional swimming theories, but rather to clarify the flow structure alongside and in the wake of a swimming fish. Flow visualization with Kalliroscopic fluid and quantitative measurements with DPIV are the first measurements of this type which quantify the details of the propulsion mechanisms used by a live, naturally swimming fish. In James Gray's own words, “... there is not the slightest doubt that a most valuable picture of fish propulsion would emerge if it were possible to relate the speed of the animal to the precise changes in shape exhibited by the body, and to the patterns of disturbance which these changes induce in the surrounding water." [42]. 


\subsection{Flow visualization of swimming fish}

Our motivation to visualize a swimming fish is largely due to work by M.W. Rosen [96]. Rosen visualized the flow around a small tropical fish, the Pearl Danio Brachydanio albolineatus, and made some controversial observations. Rosen visualized the fish in a narrow, shallow raceway which was $3.8 \mathrm{~cm}$ wide and $2.54 \mathrm{~cm}$ deep with a $1.6 \mathrm{~mm}$ milk layer on the bottom of the tank which revealed a black background when disturbed. Vortex-like structures were observed along the body immediately behind the gills in a Kármán-like drag orientation, which are then convected downstream and repositioned by the tail in a single line in the wake of the fish.

Figure 3-2 shows one of Rosen's photographic sequences in which the fish swam upwards from the bottom of each frame. Figure 3-2 shows what appears to be a free vortex to the right side of the fish which moves posteriorly, eventually to interact with the tail. An alternating array of vortices persist in the wake, in-line with the direction of the fish. Because the vortices in the wake lie on a single line, the fish produces a wake profile that is neither drag nor thrust. Rosen postulated that the fish creates drag vortices, which it then uses like pegs, to push off to propel forward. Energy spent in creating drag vortices is recaptured by the tail and aids in generating a thrust vortex street. Our first goal was to verify this result using more quantitative techniques.

In the spirit of Rosen's work, we chose to experiment with a similar fast swimming tropical species, Danio malabaricus, the Giant Danio. Figure 3-3 shows the morphology of the Giant Danio which is very similar to that of the Pearl Danio. The dominant fins are the flexible dorsal fin and the stiffer anal fin which acts like a keel. Anterior to the anal fin, the ventral fins flare out during maneuvering but participate little in straight line swimming. Similarly, the pectoral fins are used in maneuvering and are generally kept flush to the body in straight line motion. The region of narrow necking immediately anterior to the caudal fin is referred to as the caudal peduncle.

Several fish were used with varying lengths between 5 and $10 \mathrm{~cm}$. The maximum span of the caudal fin was approximately 23 percent of the body length. Whenever possible, data will be presented normalized with respect to fish body length, hereafter denoted as $\ell$. We found the Danios well suited to our experiments since they are steady, fast swimmers and hardy with respect to handling. 


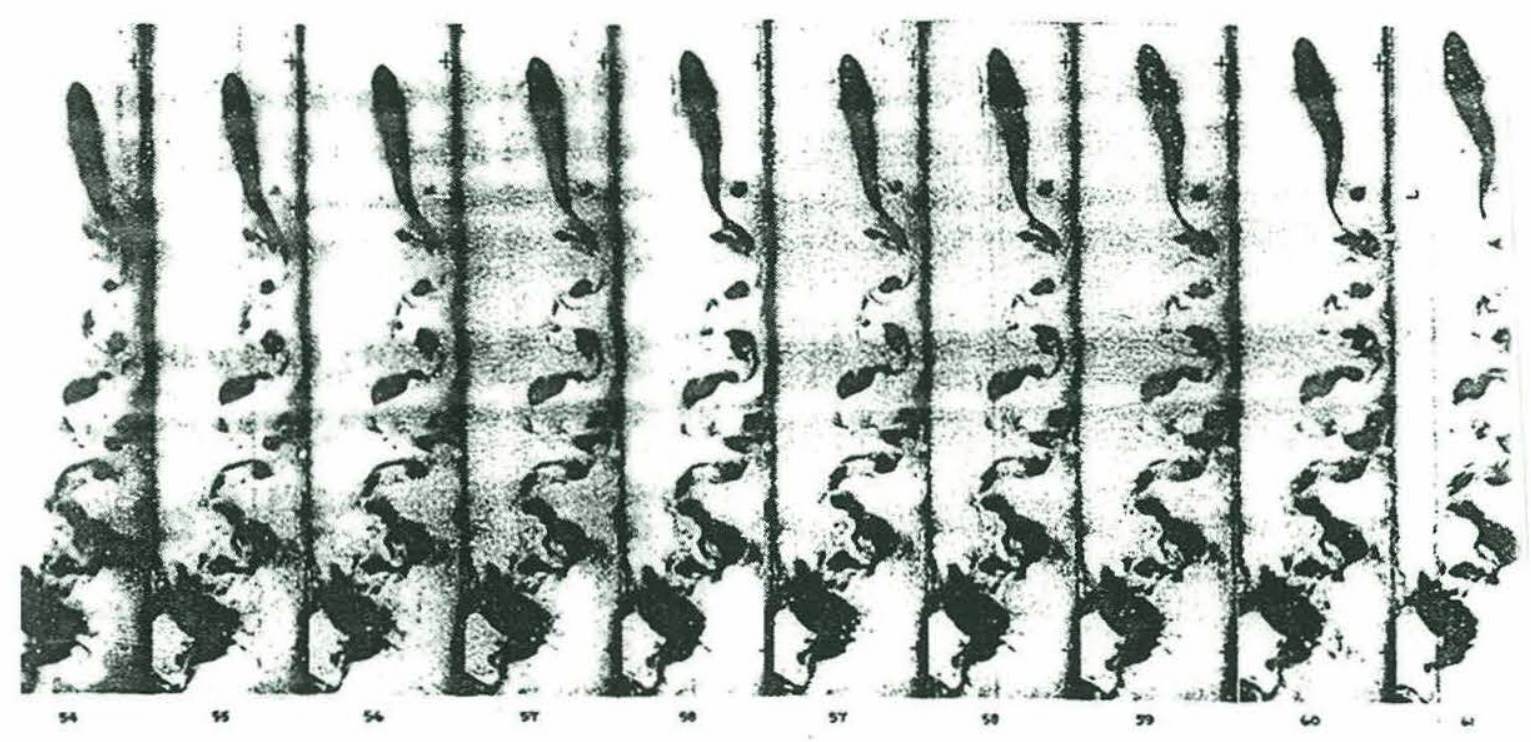

Figure 3-2: Photographic sequence of Pearl Danio swimming visualized with milk layer against a black background, adapted from Rosen (1959), Figure 13, Run II. Approximately half a tailbeat cycle is shown, in 0.01 second increments

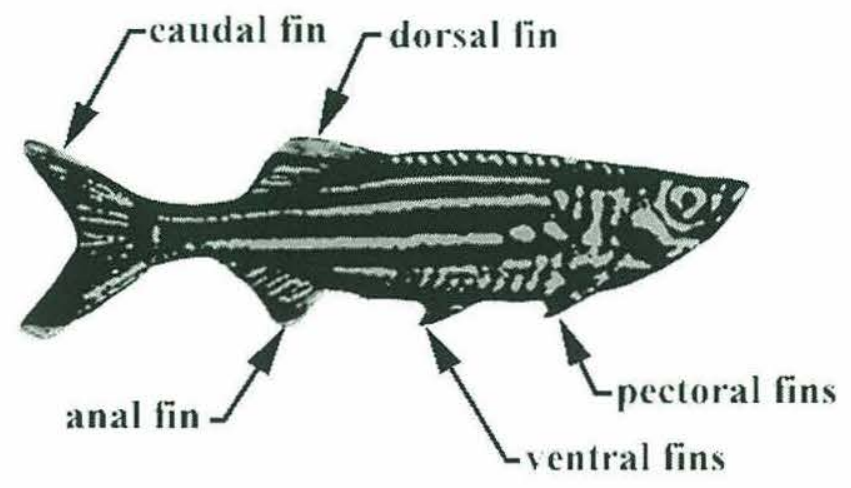

Figure 3-3: Giant Danio morphology 
Our first experiments were in a shallow tank, similar to Rosen's tank but wider. Rosen's tank was roughly one fish body length in width whereas ours is approximately two body lengths $(15 \mathrm{~cm})$ wide. The first issue to resolve with respect to Rosen's results was whether the side walls of his tank compressed the observed wake. The wall effect is significant as walls increase the stability of the wake while compressing the vortex street [116].

Instead of duplicating Rosen's milk layer technique, we chose to use a weak solution of Kalliroscopic fluid and water. Kalliroscopic fluid (K-fluid) is a commercially available product consisting of organically derived guanine flakes. The flakes are highly reflective, anisotropic and very small, $6 \times 30 \times 0.07 \mu \mathrm{m}$. A blue aqueous dye added to the solution improves contrast. The K-fluid solution is opaque which allows visualizations only at the free surface.

K-fluid visualizations have been used by a number of experimenters $[40,67]$ and have been compared favorably with laser doppler velocimetry. Savaş [104] showed using stochastic methods that the particles align with the stream surfaces in the flow, producing a color change against the still background which can be imaged. The technique is suitable only for large scale flows with large shear perturbations. The method is appropriate for our experiments since they involve large scale vortical structures against a still background.

The opacity of the K-fluid limited the water depth to $2.54 \mathrm{~cm}$ so that the fish body could be seen. The $\mathrm{pH}$ was adjusted to neutral and the temperature ranged between $24^{\circ}$ and $29^{\circ} \mathrm{C}$. Bright flood lights were used to heat and smooth the free surface of any visible convection cells. The fish was confined in a holding area at one end of the tank until the imaging area was settled. A gate was removed and the fish was free to swim across the camera field of view through another gate into another holding area. The fish responded well to the K-fluid solution, but were agitated in shallow depths.

The flow patterns were imaged with a Kodak EKTAPRO Hi-Spec Motion Analyzer on loan from the Edgerton Center at the Massachusetts Institute of Technology. Images were acquired at 1000 frames per second into a cyclic buffer with 2400 frames of storage. The resulting images had resolution of $192 \times 239$ pixels with 256 levels of gray. The video was then replayed at slower rates to a VHS recorder. The VHS tape was then digitized to 768 x 480 pixels using an EPIX 4MEG-video board in a $486 \mathrm{PC}$. The images were interrogated manually with a cursor to measure flow features.

Figure 3-4 shows two separate video frames, roughly one quarter of the tailbeat cycle 


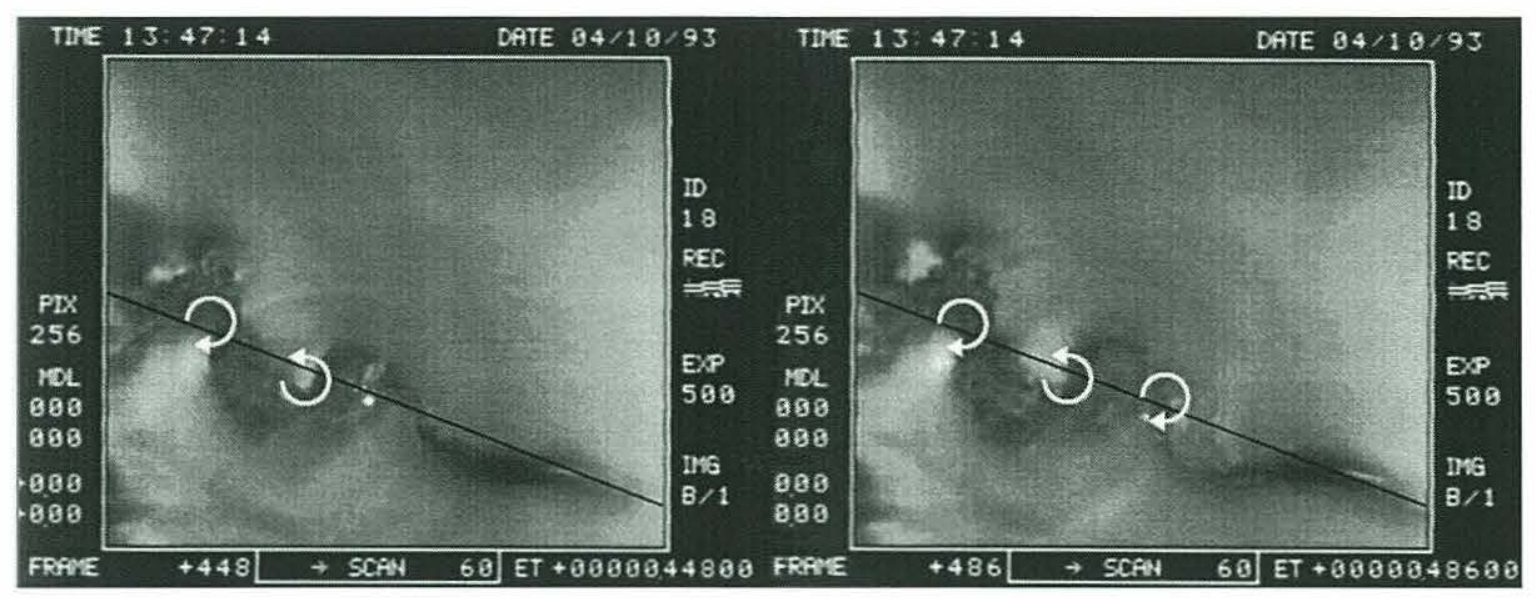

Figure 3-4: K-fluid visualization of straight swimming fish. Frames are approximately $\frac{1}{4}$ cycle (0.038 seconds) apart. Fish direction is shown with a black line, vortices with white arrows. The fish nose is near the lower right hand corner in each frame.

apart. The still photographs show the sinuous trajectory of the tail clearly because of the strong shear layer. Less apparent in the still photographs is the array of alternating vortices near the centerline of the fish motion, indicated with white arrows. The wake width, i.e., the lateral distance between vortex centers is roughly 15 percent of the tail double amplitude or 5 percent of the fish body length. In this sequence, the fish is moving at $5.5 \frac{\ell}{s}$, the tailbeat frequency is $7.1 \mathrm{~Hz}$ and the double amplitude of the tail motion is $0.25 \ell$, averaged over one and a half cycles. The nominal Strouhal number for this motion is 0.33 which is in the optimal range predicted by Triantafyllou et al. [118].

The downstream wake agrees qualitatively with the results of Rosen. There is an alternating array of vortices, producing a weak jet in our case instead of neutral wake. However, no free vorticity along the body was observed. The experiment was repeated several times in an attempt to visualize near body vorticity. None was found, despite very shallow fluid depths. This leaves us to conclude that free vorticity does not exist near the body or that the existing vorticity is so weak that $\mathrm{K}$-fluid cannot resolve the feature.

In addition to the milk layer experiments, Rosen performed a dye wake visualization by dipping the fish in blue dye and dropping it quickly into the tank. A wake pattern was left when the fish swam through clean water. There was no indication of a separated vortex near the body as in the milk layer experiments. This confirms that the phenomenon observed in the milk experiments is not free vorticity as initially concluded. If a drag vortex sheds from the body, it would carry dye with it and appear in the dye visualization. We will discuss 
this phenomenon further in the following section.

Direct comparisons between our result and Rosen's must be made cautiously. Rosen's

Danio swam much faster than ours, $14.8 \frac{\ell}{s}$ compared to our observed $5.5 \frac{\ell}{s}$. The average Strouhal number for Rosen's runs was 0.74 , which differs from the optimal value significantly. In addition, we cannot rule out the fact that a fish may not always swim optimally, especially in times of stress. Ideally, we would like the fish to swim as naturally as possible.

\subsection{Shallow water DPIV experiments on straight swimming fish}

The success of the K-fluid visualizations encouraged us to proceed to DPIV to quantitatively measure the flow around a swimming fish. The first set of experiments were done in shallow water $(2.54 \mathrm{~cm})$ in the same tank as the $\mathrm{K}$-fluid visualizations. The laser light sheet was oriented horizontally in the midplane so that it hit the fish near the vertical plane of symmetry. The laser light sheet was approximately $3 \mathrm{~mm}$ thick. The water was reverse osmosis filtered, adjusted to neutral $\mathrm{pH}$ and $24^{\circ} \mathrm{C}$ and seeded with $40-60 \mu \mathrm{m}$ diameter, neutrally buoyant, fluorescent polymer spheres. The fish showed no adverse effects to the particles or laser light.

The flow around the fish was imaged from above through the free surface with a Texas Instruments MULTICAM MC-1134P high resolution black and white CCD video camera. As before, the fish was confined to one end of the tank until the tank was settled, then allowed to swim into the camera field of view. In all of the runs the tank width was $15 \mathrm{~cm}$, although the camera view varies. Blockage effects due to the side walls are expected to be insignificant as the tank width is much greater that the fish body width.

Figure 3-5 shows the digitized trajectory of the fish for the shallow experiment. The fish travelled in a straight line away from the tank wall located at $\mathrm{Y}=15 \mathrm{~cm}$. Figures $3-6$ through 3-17 show the velocity and vorticity results for this case for approximately one cycle of the body motion. In these figures, the laser light sheet illuminated the fish from above which cast a shadow on the lower side of the fish. Due to the extremely shallow water, the velocity field has high divergence and many outliers. The velocity data are filtered using the methods described in Chapter 2 with minimal success. Persistent outliers due to the strong three dimensional flow could not be removed with the standard post processing techniques. 


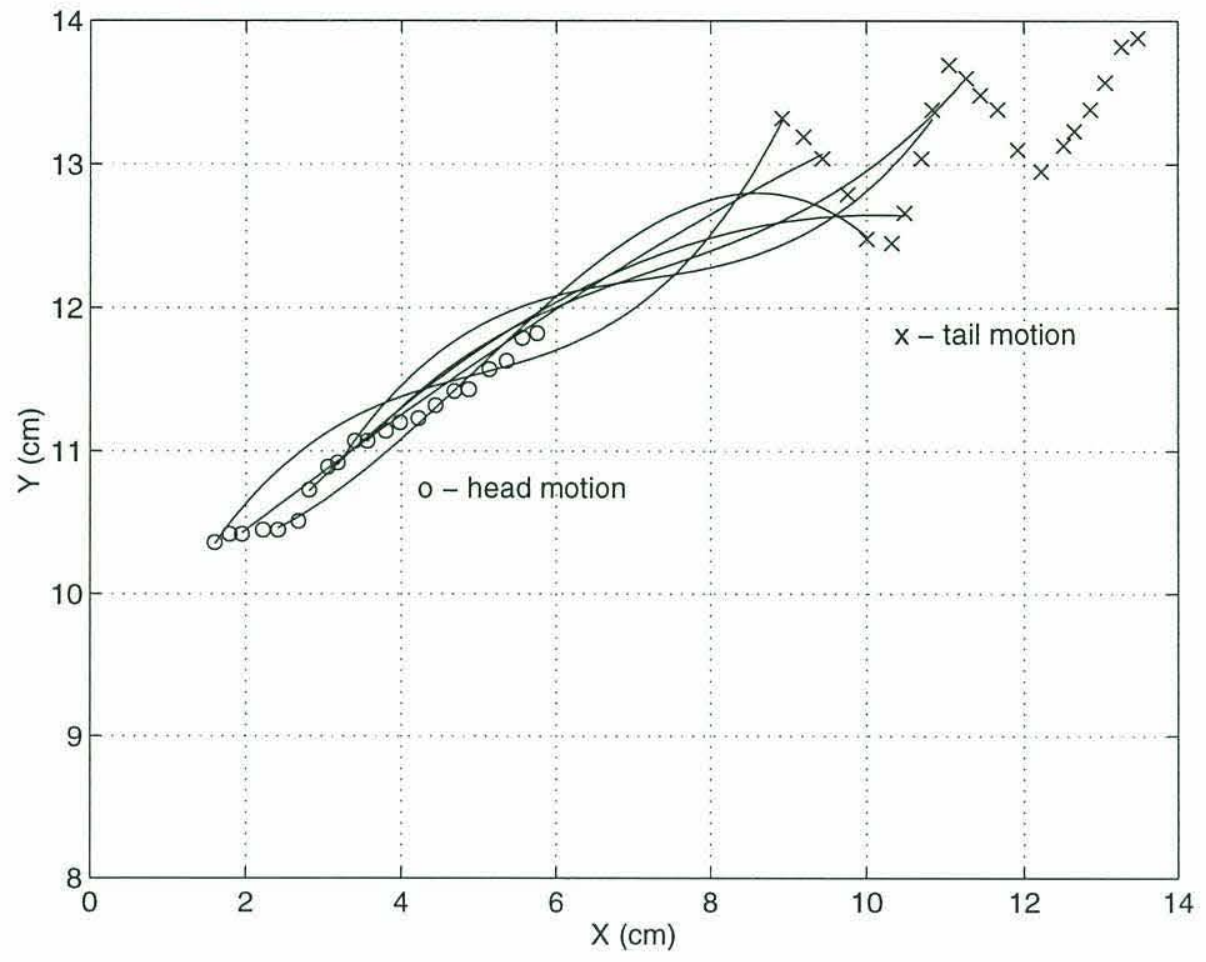

Figure 3-5: Trajectory of straight swimming fish. The ' $\mathrm{o}$ ' and ' $\mathrm{x}$ ' marks indicate head and tail motions respectively. The flow around the fish is presented in the following figures for the body positions shown with a curved line, roughly one cycle. The vertical scale is enlarged to show the tail path more clearly.

The wake flow features are better revealed in the vorticity contours since by the nature of the calculation, the high spatial frequencies in the data are attenuated. The fish body image is overlaid in the following figures exactly as it appears in the first frame of each image pair.

In Figures 3-6 and 3-7, the fish is shown beginning the cycle with the tail near the maximum upward excursion. Data near the body and in the shadow of the body have been removed as described in Chapter 2. Prior to this instant, the fish swam from right to left, upwards towards the wall. The wake from previous swimming cycles persists along this trajectory. The velocity data show a weak jet and concentrated regions of vorticity which are well defined in the vorticity contours shown in Figure 3-7. Dark contours indicate negative (clockwise) vorticity whereas light contours indicate positive (counterclockwise) vorticity. The wake vorticity is arranged in a staggered array of counter-rotating vortices whose combined effect produces a net jet along the centerline of the array. 


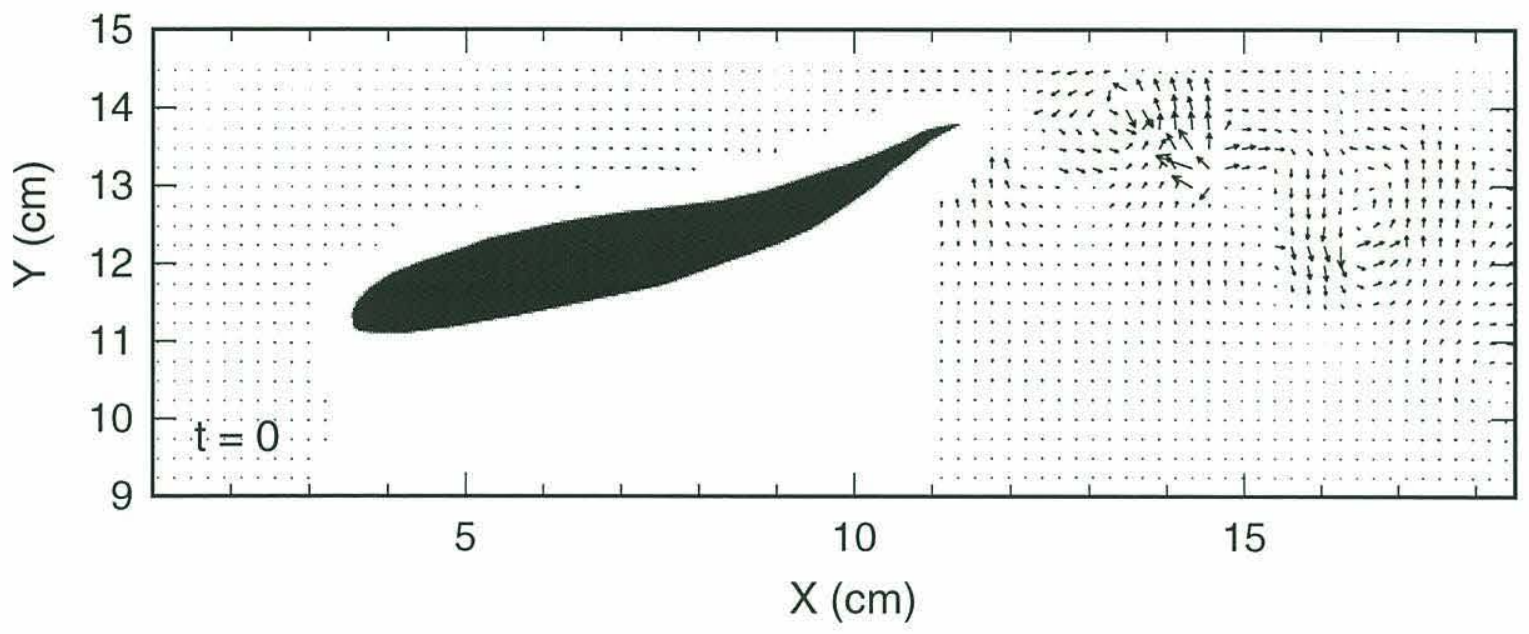

Figure 3-6: Velocity field in the wake of a swimming fish in shallow water. The tail is near the position of maximum vertical excursion.

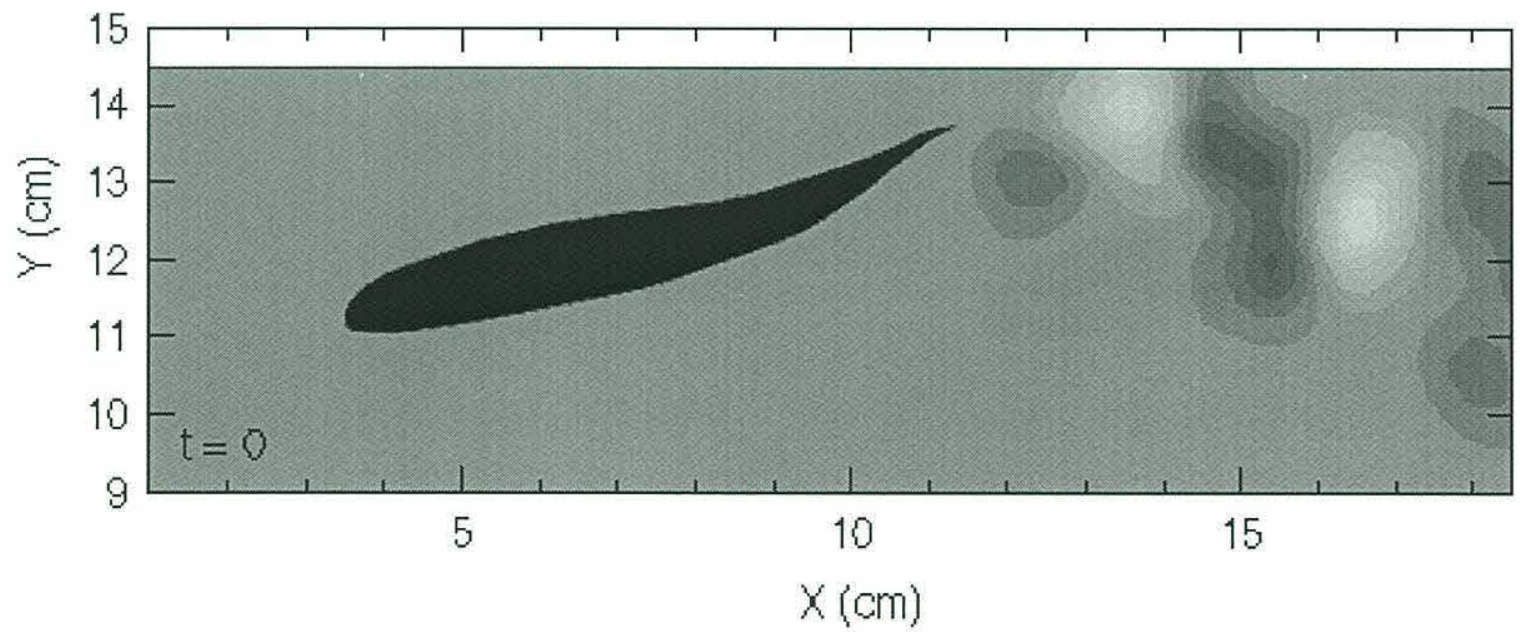

Figure 3-7: Vorticity field in the wake of a swimming fish in shallow water. Contours are from -10.0 (black) to $10.0 \mathrm{~s}^{-1}$ (white) in $1.0 \mathrm{~s}^{-1}$ increments. 
In Figures 3-8 and 3-9, the tail begins to move downward forming a positive vortex in the vicinity of the tail. The flow is consistent with that of a rigid foil moving downwards which generates counterclockwise (positive) vorticity at the trailing edge. The vorticity contours however, do not show this newborn vorticity yet because it is too near the body to be realized accurately. This vorticity first appears as a distinct vortex in Figure 3-11 once it has convected sufficiently away from the near body region to be resolved by our method.

Figure 3-12 and 3-13 show the tail at its maximum downward position poised to move upwards. Similar to the downstroke, oppositely signed vorticity is generated at the trailing edge, and as before does not appear in the vorticity contours until Figure 3-15 when the vorticity is farther away from the body. In Figure 3-16 and 3-17, the swimming cycle is back at the starting position.

\subsubsection{Discussion}

The shallow water experiment confirms the fundamental aspects of straight line fish swimming. In each swimming cycle, two vortices are formed which combine in the wake to form a net jet. Careful examination of the raw video data near the body (not shown in the presented results) indicate that no free vorticity forms along the body which confirms our earlier result.

However, the undulating body does contribute to longitudinal (streamwise) variations in the outer flow. Figures 3-14 and 3-16 clearly show velocity vectors pointed both upstream and downstream which converge in a region pointed towards the fish mid-body. Figure 318 schematically summarizes the flow near the body and vortex generation in the wake. The near-body flow is related to the movement of a travelling wave along the fish. This is contrary to Lighthill's elongated body theory [64] which assumes that at a given crosssection, fluid particles in the vicinity of the body move primarily laterally (towards or away from the fish surface in a normal direction) or vertically. Here, we see significant longitudinal motion induced by bound vorticity which follows the crests and troughs of the travelling wave.

Several authors have completed detailed cinematographic studies of the kinematics of fish swimming (Videler and Hess [122] for example) and have observed that in carangiform and thunniform swimming modes, a travelling wave of increasing amplitude moves along the body from the head towards the tail, displacing each longitudinal section. Often, this 


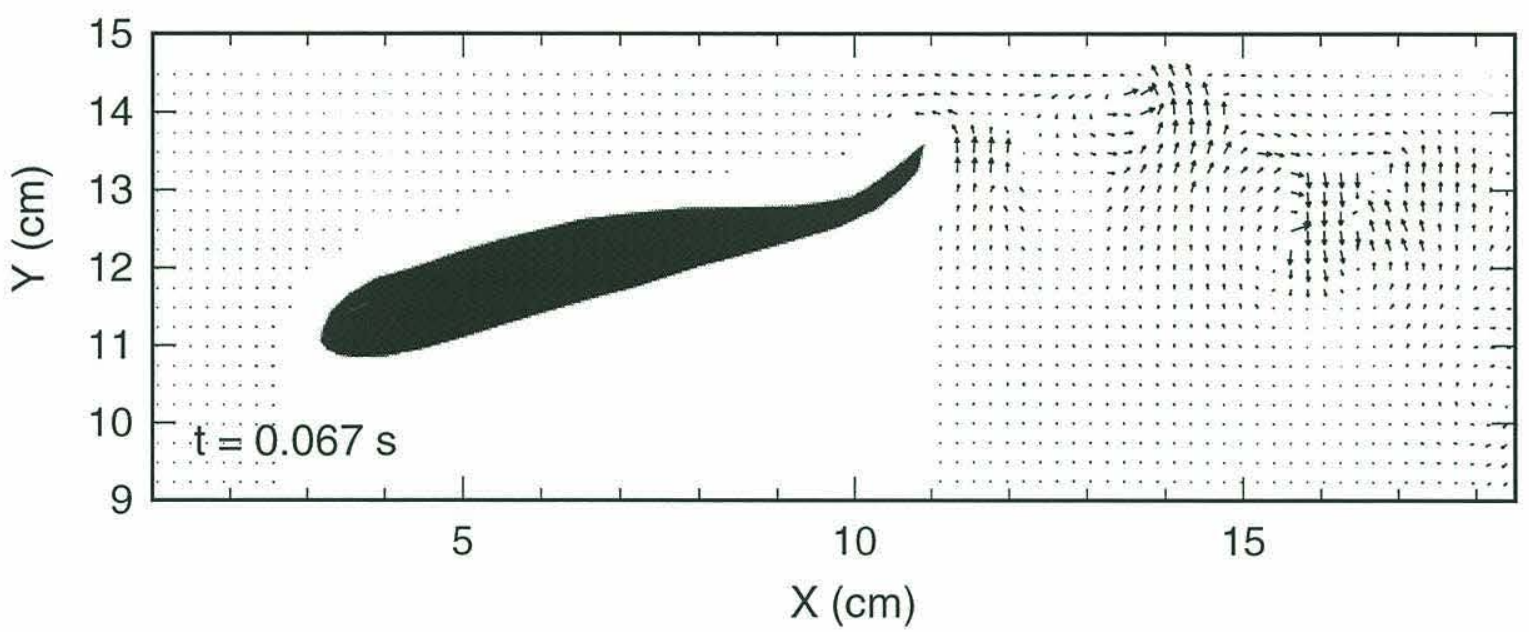

Figure 3-8: Velocity field in the wake of a swimming fish in shallow water.

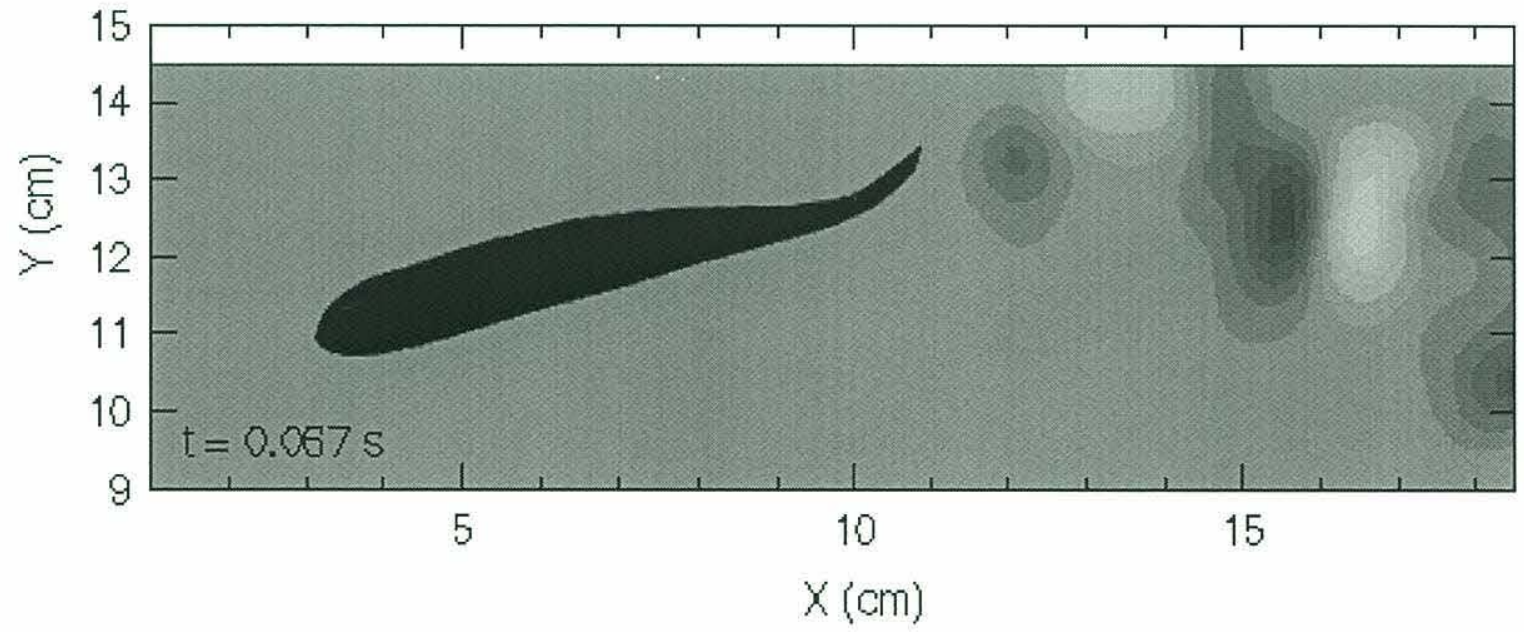

Figure 3-9: Vorticity field in the wake of a swimming fish in shallow water. Contours are from -10.0 to $10.0 \mathrm{~s}^{-1}$ in $1.0 \mathrm{~s}^{-1}$ increments. 


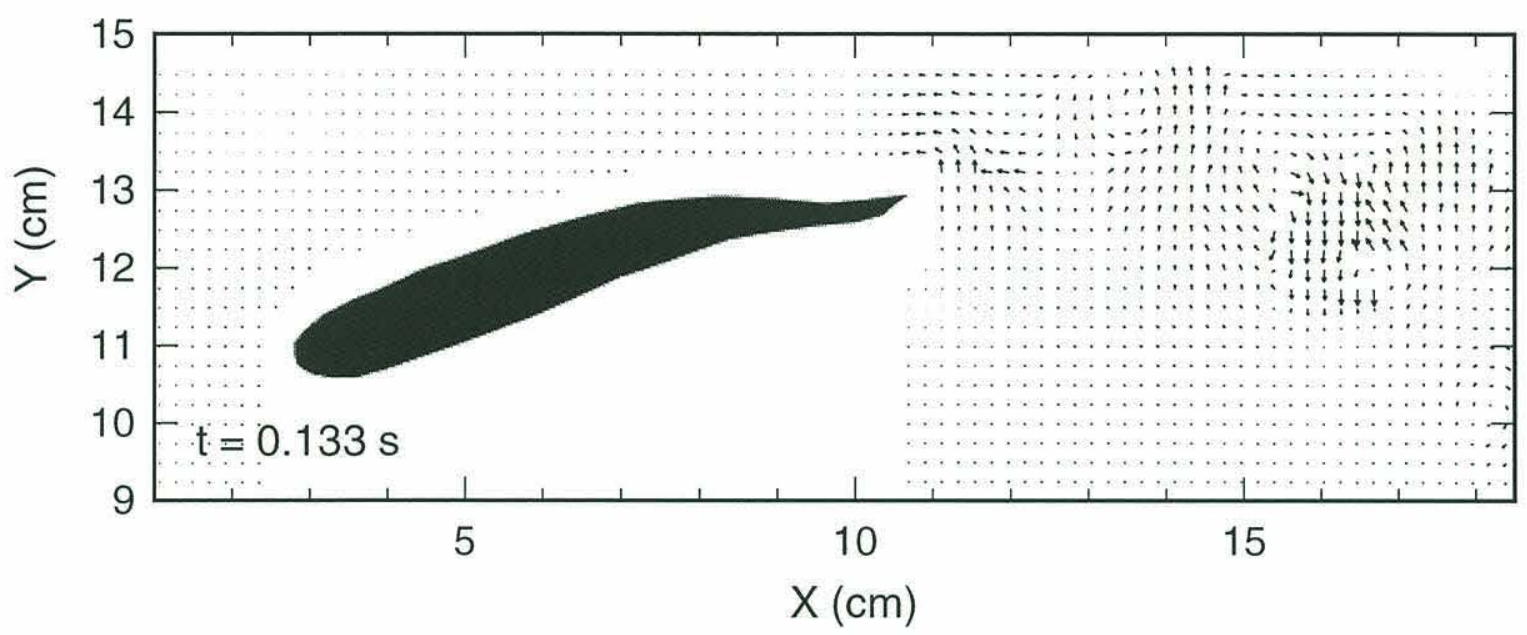

Figure 3-10: Velocity field in the wake of a swimming fish in shallow water.

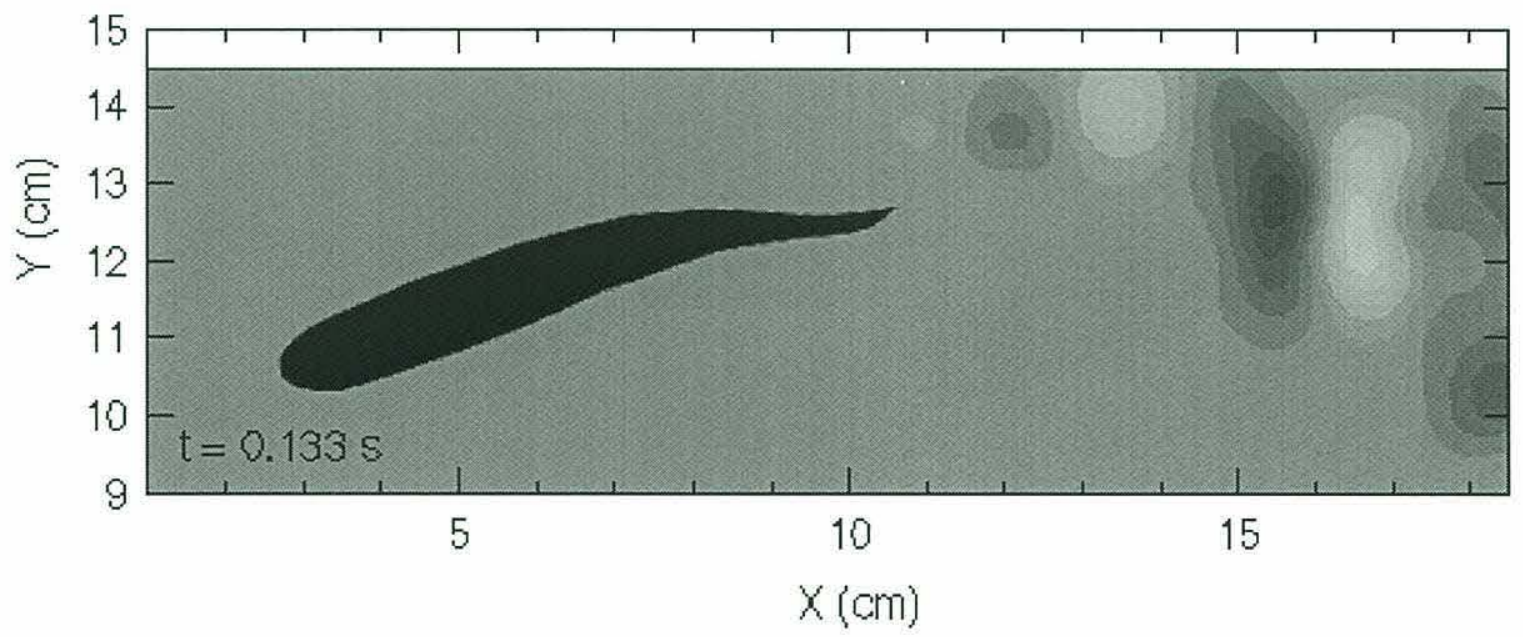

Figure 3-11: Vorticity field in the wake of a swimming fish in shallow water. Contours are from -10.0 to $10.0 \mathrm{~s}^{-1}$ in $1.0 \mathrm{~s}^{-1}$ increments. 


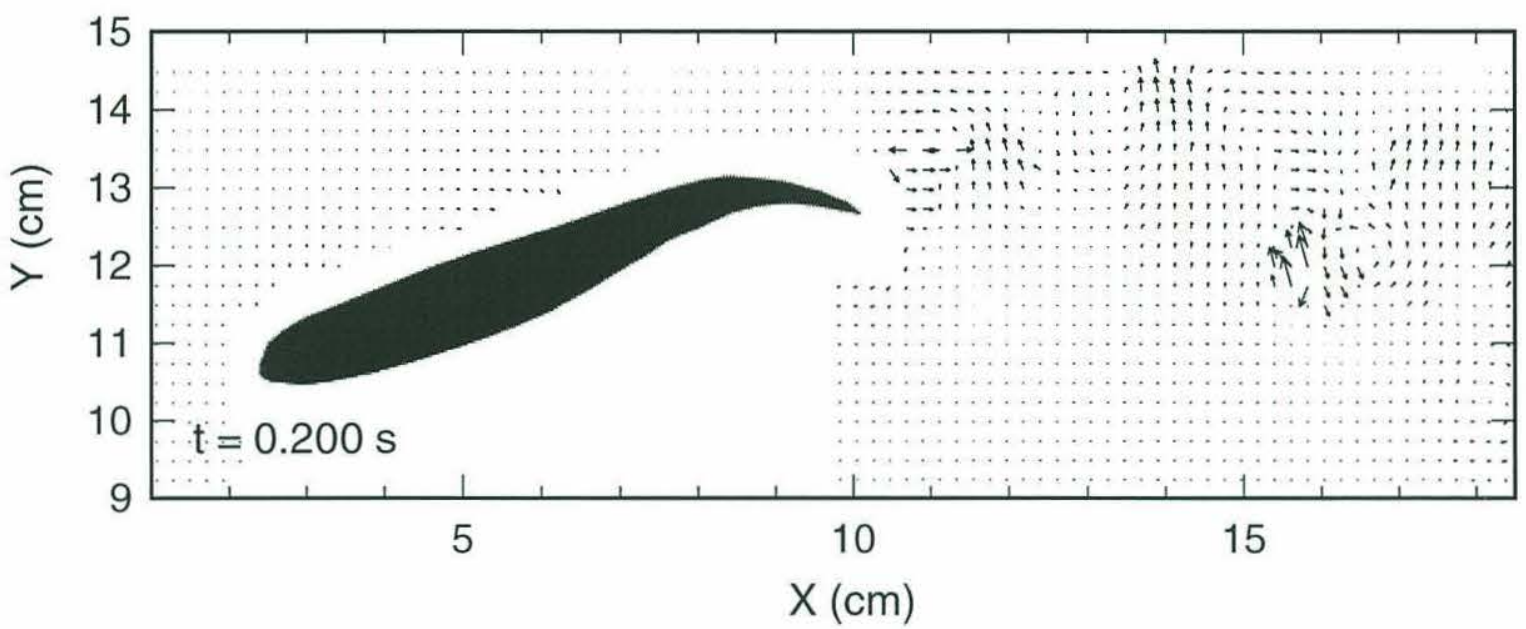

Figure 3-12: Velocity field in the wake of a swimming fish in shallow water.

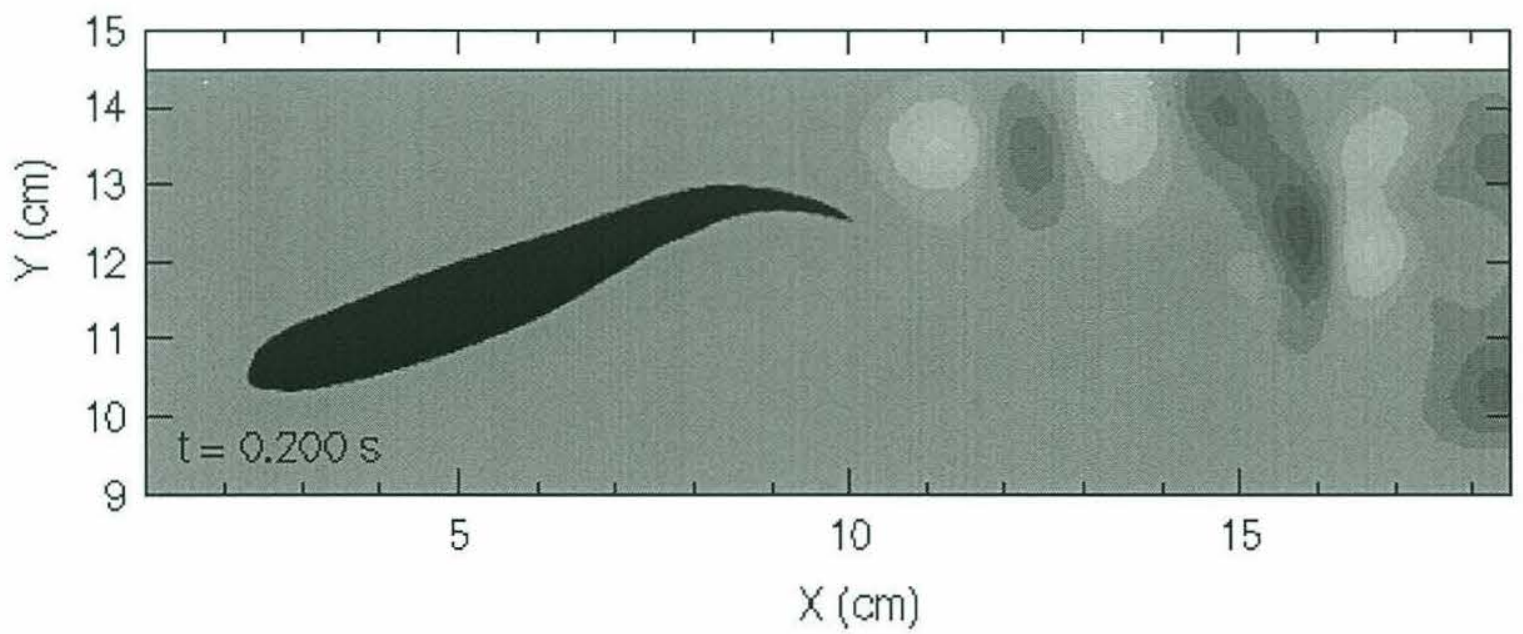

Figure 3-13: Vorticity field in the wake of a swimming fish in shallow water. Contours are from -10.0 to $10.0 \mathrm{~s}^{-1}$ in $1.0 \mathrm{~s}^{-1}$ increments. 


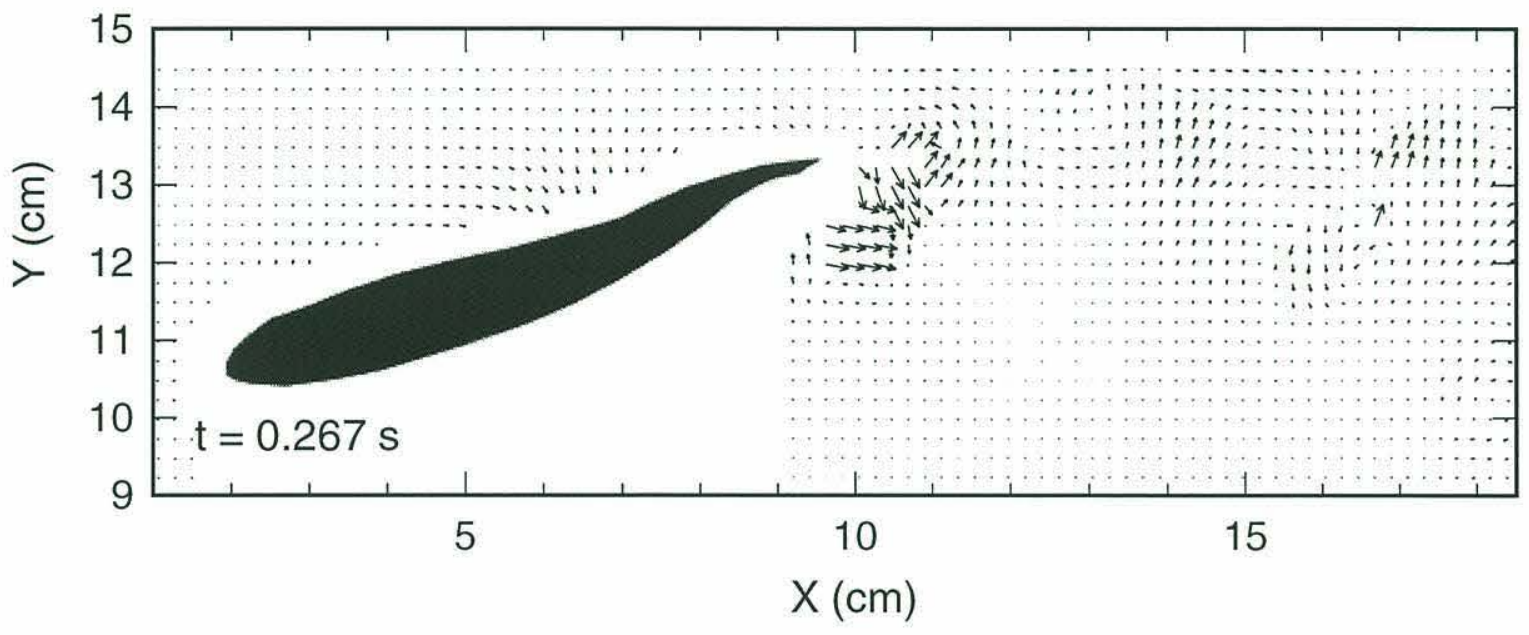

Figure 3-14: Velocity field in the wake of a swimming fish in shallow water.

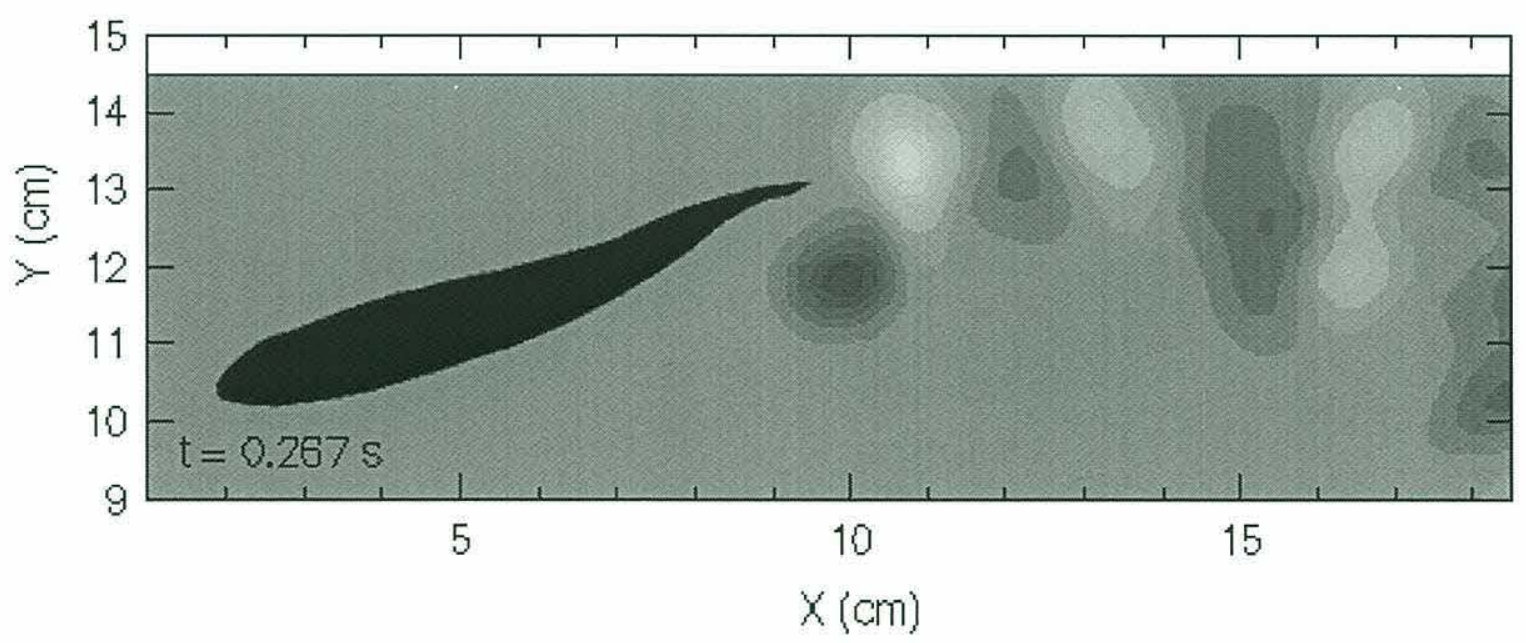

Figure 3-15: Vorticity field in the wake of a swimming fish in shallow water. Contours are from -10.0 to $10.0 \mathrm{~s}^{-1}$ in $1.0 \mathrm{~s}^{-1}$ increments. 


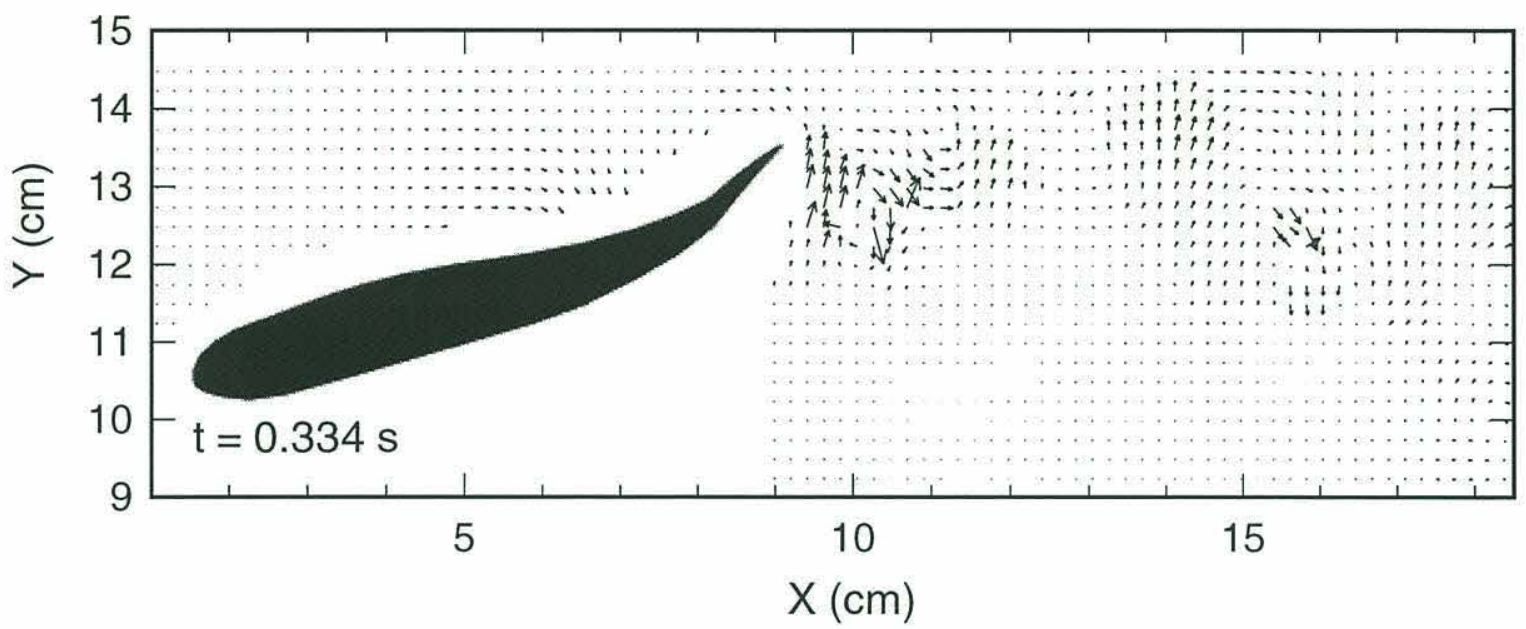

Figure 3-16: Velocity field in the wake of a swimming fish in shallow water.

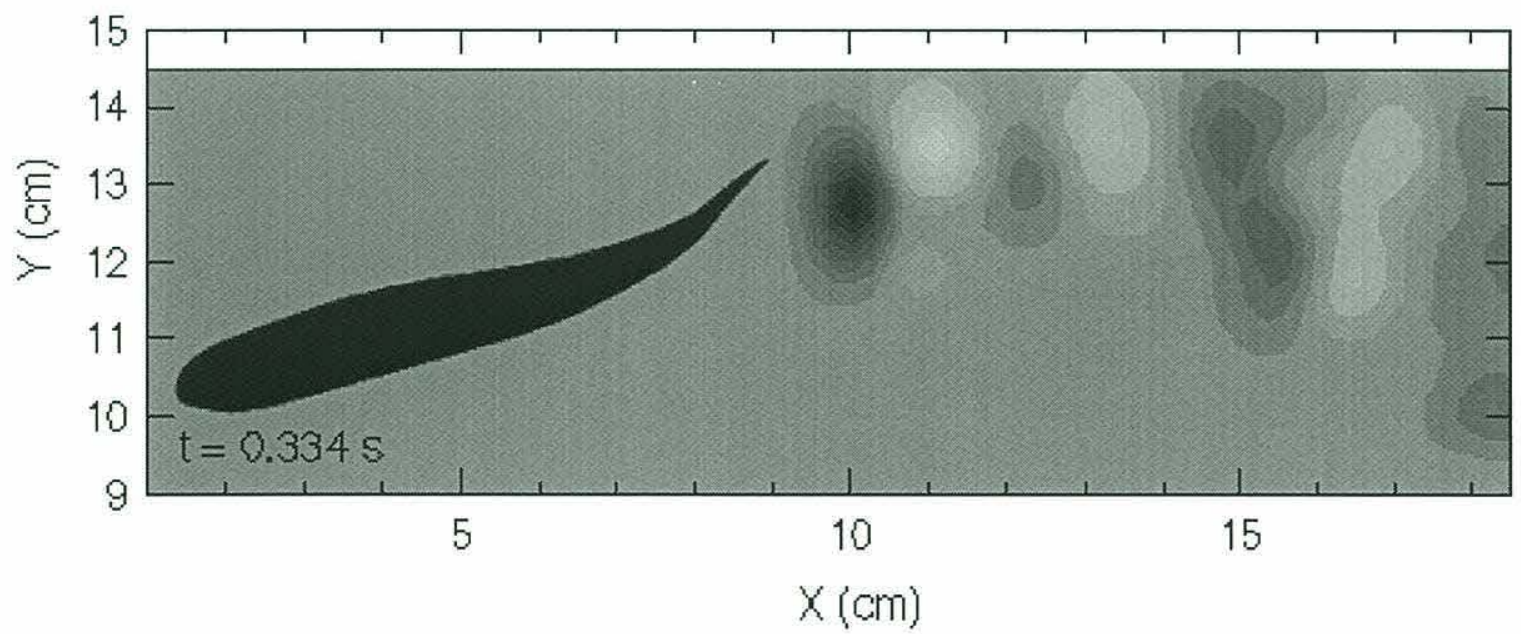

Figure 3-17: Vorticity field in the wake of a swimming fish in shallow water. Contours are from -10.0 to $10.0 \mathrm{~s}^{-1}$ in $1.0 \mathrm{~s}^{-1}$ increments. 


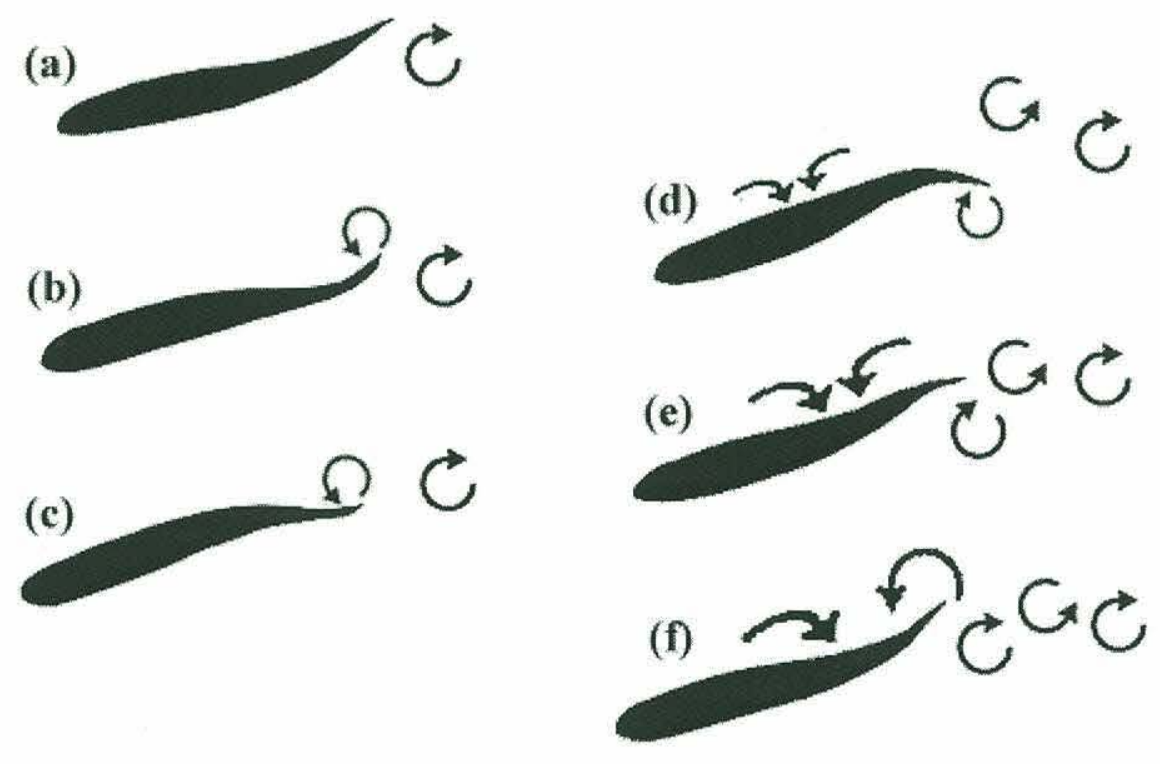

Figure 3-18: Summary of observed flow around a straight swimming fish. (a) starting position with tail up, (b) downward kick begins shedding of vortex at tail, (c) newly formed vortex leaves tail near center of stroke, (d) begin of upstroke starts shedding new vortex at tail. First sign of body wave effect at mid-body. (e) new vortex shed from tail while body wave effect moves posteriorly with amplification. (f) body wave effect concentrates at tail, oriented such that subsequent downstroke will contribute towards tail vortex generation. 
travelling wave is not readily apparent by eye. In anguilliform swimming, the travelling wave has large, relatively constant amplitude. Gray [42] introduced the notion that fluid particles in the vicinity of the crests and troughs of the propulsive wave follow curved trajectories. Figure 3-19 shows a flow visualization of a swimming eel in which at least one wavelength of the travelling wave can be seen. Flakes on the water surface reveal distinct vortices arranged in a jet wake as well as a circular region of fluid which slightly lags the crests of the body-wave.

We demonstrate the mechanism by which this type of flow originates with a simple example of a travelling wave along a fluid boundary: a linear gravity wave in an ideal fluid. If the elevation of the free surface is given by

$$
\eta(x, t)=A \cos (k x-\omega t)
$$

where $A$ is the wave amplitude, $x$ is the spatial coordinate positive in the direction of wave propagation, $k$ is the wave number, $\omega$ is the frequency and $t$ is time. The potential function for this flow in deep water is given by Newman [79] as

$$
\phi(x, y, t)=\frac{g A}{\omega} e^{k y} \sin (k x-\omega t)
$$

where $y$ is the spatial coordinate positive upwards with the origin at the undisturbed free surface. Equation (3.3) uses the deep water dispersion relation, $\omega^{2}=g k$, where $g$ is gravity. The horizontal and vertical velocity components are then given by

$$
\begin{aligned}
& u=\frac{\partial \phi}{\partial x}=\omega A e^{k y} \cos (k x-\omega t) \\
& v=\frac{\partial \phi}{\partial y}=\omega A e^{k y} \sin (k x-\omega t)
\end{aligned}
$$

These equations indicate that the fluid particles move in circular orbits with diminishing orbit radius far away from the free surface. Now, if we superpose a second plane wave on top of the original wave moving in the opposite direction, we obtain the potential of the flow field near a zero thickness, undulating boundary in an inviscid, irrotational fluid.

This flow field is plotted in Figure 3-20 for a wave travelling from left to right. Curved streamlines form in the crests and troughs of the wave which would resemble discrete vortices 
in the absence of the body. This contrived example illustrates the bound vorticity associated with an undulating thin boundary. Outside of the boundary layer, the flow is completely irrotational. At the crests and troughs of the wave, the horizontal velocity above and below the boundary is oppositely signed $\left(\frac{\partial u}{\partial y} \neq 0\right)$ which indicates that vorticity is bound to the interface at these locations. Presumably, if the boundary is instantaneously removed, the two regions would join, and under the action of viscosity become distinct free vortices. This is precisely the mechanism used by a swimming fish. As the undulation wave passes along the body, bound vorticity progresses towards the trailing edge forming distinct vortices which are amplified by shedding at the tail trailing edge.

Experiments done by Taneda and Tomonari [115] on the flow near a waving plate show exactly the same result. Figure 3-21 shows that fluid particles in the troughs and crests of the travelling wave, move along curved paths, in counterclockwise and clockwise directions respectively, for a wave moving from left to right. With the addition of streamwise velocity, the flow cell structure depends on the ratio of the wave speed $c$ to the free stream velocity $U$. When $\frac{c}{U}<1$, the cells separate into free vortices located in the troughs of the waves. For $\frac{c}{U}>1$ (the general case for fish swimming), the particle trajectories flatten, losing their

circular shape. The wave crests and troughs have little effect on the streamlines away from the boundary.

\subsection{Deep water DPIV experiments on straight swimming fish}

The success of the shallow water experiments encouraged us to proceed to deeper water to verify the observed flow, both in the wake and the travelling wave effect near the body. The objectives of this experiment were to ensure that bottom effects do not amplify or distort the observed wake.

Deep water presents us with some difficulty in confining the fish to swim in the horizontal DPIV image plane. The goal was to confine the fish, yet permit the fluid to move freely through the constraint. Another important consideration is that the optical path to the camera must be relatively free of obstruction.

First, we "sandwiched" the fish into the DPIV plane with two wire meshes. A steel mesh constructed out of $2 \mathrm{~mm}$ diameter wire with $2.5 \mathrm{~cm}$ openings provided the necessary rigid 


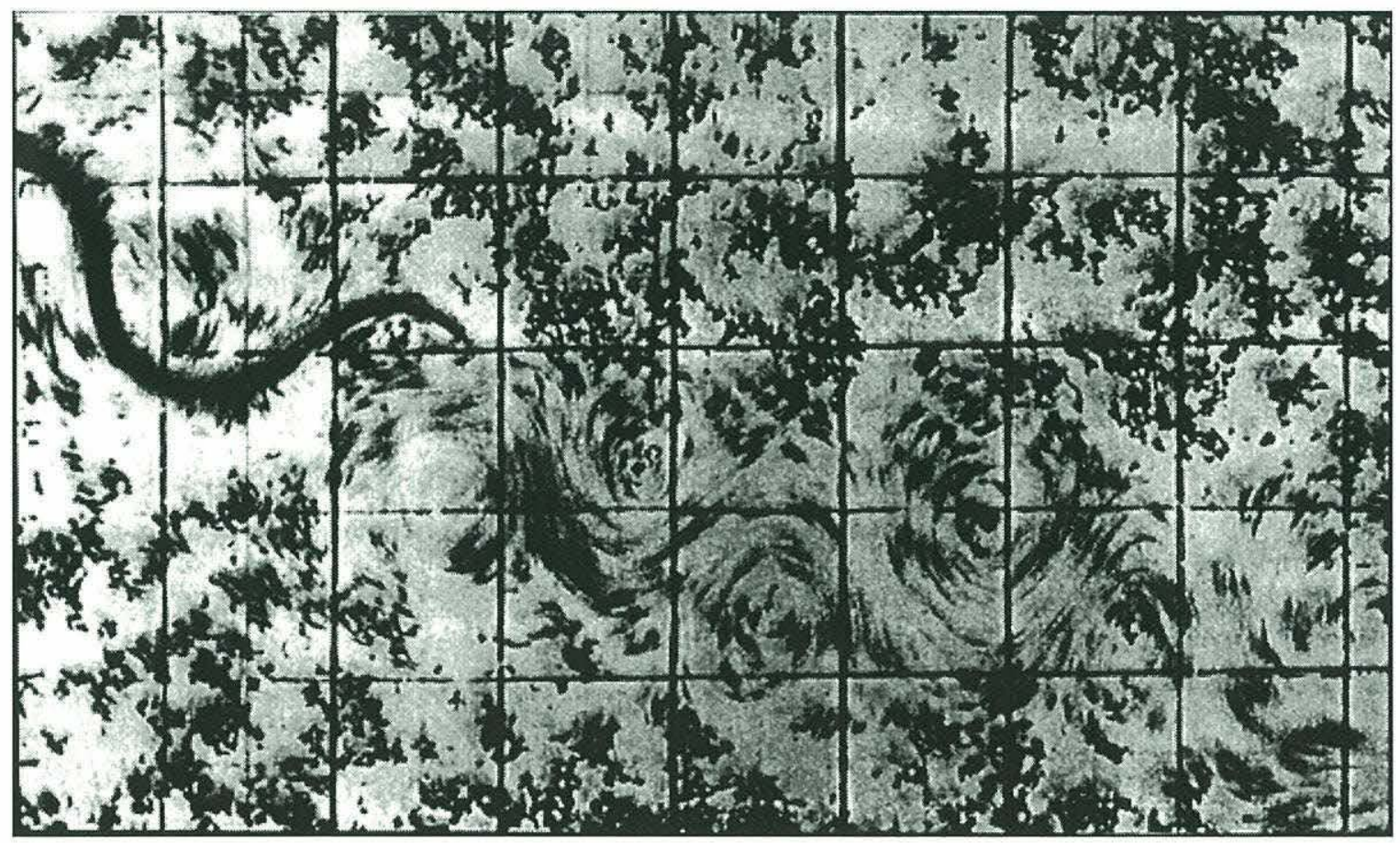

Figure 3-19: Flow around and in the wake of small (16 cm long) eel from Gray (1968).

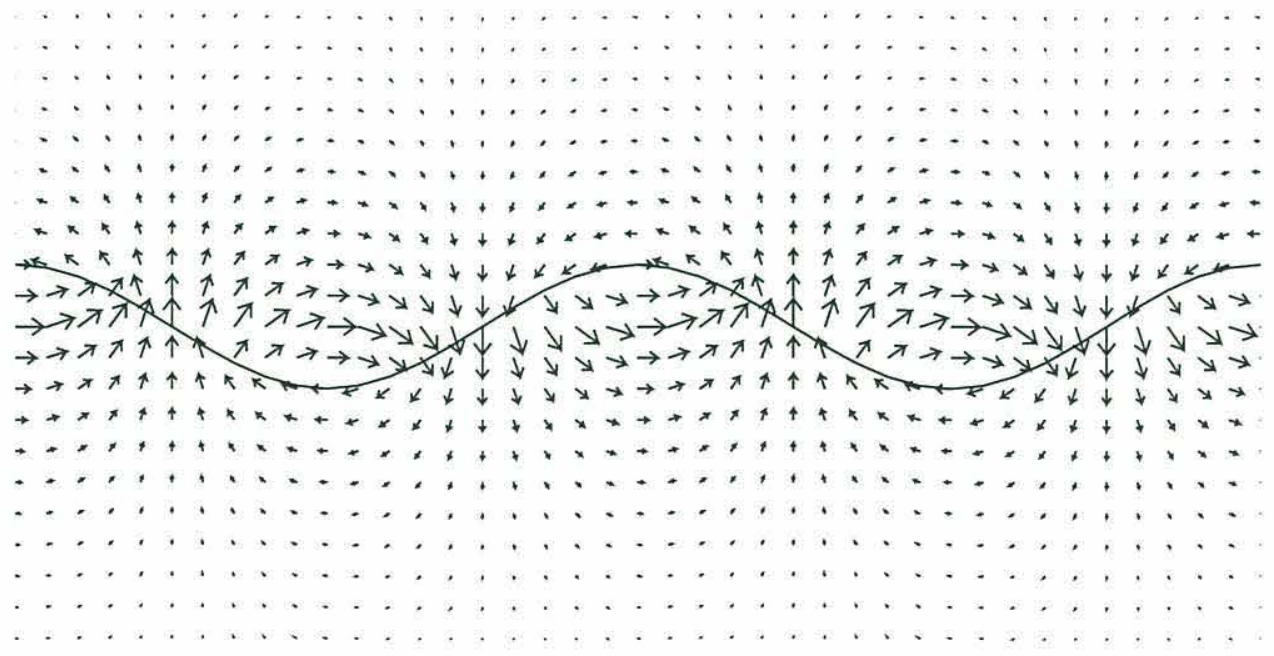

Figure 3-20: Flow near a travelling wave moving from left to right. 


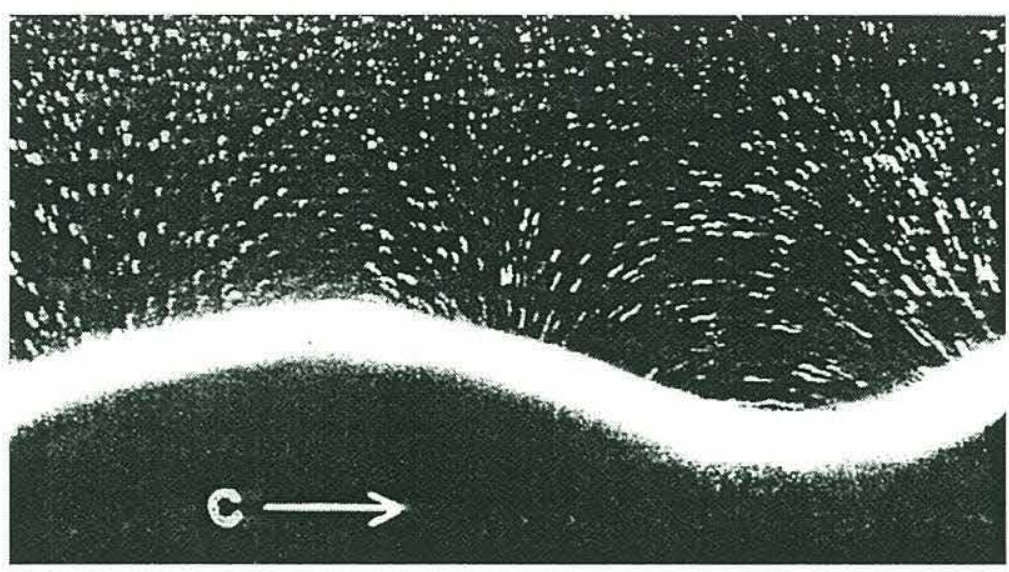

Figure 3-21: Visualization of flow near a travelling wave in still fluid from Taneda and Tomonari (1974).

structure. The mesh was covered with extremely fine nylon netting which confined the fish, yet was virtually invisible to the camera. The entire apparatus was painted matte black and placed at the mid-depth of a $28 \mathrm{~cm}$ deep tank. The opening between the sandwich planes was $3.6 \mathrm{~cm}$ and they occupied an area roughly $30 \times 25 \mathrm{~cm}$.

Although the fish responded well to the deep water apparatus, the mesh in the camera view precluded the use of DPIV processing. However, some wake structure data was obtained by analyzing the video record manually. Consequently, the deep water sandwich experiments were abandoned for a semi-deep approach.

The apparatus was modified to confine the fish between a bottom mesh and the free surface instead of a top mesh. The thickness of the layer was roughly $3 \mathrm{~cm}$. The area of the layer was increased to $51 \times 25 \mathrm{~cm}$ and the depth was kept at $28 \mathrm{~cm}$.

\subsubsection{Results and discussion}

Figures 3-22 through 3-31 show the velocity and vorticity results for the semi-deep case. Again, one complete propulsion cycle is shown, this time with the tail beginning in the maximum downward position. Unlike in the shallow water experiments, the fish swam directly away from the laser light which, to our good fortune, cast a shadow directly upstream of the fish only. Hence, we were able to collect data from both sides of the fish simultaneously. The semi-deep water greatly improved the quality of the results by reducing the divergence.

At the start of the swimming cycle in Figure $3-22$, the fish tail is in the maximum 
downward position. A clockwise vortex is forming with its center near the tip of the tail as a result of bound vorticity associated with the body undulation. The vortex is already well formed although at this point the tail has not begun its upward motion. Subsequent upward tail motion will contribute same-signed vorticity to this vortex. Immediately below the fish and slightly anterior to the tail, the flow follows the upward velocity of the body. Slightly upstream of this position at approximately the mid-body of the fish, longitudinal flow towards the tail marks the formation of the next counterclockwise vortex.

In Figure 3-24, the tail has moved upward and the new clockwise vortex is now clearly visible in the wake. The region of longitudinal flow has moved rearward and upward with the tail motion. In Figure 3-26, the tail is in its maximum position ready to begin the downstroke. The flow on the upper side of the fish follows the body motion downward which defines the circular flow that is to become the next counterclockwise vortex. Figure 3-28 shows this new vortex in the wake as well as the next clockwise vortex forming along the body. In Figure 3-30, the cycle concludes with the tail in the maximum downward position and the next clockwise vortex positioned at the tail tip, ready to be shed on the upstroke.

Figure 3-32 shows the flow in Figure 3-28 magnified in the tail region. This particular instant clearly shows the formation of clockwise flow that is to become the next wake vortex. The entire sequence and flow structure are illustrated in Figure 3-33. The passage of the wave along the body is marked by longitudinal flow on both sides of the fish in the same sense as our wave example (Figure 3-20). The wave induced flow on each side is most pronounced when the body is fully extended to the opposite side (in a trough viewed from each side rather than a crest). This effect is apparent in Taneda and Tomonari's experiment (Figure 3-21) and our simple wave example (Figure 3-20).

Flow properties of the semi-deep case were quantified by averaging over one swimming cycle. The maximum wake velocity along the wake centerline was computed by averaging the streamwise velocity at several locations over one wavelength downstream, at one instant in time. The maximum wake velocity was $0.88 U$ where $U$ is the fish swimming speed, 1.1 body lengths per second. In the regions near the body where the undulation wave has the most effect, the maximum longitudinal (streamwise) velocity component was approximately $0.14 U$ and the maximum transverse velocity (towards or away from the body in the normal direction) was approximately $0.39 \mathrm{U}$. The circulation of the wake vortices was calculated by integrating along circular contours around several wake vortices using equation (2.5). The 


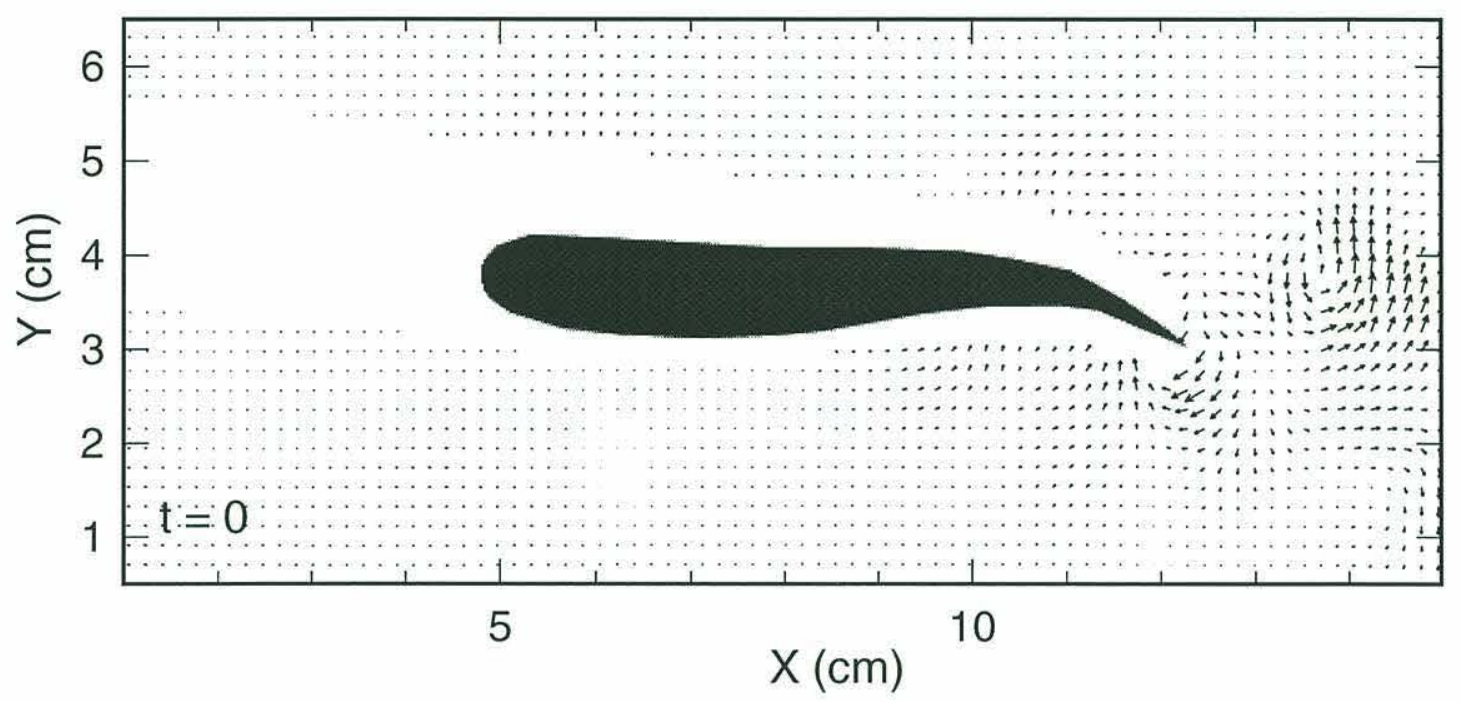

Figure 3-22: Velocity field in the wake of a swimming fish, semi-deep water.

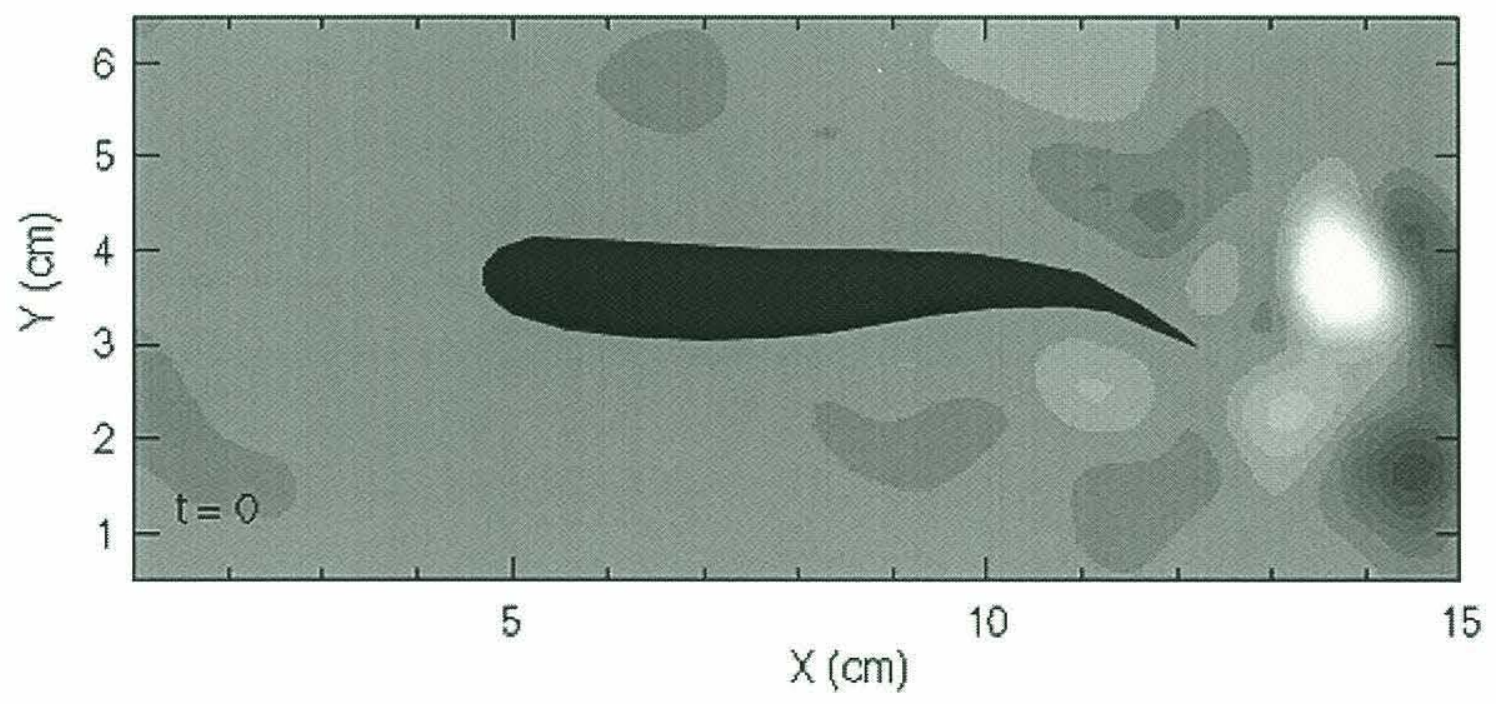

Figure 3-23: Vorticity field in the wake of a swimming fish, semi-deep water. Contours are from -10.0 to $10.0 \mathrm{~s}^{-1}$ in increments of $1.0 \mathrm{~s}^{-1}$. 


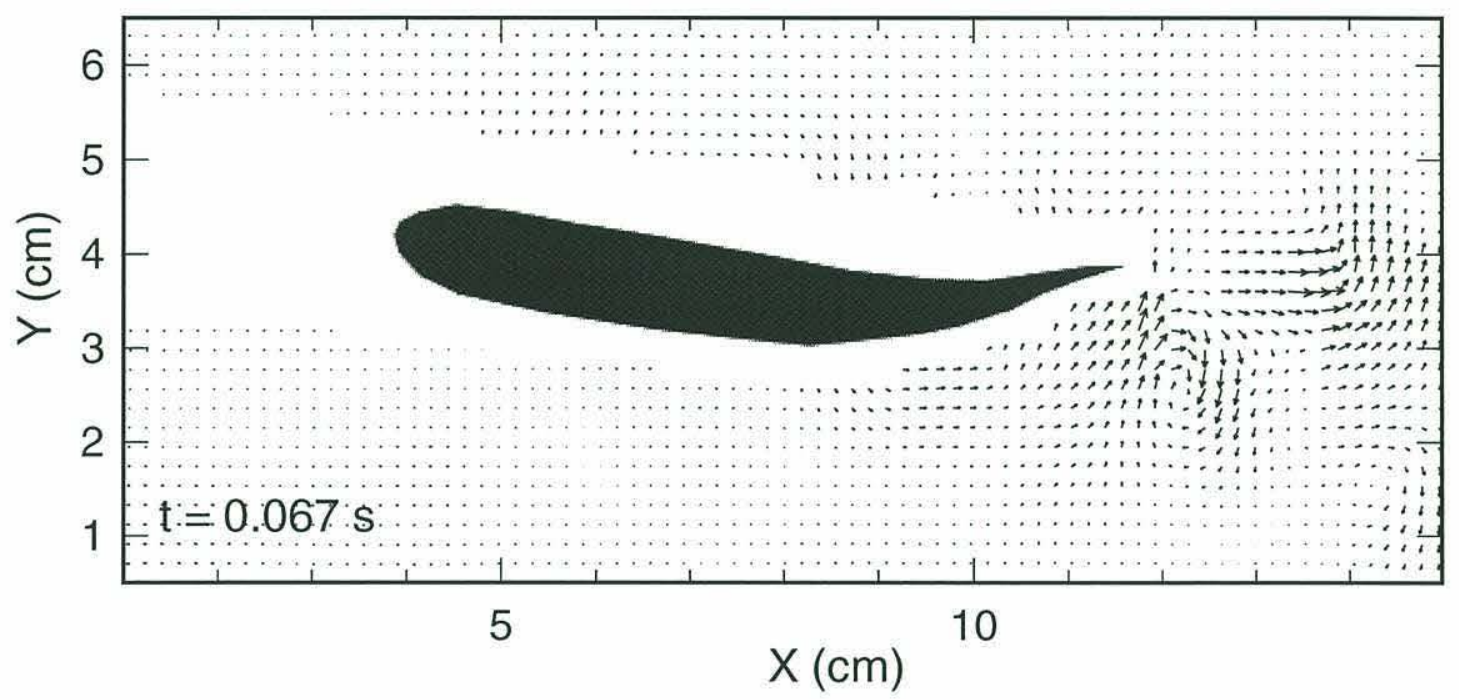

Figure 3-24: Velocity field in the wake of a swimming fish, semi-deep water.

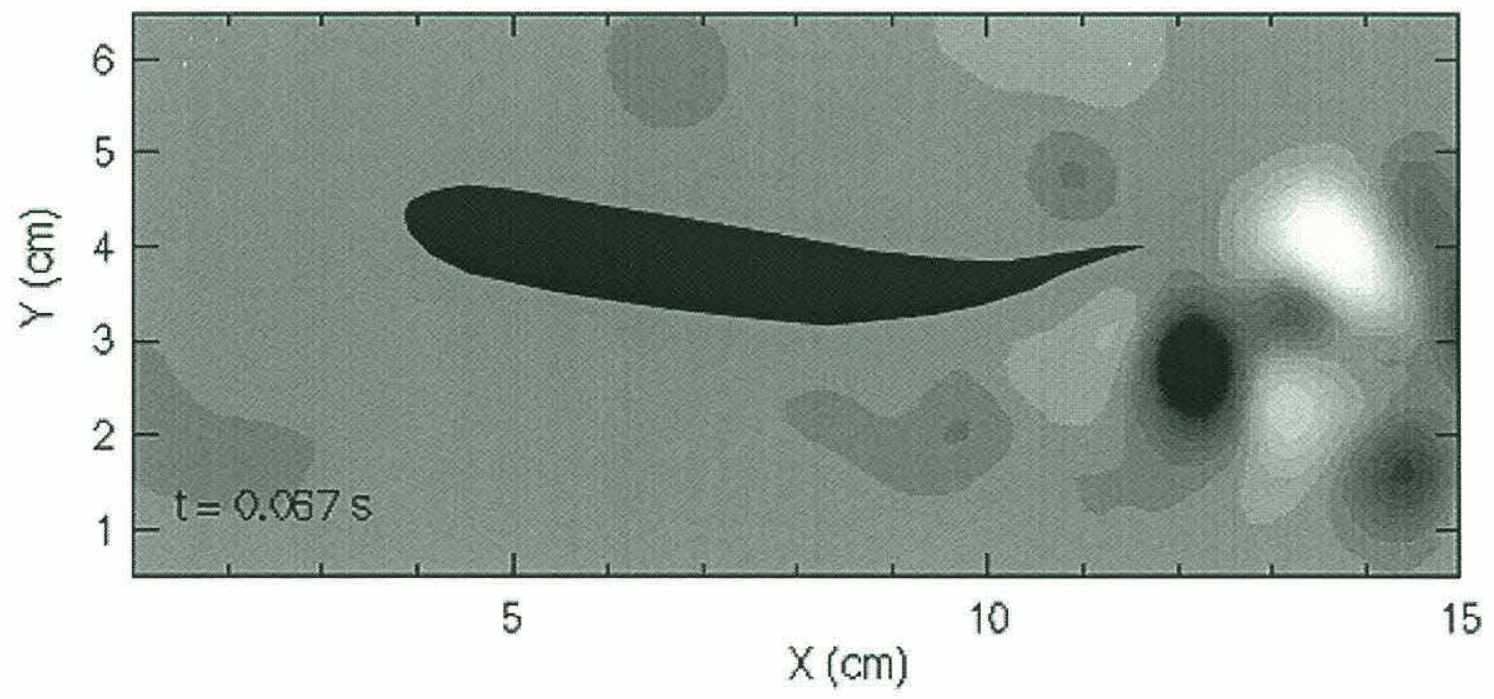

Figure 3-25: Vorticity field in the wake of a swimming fish, semi-deep water. Contours are from -10.0 to $10.0 \mathrm{~s}^{-1}$ in increments of $1.0 \mathrm{~s}^{-1}$. 


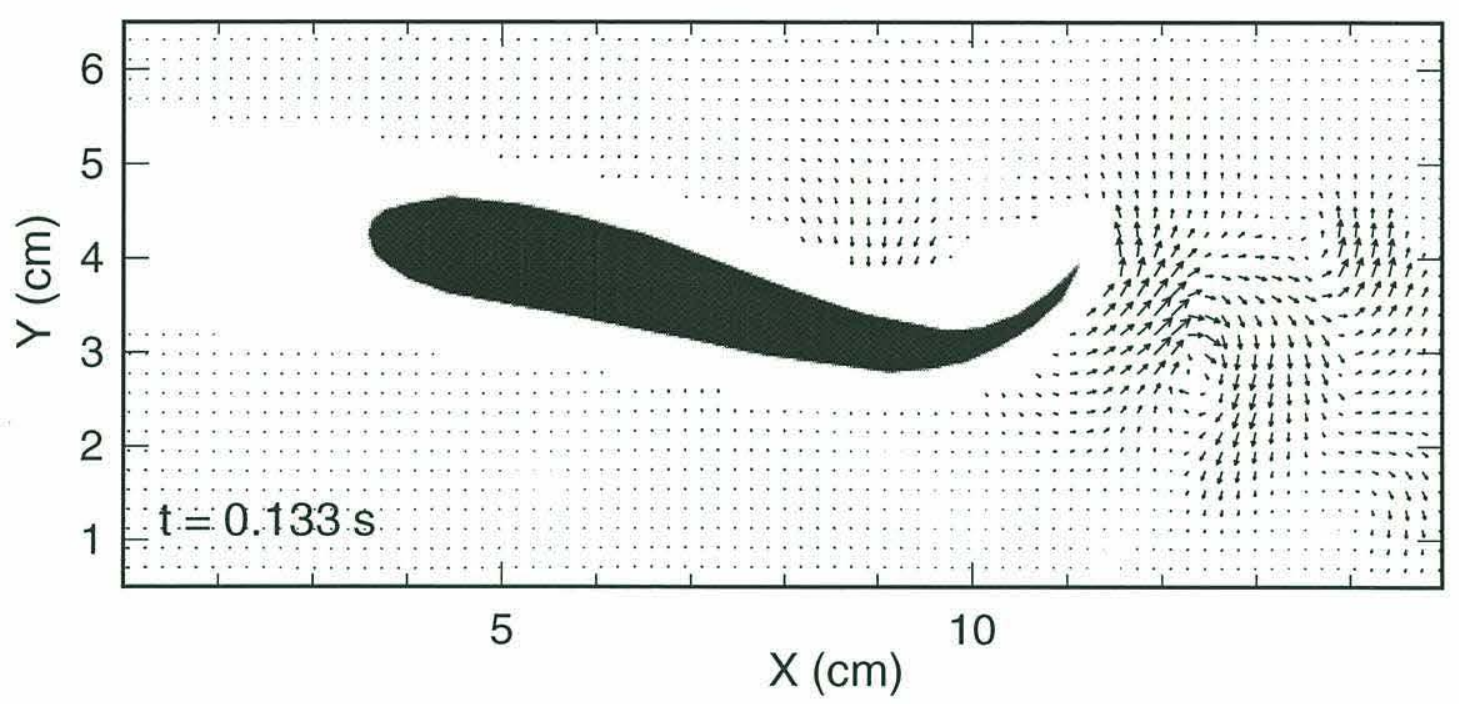

Figure 3-26: Velocity field in the wake of a swimming fish, semi-deep water.

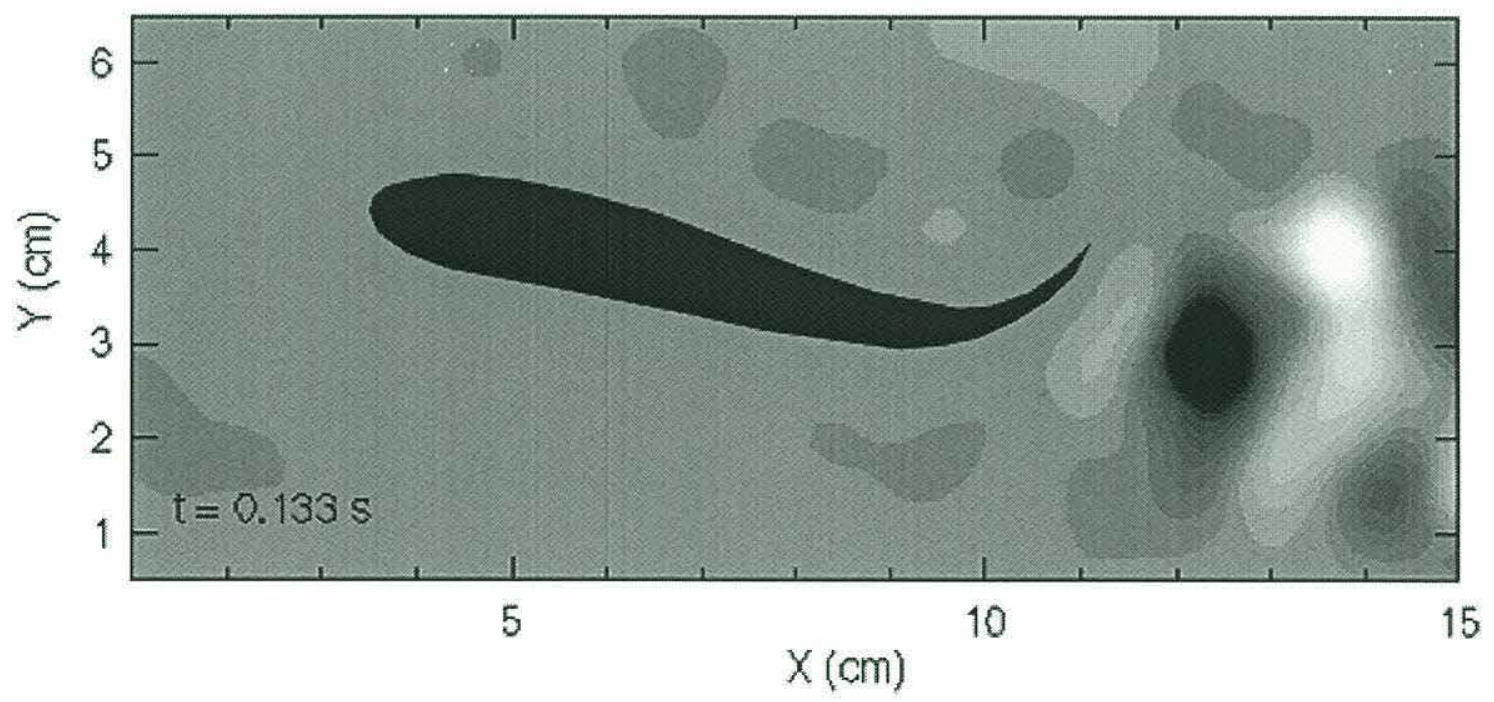

Figure 3-27: Vorticity field in the wake of a swimming fish, semi-deep water. Contours are from -10.0 to $10.0 \mathrm{~s}^{-1}$ in increments of $1.0 \mathrm{~s}^{-1}$. 


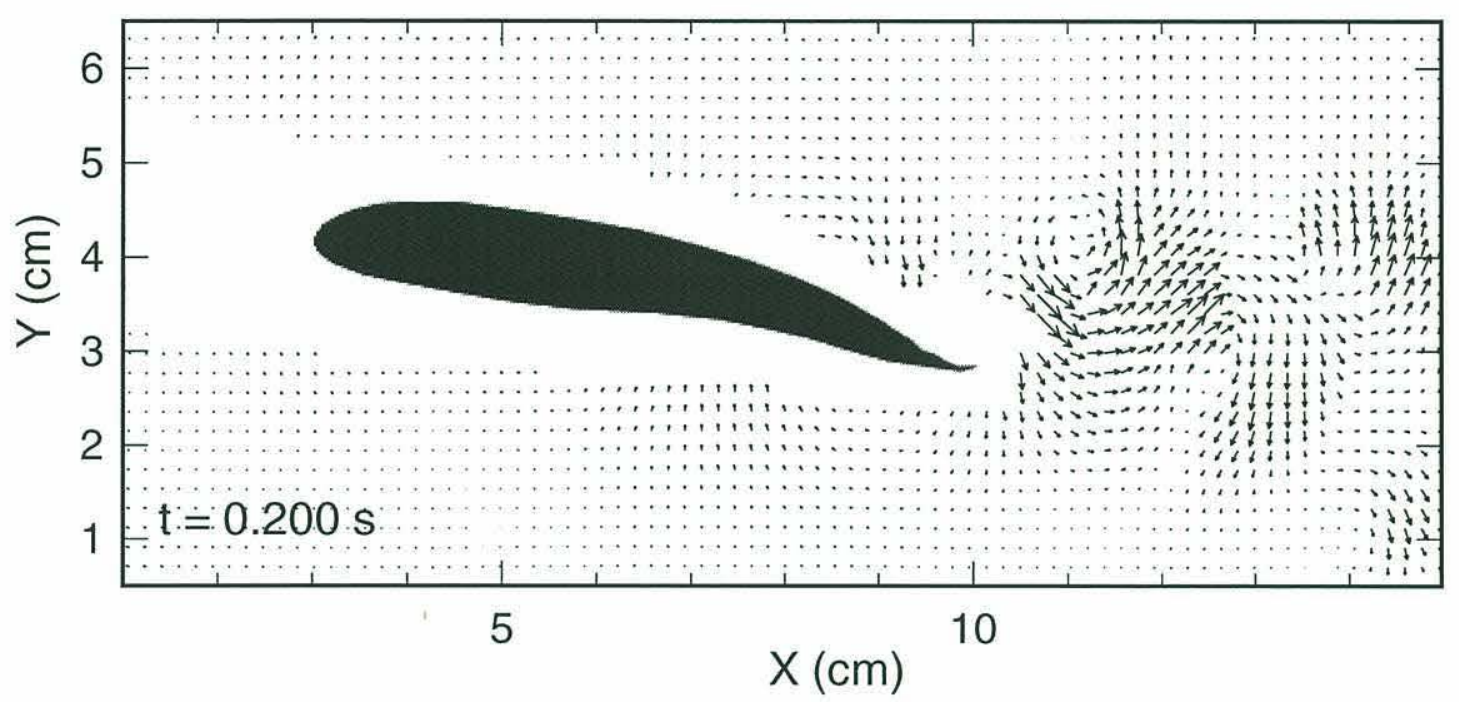

Figure 3-28: Velocity field in the wake of a swimming fish, semi-deep water.

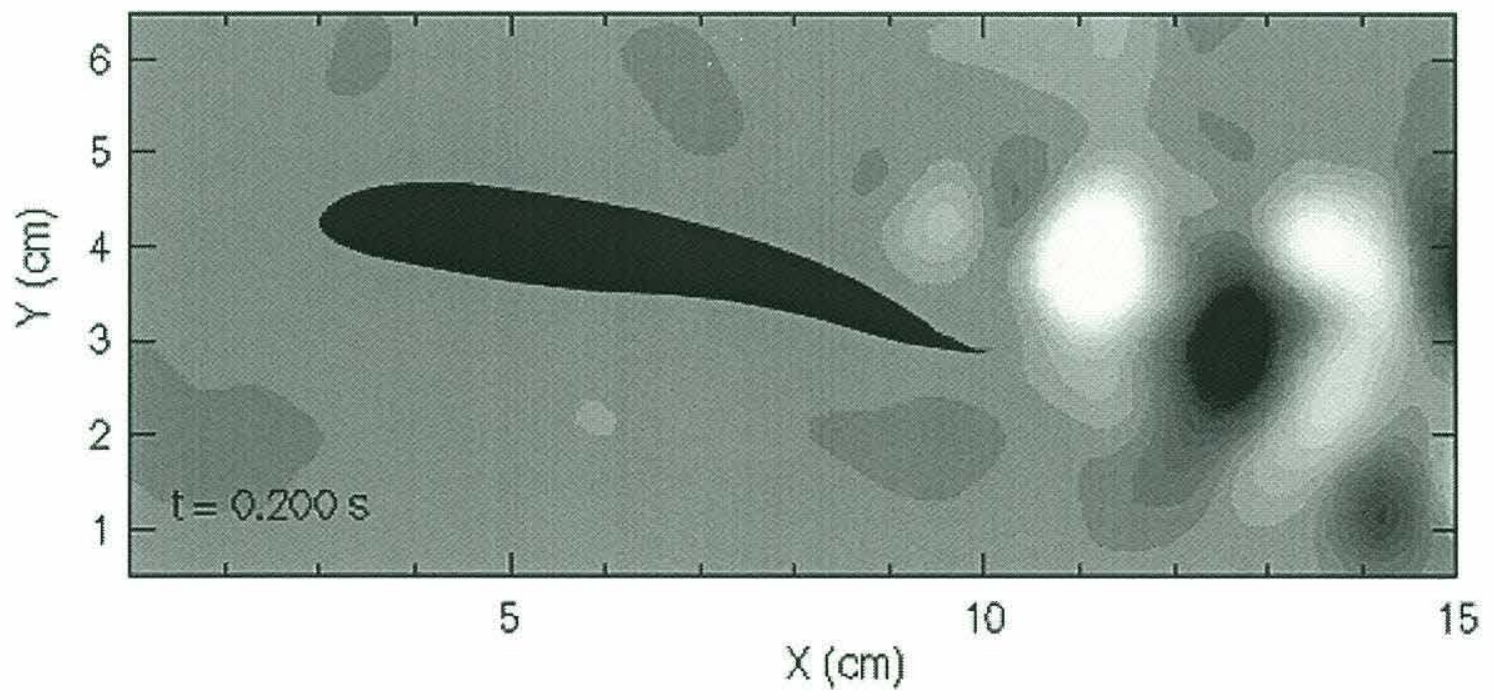

Figure 3-29: Vorticity field in the wake of a swimming fish, semi-deep water. Contours are from -10.0 to $10.0 \mathrm{~s}^{-1}$ in increments of $1.0 \mathrm{~s}^{-1}$. 


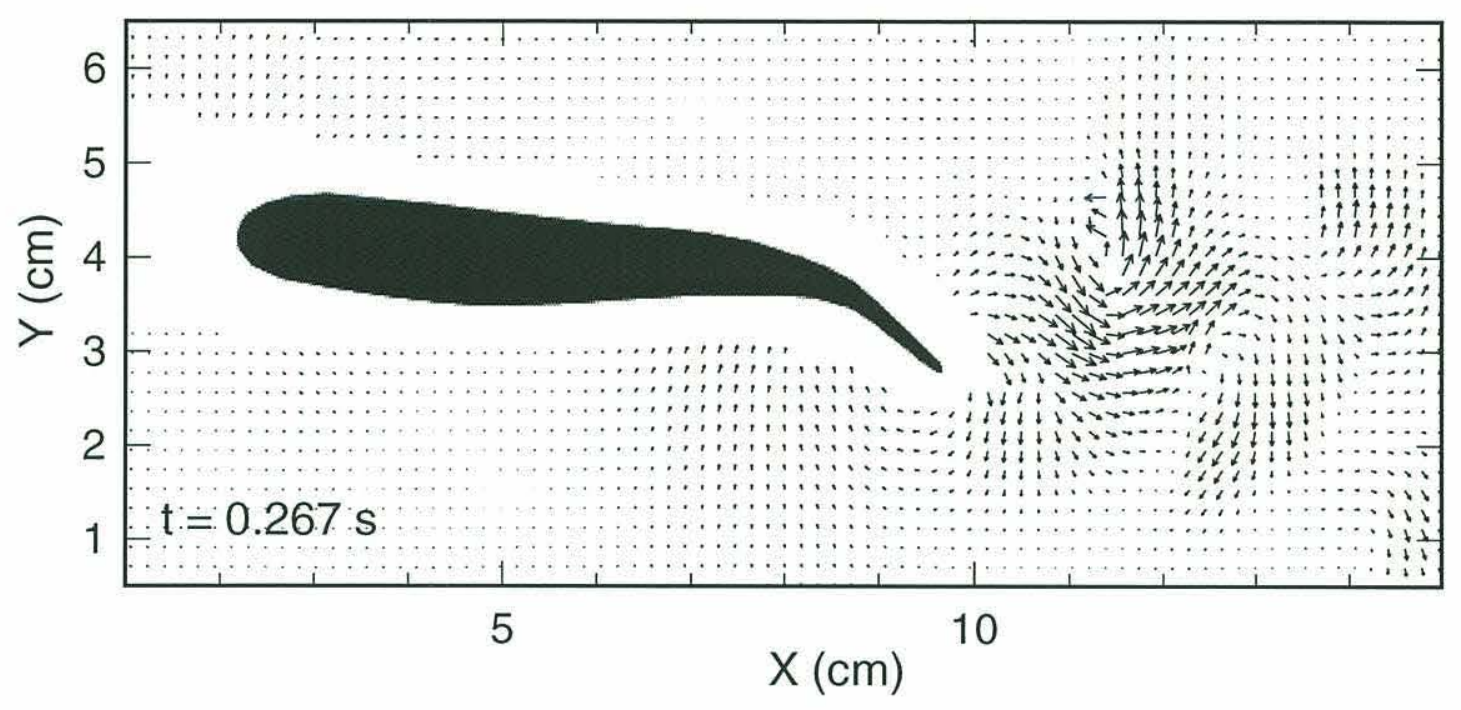

Figure 3-30: Velocity field in the wake of a swimming fish, semi-deep water.

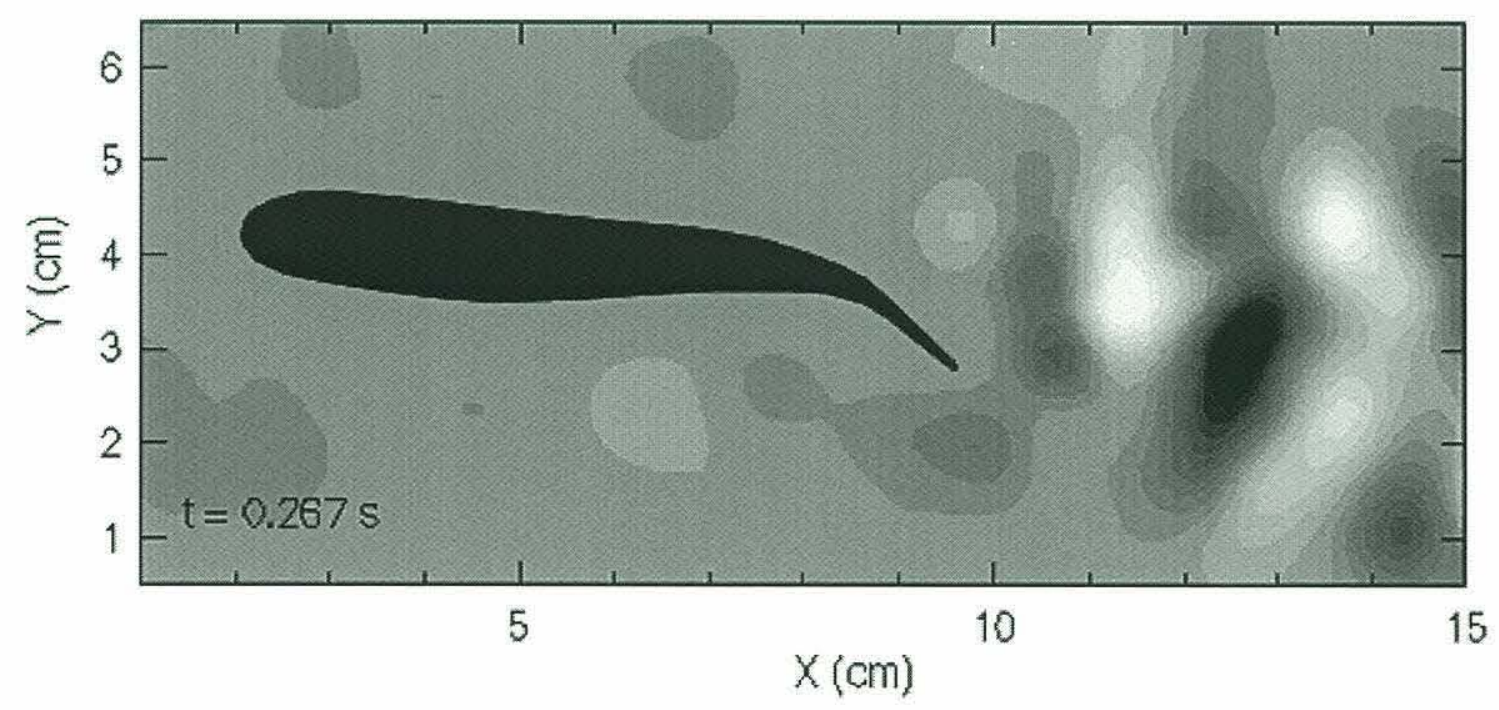

Figure 3-31: Vorticity field in the wake of a swimming fish, semi-deep water. Contours are from -10.0 to $10.0 \mathrm{~s}^{-1}$ in increments of $1.0 \mathrm{~s}^{-1}$. 


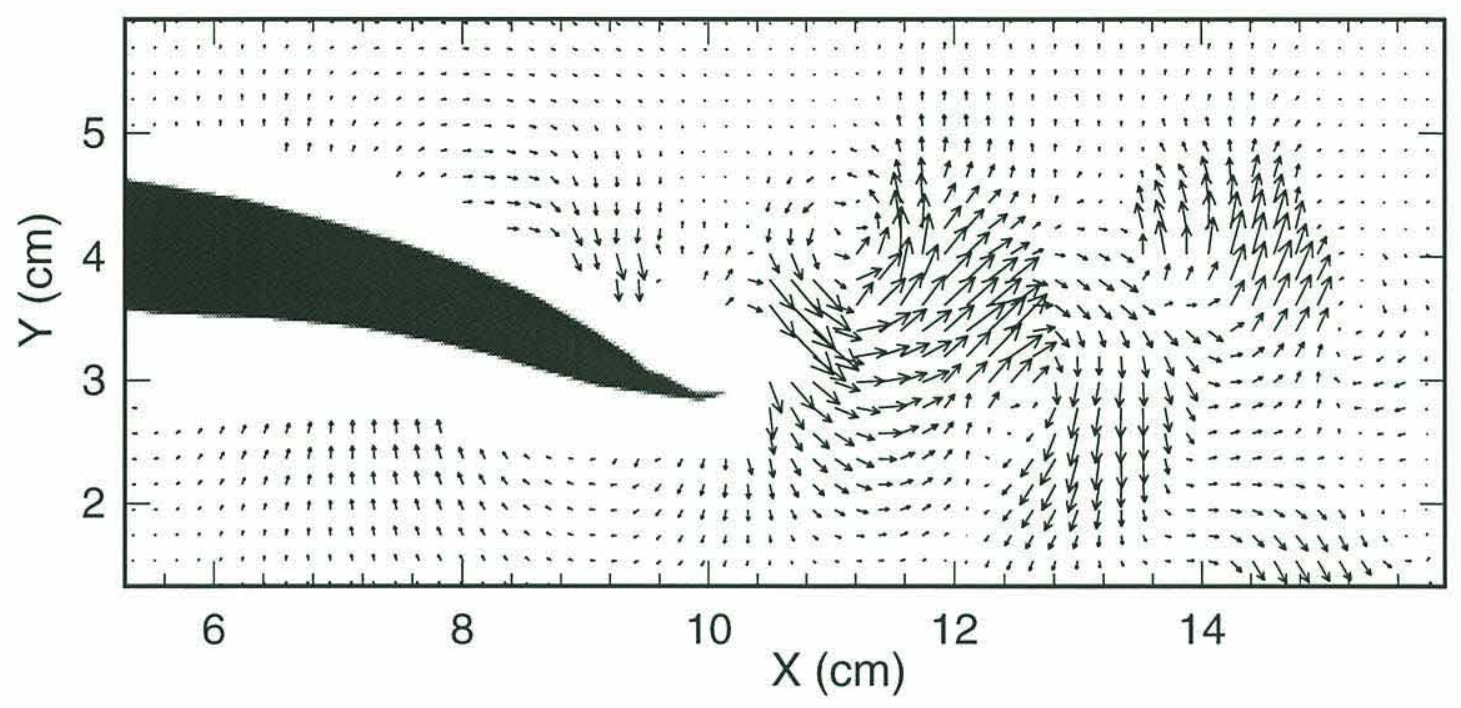

Figure 3-32: Magnification of velocity field near body in Figure 3-28

average circulation was $\frac{\bar{\Gamma}}{\ell U}=0.3$ with core radius $R_{\circ} \approx 0.1 \ell$.

Three-dimensional effects are assessed by computing the normal strain. The normal strain perpendicular to the DPIV plane is

$$
w_{z}=\frac{\partial w}{\partial z}=-\left(\frac{\partial u}{\partial x}+\frac{\partial v}{\partial y}\right)
$$

where $w$ is the out of plane velocity component. Highly divergent flow is indicated by $\left|\frac{w_{z}}{\xi_{z}}\right| \gg 1$ where $\xi_{z}$ is the out of plane vorticity as defined in equation (2.4). In the semideep fish swimming wake, typical values of $\left|\frac{w_{z}}{\xi_{z}}\right|$ are between one and five, indicating a strong velocity gradient in the third dimension. Along the body, typical values are much lower, 0.4 to 0.6 , and are highest in the regions where the flow is perpendicular to the body. These values of normal strain are similar in magnitude to the spanwise normal strain that we observe in Chapter 4 in the wake of a 2-D flapping foil. By comparison, the shallow water fish swimming experiments produced normal strains in excess of ten times the vorticity magnitude. Significant normal strain does not preclude the use of DPIV as long as the correlation peaks are well defined. In our fish experiments, removal of the near wall (below the fish) greatly improved the quality of our correlation peaks by reducing the distortion of the flow due to the wall. Hence, the data have fewer outliers and the wake features are more clearly revealed. 
(a)

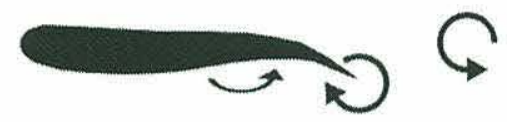

(b)

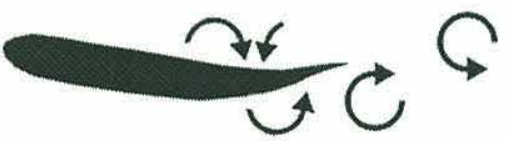

(c)

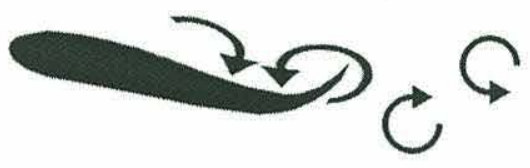

(d)

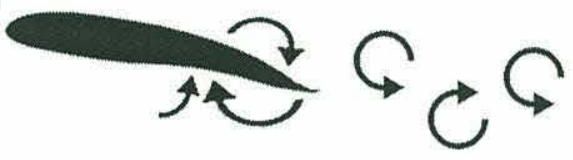

(e)

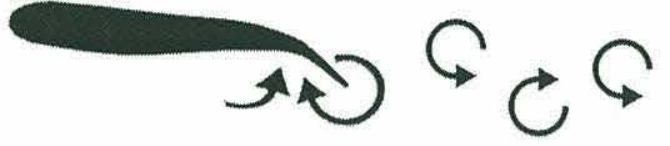

Figure 3-33: Summary of flow around a straight swimming fish in semi-deep water. A full oscillation cycle is shown corresponding to the velocity fields in Figures 3-22 through 3-30. The body-wave effect of Figure 3-18 is seen on both sides of the fish beginning near the fish mid-body. 


\begin{tabular}{||c|c|c|c|c|c|c||}
\hline case & $\frac{A}{\ell}$ & $\begin{array}{c}f \\
(\mathrm{~Hz})\end{array}$ & $\begin{array}{c}U \\
\left(\frac{\ell}{s}\right)\end{array}$ & $\begin{array}{c}S t \\
\frac{A f}{U}\end{array}$ & $\frac{h}{\ell}$ & $\begin{array}{c}S t_{h} \\
\frac{h f}{U}\end{array}$ \\
\hline \hline K-fluid & 0.25 & 7.1 & 5.5 & 0.33 & $0.28^{*}$ & $0.36^{*}$ \\
shallow I & 0.11 & 2.7 & 0.66 & 0.42 & 0.06 & 0.25 \\
shallow II & 0.12 & 3.3 & 0.88 & 0.46 & 0.09 & 0.34 \\
semi-deep & 0.16 & 3.3 & 1.1 & 0.47 & 0.15 & 0.44 \\
deep & 0.21 & 6.2 & 2.3 & 0.55 & $0.22^{*}$ & $0.59^{*}$ \\
\hline
\end{tabular}

Table 3.1: Summary of fish wake parameters. $A$ is the tail double amplitude measured to the tail tip, $f$ is the tailbeat frequency and $U$ is the fish speed. The last column is the adjusted Strouhal number based on wake width $h$ rather than tail excursion. Values indicated with an asterisk are estimated.

\subsection{Kinematic analysis}

In this section we address the kinematics of the fish and the wake structure. Table 3.1 summarizes the measured wake parameters for all of the straight swimming fish experiments. The highest tail amplitude, frequency and speed were observed in the K-fluid visualizations. The Strouhal number for this motion was estimated to be near optimal at 0.33 . In the DPIV experiments, the fish exhibited varied behavior with tail amplitudes between 0.11 and $0.21 \ell$ and frequencies up to $6.2 \mathrm{~Hz}$. Consequently, the Strouhal number shows some variability. Typical relative errors in the analysis of the fish and wake kinematics are $25 \%$ for the tail amplitude measurement, $15 \%$ for the frequency measurement, $5 \%$ for the speed measurement and up to $30 \%$ for the Strouhal number calculation. Much of the error is due to the fact that the fish does not always swim with constant tailbeat amplitude or frequency rather than measurement error. The values in Table 3.1 are averages of data with significant standard deviations from the mean. Considerable improvement could be made in these measurements by acquiring more data to improve the statistical sample.

The wake Strouhal numbers $\left(S t_{h}\right)$ clearly support the conclusions of Triantafyllou et al. [118]. Although our kinematic measurements are approximate, most of the Strouhal numbers are inside the predicted optimal range of $0.25<S t<0.4$. 


\subsection{Summary of straight swimming}

Figure 3-34 (a) summarizes the flow observed in our DPIV experiments in the horizontal plane. Along the fish, bound vortices induce longitudinal and lateral flow near the crests and troughs of the propulsive body-wave. The vorticity in these regions is negligible which indicates that the flow is nearly irrotational. These regions move posteriorly to the tail where they are shed into the wake. Prior to interacting with the tail, the flow is organized into a nearly circular flow which has the same rotation as a vortex that would be produced by subsequent tail motion. Hence, the phase of the undulating wave and caudal movements are matched such that the body-wave aids prepares the formation of discrete wake vortices. Our observations support the conclusion that the formation of discrete vorticity is fundamental to the propulsion process, contradicting the notion that near-wake vortex formation is undesirable.

In Figure 3-34 (a), the caudal fin is shown at its maximum excursion on the right side of the fish. In subsequent motion, the tail sweeps to the left side, amplifying the already forming vortex at the tail tip. In Figure 3-34 (b), heavy lines show a simplified model of the vortex structure along the fish and in the wake. The model is simplistic in that bound vortices along the body and wake vortices are drawn as single filaments. The actual vorticity would be better represented by a lattice of vortex filaments along the body and in the wake. The vortices are connected by streamwise filaments (tip vortices) in the usual sense.

\subsection{DPIV experiments on maneuvering fish}

One of the most exciting topics in fish swimming hydrodynamics is the fast-start/maneuvering capability of fish. Most fish can turn $180^{\circ}$ on a radius considerably less than their body length whereas man-made underwater vehicles require several lengths to execute a $90^{\circ}$ turn. Fishes do not need to be moving in order to turn, nor do they use their tail as a rudder. During fast-starts and maneuvers, the fish body contorts dramatically and recoils so quickly that high speed cinematography is usually required to capture the details of the motion.

Previous work in this area is limited to kinematics and study of musculature employed in the vigorous large amplitude body movements. Gray [41] studied the turning kinematics of whiting and goldfish, in particular, pectoral fin usage and musculature cycles. 


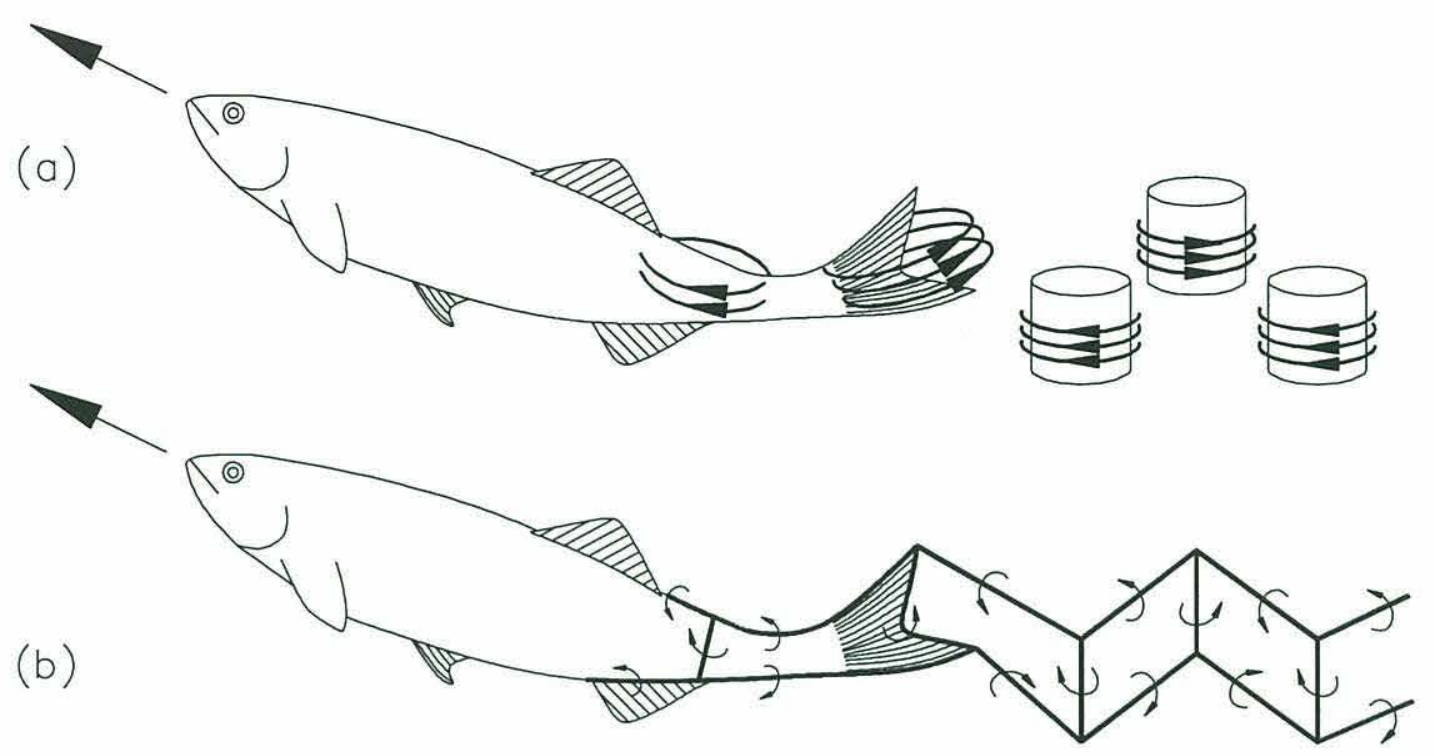

Figure 3-34: Three-dimensional flow structure around a straight swimming fish. (a) velocity field in horizontal planes, (b) vorticity structure.

Weihs [132] demonstrated that fast-start kinematic patterns are divisible into three stages: (i) a preparatory stroke, (ii) a main propulsive stroke and (iii) a variable third stage such as normal swimming or coasting. Maximum acceleration is achieved in the preparatory and main propulsive stroke. During these types of maneuvers, the median fins (dorsal and ventral) are usually fully erected, presumably to increase the lateral cross section to maximize the inertial forces.

Webb $[129,130]$ studied the fast-start performance of several species of teleosts and the relation to morphology. Very high accelerations up to four times gravity were measured during the fast-start main propulsive stroke. Weihs [132] measured the acceleration of pike to be $50 \frac{\mathrm{m}}{\mathrm{s}^{2}}$. Webb found little variation in fast-start kinematics between species; rather, observed differences were correlated to the fish size.

The conventional understanding of fast-starts and maneuvers is that fluid inertia is the dominant factor. The kinematics associated with high average thrust at low average energy loss is the case in which the rear part of the body moves at high speeds, perpendicularly to the intended motion of the fish while oriented with a small angle of attack. Large body depth away from the center of mass where the body movements are maximum is regarded as optimal. For example, known fast predators such as barracudas and pikes have relatively wide flat bodies and large posterior median fins. 
Recent studies by Harper and Blake [46] on the rainbow trout and northern pike confirm that fast-start performance is related to body form, with the pike far superior to the trout due to its deepened posterior body section. Harper and Blake measured acceleration directly (in excess of twelve times gravity) by implanting tiny accelerometers subcutaneously near the fish center of mass as well as inferring acceleration from cinematographic methods. They observed the traditional "L" shape (also referred to as a "C" shape) and a "S" shape preparatory strokes.

We were fortunate to capture a vigorous Danio maneuver with DPIV during the shallow water experiments described in section 3.4. Figure 3-35 shows the trajectory of the turning fish during this segment. The fish begins the maneuver coasting downwards, then suddenly contorts its body into a tight curve and recoils to resume straight line swimming in a direction $105^{\circ}$ towards the right from the starting direction.

The DPIV results for this maneuver are shown in Figures 3-36 through 3-42. The region immediately below the fish is in the shadow of the fish so these data are removed. Before arriving at the position shown in Figure 3-36, the fish completed a turn originating in the upper left corner of Figure 3-36. Residual vorticity from these movements remains above and to the left of the fish. In Figure 3-37, the fish begins to bend, moving its head towards the new swimming direction with the tail moving in the opposite sense, forming the characteristic "L" shape. The fluid follows the body curvature, moving directly towards and away from the fish at the point of maximum curvature at the fish mid-body.

In Figure 3-39, we see the flow organize into two circular flows, one centered at the tail and one near the head. In Figure 3-40, the tail begins to move towards the right, shedding the already formed counter-clockwise vortex. At this instant, the effect of bound vorticity near the head is obscured due to an unfortunate body reflection. The next several frames are not presented due to body reflections which obscure a large portion of the flow. However, careful analysis of the video record shows that during the downstroke of the tail (the main propulsive stroke), the counterclockwise vortex sheds into the wake. During the upstroke, the clockwise vortex initially associated with the head region, moves posteriorly and sheds.

The net result is shown in Figure 3-41: a vortex pair which forms a jet directed slightly downward and to the right. Figure $3-42$ shows the vorticity contours at this instant. The entire turning sequence, from a coasting position to steady swimming $105^{\circ}$ from the starting position takes only half a second. As in our straight swimming measurements, the vorticity 


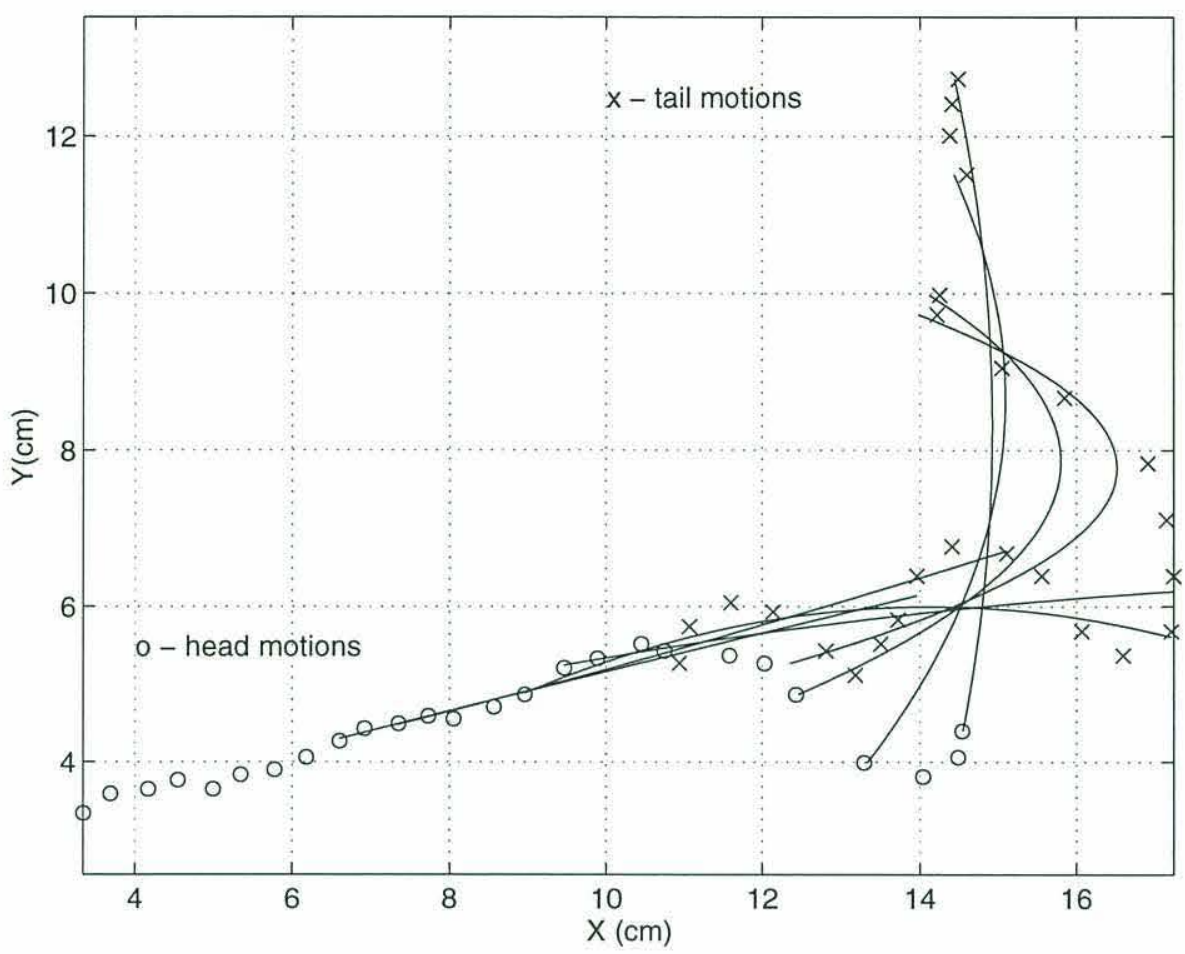

Figure 3-35: Trajectory of maneuvering fish. The 'o' and 'x' marks indicate head and tail motions respectively and the curves represent a few of the body positions. The starting position is vertical with the head pointing downwards. The fish contorts its body into a tight curve, then recoils to resume straight swimming.

is negligible in the regions of induced flow near the body until it is shed into the wake. Figure 3-43 schematically summarizes the body movement and subsequent fluid motion.

After completing the turn, the fish begins to swim steadily at $U=1.5 \frac{\ell}{s}$. The vortices comprising the jet have average nondimensional circulation $\bar{\Gamma}^{*}=\frac{\bar{\Gamma}}{\ell U}=0.43$ which is $42 \%$ greater than the typical wake vortex strength for steady swimming. The core radius of the jet vortices is more than double that of those produced in straight swimming. The jet is $0.34 \ell$ wide, with maximum jet velocity of $u_{j} \approx U$ oriented $105^{\circ}$ to the left of the initial starting position as seen by the fish. Interestingly, the fish velocity after the turn is roughly the same as the jet velocity. Also, the direction of the jet appears to balance the direction of the fish in a horizontal momentum balance. As with the previous shallow water results, three-dimensional effects are not insignificant. The vigorous motion and shallow depth combine to disrupt our results. Unfortunately, this experiment could not be repeated in deep water due to the uncooperative nature of the fish. 


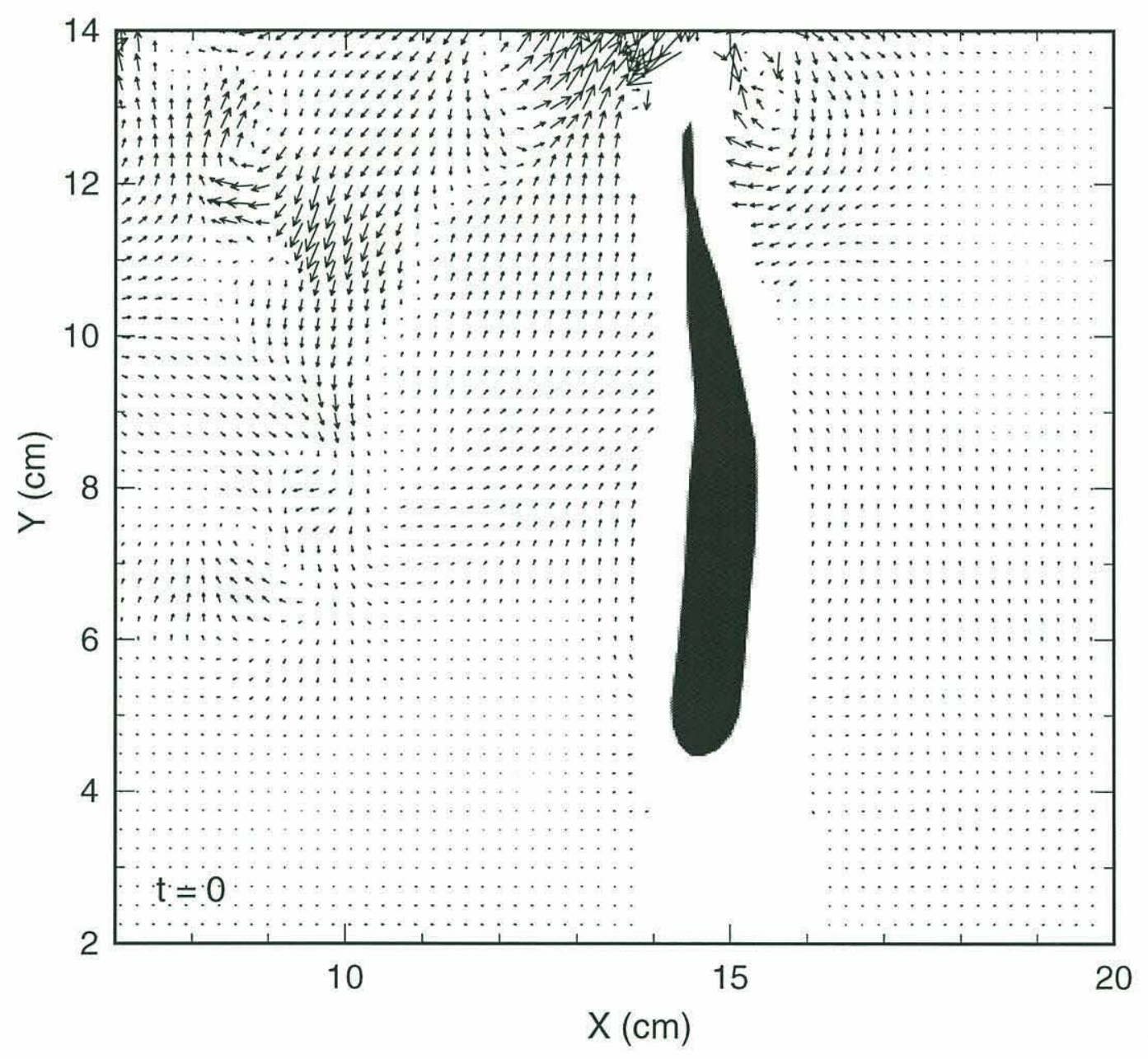

Figure 3-36: Velocity field in the wake of a turning fish, starting position. 


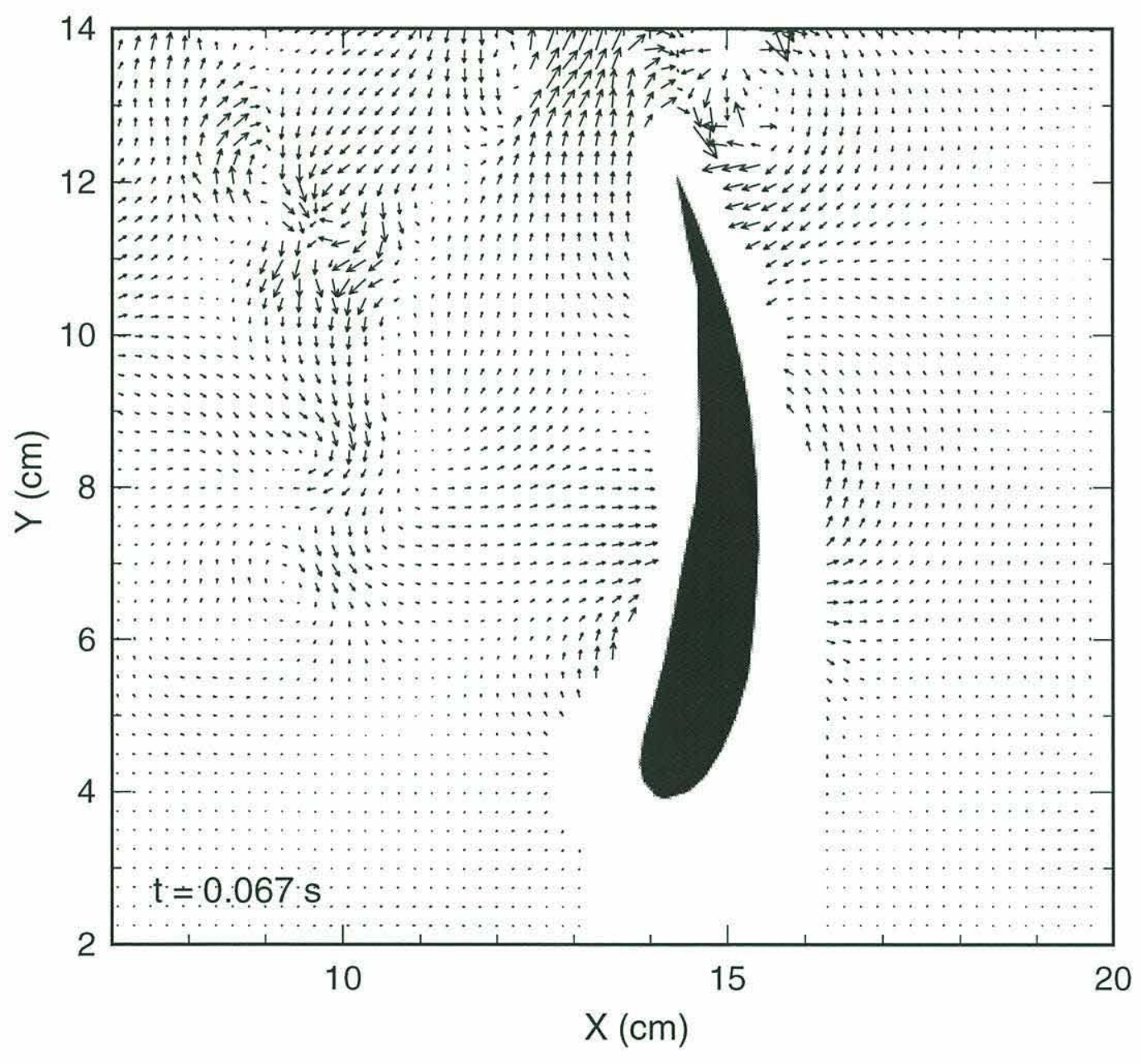

Figure 3-37: Velocity field in the wake of a turning fish. 


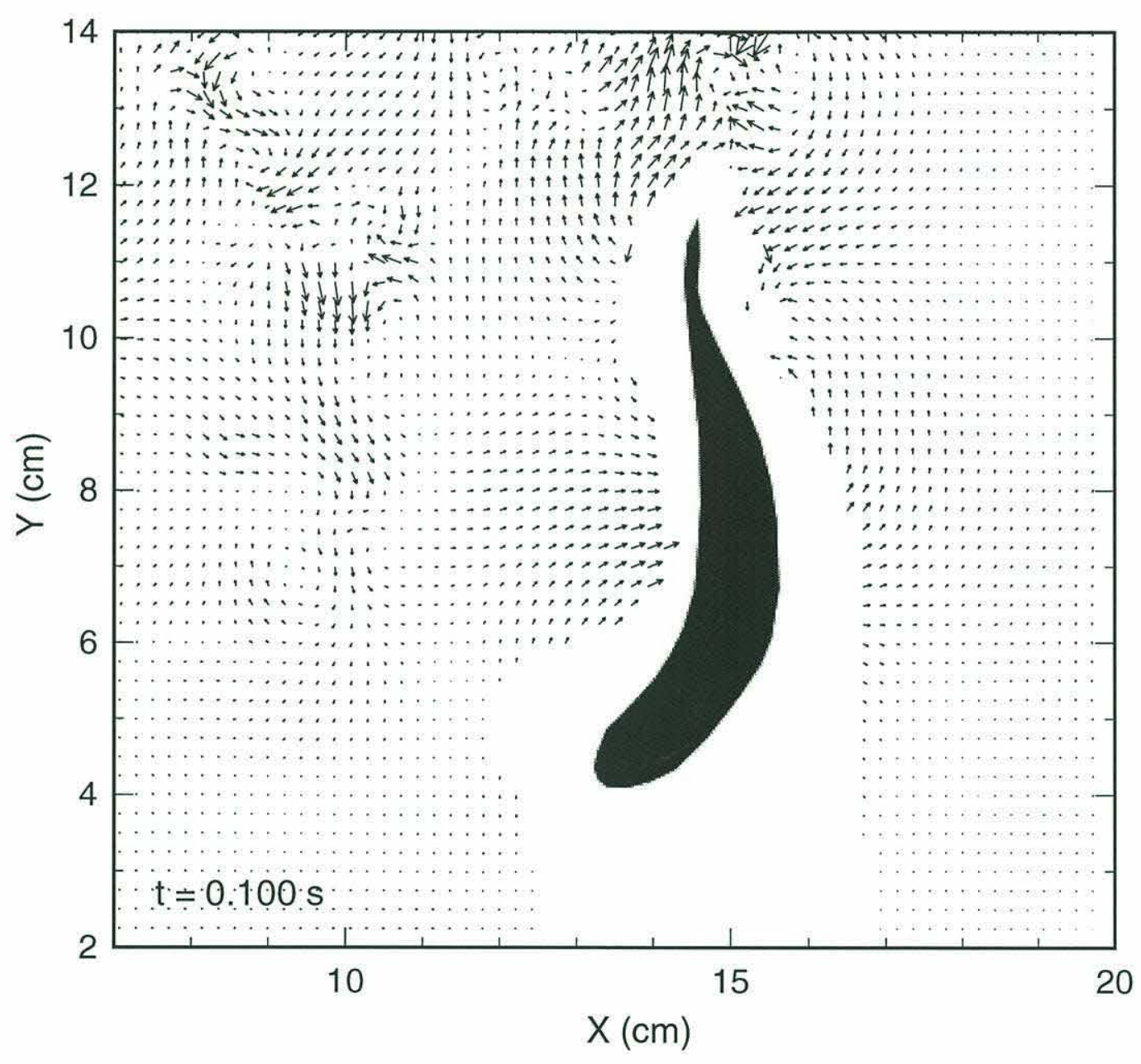

Figure 3-38: Velocity field in the wake of a turning fish. 


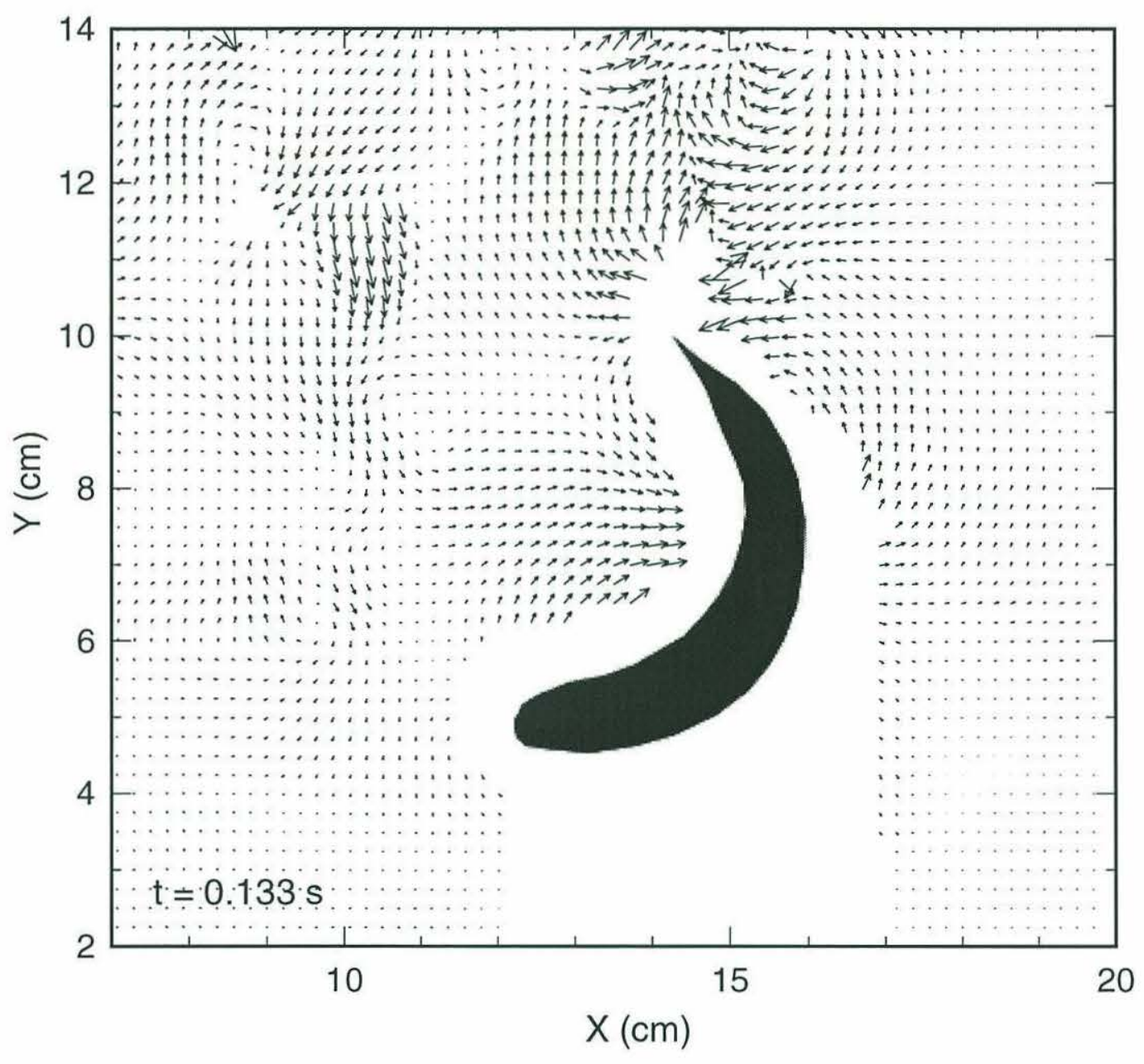

Figure 3-39: Velocity field in the wake of a turning fish. 


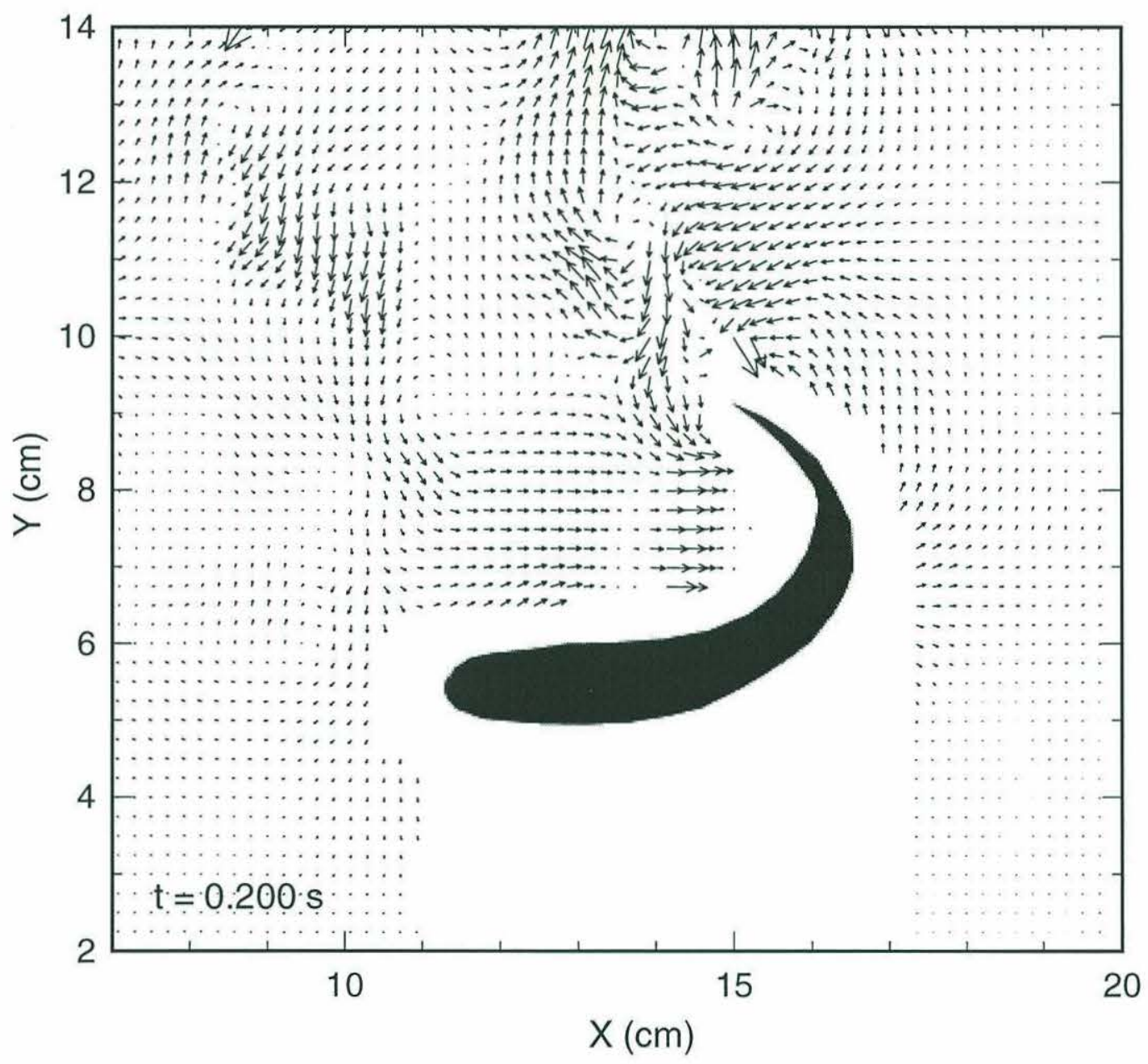

Figure 3-40: Velocity field in the wake of a turning fish. 


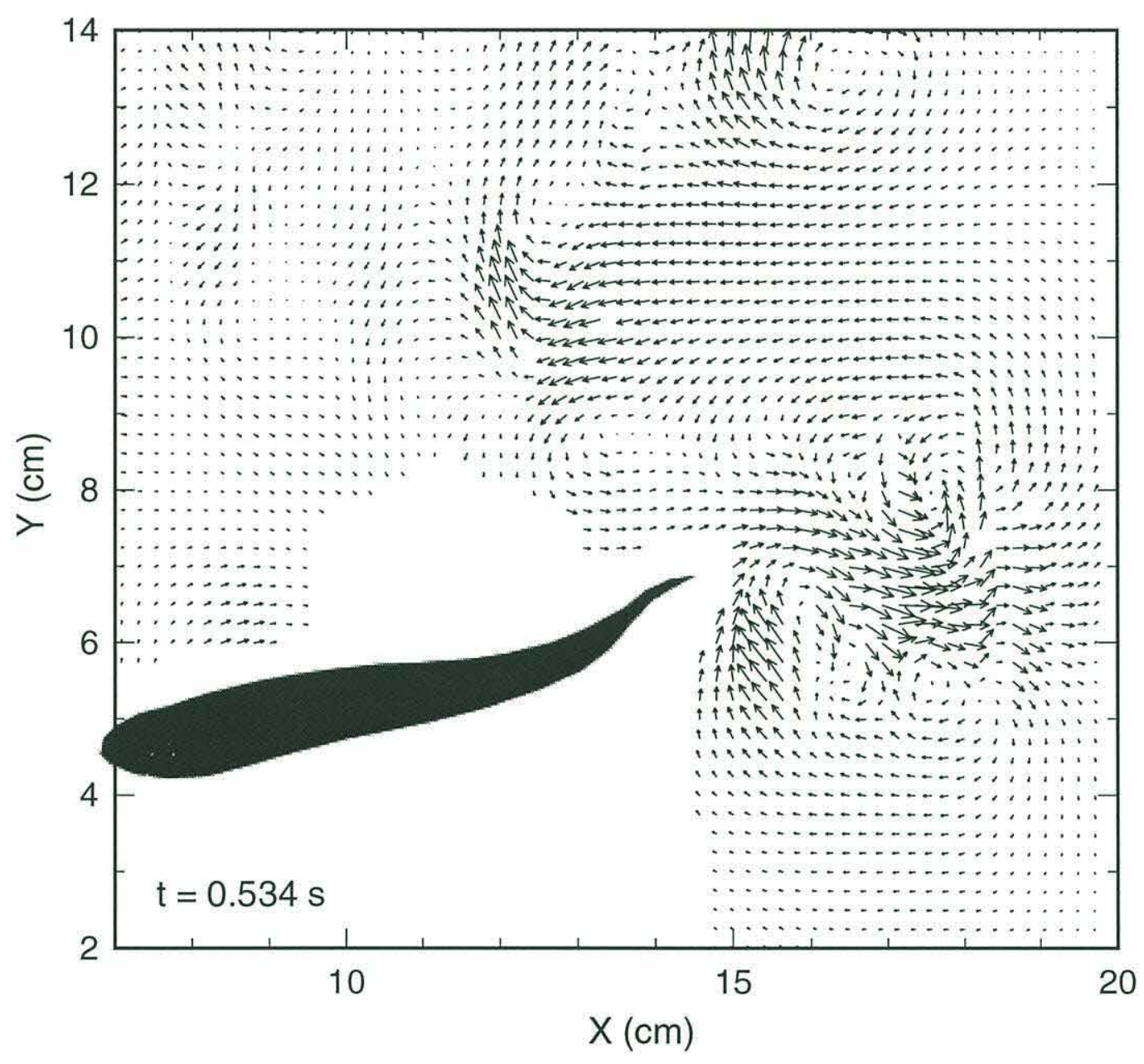

Figure 3-41: Velocity field in the wake of a turning fish. 


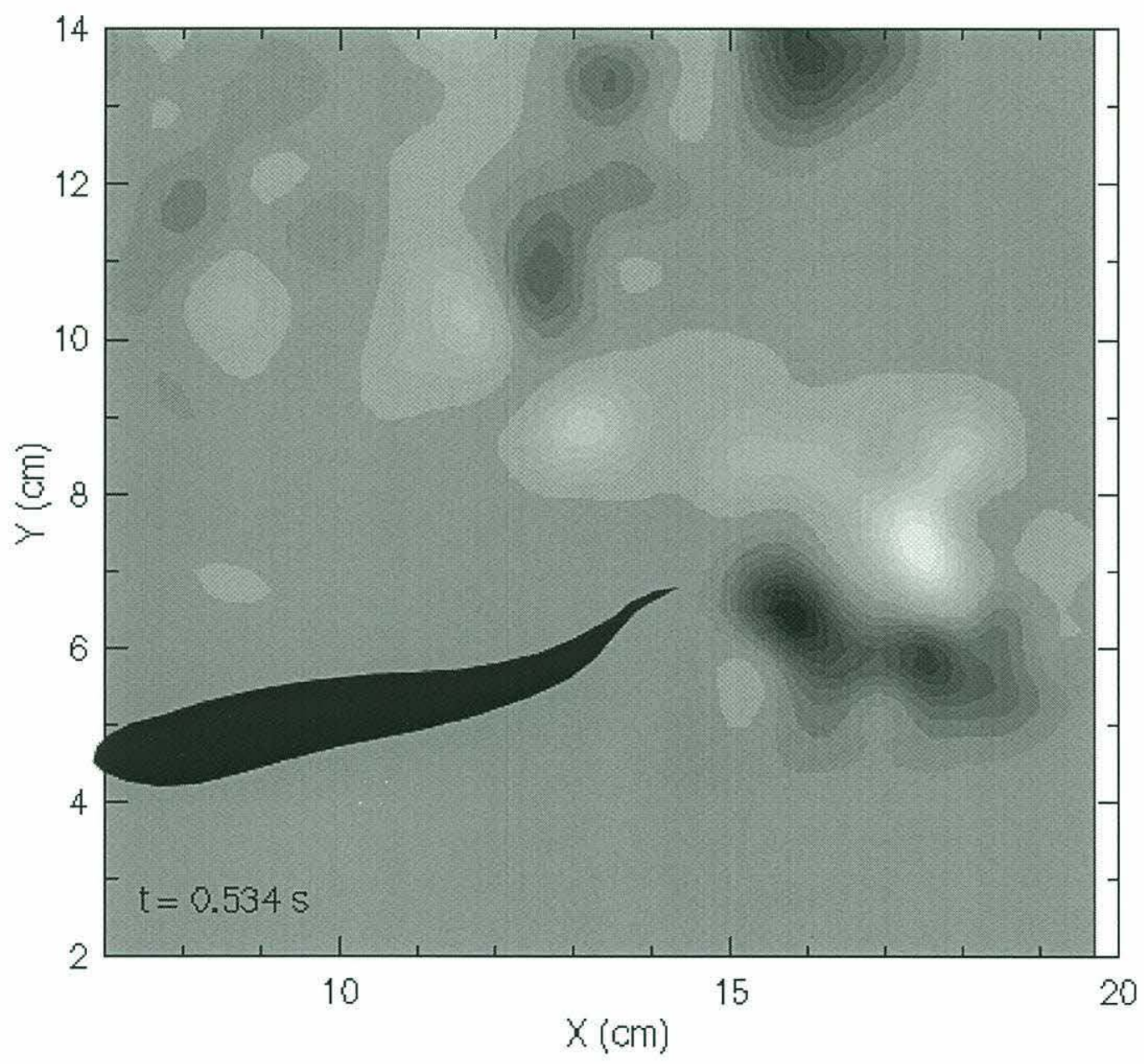

Figure 3-42: Vorticity field in the wake of a turning fish. Contours are from -20.0 (black) to $20.0 \mathrm{~s}^{-1}$ (white) in increments of $1.0 \mathrm{~s}^{-1}$. 


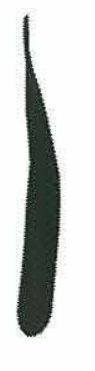

(a)

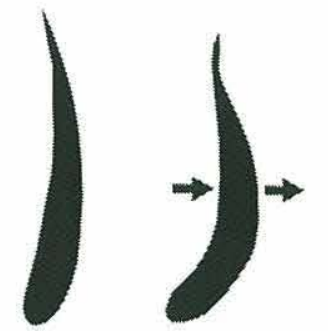

(b)

(c)

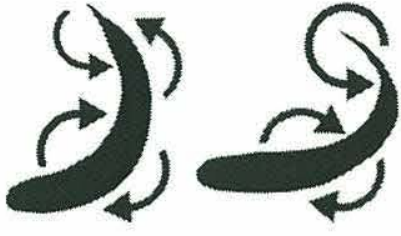

(e)

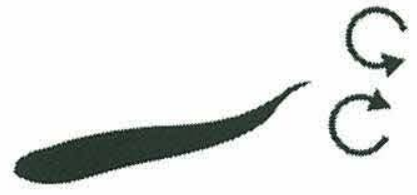

(f)

Figure 3-43: Flow structure around a turning fish. The fish starts the maneuver vertically (a), then bends into a tight curve (b)-(e). The arrows schematically represent body bending and subsequent fluid motion. As the body bends, the rotating cell of fluid moves posteriorly. In (e), it is poised to be reinforced with the tail motion. In (f), the fish resumes straight swimming, leaving a two vortex jet wake.

\subsection{The vertical plane: Three-dimensional effects}

The vertical plane perpendicular to both the fish body axis and the direction of motion was studied to ascertain the flow in the third dimension. Lighthill's [64] elongated-body theory predicts that most of the fluid motion relevant to propulsion lies in this plane. Using the deep water apparatus, the DPIV image plane was oriented vertically along the short axis of the tank near the middle of the tank. The fish was allowed to swim freely in deep water and when the fish pierced the plane, the flow was revealed.

It was difficult to confine the fish to swim through the light sheet at a prescribed time and phase in the swimming cycle. Also, the fish tended to turn sharply when first entering the laser sheet perhaps due to distress when the light sheet was directly on the eyes. Hence, we acquired several excellent vertical plane images for turning maneuvers but few successful passes for normal straight swimming.

Our observations and conclusions in the transverse (vertical) plane are best described qualitatively. During normal straight swimming and coasting through the DPIV image plane, little fluid motion in the vertical plane was observed. Tip vortices shed from extended pectoral fins and the tail during swimming motions were clearly visible. However, significant flow in the elongated body theory sense was not observed for normal straight swimming.

However, when the fish turns abruptly, separated flow in the peduncular region is clearly visible. As the peduncle begins to recoil during the main propulsive stroke, vortex sheets 
shed from both the dorsal and ventral edges of the fish. These vortex sheets are of the same sign such that they connect in the correct sense to the bound vorticity present in the bent body. This indicates that the vortex pair that makes up the jet wake is actually a vortex ring formed by the vigorous body movements. Peduncular shedding contradicts the assumption by Weihs [131] based on kinematic observations that the angle of attack is low during the main propulsive stroke. Shedding over the peduncular region is a mechanism for transferring momentum quickly to the wake in the form of a single vortex ring.

\subsection{Conclusions and recommendations for further research}

In this chapter, we have fulfilled the dreams of many hydrodynamicists fascinated by the propulsive capabilities of swimming fish. Using DPIV, we made quantitative measurements of the flow around straight swimming and maneuvering fish. As expected, the wake arranges into a reverse Kármán vortex street jet. The vortices persist in the wake for at least one fish length behind the tail. The undulation of the fish body significantly affects the development of the wake vortices, beginning at approximately the fish mid-body. The propulsive wave travelling along the body and the associated bound vortices induce the flow near the body to organize into cells destined to become vortices upon arrival at the tail. The formation of a staggered array of alternating vortices at the optimal Strouhal frequency appears to be a fundamental mechanism in the formation of thrust in straight swimming fish.

The body-wave motion has significantly more effect during turning maneuvers. Dramatic bending of the body and accompanying fluid motion are the basis for the formation of two vortices arranged in a jet. Measurements in the vertical plane revealed that during turning, these vortices are actually part of a vortex ring. Contrary to the accepted notion that in turning the body maintains a low angle of attack, dorso-ventral separation in the peduncular region was found to be significant in vigorous maneuvers.

Clearly, there are many improvements that can be made to the experiments executed in this chapter. The most significant difficulty was encouraging the fish to swim straight, away from the laser light source and in the view of the camera. A significant improvement to the method would be to place the fish in a water tunnel to utilize fishes natural rheotropism, that is, the alignment of swimming direction with the flow. By confining the fish in such a tunnel, a variety of speeds and both horizontal and vertical planes could be studied 
systematically. This technique has already been applied in fish physiology studies by many authors, recently by Dewar and Graham [27] with tunas and Rome [95] with scup.

In addition, much exciting research remains to be done in understanding the relationship between morphological features and the body-wave effect. For instance, tunas have many tiny hinged finlets in the caudal peduncle area which certainly affect the flow in this region. It would be interesting to study features such as these and how they relate to propulsion and maneuverability.

Also, time and patience did not permit further study of fast-start and maneuvering hydrodynamics. In future experiments, external stimulus of the fish to turn would significantly improve the experimental method. We feel that we have only scratched the surface of the processes of starting and turning. Much exciting research remains to be done using currently available multipoint flow measurement techniques. It would be beneficial for engineers to work closely with biologists who are expert in the handling and behavior of their subjects. 


\section{Chapter 4}

\section{Experiments in flapping foil propulsion}

\subsection{Introduction}

In this chapter, we use DPIV to experimentally study harmonically oscillated, translating foils. Our goal is to investigate the wake structure and evolution as a function of the foil kinematics. Little is known about the unsteady effects associated with oscillating foils with regards to thrust efficiency and wake structure. The structure of the shed vorticity and the stability of the shear layer are fundamentally linked to the foil motion. Hence, a systematic study of the phenomenon is a substantial undertaking.

Many researchers in the field of aeronautics have looked at translating oscillating foil problems in pure heaving motion (plunge), pure pitching and combinations of both motions. The motivations for unsteady foil research are numerous:

- Aircraft performance in plunge and pitching maneuvers, flutter and stability, basic understanding of oscillatory wakes

- Dynamic stall inception prediction and control for application on high performance aircraft, i.e., supermaneuverability

- Blade-vortex interaction (BVI) for noise prevention in rotorcraft

- Interaction and decay of vortex wakes to minimize hazards to aircraft

- Understanding propulsion of birds, insects and fish

- Propulsion for ships, energy recovery from waves or other unsteady inflow

- Study of separation and unsteady Kutta condition 


\subsection{Background}

Flapping foil propulsion has attracted the interest of many researchers in the field of aerodynamics. Early studies concentrated on understanding flutter and stability for use on aircraft. In 1950, Bratt [17] visualized the wake of a sinusoidally pitching NACA 0015 airfoil with smoke in a wind tunnel up to a chord Reynolds number of $5 \times 10^{6}$. He observed that the wake has a tendency to organize into patches of discrete vorticity and that the wake structure depends on the oscillation frequency. Although Bratt's experiments were limited to small amplitude motions, he observed a variety of wake modes. At low frequencies, the motion generates a turbulent shear layer that follows the trajectory of the trailing edge. As the frequency is increased, the shear layer organizes into four vortices shed per cycle and then two vortices per cycle in a jet arrangement. Clearly, the wake organizes itself in relation to the foil kinematics.

In the literature, the convention has been to present data with respect to the reduced frequency $k=\frac{\pi f c}{U}=\frac{\omega c}{2 U}$ where $f$ is the oscillation frequency in Hertz, $c$ is the chord length, $\omega$ is the frequency in radians per second and $U$ is the free stream velocity. The reduced frequency is a measure of the residence time of a particle convecting over the foil chord compared to the period of the motion. Katz and Weihs [54] show with an inviscid numerical analysis of low amplitude heaving and pitching, that a critical reduced frequency exists, $k=2$, above which the wake rolls up into discrete vortices. Katz and Weihs' method is simplified in that only very low amplitude motions are allowed such that the angle of attack is in the linear part of the lift curve slope. Certainly, wake structure must also depend on other kinematic variables: the amplitude of heave, the amplitude of pitch angle, the angle of attack and the phasing of the motion.

Alternatively, Triantafyllou et al. [120] demonstrate that the governing parameter for flapping foil wakes is not the reduced frequency, but rather, the wake Strouhal number, $S t=\frac{f h}{U}$ where $h$ is the wake width. Triantafyllou et al. show that force coefficient data from Koochesfahani [57] and Isshiki and Murakami [51] collapse onto a single curve with their own data when plotted against Strouhal number. Hence, data from the literature will be converted and referred to in terms of the Strouhal number, rather than the reduced frequency. Since the wake width is not generally known, the trailing edge excursion is substituted for $h$. 


\subsubsection{Visualizations}

Oshima and Natsume [85] and Oshima and Oshima [86] studied heaving and pitching of a NACA 0012 airfoil in a water tunnel at chord Reynolds number 400 to 1200 and 16,000. Flow visualizations and velocity measurements were made and compared to simulation using a discrete vortex method. A wide range of frequencies and large amplitudes of motion of $\mathcal{O}(c)$ were studied.

For pure heaving motions, distinct vortices form for Strouhal numbers greater than 0.08. At low frequencies, the wake resembles a Kármán street along the path of the trailing edge. As the frequency increases, the staggered array of vortices collapses into a straight line, producing a formation where neither drag nor thrust is generated. At larger Strouhal numbers, the wake arranges into a reverse Kármán street, producing a net jet. The angles of attack encountered in these heaving motions are well beyond the steady stall values, typically 40 to 50 degrees. Consequently, a piston type mode of both leading and trailing edge separation is evident at the higher frequencies. Unfortunately, Oshima et al. present little quantitative data to complement their visualizations.

Koochesfahani [57] studied pitching of a NACA 0012 airfoil at small amplitudes around zero angle of attack in a water tunnel. Visualizations and wake velocity measurements were made over a variety of frequencies at at chord Reynolds number of 12,000 .

Koochesfahani's visualizations illustrate the myriad of wake structures possible with simple motions. At low frequencies, a Kármán street is left along the trajectory of the trailing edge. As the frequency is increased, distinct patterns emerge. At $S t=0.10$ based on trailing edge excursion, four vortices per cycle are shed, two of the same sign per half cycle. The structure is remarkably stable for more than 30 chord lengths downstream. Bratt [17] observed the same mode at $S t=0.04$. The difference in frequency is likely due to different pivot points for each. Koochesfahani pitched about the quarter chord point

whereas Bratt's foil was pivoted at the leading edge. At $S t=0.11$ the wake switches to two vortices per cycle arranged in a reverse Kármán street jet formation. As the frequency is increased to $S t=0.14$, the velocity excess in the jet profile increases without apparent increase in jet width.

Koochesfahani's work also demonstrates the strong relationship between wake structure and kinematics of the foil. Pitch waveforms that differ from pure sinusoidal motion create 
asymmetric wake patterns with multiple vortices shed per half cycle. Fast parts of the cycle create a single strong vortex whereas slow parts of the cycle generate two weaker vortices of the same sign. This results in complex wake structures which are not efficient jets. For this reason, we will limit our study to symmetric motions of the foil.

Freymuth [32] studied the combined motions of heave and pitch of a NACA 0015 airfoil in a wind tunnel at chord Reynolds numbers from 5200 to 12,000 . Distinct vortical jet wake patterns were observed in agreement with Oshima et al. $[85,86]$ for $S t=0.34$ and $\frac{2 A}{c}=0.4$. In pure pitch motion about the quarter chord point, a strong jet formed when the maximum angle of attack was around $20^{\circ}$. Weak leading edge separation was observed to merge with and reinforce trailing edge vorticity. At low frequency, the wake was "sluggish" to roll up and was overwhelmed by the drag of the airfoil.

In a later work, Freymuth [33] studied hover modes of insects by considering combined harmonic plunging and pitching motions of a foil in still air. Three different hovering modes were considered: "water treading" with zero average angle of attack, "degenerate figure 8 mode" with $90^{\circ}$ average angle of attack and an oblique mode with fixed angle of attack. The heave amplitude in all cases was $\mathcal{O}(c)$ which produced strong jet vortex wakes with large thrust coefficients. Leading edge separation was observed to be a dominant factor in the wake development. Severing of the stall vortex and subsequent amalgamation with trailing edge vorticity appears to be a useful mechanism for generation of high lift in hover modes.

\subsubsection{Dynamic stall research}

In recent decades, much research has been done on the problem of dynamic stall (leading edge separation) of an airfoil. The dynamic stall vortex (DSV) complicates steady aerodynamics by creating very large forces and moments on the foil that can have either detrimental or beneficial effects. McCroskey [69] presents an excellent review of the problem of time dependent vortical wakes with emphasis on dynamic stall.

The revolutionary Kasper wing [24] is one case where leading edge separation is considered beneficial. A pair of trapped leading edge vortices are captured above an airfoil, resulting in very high lift. In this context, Saffman and Sheffield [101] and Huang and Chow [50] examine trapping of a free vortex by various Joukowski airfoils in steady flight. They demonstrate that high lift can be generated by a stationary trapped vortex over the 
leading edge, but that the vortex is unstable, and will be convected downstream once perturbed. However, a trapped free vortex in the vicinity of the trailing edge was found to provide stable lift many times in excess of the static lift. Thick, symmetric airfoils at low angles of attack are the best candidates for this kind of lift enhancement. Several authors have reported extremely high lift coefficients $\left(C_{L}>10\right)$ for stalled wings when the vortex is maintained over the foil (for example, Gursul et al. [45, 44]).

Interest in aircraft supermaneuverability has spawned much investigation into dynamic stall and separation control. Gad-el-Hak and Bushnell [34] and Wu et al. [140] give excellent reviews of the state of the art in separation control and unsteady excitation of vortex lift. Separation on a lifting surface occurs near the point of maximum loading and thus affects the optimum performance. In general, it is desired to postpone separation; however, in some cases such as the above mentioned Kasper foil, it is beneficial to initiate leading edge separation and reattachment.

Reynolds and Carr [92] summarize the dynamic stall process as follows. As the angle of attack increases past the steady stall angle, the flow around the foil remains thin and inviscid. The boundary layer on the suction side of the foil begins to show signs of flow reversal, beginning at the trailing edge and progressing towards the leading edge. Then, when a critical angle is reached, the flow separates, the boundary layer detaches and a very strong vortical flow develops. A vortex forms near the leading edge of the foil, grows and moves along the foil surface, significantly affecting the performance of the foil.

Many studies on the initiation of the dynamic stall vortex have been done on foils oscillating around nonzero mean angles of attack as in the case of airplane wings. McAlister, Carr and McCroskey [68] performed a systematic survey of the dynamic stall of the NACA 0012 airfoil at chord Reynolds number $2.5 \times 10^{6}$. The model was sinusoidally oscillated in pitch about the quarter chord point at $15^{\circ}$ mean incidence and $10^{\circ}$ pitch amplitude. Velocity and pressure were measured to study the growth and shedding of the stall vortex and quantify the forces. At stall, the lift force increases significantly while the pitching moment decreases. When the vortex leaves the body there is sudden loss of lift and peak negative pitching moment occurs. Large hysteresis loops develop in the lift, drag and pitching moment curves as a function of angle of attack. The sequence of events during dynamic stall is 
1. positive surge in lift force

2. negative moment growth

3. maximum drag force

4. maximum leading edge suction

5. suction wave appears

6. negative roll-off in pitching moment

7. maximum lift force

8. maximum negative pitching moment

Dynamic stall differs from steady stall in two important ways [68]. First, the discontinuities in the moment and lift curves as a function of angle of attack occur at two different times, for two different reasons. The second difference is that in dynamic stall, the dividing streamline from the point of zero shear into the wake encloses a narrow boundary-layer-like zone of reversed flow. By comparison, for static stall, the point of separation coincides with the point of zero shear. The severity of dynamic stall depends on when in the foil oscillation cycle the stall occurs. If a separated vortex forms during the foil upstroke, it is fully developed stall. Rather, if it forms only when the foil reaches maximum pitch, it is partially developed stall and is not as severe. The kinematics of the foil govern the DSV development, reattachment and convection processes.

McAlister et al. [68] found that increasing the reduced frequency postponed stall to progressively higher angles of attack. Partially developed stall can be reversed by increasing the frequency. A suction imprint over the surface of the foil marks the passage of a stall vortex and the magnitude of this imprint increases with increasing reduced frequency. This suggests that the increase in lift is related to the strength of the stall vortex which itself is related to the circulation of the foil at the time the vortex is formed.

McCroskey and Pucci [70] further explored the viscous-inviscid interaction in the dynamic stall process on an oscillating airfoil. They conclude that the primary parameter that determines the degree of separation is the maximum angle of attack. Hence, data from nonzero mean angle of attack experiments can be applied to symmetric oscillations about zero angle of attack. They identify four viscous-inviscid interaction regimes: no stall, stall onset, light stall and deep stall. For the NACA 0012 airfoil oscillating at $k=0.10$ about the quarter chord point, stall onset occurs at $\alpha_{\max }=14^{\circ}$, light stall at $15^{\circ}$ and deep stall 
at $20^{\circ}$. The Strouhal number for this motion is 0.01 . Deep dynamic stall is characterized by a large viscous zone over the foil during more than half of the cycle. The stall angle, the reattachment angle and the severity of the hysteresis in the forces and moments largely depend on the reduced frequency and the angle of attack.

Shih et al. [107] recently studied the DSV formation on a NACA 0012 pitching from zero to $30^{\circ}$ at chord Reynolds number 5000. Particle displacement velocimetry was used to measure the vortex formation and evolution at nondimensional pitch rate $\dot{\alpha}=\frac{\omega c}{U}=0.131$. When the angle of attack exceeds $20^{\circ}$, a vortex forms at the leading edge which then convects downstream. The leading edge vortex triggers the development of a counter-rotating vortex at the trailing edge. A comparative numerical study using a discrete vortex random walk scheme confirmed that the lift force on the foil drops off rapidly at the instant the leading edge vortex separates from the foil surface.

Gad-el-Hak and Ho [35] looked at DSV formation on 3-dimensional lifting surfaces such as delta wings and swept wings as well as the NACA 0012 profile. For oscillations similar to those of McCroskey and Pucci [70], the wake structure was shown to depend on the reduced frequency and the phase angle of the leading edge vortex arrival at the trailing edge. There is mutual induction between the separated leading edge vortex and the shed trailing edge vortex. At $k=\frac{\pi f c}{U}=1.0$, the leading edge vortex pairs with the oppositely signed trailing edge vortex generated during the upstroke. Alternatively, at $k=3.0$, the leading edge vortex arrives at the trailing edge to merge with the same signed trailing edge vortex from the downstroke. Clearly, the resultant wake depends on several factors: the strength and location (phasing) of the separated leading edge vortex which in turn, depend on the frequency (pitch rate) and angle of attack.

Gendrich et al. [36] simulated the effect of acceleration on the DSV formation process. They conclude that the leading edge separation process does not depend on pitch acceleration, but rather, the pitch rate and instantaneous angle of attack.

Robinson and Wissler [94] investigated the DSV formation along the span and near the tip of a NACA 0015 pitching from zero to $60^{\circ}$. They observed that near the tip, vortex-vortex interactions inhibit leading edge vortex convection and enhance lift due to localized suction near the leading edge. Secondary leading edge vortices were also observed which depend on the pitching rate. Pressure measurements indicated little dependence on Reynolds number. 
Visbal and Shang [125] numerically studied essentially the same problem just described, a NACA 0015 foil pitching from zero to $60^{\circ}$. The simulation showed two distinct vortical formations on the suction side of the foil: the dynamic stall vortex and the shear layer vortex which forms aft of the DSV. The DSV and the shear layer vortex interact with each other and eventually combine to form a larger vortical structure. This large separated structure then interacts with the trailing edge vortex. The quantitative characteristics of these vortices depend on the pitch rate and pitch axis location. For a fixed pitch axis location, dynamic stall is delayed until higher angles of attack as the pitch rate is increased, in accordance with the results of McAlister et al. [68].

Ohmi et al. $[82,83]$ studied dynamic stall of a NACA 0012 both numerically and experimentally. The average angle of attack was set to $15^{\circ}$ and $30^{\circ}$ and oscillated with amplitude of $7^{\circ}$ and $15^{\circ}$ at chord Reynolds number 3000. The Strouhal numbers varied from 0.02 to 0.52 . Detailed observations for a wide range of parameters showed the evolution and convection of the DSV. The dominant parameter governing the flow was shown to be the reduced frequency defined here as $f^{*}=\frac{f c}{2 U}$. At low frequency $f^{*}=0.1$, the translating flow controls the wake development while the pitch motion produces a secondary effect. In this case, descent of the leading edge restrains leading edge separation whereas ascent contributes to the separation and DSV development. As the reduced frequency increases to $f^{*}=0.5$, the pitch motion starts to dominate vortex shedding because of fluid inertia. In this case, the transverse motion of the two edges controls the shedding process. Here, descent of the foil aids leading edge vortex formation while ascent restrains it.

At very high frequency, $f^{*}=1.0$, a standing DSV forms due to the superposition of multiple leading edge vortices. Interestingly, at high frequency, the patterns in the wake are "dependent on the product of the the reduced frequency and the amplitude rather than on the frequency itself", which is the Strouhal number multiplied by a constant. Changes in the Reynolds number and the pitch axis were shown to only affect the high frequency cases.

The phenomenon of dynamic stall presents us with some interesting possibilities in flapping foil propulsion. The lift enhancement is certainly beneficial, at least temporarily. If a DSV forms and convects along the upstream facing side of the foil, the lift enhancement may greatly increase the thrust. If this vortex is then shed before the DSV side of the foil faces downstream, the lift can be utilized without the severe penalties when the vortex is 
lost. The moment stall and declining pitching moment could potentially be useful as it can be used to drive the flapping foil in the direction of its desired path. For instance, consider a DSV which leaves the top of the foil trailing edge at the peak in its heave trajectory. The suction from the DSV would act to pitch the foil downwards, driving the foil along its trajectory. Potentially, this extraction of energy could improve efficiency if the DSV is optimally positioned. Alternatively, dynamic stall could act to hinder the foil motion and reduce thrust and efficiency. Clearly, dynamic stall is an important consideration.

\subsubsection{Propulsion studies}

Many authors have addressed the problem of optimal foil motion in the context of animal propulsion. Most restrict their analysis to small oscillations with vorticity shed only from the trailing edge. In two-dimensions, Lighthill [63] calculated the energetic quantities for a rigid airfoil, $\mathrm{Wu}[141,142]$ and Siekmann [108, 109] for a flexible foil and Chopra [21] for a large amplitude heaving rigid foil. Ahmadi and Widnall [4] use integral conservation laws and unsteady lifting theory in two and three dimensions to compute the energetics for general transverse oscillations.

In three-dimensions, Chopra [20] and Chopra and Kambe [22] use unsteady lifting theory to analyze the energetics of high aspect ratio, lunate-tail swimming. Recently, Karpouzian, Spedding and Cheng [53] studied the effect of fin shape on propulsion energetics using lifting line theory extended to curved centerline wings. Bose and Lien [15] employ a strip theory which applies the theories of previous authors to study the propulsive characteristics of a fin whale fluke. Cheng et al. [19] use a vortex ring panel method to analyze the motion of swimming 3 -D waving plates. The goal of most of these works is to analytically predict the forces on oscillating lifting surfaces by modelling the physics of the flow near the foil while neglecting the wake. Alternatively, we focus our attention on the wake structure to gain insight on the fundamental mechanisms of vorticity generation and control.

In the context of fish propulsion, Katz and Weihs [55] simulated large amplitude motions of a hydrofoil with chordwise flexibility. Their inviscid model includes the passive flexibility of a massless, uniformly chordwise flexible 2-D foil. They observed that the thrust increases with increasing frequency, amplitude of motion and angle of attack. The best thrust and efficiency occur when the heave motion lags the pitch motion by approximately $90^{\circ}$. Their model does not account for leading edge separation effects or induced effects from shed 
vorticity. The classic Kutta condition was employed without any special consideration for unsteady effects. Their principal conclusion was that the flexibility of the foil reduces the net lift on the foil but improves efficiency because the direction of the lift is improved. Overall, their model predicts efficiency greater than 80 percent over a wide range of Strouhal numbers.

Katz and Weihs [56] further extended their method to study a slender, flexible propulsor. Their investigation is limited to motions which are small compared to the local span of the foil with low angles of attack. Similar to previous results, thrust and efficiency were shown to increase with increasing heave amplitude. If the curvature of the trajectory is increased, that is, the frequency is increased while keeping the heave amplitude constant, the efficiency decreases. The highest thrust coefficients again occur when the heave motion lags the pitch motion by $90^{\circ}$. As before, high efficiencies are predicted for a wide range of motions.

Videv and Doi [124] numerically studied a rigid 2-D hydrofoil pitching about an arbitrary point in space. Unfortunately, most of their results are for very large pivot distances, that is, the pivot point is several chord lengths removed from the foil either upstream or downstream. This results in flapping motions where the heave velocity depends nonlinearly on the pitch angle. They conclude that the optimal pivot point is upstream of the foil which contradicts Lighthill's [64] conclusion that the pivot should be as far aft as possible. Videv and Doi observed strong leading edge separation due to the lack of feathering in the motion. Their model predicts no thrust at Strouhal number 0.45 when the pivot point is at 10 percent of the chord. This result contradicts other models which predict thrust for this motion.

Many other authors deserve mention for their unsteady foil research. Booth [12] measured the velocity and vorticity in the wake of a NACA 0012 pitching to $10^{\circ}$ at Strouhal number 0.13 which produces a staggered array of vortices in a jet pattern. Robinson et al. [93] looked at the control of wake structure behind an oscillating foil with another stationary foil its wake. Booth [13] studied the loading on a 2-D foil in the wake of a vortex generator to understand the blade-vortex interaction problem.

Mook and Dong [76] numerically investigated oscillating foils alone and in the presence of upstream vortical structures. Vorticity panels are used to model the foil and vorticity blobs to represent the wake with special consideration of the unsteady Kutta condition. For a NACA 0012 pitching $10^{\circ}$ about the quarter chord point at Strouhal number 0.23 , the simulation clearly shows discrete vortices rolling up into a reverse Kármán street. In heave 
only at $\frac{2 A}{c}=0.62$, a similar jet wake is observed at Strouhal number 1.7 with no leading edge separation. The angle of attack for this motion is $80^{\circ}$ which demonstrates the inability of their model to show DSV formation and interaction. Despite this limitation, Mook and Dong's results appear to be useful at moderate angles of attack.

\subsubsection{Force and efficiency measurements}

Ultimately, our bottom line consideration is the efficient production of thrust. Surprisingly, there is little work done on oscillating foil thrust measurements. In 1968, Scherer [105] completed a comprehensive survey of the thrusting capability and efficiency of an oscillating NACA 63A015 airfoil section. A wide range of large amplitude combined heave and pitch motions were studied at chord Reynolds numbers up to $8 \times 10^{5}$ and angles of attack up to $19^{\circ}$. The largest heave amplitude was 0.6 chords at Strouhal number 0.13 . For a given heave amplitude and Strouhal number, the force coefficient increases linearly with increasing angle of attack until the wing stalls at approximately $18^{\circ}$. The motion reaches the maximum efficiency of approximately 60 percent at angles of attack from $7^{\circ}$ to $12^{\circ}$ at Strouhal number 0.13. The phase between heave and pitch was varied to demonstrate that the efficiency and thrust both remain near maximum for a wide range of phase angles. Unfortunately, Scherer did not explore higher Strouhal numbers where both thrust and efficiency are dramatically improved.

DeLaurier and Harris [26] measured the forces on a heaving and pitching NACA 0012 airfoil in a wind tunnel at chord Reynolds number 25,000 to 40,000. The heave double

amplitude was set at $\frac{2 A}{c}=1.25$ and the wing was pitched about the mid-chord to $12^{\circ}$, which gives a maximum angle of attack of around $7^{\circ}$. Although the heave amplitude was large in these experiments, the highest Strouhal number achieved was 0.06 (reduced frequencies $k<0.16$ ) which accounts for the very low thrust coefficients measured. The force coefficient was shown to depend linearly on the reduced frequency for a given angle of attack and phase angle between heave and pitch. Higher angles of attack produced greater thrust. The highest levels of thrust for a given motion occur for phase angles from $90^{\circ}$ to $120^{\circ}$. Again, it is unfortunate that higher Strouhal numbers were not studied.

Recently, experiments were done at the Massachusetts Institute of Technology Ocean Engineering Testing Facility on large amplitude flapping foils by my colleague, David Barrett. Force and efficiency measurements were obtained for a variety of large amplitude 


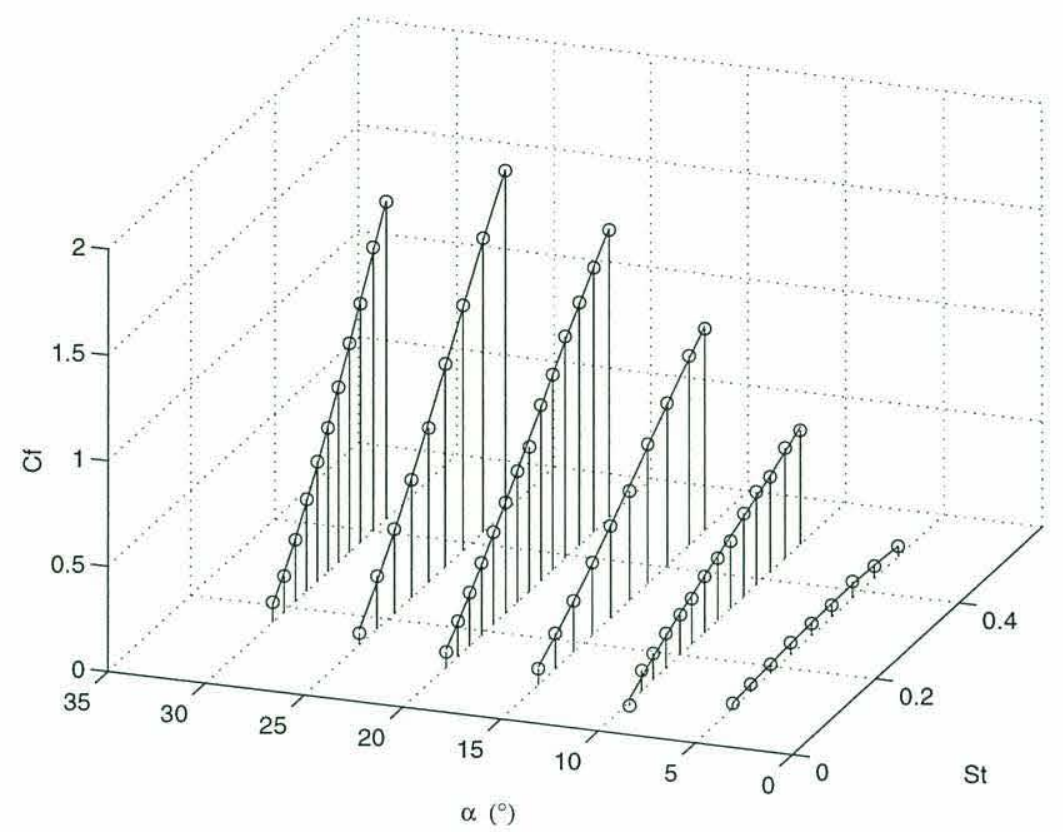

Figure 4-1: Experimental thrust coefficient as a function of angle of attack and Strouhal number for $\frac{2 A}{c}=1.5$ and $R_{c}=4 \times 10^{4}$. Pivot point located $\frac{c}{3}$ from the leading edge. Pitch angle leads heave motion by $\phi=90^{\circ}$.

$\mathcal{O}(c)$ motions up to Strouhal number 0.5. The experimental apparatus and selected data can be found in Triantafyllou et al. [119]. The pitch point, tow speed, maximum angle of attack, heave amplitude, Strouhal number and phase angle were specified. The required pitch amplitude was then calculated to achieve the desired nominal angle of attack.

The thrust coefficient was observed to increase nearly linearly with increasing angle of attack and Strouhal number. The efficiency was highest (near 85\%) for angles of attack between 10 and $20^{\circ}$. High angle of attack motions $\left(\alpha>20^{\circ}\right)$ have peak efficiency at higher Strouhal numbers $(0.3<S t<0.45)$ whereas low angle of attack motions reach peak efficiency at lower Strouhal numbers $(S t \approx 0.1)$ and have declining efficiency for greater Strouhal numbers. Cases with angle of attack between $15^{\circ}$ and $20^{\circ}$ have constant high efficiency for Strouhal numbers between 0.2 and 0.4. All motions have declining efficiency for $S t>0.5$, most likely due to inertial effects related to the rotation of fluid near the foil. Figure 4-1 and Figure 4-2 show the variation in force coefficient and efficiency for the largest amplitude studied.

A modest improvement in efficiency was observed for changes in the phasing between 


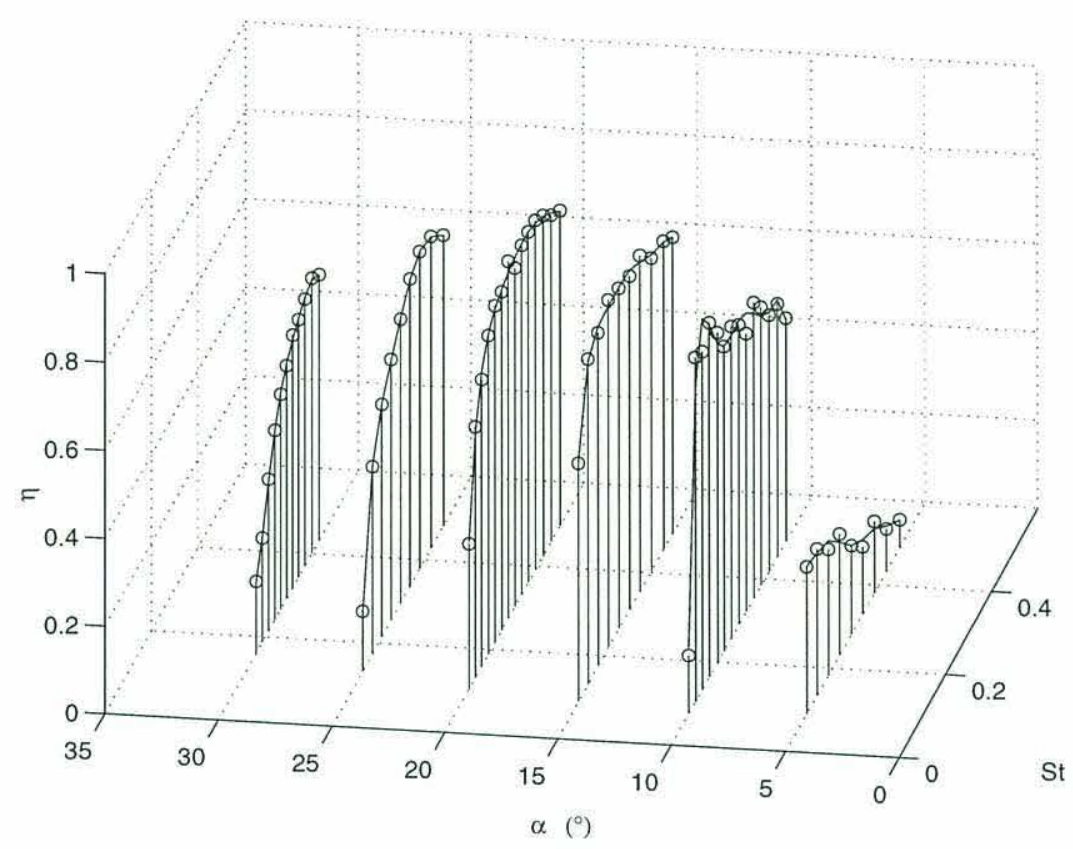

Figure 4-2: Experimental efficiency as a function of angle of attack and Strouhal number for $\frac{2 A}{c}=1.5$ and $R_{c}=4 \times 10^{4}$

heave and pitch. For the majority of the experiments, the pitch angle led the heaving motion by $90^{\circ}$. When the phase angle was lowered to $75^{\circ}$, there was a gain in efficiency of approximately 5 percent in the $20^{\circ}$ angle of attack case while the thrust was reduced only slightly.

Knut Streitlien of the Levich Institute at the City College of New York has simulated large amplitude flapping foils using a 2-D inviscid formulation which accounts for wake selfinduced dynamics $[112,113,119]$. The M.I.T. experimental data are compared to Streitlien's nonlinear simulation in Triantafyllou et al. [119]. At low angle of attack $\left(\alpha=5^{\circ}\right)$ and high amplitude heave motion $\left(\frac{2 A}{c}=1.5\right)$, considerable discrepancy exists between the simulated and experimental thrust coefficient. Simulation and linear theory both overpredict the thrust coefficient and efficiency, especially at high Strouhal numbers. At moderate angle of attack $\left(\alpha=15^{\circ}\right)$ and low heave amplitude $\left(\frac{2 A}{c}=0.5\right)$, simulation and linear theory agree well with experimental results up to Strouhal number 0.3, after which the theory and simulation again both overpredict thrust coefficient and efficiency. At the same angle of attack and large heave amplitude $\left(\frac{2 A}{c}=1.5\right)$, there is excellent agreement between the nonlinear simulation and experiment at low Strouhal numbers. At higher Strouhal numbers 
$(S t>0.3)$, the simulation slightly underpredicts the experimental results in both thrust and efficiency. Linear theory consistently overpredicts thrust and efficiency at high Strouhal numbers.

The goal of this chapter is to further our understanding of the mechanisms which affect thrust and efficiency by focussing our attention on wake structure and evolution.

\subsection{Formulation and definitions}

The heave motion of the foil is referenced to the foil pivot point and is given by

$$
y(t)=A \sin (2 \pi f t)
$$

where $A$ is the amplitude of motion and $f$ is the frequency in Hertz. The pitch angle of the foil leads the heaving motion by phase angle $\phi$ and is given by

$$
\theta(t)=\theta_{0} \sin (2 \pi f t+\phi)
$$

The instantaneous angle of attack at the pivot point $\alpha(t)$ is determined by the towing speed, the heave velocity and the pitch angle. Figure $4-3$ shows the relationship graphically. It is given by

$$
\alpha(t)=\theta(t)-\tan ^{-1}\left(\frac{\dot{y}(t)}{U}\right)
$$

Figure 4-4 spatially illustrates the kinematics of the flapping foil when $\phi=90^{\circ}$. The pitch angle achieves its maxima when the foil pivot crosses the centerline. Usually, the angle of attack will also have its maxima at the centerline crossing, $180^{\circ}$ out of phase with the pitch angle.

When the phase angle $\phi$ is $90^{\circ}$, the maximum angle of attack is given by

$$
\alpha_{\max }=\left|\theta_{0}-\tan ^{-1}\left(\frac{2 A f \pi}{U}\right)\right|=\left|\theta_{0}-\tan ^{-1}\left(\pi S t_{A}\right)\right|
$$

where $S t_{A}$ is the Strouhal number based on the heave amplitude, which is an approximation of the trailing edge amplitude for small pitch angles. The angle of attack phase depends on the phase angle between heave and pitch in addition to the magnitudes of pitch and the wind angle. If $\phi=90^{\circ}$ and if the argument of the absolute value in equation (4.4) is 


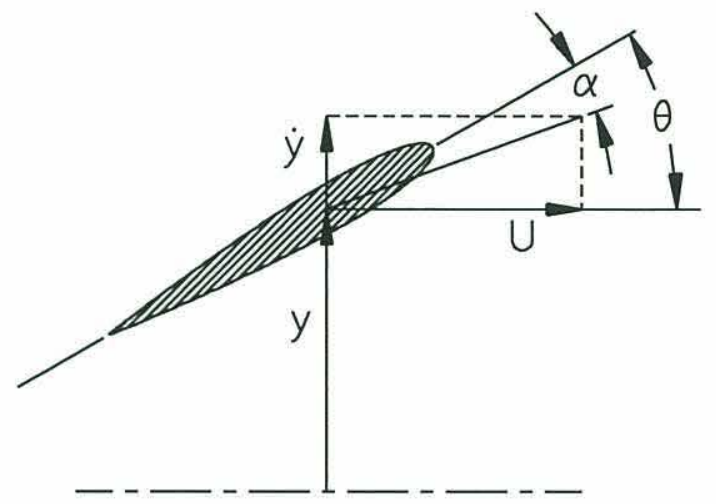

Figure 4-3: Definitions of foil motion. $\theta$ and $\alpha$ are shown as positive.
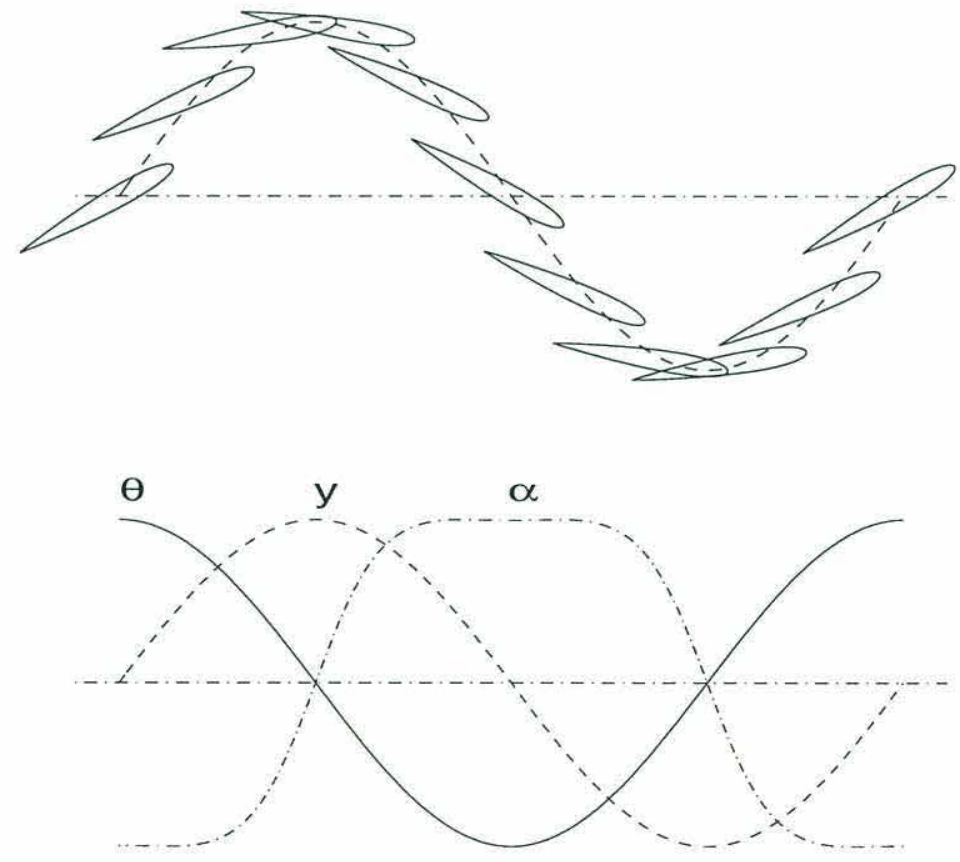

Figure 4-4: Kinematic relationships for flapping motion when $\phi=90^{\circ}$. The heave $y$, pitch angle $\theta$ and angle of attack $\alpha$ are shown normalized with respect to their maxima. 
positive, that is, the pitch amplitude is greater than the angle the "wind" vector makes with the horizontal, the angle of attack is in phase with the pitch oscillation and leads the heave oscillation by $90^{\circ}$. If the argument is negative, the angle of attack oscillation lags the pitch by $180^{\circ}$ and the heave by $90^{\circ}$. When the phase angle differs from $90^{\circ}$, the maximum angle of attack is computed numerically rather than by using equation (4.4) since the peak moves away from the half period points in the oscillation cycle.

The phasing of the angle of attack with respect to the heave and pitch motions determines the direction of the lift force on the foil. Figure 4-5 illustrates this point graphically. In Figure 4-5 (a), the pitch amplitude exceeds the wind angle by $10^{\circ}$, and in (b), the wind angle exceeds the pitch amplitude by $10^{\circ}$. The arrows on the foil indicate a linear steady lift force which is proportional to the instantaneous angle of attack and oriented perpendicular to the wind vector. When the angle of attack is positive during the upstroke and negative during the downstroke, the forward component of the lift force is negative which creates a net drag force on the system. Alternatively, when the sign of the angle of attack is reversed, the net force on the foil will have a positive forward component. In order to create thrust, the foil should move such that the pressure side of the foil faces the heave motion direction.

\subsection{Momentum analysis}

The general form of the momentum theorem for a control volume $\mathcal{V}$ bounded by a control surface $\mathcal{S}$, states that the force vector on the fluid inside the control volume is equal to the material derivative of the fluid momentum integrated over the volume [7]

$$
\vec{F}=\frac{D}{D t} \int_{\mathcal{V}} \rho \vec{v} d \mathcal{V}=\int_{\mathcal{V}} \frac{\partial}{\partial t}(\rho \vec{v}) d \mathcal{V}+\int_{\mathcal{S}} \rho \vec{v} \vec{v} \cdot \hat{n} d \mathcal{S}
$$

where $\hat{n}$ is the local normal vector along the control surface. The first term of the momentum equation is the rate of change of momentum inside the control volume and the second term accounts for the flux of momentum across the control volume boundary. The force vector

$\vec{F}$ encompasses all forces on the control volume including body forces such as gravity and pressure and viscous forces on the surface boundaries.

Consider the control volume $A B C D$ fixed to the foil in Figure 4-6. The boundaries $A B$ and $C D$ are sufficiently far away from the foil in the undisturbed fluid that there is no momentum flux through these surfaces. At the upstream boundary $B C$, the velocity 


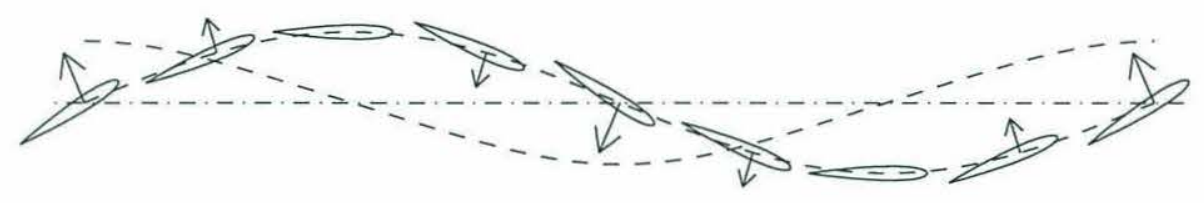

(a)

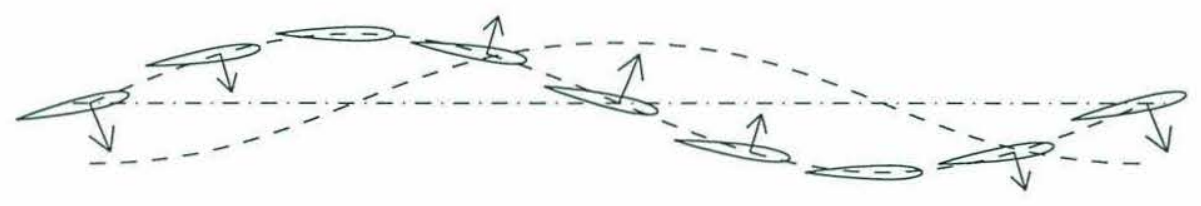

(b)

Figure 4-5: Importance of angle of attack phase. The dashed line indicates the sign and relative magnitude of $\alpha(t)$. The arrows are a linear, steady approximation of the lift vector at each angle of attack. (a) $\theta_{0}$ exceeds $\tan ^{-1}\left(\frac{2 A f \pi}{U}\right)$ by $10^{\circ}$ and forward component of force is negative (b) $\tan ^{-1}\left(\frac{2 A f \pi}{U}\right)$ exceeds $\theta_{o}$ by $10^{\circ}$ and forward component of force is positive. 


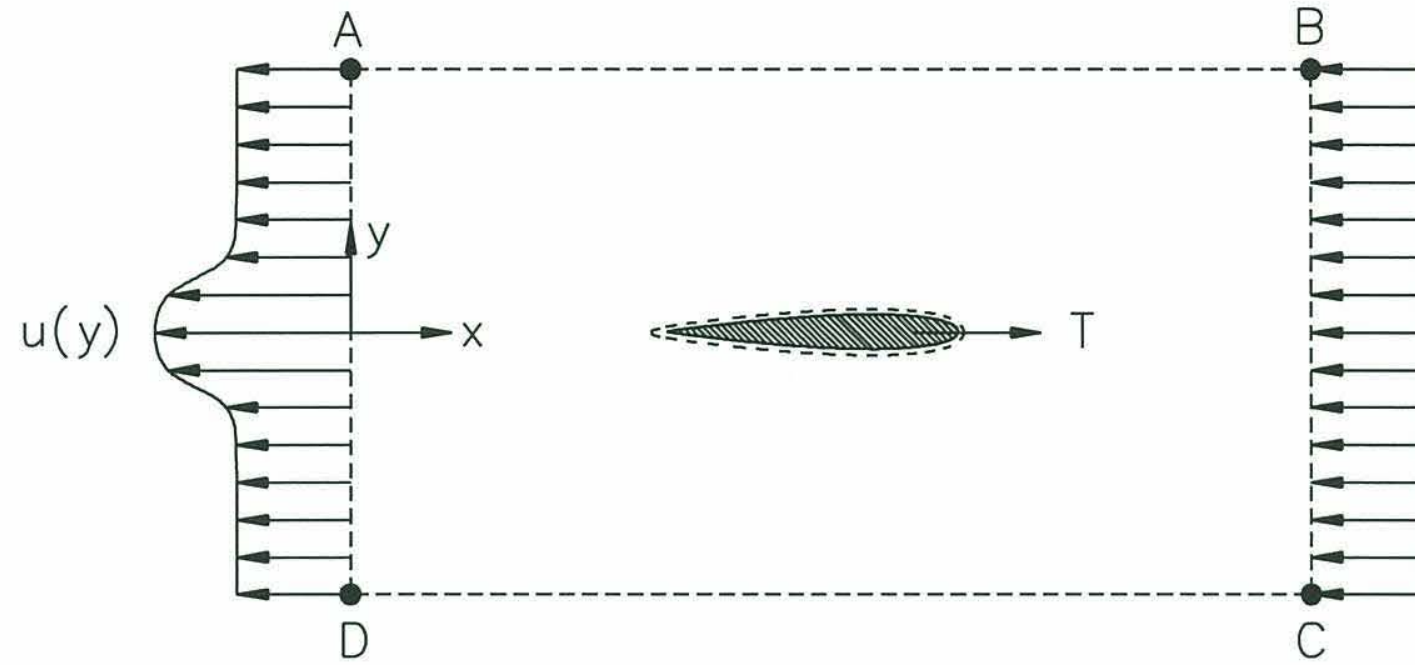

Figure 4-6: Control volume for momentum analysis

across the boundary is the towing velocity $U$. At the downstream position $A D$, the velocity across the boundary is $u(y)$, referenced to inertial coordinates. There is no momentum flux through the foil surface boundary.

If we assume that the average flow is steady and incompressible and that the pressure is constant along the control volume boundaries, that is, the downstream pressure is equalized to the free stream value, the thrust on the foil $T$ can be written in terms of the flux of momentum in the $x$-direction as

$$
T=-F_{x}=\rho \int_{-\infty}^{\infty} U^{2}-u(y)^{2} d y
$$

If we apply mass conservation, then equation (4.6) simplifies to

$$
T=\rho \int_{-\infty}^{\infty} u(y)(u(y)-U) d y
$$

which is exactly the same result obtained by von Kármán for the drag on a flat plate in terms of the momentum defect. This result, although widely used, is based on highly restrictive simplifying assumptions: steady flow, constant pressure, and conservation of mass in two dimensions. In our experiments with flapping foils, we expect to violate all three of these assumptions. Vortex wakes are unsteady, with variable pressure, and axial transport of mass out of the control volume within the vortex cores. 
Although with DPIV we have full field velocity data and can compute the time rate of change of momentum inside the control volume, we do not know the pressure along the control volume boundaries.

An alternative model based momentum method presented by Milne-Thomson [75] utilizes an unsteady momentum analysis to compute the drag of a staggered array of vortices arranged in a Kármán street. The method can be applied to a jet wake by reversing the strength of the vortices. The control volume is defined such that the regime along the boundary is steady: the control volume cuts through the wake in between vortices such that no singularities occur on the contour. Also, the control volume translates at the wake velocity rather than the body velocity to prevent vortices from crossing the control volume wake boundary. Thus, because the pressure along the wake boundary is steady, we can use the irrotational, steady pressure equation. Using point vortex representations to model the wake, the drag force is

$$
D=\frac{2 \pi \kappa \rho b}{a}(U-2 V)+\frac{2 \pi \kappa^{2} \rho}{a}
$$

where $\kappa=\frac{\Gamma}{2 \pi}$ is the strength of the point vortices, $a$ is the streamwise spacing of same side vortices and $b$ is the width of the vortex street. $U$ is the free-stream velocity and $V$ is the vortex convection velocity

$$
V=\frac{\kappa \pi}{a} \tanh \frac{\pi b}{a}
$$

Milne-Thomson's method is difficult to implement in a real vortical wake of limited spatial size. First, in our experiments, the sample size of the wake is small enough that all of the parameters of interest $(\kappa, a, b$, and $V)$ are variable. Also, in many cases it is not possible to design a control volume that does not approach the finite cores of real vortices. Unfortunately, this simple and elegant model does not perform well in our experiments.

Because of the difficulties associated with implementation of the complete unsteady momentum theorem, we chose to use the straightforward and widely used momentum flux calculation of equation (4.6). Comparison with actual force measurements for both flapping foils and oscillating cylinders will show that this approximation is an excellent measure of the force.

We normalize the net thrust on the foil $T$ in the usual way with respect to the dynamic head, chord and span

$$
C_{f}=\frac{T}{\frac{1}{2} \rho U^{2} c s}
$$


where $c$ is the chord and $s$ is the span. Unit span is used in our normalizations since the flow is two-dimensional.

\subsection{The experimental system}

Figure 4-7 shows the tow tank used in the following experiments. The glass tank was constructed specifically for visualizations and DPIV measurements. The dimensions of the internal volume are $0.75 \mathrm{~m}$ wide $\times 0.75 \mathrm{~m}$ deep $\times 2.4 \mathrm{~m}$ long. A motor driven carriage moves along the top of the tank from which various test sections and assemblies can be mounted. The depth of the tank was chosen to give aspect ratios large enough to ensure two-dimensional flow for cylinders and foil sections.

The tow speed of the carriage is set by a controller for the $\mathrm{AC}$ drive motor. For the following experiments, the motor controller was tuned for best performance at the low tow speed studied. The speed was calibrated by timing the carriage over a known distance and was found to be very stable (within 1\%) over time. Very high water quality was maintained during the experiments. The water was reverse-osmosis filtered and chlorine treated to prevent algal growth. Each day, the tank was overfilled and surface skimmed using a few drops of Ivory dishwashing liquid to clean the surface. The surface layer was removed with a standpipe.

An oscillating mechanism was mounted on top of the carriage which simultaneously executes sinusoidal heave and pitch motions on the test section with a single DC motor. The scotch yoke, four bar linkage mechanism was designed and built by my colleague, David Barrett, and is detailed in $[38,39]$. The apparatus allows for multiple tandem sections; however, in this chapter we consider only a single foil section. The amplitude of heave is controlled by setting the excursion of a cam follower coupled to a linear slide. The possible heave settings are continuous from zero heave to $7.62 \mathrm{~cm}$, single side amplitude. The pitch angle amplitude is limited to discrete settings: $0^{\circ}, 7^{\circ}, 15^{\circ}, 30^{\circ}, 45^{\circ}$ and $60^{\circ}$. The phase angle between heave and pitch motions is continuously variable and can be set to any value by rotating the drive wheel with respect to the linkage to the pitch wheel. The frequency of the motion is continuously variable from zero to approximately $0.7 \mathrm{~Hz}$ by adjusting the voltage to the DC motor.

All of the following experiments were done with a NACA 0012 airfoil section with 


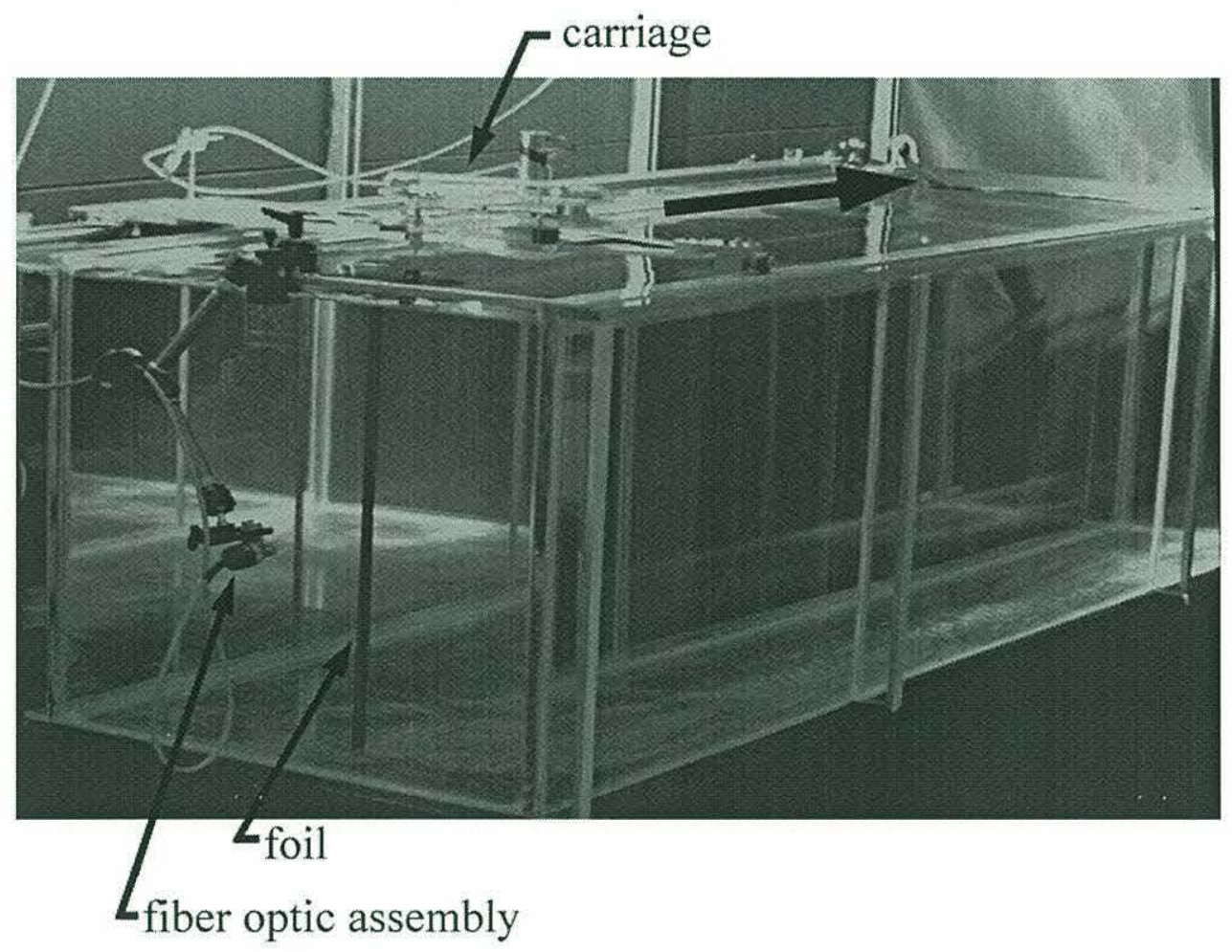

Figure 4-7: Visualization and DPIV tank. The carriage rides along the top of the tank, driven by an AC motor and belt-drive system. Test sections are mounted vertically from the carriage with a free end very near the tank bottom. The fiber optic assembly directs the laser beam through a lens to illuminate a sheet in the middle of the tank volume. The unlabelled arrow indicates the towing direction. 
$3.81 \mathrm{~cm}$ chord length, minimum submerged length of $71 \mathrm{~cm}$ and aspect ratio 18.6. The foil was carefully constructed by casting epoxy resin with added carbon fiber tape and an embedded steel rod. The foil was cast with a precision cut hardwood mold in two halves. First, the molds were partially filled with epoxy, allowed to partially set and then carbon fiber tape was carefully laid into both sides. More epoxy was added to secure the tape, then the rod was laid into one side of the mold with specially designed end caps to hold it in place. After curing to a jelly-like state, more epoxy was added before closing the mold and clamping. The finished product was wet sanded with special treatment given to the trailing edge. The edge was kept very sharp and free of macroscopic defects. The foil was painted black and waxed to a smooth finish.

Digital particle image velocimetry (DPIV) was used to map the flow around and in the wake of the flapping foil. All measurements were made in the horizontal plane perpendicular to the span of the foil at the tank mid depth $(36.2 \mathrm{~cm}$ from the bottom). The video camera was located horizontally below the tank, looking at a mirror oriented at $45^{\circ}$ below a viewport in the tank bottom. This technique avoids the difficulty of looking through the free surface which distorts the optical path. However, this method precludes the use of an endplate on the foil free end. This was not a problem since in the midplane, the flow was two-dimensional with very low divergence.

The foil was started from rest at one end of the tank, approximately $1.1 \mathrm{~m}$ or 29 chord lengths ahead of the viewport. DPIV data were collected in a plane $22 \mathrm{~cm}$ ( 5.8 chords) long in the towing direction and $15 \mathrm{~cm}$ (3.9 chords) in the lateral direction. Some of the data are unusable due to shadowing of the laser sheet in the upstream direction. These data are a small fraction of the flow field and are removed in all presented results. The tank settled for a minimum of twenty minutes between experiments.

Data collection was triggered manually so the phase of the foil motion with respect to the camera field of view varied between runs. The axes on the presented velocity fields are referenced to the camera view (lab reference frame) to avoid distracting coordinate system changes between figures. The laser shutter timing system was set such that the light pulse duration was $15 \mathrm{~ms}$ with an effective time difference between image pairs of $20 \mathrm{~ms}$. 


\subsection{Flapping foil experiments}

Because the possible parameter space for flapping foil motion is so large, we elected to vary selected parameters which have the most effect on wake structure. We consider six parameters which influence the performance of the foil:

- the heave double amplitude to chord ratio $\frac{2 A}{c}$

- the pitch amplitude $\theta_{0}$

- the phase angle $\phi$ between heave and pitch motions

- the foil pivot point $b^{*}=\frac{b}{c}$ measured from the leading edge

- the Strouhal number $S t=\frac{h f}{U}$

- the chord Reynolds number $R_{c}=\frac{U c}{\nu}$

Again, $U$ is the towing speed, $f$ is the frequency in Hertz, $h$ is the wake width which is unknown a priori but can be estimated as the peak to peak foil trailing edge excursion. In our experiments, the foil pivot is held constant at $b^{*}=0.33$ and the Reynolds number is fixed at $R_{c}=1100$. Additionally, we consider the dependent parameters

- the maximum angle of attack $\alpha_{\max }$

- the reduced frequency $k=\frac{\pi f c}{U}$ or $f^{*}=\frac{f c}{2 U}$

- the rate of change of the angle of attack $\Omega^{*}=\frac{\dot{\alpha} c}{2 U}$

- the feathering parameter $\chi=\frac{\theta_{0} U}{2 \pi f A}$

- the path wavelength $\lambda=\frac{U}{f}$

- the path radius of curvature $r_{c}=\frac{\lambda}{2 \pi S t_{A}}$

Ideally, we would like to specify the Strouhal number (wake frequency), the heave amplitude (wake size) and the angle of attack (foil loading) and predict the forces on the foil and the wake structure. It is not possible to specify an arbitrary angle of attack with our oscillation mechanism. The apparatus limits the angle of attack to be a function of the heave amplitude, towing speed, pitch angle and phase angle. Thus, in order to achieve the desired range of angle of attack for a given heave amplitude and Strouhal number, the pitch 
amplitude is adjusted. This presents us with another problem: for specified heave amplitude and Strouhal number, the angle of attack profile during the oscillation cycle is not unique. Multiple angle of attack profiles are possible depending on the pitch amplitude setting. As discussed previously, the wrong selection of kinematic parameters can give angles of attack which do not produce thrust (Figure 4-5).

Figure 4-8 shows three different angle of attack profiles that are possible for the same Strouhal number, heave amplitude and phase angle. The pitch amplitude $\theta_{0}$ determines the angle of attack profile. For low pitch angles, the angle of attack is very large and varies as a smooth sinusoid. As the pitch angle is increased to moderate values, the angle of attack profile flattens into a smooth cornered square wave. At very high pitch amplitudes, the angle of attack is further reduced and oscillates with a multiple peaked profile. The multiple peaked profile causes additional vortices to be shed per period in contrast to two vortices per cycle for the smooth and flat-top sinusoid profiles. The flat-top profile produces greater average thrust compared to the sinusoidal profile because the average angle of attack per half cycle is larger. In addition, the time rate of change of the angle of attack differs between profiles which affects dynamic stall inception and evolution.

Figure 4-9 shows the relationship between maximum angle of attack, pitch angle amplitude and Strouhal number for our apparatus when $\frac{2 A}{c}=2.0$. For all angles of attack and Strouhal numbers, when the pitch angle is less than $15^{\circ}$, the angle of attack profile is a smoothly varying sinusoid which should produce two vortices per cycle when the angle of attack changes sign. When the pitch angle reaches $30^{\circ}$, the $\alpha(t)$ profile flattens for most Strouhal numbers, with degraded performance (multi-peaked $\alpha(t)$ profile or no thrust profile) at Strouhal numbers less than 0.25 . Pitch angles $45^{\circ}$ and above produce only multi-peaked or no thrust profiles.

The curves in Figure 4-9 illustrate our restricted access to the parameter space for our apparatus when $\frac{2 A}{c}=2.0$. Certain regions in the $S t-\alpha_{\max }$ plane are not realizable. Also, in some regions, notably for $S t<0.4$ and $\alpha_{\max }<30^{\circ}$, the angle of attack profile is not uniquely specified by the Strouhal number and angle of attack. In this region, we can expect either no thrust, high thrust, or marginal cases with multiple vortices shed per cycle, depending on the pitch amplitude setting. For lower heave amplitudes, the uppermost curves in Figure 4$9\left(\theta_{0} \leq 15^{\circ}\right)$ are the same as for the $\frac{2 A}{c}=2.0$ case. However, for $\theta_{\circ} \geq 30^{\circ}$, the curves move downward (to lower $\alpha$ ) and to the right (to higher $S t$ ) while keeping the same angle of 

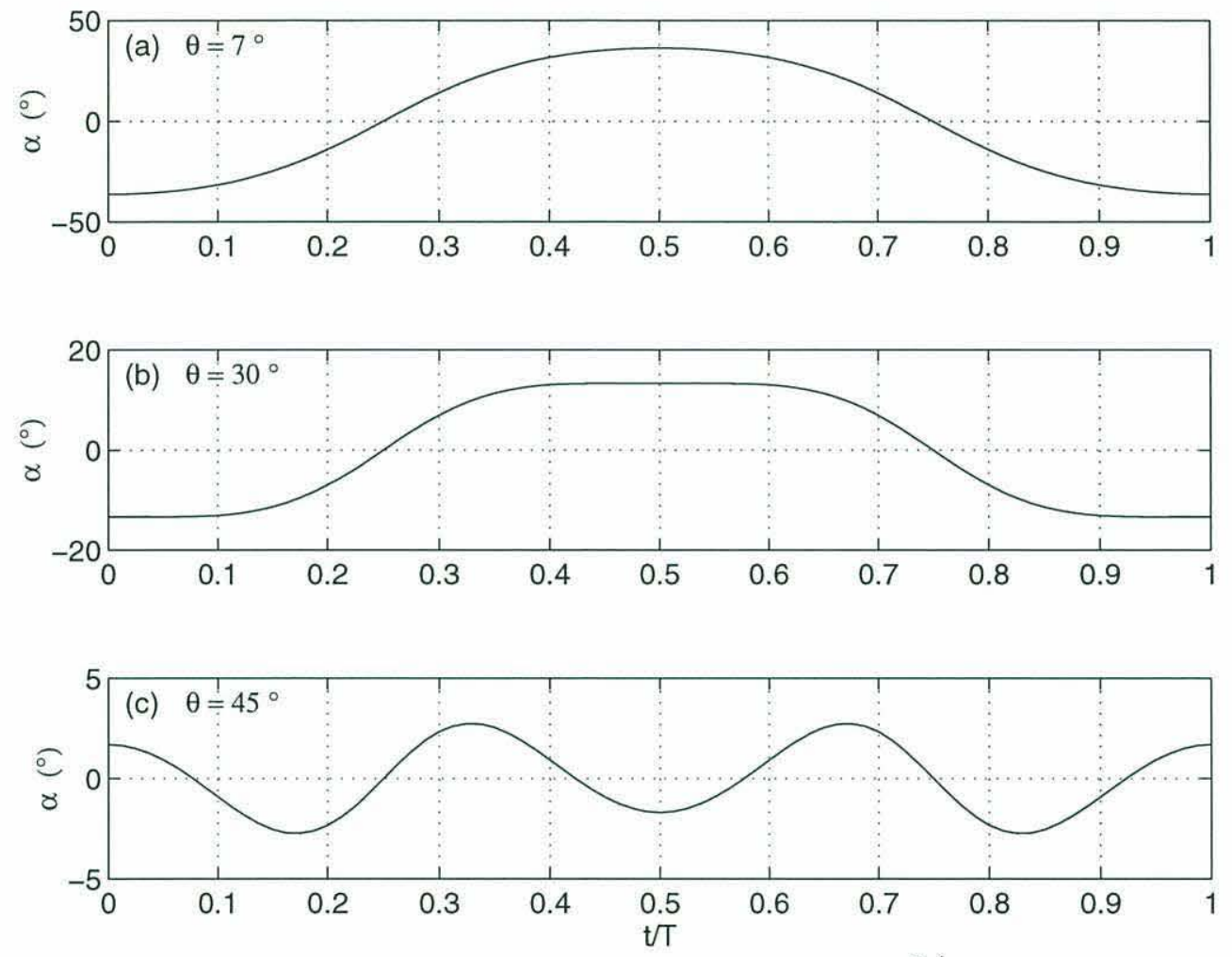

Figure 4-8: Examples of possible angle of attack profiles for $\frac{2 A}{c}=2.0, S t_{A}=0.30$ and $\phi=90^{\circ}$. (a) sinusoid $\alpha(t)$, (b) flattened $\alpha(t)$, (c) multi-peaked $\alpha(t)$. 


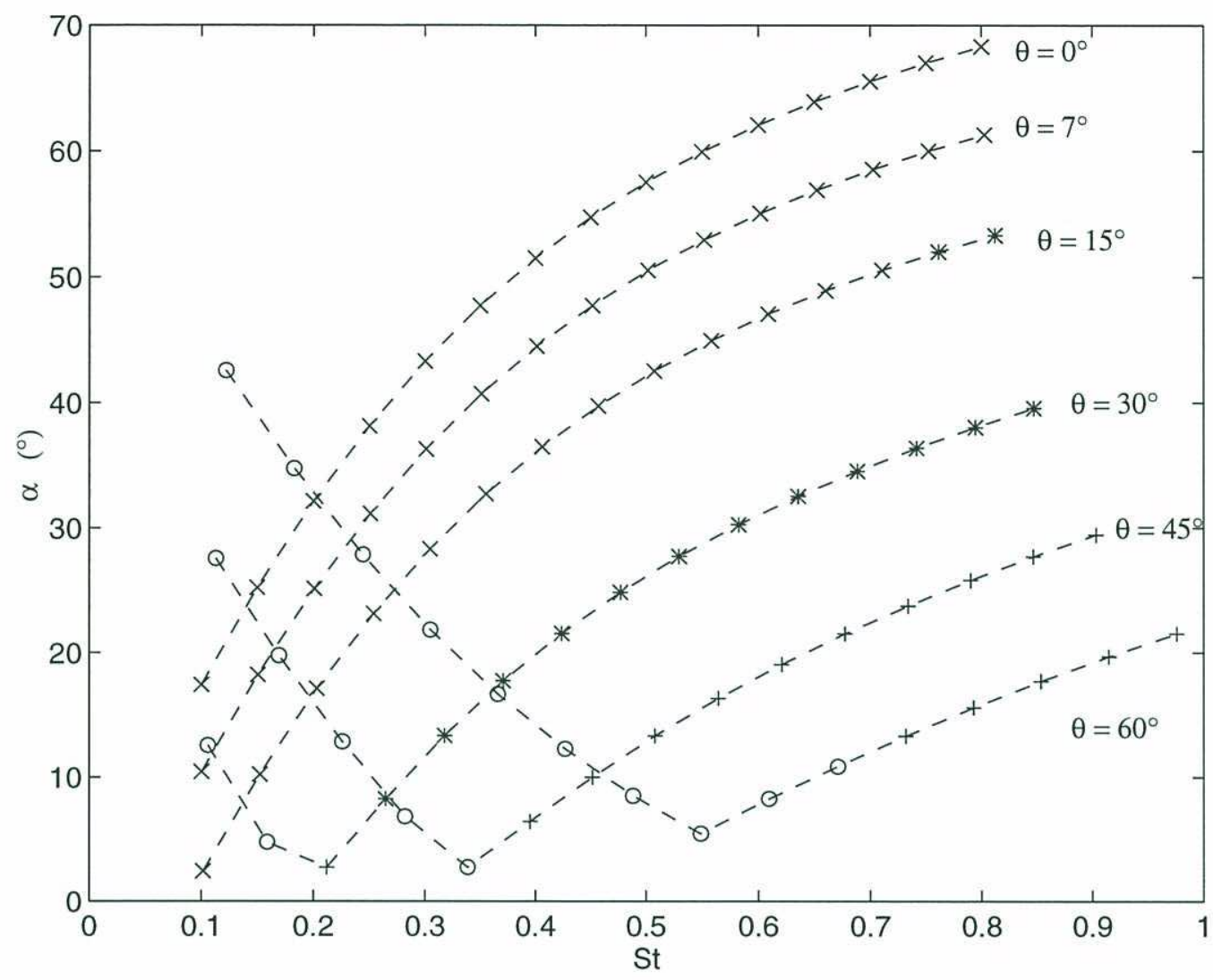

Figure 4-9: Angle of attack profile types as a function of maximum angle of attack, trailing edge Strouhal number and pitch angle amplitude for $\frac{2 A}{c}=2.0$. ' $X$ ' marks indicate normal sinusoidal $\alpha(t)$ profile, '*' marks indicate flattened sinusoidal profile, ' + ' marks indicate double peaked profile and ' $O$ ' marks indicate that no thrust is expected since $\theta_{0}>\tan ^{-1}\left(\frac{2 A \pi f}{U}\right)$.

attack profile. For instance, at $\frac{2 A}{c}=0.5$ and $\theta_{0}=30^{\circ}$, flattened sinusoid $\alpha(t)$ profiles exist at $S t=0.8$ and $\alpha_{\max }=25^{\circ}$ whereas in the large amplitude case the profile is multi-peaked. Hence, we do not expect multiple vortices shed per cycle in low amplitude motions.

Our experimental survey is divided into several small investigations, each intended to show a particular variable's significance to the problem. Table 4.1 summarizes the parameter space and the kinematic properties of the motions. The Strouhal number based on heave double amplitude is varied between 0.1 and 0.45 and the heave amplitude to chord ratio from 0.5 to 2.0 . The reduced frequency range for these motions is sufficiently small $(k<2)$ such that the classic Kutta condition is likely satisfied [90]. The pitch angles were chosen to give large angles of attack, often exceeding the steady stall value. In steady flow at $R_{c}=10^{6}$ the NACA 0012 section stalls at $12^{\circ}$ to $16^{\circ}$ depending on roughness [1]. The 
phase angle was varied between $30^{\circ}$ and $110^{\circ}$ for the motions which produced the largest jet wake, otherwise it was fixed at $90^{\circ}$. The pivot point was fixed at one third of the chord from the leading edge.

First, the effect of Strouhal number was investigated at fixed large heave amplitude and variable angles of attack in groups I through IV. Then, the same Strouhal numbers were tested near one characteristic angle of attack for smaller heave amplitudes in groups $\mathrm{V}$ and VI. Finally, phasing is addressed in groups VII, VIII and IX.

Table 4.2 summarizes the experimental results. The average wake velocity excess in the streamwise direction is computed by averaging several slices of the wake spanning one wavelength behind the trailing edge, at one instant in time. When the wavelength of the motion is larger than the experimental view, two or more snapshots of a portion of the wake are averaged. In all cases, we use whole multiples of the wavelength. $\left|\frac{\overline{u-U}}{U}\right|_{\max }$ is the peak average velocity excess in the inertial reference frame. The accuracy of the averaging technique was verified by comparison with calculations in which the number of slices was doubled or tripled. Further comparison with duplicate runs showed that the average velocity excess is accurate and repeatable to within $2 \%$.

The wake width, $h$, is computed from the lateral excursion of the most recently shed vortex from the centerline of motion, averaged at two points in the oscillation cycle that are $180^{\circ}$ out of phase. More simply, $h$ is the lateral distance between vortex centers in the near wake. $S t_{h}$ is the Strouhal number based on $h$, rather than the heave amplitude or trailing edge excursion. The stability analysis by Triantafyllou et al. [118] demonstrates that $S t_{h}$ is the most meaningful measure of the wake frequency.

We compute the circulation of wake and stall vortices by integrating the velocity along a circular contour around the vortex using equation (2.5). The presented results are for the contour radius which gives maximum circulation, or $R=1.5 \mathrm{~cm}(0.4 c)$, whichever is smaller. Table 4.2 shows normalized values, $\Gamma^{*}=\frac{\Gamma}{U c}$. The $\Gamma_{\text {wake }}^{*}$ values indicate the strength of wake vortices approximately half a wavelength downstream of the trailing edge. $\Gamma_{D S V}^{*}$ is the strength of the stall vortex when the foil is at the maximum heave position.

There are two sources of error that must be considered in the circulation calculation. First, the center of the vortex must be specified accurately. If the center of the integration contour is ten percent of the contour radius in error, the maximum error in the circulation calculation is less than five percent. The second source of error stems from the small age 


\begin{tabular}{|c|c|c|c|c|c|c|c|c|c|c|c|c|}
\hline case & $\begin{array}{l}S t_{A} \\
\frac{2 A f}{U}\end{array}$ & $S t_{T E}$ & $\begin{array}{cc}k & \left(f^{*}\right) \\
\frac{\pi f c}{U} & \left(\frac{f c}{2 U}\right)\end{array}$ & $\frac{2 A}{c}$ & $\begin{array}{l}\theta_{0} \\
\left({ }^{\circ}\right)\end{array}$ & $\begin{array}{c}\phi \\
\left({ }^{\circ}\right)\end{array}$ & $\begin{array}{c}\alpha_{\max } \\
\left({ }^{\circ}\right)\end{array}$ & $\begin{array}{l}\overline{|\alpha|} \\
\left({ }^{\circ}\right)\end{array}$ & $\chi$ & $\frac{r_{c}}{c}$ & $\begin{array}{l}\Omega_{\max }^{*} \\
\left|\frac{\dot{\alpha} c}{2 U}\right|_{\max }\end{array}$ & $\begin{array}{c}\alpha(t) \\
\text { profile }\end{array}$ \\
\hline $\begin{array}{c}\mathrm{I} \\
\mathrm{fa} 04 \\
\mathrm{fa} 05 \\
\mathrm{fa} 06 \\
\end{array}$ & $\begin{array}{l}0.10 \\
0.10 \\
0.10 \\
\end{array}$ & $\begin{array}{l}0.10 \\
0.10 \\
0.10 \\
\end{array}$ & $\begin{array}{l}0.16(0.10) \\
0.16(0.10) \\
0.16(0.10) \\
\end{array}$ & $\begin{array}{l}2.0 \\
2.0 \\
2.0 \\
\end{array}$ & $\begin{array}{c}0 \\
7 \\
15 \\
\end{array}$ & $\begin{array}{l}90 \\
90 \\
90 \\
\end{array}$ & $\begin{array}{l}17.4 \\
10.4 \\
2.4 \\
\end{array}$ & $\begin{array}{l}11.3 \\
6.8 \\
1.7 \\
\end{array}$ & $\begin{array}{c}0 \\
0.39 \\
0.83 \\
\end{array}$ & $\begin{array}{l}31.8 \\
31.8 \\
31.8 \\
\end{array}$ & $\begin{array}{l}0.05 \\
0.03 \\
0.01 \\
\end{array}$ & $\begin{array}{l}\mathrm{s} \\
\mathrm{s} \\
\mathrm{s}\end{array}$ \\
\hline $\begin{array}{c}\text { II } \\
\mathrm{fa} 16 \\
\mathrm{fa} 17 \\
\mathrm{fa} 18\end{array}$ & $\begin{array}{l}0.15 \\
0.15 \\
0.15\end{array}$ & $\begin{array}{l}0.15 \\
0.15 \\
0.15\end{array}$ & $\begin{array}{l}0.24(0.15) \\
0.24(0.15) \\
0.24(0.15)\end{array}$ & $\begin{array}{l}2.0 \\
2.0 \\
2.0\end{array}$ & $\begin{array}{c}0 \\
7 \\
15 \\
\end{array}$ & $\begin{array}{l}90 \\
90 \\
90 \\
\end{array}$ & $\begin{array}{l}25.2 \\
18.2 \\
10.2 \\
\end{array}$ & $\begin{array}{c}16.5 \\
12.0 \\
6.9 \\
\end{array}$ & $\begin{array}{c}0 \\
0.26 \\
0.56 \\
\end{array}$ & $\begin{array}{l}14.2 \\
14.2 \\
14.2 \\
\end{array}$ & $\begin{array}{l}0.11 \\
0.08 \\
0.05 \\
\end{array}$ & $\begin{array}{l}\mathrm{s} \\
\mathrm{s} \\
\mathrm{s}\end{array}$ \\
\hline $\begin{array}{c}\text { III } \\
\mathrm{fa} 01 \\
\mathrm{fa} 02 \\
\mathrm{fa} 03\end{array}$ & $\begin{array}{l}0.30 \\
0.30 \\
0.30\end{array}$ & $\begin{array}{l}0.30 \\
0.30 \\
0.32 \\
\end{array}$ & $\begin{array}{l}0.47(0.30) \\
0.47(0.30) \\
0.47(0.30)\end{array}$ & $\begin{array}{l}2.0 \\
2.0 \\
2.0\end{array}$ & $\begin{array}{c}7 \\
15 \\
30 \\
\end{array}$ & $\begin{array}{l}90 \\
90 \\
90\end{array}$ & $\begin{array}{l}36.3 \\
28.3 \\
13.3 \\
\end{array}$ & $\begin{array}{l}25.1 \\
20.0 \\
10.3\end{array}$ & $\begin{array}{l}0.13 \\
0.28 \\
0.56\end{array}$ & $\begin{array}{l}3.5 \\
3.5 \\
3.5\end{array}$ & $\begin{array}{l}0.39 \\
0.32 \\
0.20\end{array}$ & $\begin{array}{l}\mathrm{s} \\
\mathrm{s} \\
\mathrm{f}\end{array}$ \\
\hline $\begin{array}{l}\text { IV } \\
\mathrm{fa} 07 \\
\mathrm{fa} 08 \\
\mathrm{fa} 09\end{array}$ & $\begin{array}{l}0.45 \\
0.45 \\
0.45 \\
\end{array}$ & $\begin{array}{l}0.48 \\
0.51 \\
0.54 \\
\end{array}$ & $\begin{array}{l}0.71(0.45) \\
0.71(0.45) \\
0.71(0.45) \\
\end{array}$ & $\begin{array}{l}2.0 \\
2.0 \\
2.0 \\
\end{array}$ & $\begin{array}{l}30 \\
45 \\
60 \\
\end{array}$ & $\begin{array}{l}90 \\
90 \\
90 \\
\end{array}$ & $\begin{array}{c}24.8 \\
13.3 \\
5.3 \\
\end{array}$ & $\begin{array}{c}19.8 \\
10.2 \\
3.3 \\
\end{array}$ & $\begin{array}{l}0.37 \\
0.56 \\
0.74 \\
\end{array}$ & $\begin{array}{l}1.6 \\
1.6 \\
1.6 \\
\end{array}$ & $\begin{array}{l}0.63 \\
0.44 \\
0.26 \\
\end{array}$ & $\begin{array}{c}\mathrm{f} \\
\mathrm{fp} \\
\mathrm{t}\end{array}$ \\
\hline $\begin{array}{c}\mathrm{V} \\
\mathrm{fa} 10 \\
\mathrm{fa} 11 \\
\mathrm{fa} 12\end{array}$ & $\begin{array}{c}0.1 \\
0.3 \\
0.45\end{array}$ & $\begin{array}{l}0.10 \\
0.36 \\
0.76\end{array}$ & $\begin{array}{l}0.63(0.40) \\
1.88(1.20) \\
2.83(1.80)\end{array}$ & $\begin{array}{l}0.5 \\
0.5 \\
0.5\end{array}$ & $\begin{array}{c}0 \\
15 \\
30\end{array}$ & $\begin{array}{l}90 \\
90 \\
90\end{array}$ & $\begin{array}{l}17.4 \\
28.3 \\
24.8\end{array}$ & $\begin{array}{l}11.3 \\
20.0 \\
19.8\end{array}$ & $\begin{array}{c}0 \\
0.28 \\
0.37\end{array}$ & $\begin{array}{l}8.0 \\
0.9 \\
0.4\end{array}$ & $\begin{array}{l}0.20 \\
1.28 \\
2.50\end{array}$ & $\begin{array}{l}\mathrm{s} \\
\mathrm{s} \\
\mathrm{f}\end{array}$ \\
\hline $\begin{array}{c}\text { VI } \\
\text { fa13 } \\
\text { fa } 14 \\
\text { fa15 }\end{array}$ & $\begin{array}{c}0.1 \\
0.3 \\
0.45 \\
\end{array}$ & $\begin{array}{l}0.10 \\
0.32 \\
0.55 \\
\end{array}$ & $\begin{array}{l}0.31(0.20) \\
0.94(0.60) \\
1.41(0.90)\end{array}$ & $\begin{array}{l}1.0 \\
1.0 \\
1.0\end{array}$ & $\begin{array}{c}0 \\
15 \\
30 \\
\end{array}$ & $\begin{array}{l}90 \\
90 \\
90 \\
\end{array}$ & $\begin{array}{l}17.4 \\
28.3 \\
24.8 \\
\end{array}$ & $\begin{array}{l}11.3 \\
20.0 \\
19.8\end{array}$ & $\begin{array}{c}0 \\
0.28 \\
0.37\end{array}$ & $\begin{array}{c}15.9 \\
1.8 \\
0.8\end{array}$ & $\begin{array}{l}0.10 \\
0.64 \\
1.25\end{array}$ & $\begin{array}{l}\mathrm{s} \\
\mathrm{s} \\
\mathrm{f}\end{array}$ \\
\hline $\begin{array}{l}\text { VII } \\
\mathrm{fa} 19 \\
\mathrm{fa} 20 \\
\mathrm{fa} 21 \\
\mathrm{fa} 07 \\
\mathrm{fa} 34 \\
\mathrm{fa} 33 \\
\mathrm{fa} 22\end{array}$ & $\begin{array}{l}0.45 \\
0.45 \\
0.45 \\
0.45 \\
0.45 \\
0.45 \\
0.45 \\
\end{array}$ & $\begin{array}{l}0.33 \\
0.37 \\
0.42 \\
0.48 \\
0.48 \\
0.48 \\
0.52 \\
\end{array}$ & $\begin{array}{l}0.71(0.45) \\
0.71(0.45) \\
0.71(0.45) \\
0.71(0.45) \\
0.71(0.45) \\
0.71(0.45) \\
0.71(0.45) \\
\end{array}$ & $\begin{array}{l}2.0 \\
2.0 \\
2.0 \\
2.0 \\
2.0 \\
2.0 \\
2.0 \\
\end{array}$ & $\begin{array}{l}30 \\
30 \\
30 \\
30 \\
30 \\
30 \\
30 \\
\end{array}$ & $\begin{array}{c}30 \\
50 \\
70 \\
90 \\
100 \\
105 \\
110 \\
\end{array}$ & $\begin{array}{l}52.7 \\
42.4 \\
32.2 \\
24.8 \\
28.0 \\
30.0 \\
32.2 \\
\end{array}$ & $\begin{array}{l}32.9 \\
26.5 \\
21.8 \\
19.8 \\
20.4 \\
21.0 \\
21.8 \\
\end{array}$ & $\begin{array}{l}0.37 \\
0.37 \\
0.37 \\
0.37 \\
0.37 \\
0.37 \\
0.37 \\
\end{array}$ & $\begin{array}{l}1.6 \\
1.6 \\
1.6 \\
1.6 \\
1.6 \\
1.6 \\
1.6 \\
\end{array}$ & $\begin{array}{l}0.82 \\
0.72 \\
0.65 \\
0.63 \\
0.63 \\
0.64 \\
0.65 \\
\end{array}$ & $\begin{array}{l}\mathrm{s}^{*} \\
\mathrm{~s}^{*} \\
\mathrm{~s}^{*} \\
\mathrm{f} \\
\mathrm{s}^{*} \\
\mathrm{~s}^{*} \\
\mathrm{~s}^{*}\end{array}$ \\
\hline $\begin{array}{l}\text { VIII } \\
\text { fa23 } \\
\mathrm{fa} 24 \\
\mathrm{fa} 25 \\
\mathrm{fa} 15 \\
\mathrm{fa} 30 \\
\mathrm{fa} 32 \\
\mathrm{fa} 26\end{array}$ & $\begin{array}{l}0.45 \\
0.45 \\
0.45 \\
0.45 \\
0.45 \\
0.45 \\
0.45 \\
\end{array}$ & $\begin{array}{l}0.24 \\
0.35 \\
0.45 \\
0.55 \\
0.55 \\
0.55 \\
0.62 \\
\end{array}$ & $\begin{array}{l}1.41(0.90) \\
1.41(0.90) \\
1.41(0.90) \\
1.41(0.90) \\
1.41(0.90) \\
1.41(0.90) \\
1.41(0.90)\end{array}$ & $\begin{array}{l}1.0 \\
1.0 \\
1.0 \\
1.0 \\
1.0 \\
1.0 \\
1.0\end{array}$ & $\begin{array}{l}30 \\
30 \\
30 \\
30 \\
30 \\
30 \\
30 \\
\end{array}$ & $\begin{array}{c}30 \\
50 \\
70 \\
90 \\
100 \\
105 \\
110 \\
\end{array}$ & $\begin{array}{l}52.7 \\
42.4 \\
32.2 \\
24.8 \\
28.0 \\
30.0 \\
32.2 \\
\end{array}$ & $\begin{array}{l}32.9 \\
26.5 \\
21.8 \\
19.8 \\
20.4 \\
21.0 \\
21.8 \\
\end{array}$ & $\begin{array}{l}0.37 \\
0.37 \\
0.37 \\
0.37 \\
0.37 \\
0.37 \\
0.37 \\
\end{array}$ & $\begin{array}{l}0.8 \\
0.8 \\
0.8 \\
0.8 \\
0.8 \\
0.8 \\
0.8 \\
\end{array}$ & $\begin{array}{l}1.65 \\
1.44 \\
1.30 \\
1.25 \\
1.27 \\
1.28 \\
1.30 \\
\end{array}$ & $\begin{array}{c}\mathrm{s}^{*} \\
\mathrm{~s}^{*} \\
\mathrm{~s}^{*} \\
\mathrm{f} \\
\mathrm{s}^{*} \\
\mathrm{~s}^{*} \\
\mathrm{~s}^{*}\end{array}$ \\
\hline $\begin{array}{l}\text { IX } \\
\text { fa36 } \\
\text { fa37 } \\
\text { fa38 }\end{array}$ & $\begin{array}{l}0.32 \\
0.32 \\
0.32\end{array}$ & $\begin{array}{l}0.35 \\
0.31 \\
0.38\end{array}$ & 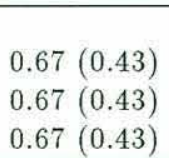 & $\begin{array}{l}1.5 \\
1.5 \\
1.5\end{array}$ & $\begin{array}{l}30 \\
30 \\
30\end{array}$ & $\begin{array}{c}90 \\
75 \\
105\end{array}$ & $\begin{array}{l}15.2 \\
20.4 \\
20.4\end{array}$ & $\begin{array}{l}11.7 \\
13.2 \\
13.2\end{array}$ & $\begin{array}{l}0.52 \\
0.52 \\
0.52\end{array}$ & $\begin{array}{l}2.4 \\
2.4 \\
2.4\end{array}$ & $\begin{array}{l}0.32 \\
0.34 \\
0.34\end{array}$ & $\begin{array}{l}f \\
f \\
f\end{array}$ \\
\hline
\end{tabular}

Table 4.1: Summary of foil experiment cases, $R_{c}=1100 . S t_{A}$ and $S t_{T E}$ are the Strouhal numbers based on heave double amplitude and trailing edge excursion respectively. $\alpha(t)$ profiles: 's' smooth sinusoid, 'f' smooth square wave, 'fp' double peaked square wave, 't' triple peaked sine wave, ' $\mathrm{s}$ ', distorted sine wave. 


\begin{tabular}{|c|c|c|c|c|c|c|c|c|}
\hline case & $\left|\frac{\overline{u-U}}{U}\right|_{\max }$ & $\frac{h}{c}$ & $\begin{array}{l}S t_{h} \\
\frac{h f}{U}\end{array}$ & $\begin{array}{l}\text { l.e. } \\
\text { sep }\end{array}$ & $\Gamma_{\text {wake }}^{*}$ & $\Gamma_{D S V}^{*}$ & $C_{f}$ & $\eta_{I}$ \\
\hline $\begin{array}{c}\mathrm{I} \\
\mathrm{fa} 04 \\
\mathrm{fa} 05 \\
\mathrm{fa} 06\end{array}$ & $\begin{array}{l}- \\
- \\
-\end{array}$ & $\begin{array}{l}- \\
- \\
-\end{array}$ & $\begin{array}{l}- \\
- \\
-\end{array}$ & $\begin{array}{c}4 \\
\text { none } \\
\text { none }\end{array}$ & $\begin{array}{l}- \\
- \\
-\end{array}$ & $\begin{array}{l}- \\
- \\
-\end{array}$ & $\begin{array}{l}- \\
- \\
-\end{array}$ & $\begin{array}{l}- \\
- \\
-\end{array}$ \\
\hline $\begin{array}{c}\text { II } \\
\text { fa16 } \\
\text { fa17 } \\
\text { fa18 }\end{array}$ & $\begin{array}{l}- \\
- \\
-\end{array}$ & $\begin{array}{l}- \\
- \\
-\end{array}$ & $\begin{array}{l}- \\
- \\
-\end{array}$ & $\begin{array}{c}2 \\
2 \\
\text { weak } 4\end{array}$ & $\begin{array}{l}- \\
- \\
-\end{array}$ & $\begin{array}{l}- \\
- \\
-\end{array}$ & $\begin{array}{l}- \\
- \\
-\end{array}$ & $\begin{array}{l}- \\
- \\
-\end{array}$ \\
\hline $\begin{array}{l}\text { III } \\
\mathrm{fa} 01 \\
\mathrm{fa} 02 \\
\mathrm{fa} 03\end{array}$ & $\begin{array}{c}0.37 \\
0.24 \\
-\end{array}$ & $\begin{array}{l}1.38 \\
1.84 \\
1.81\end{array}$ & $\begin{array}{l}0.21 \\
0.28 \\
0.27\end{array}$ & $\begin{array}{c}1,3 \\
1,3 \\
4\end{array}$ & $\begin{array}{l}2.0 \\
1.8 \\
0.9\end{array}$ & $\begin{array}{l}2.5 \\
1.4 \\
0.7\end{array}$ & $\begin{array}{l}1.04 \\
1.20 \\
0.28\end{array}$ & $\begin{array}{l}0.89 \\
0.88 \\
0.97\end{array}$ \\
\hline $\begin{array}{l}\text { IV } \\
\text { fa07 } \\
\text { fa08 } \\
\text { fa09 }\end{array}$ & $\begin{array}{c}0.36 \\
0.29 \\
-\end{array}$ & $\begin{array}{l}1.81 \\
2.05 \\
1.71\end{array}$ & $\begin{array}{l}0.41 \\
0.46 \\
0.38\end{array}$ & $\begin{array}{c}1 \\
1 \\
\text { none }\end{array}$ & $\begin{array}{c}2.0 \\
1.3 \\
-\end{array}$ & $\begin{array}{c}1.3 \\
1.6 \\
-\end{array}$ & $\begin{array}{l}2.98 \\
1.95 \\
-\end{array}$ & $\begin{array}{c}0.78 \\
0.83 \\
-\end{array}$ \\
\hline $\begin{array}{c}\mathrm{V} \\
\mathrm{fa} 10 \\
\mathrm{fa} 11 \\
\mathrm{fa} 12\end{array}$ & $\begin{array}{l}- \\
0.40 \\
1.12\end{array}$ & $\begin{array}{l}0.21 \\
0.70 \\
0.66\end{array}$ & $\begin{array}{l}0.04 \\
0.42 \\
0.59\end{array}$ & $\begin{array}{c}\text { weak } 4 \\
2 \\
\text { none }\end{array}$ & $\begin{array}{l}- \\
1.4 \\
2.4\end{array}$ & $\begin{array}{l}- \\
- \\
-\end{array}$ & $\begin{array}{c}- \\
0.77 \\
2.93\end{array}$ & $\begin{array}{c}- \\
0.71 \\
0.55\end{array}$ \\
\hline $\begin{array}{c}\text { VI } \\
\text { fa13 } \\
\text { fa14 } \\
\text { fa15 }\end{array}$ & $\begin{array}{c}- \\
0.39 \\
0.69\end{array}$ & $\begin{array}{l}- \\
0.60 \\
1.05\end{array}$ & $\begin{array}{c}- \\
0.18 \\
0.47\end{array}$ & $\begin{array}{c}2,3 \\
1 \\
1 \\
\end{array}$ & $\begin{array}{l}- \\
1.3 \\
1.6\end{array}$ & $\begin{array}{c}- \\
0.9 \\
1.5 \\
\end{array}$ & $\begin{array}{l}- \\
1.16 \\
1.86 \\
\end{array}$ & $\begin{array}{l}0.81 \\
0.74\end{array}$ \\
\hline $\begin{array}{l}\text { VII } \\
\text { fa } 19 \\
f a 20 \\
f a 21 \\
f a 07 \\
f a 34 \\
f a 33 \\
f a 22\end{array}$ & $\begin{array}{l}0.18 \\
0.38 \\
0.35 \\
0.36 \\
0.32 \\
0.34 \\
0.36\end{array}$ & $\begin{array}{l}0.26 \\
0.91 \\
1.25 \\
1.81 \\
2.07 \\
2.36 \\
2.28\end{array}$ & $\begin{array}{l}0.06 \\
0.20 \\
0.28 \\
0.41 \\
0.47 \\
0.53 \\
0.52\end{array}$ & $\begin{array}{c}1 \\
1,3 \\
1,3 \\
1 \\
1 \\
1 \\
1,3\end{array}$ & $\begin{array}{l}2.4 \\
2.2 \\
2.0 \\
2.0 \\
1.5 \\
1.5 \\
1.9\end{array}$ & $\begin{array}{c}2.8 \\
2.8 \\
1.9 \\
1.3 \\
1.2 \\
1.4 \\
-\end{array}$ & $\begin{array}{l}-0.04 \\
0.85 \\
1.78 \\
2.98 \\
2.09 \\
2.68 \\
2.59\end{array}$ & $\begin{array}{c}- \\
0.91 \\
0.84 \\
0.78 \\
0.82 \\
0.79 \\
0.80\end{array}$ \\
\hline $\begin{array}{l}\text { VIII } \\
\text { fa } 23 \\
\text { fa } 24 \\
\text { fa } 25 \\
\text { fa } 15 \\
\text { fa30 } \\
\text { fa32 } \\
\text { fa26 }\end{array}$ & $\begin{array}{c}- \\
0.53 \\
0.36 \\
0.69 \\
0.73 \\
0.68 \\
0.81\end{array}$ & $\begin{array}{c}- \\
0.53 \\
0.84 \\
1.05 \\
1.18 \\
1.25 \\
1.15\end{array}$ & $\begin{array}{c}- \\
0.24 \\
0.38 \\
0.47 \\
0.53 \\
0.56 \\
0.52\end{array}$ & $\begin{array}{c}1 \\
1 \\
1 \\
1 \\
1 \\
1 \\
\text { weak } 2\end{array}$ & $\begin{array}{l}- \\
1.9 \\
1.5 \\
1.6 \\
2.0 \\
2.2 \\
2.3\end{array}$ & $\begin{array}{c}- \\
1.2 \\
1.2 \\
1.5 \\
- \\
- \\
-\end{array}$ & $\begin{array}{c}- \\
0.97 \\
0.94 \\
1.86 \\
3.37 \\
2.72 \\
3.55\end{array}$ & $\begin{array}{c}- \\
0.83 \\
0.84 \\
0.74 \\
0.65 \\
0.68 \\
0.64\end{array}$ \\
\hline $\begin{array}{c}\text { IX } \\
\text { fa36 } \\
\text { fa37 } \\
\text { fa38 }\end{array}$ & $\begin{array}{l}0.15 \\
0.13 \\
0.17\end{array}$ & $\begin{array}{l}1.57 \\
1.67 \\
1.50\end{array}$ & $\begin{array}{l}0.33 \\
0.35 \\
0.32\end{array}$ & $\begin{array}{l}1 \\
1 \\
1\end{array}$ & $\begin{array}{l}1.1 \\
0.9 \\
1.0\end{array}$ & $\begin{array}{l}1.3 \\
1.1 \\
1.1\end{array}$ & $\begin{array}{l}0.70 \\
0.34 \\
0.77\end{array}$ & $\begin{array}{l}0.90 \\
0.95 \\
0.90\end{array}$ \\
\hline
\end{tabular}

Table 4.2: Summary of foil experiment results, $R_{c}=1100$. Leading edge separation type: (1) well separated vortex rolls along body, (2) less organized separated vortex rolls along body, (3) secondary leading edge vortices present, (4) separated region, does not roll up until trailing edge. $h$ is the wake width and $S t_{h}$ is the Strouhal number based on wake width. Case kinematics are given in Table 4.1. 
differences between vortices used in the average circulation calculation. The typical standard deviation of these measurements from the mean is under three percent of the mean value for well behaved wakes. If the wake is highly mixed, the variability of the circulation increases to 10 to 15 percent. In general, the circulation is a stable, accurate measure of the wake strength.

The thrust coefficient $C_{f}$ is computed by integrating across the average wake velocity profile using equation (4.6) to get the thrust, and then normalizing with equation (4.10). The calculation is performed numerically using a trapezoidal approximation between $y=$ $\pm 1.5 c$. The size of the experimental view sets this limit on the integration bounds. Although some of the experiments have flow features which approach these bounds, the average wake velocity goes to zero as required at the boundary of the integration. The robustness of the thrust coefficient calculation was verified by comparing the results to those obtained with duplicate experiments. The maximum variability observed was $\pm 5 \%$, which indicates that the wake momentum flux is a repeatable measure of the thrust force.

The ideal efficiency given in the last column of Table 4.2 is computed using the actuator disk formulation given in section 1.1.1. The actuator disk thrust coefficient $C_{T}$ in terms of $C_{f}$ is

$$
C_{T}=\frac{C_{f}}{\frac{2 A}{c}}
$$

The ideal efficiency $\eta_{I}$ is computed using Equation (1.2).

\subsubsection{Groups I through IV}

Figures 4-10 through 4-12 show the velocity fields in the wake of the foil for similar motions at three different Strouhal numbers. Large heave amplitude $\left(\frac{2 A}{c}=2.0\right)$ and moderate angle of attack $\left(10.4^{\circ}<\alpha_{\max }<13.3^{\circ}\right)$ are expected to produce a thrust wake.

The velocity data very near the foil and in the shadow are removed as discussed in Chapter 2 to prevent spurious results. A reference vector in the lower left hand corner of each plot gives a measure of the tow speed in relation to the displayed vectors. Unless otherwise noted, all velocity vector fields in this chapter are plotted using the same absolute scale. Low velocities appear as short arrows, high velocities with longer arrows. In the following figures, the foil moves from left to right and is shown at the minimum position of its heave trajectory. 


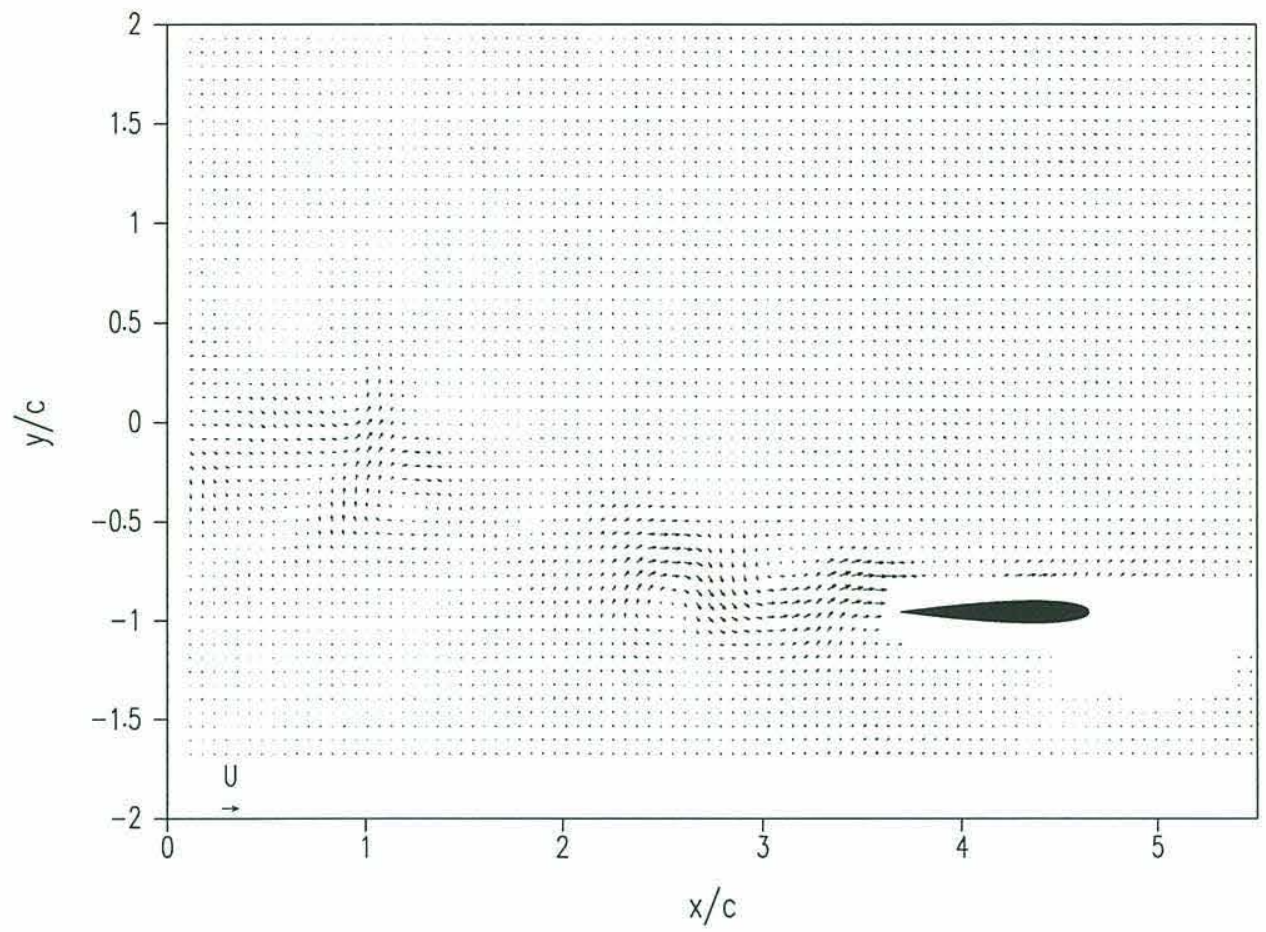

Figure 4-10: DPIV velocity data for case fa05. Foil at minimum heave position. $S t_{T E}=0.10, \frac{2 A}{c}=2.0, \theta_{\circ}=7^{\circ}, \alpha_{\max }=10.4^{\circ}, \phi=90^{\circ}$.

Figure 4-10 shows the low Strouhal number case, $S t_{T E}=0.10$. The weak Kármán street is confined to the path of the foil. Although the MIT experimental data (Figure 4-1) predict minuscule thrust for this Strouhal number, it is not evident from the wake momentum that any thrust is generated. As the Strouhal number is increased (Figure 4-11) to 0.30, the suction side of the foil separates forming a laminar bubble. The bubble sluggishly organizes into a weak vortex which interacts with trailing edge generated vorticity to form a weakly mixed wake. In Figure 4-11 a leading edge vortex persists at $\frac{x}{c}=1.0, \frac{y}{c}=0.6$.

As the Strouhal number is further increased to $S t_{T E}=0.50$ (Figure 4-12), an organized leading edge vortex forms which rolls along the foil surface. Small secondary leading edge separation is also seen as the foil begins its upward and downward trajectories after the point of zero pitch. Shed leading edge vortices persist in the wake for several wavelengths. Considerable streamwise momentum indicates strong thrust.

Figures 4-13 through 4-15 show the effect of increased angle of attack for roughly the same Strouhal numbers and heave amplitude. Because the pitch amplitude is required to be a discrete value for the oscillation apparatus, the angles of attack are not equal for these runs. In order to specify the Strouhal number, we suffer a limited selection of possible angles of attack. Hence, it is more appropriate to compare these results with the previous 


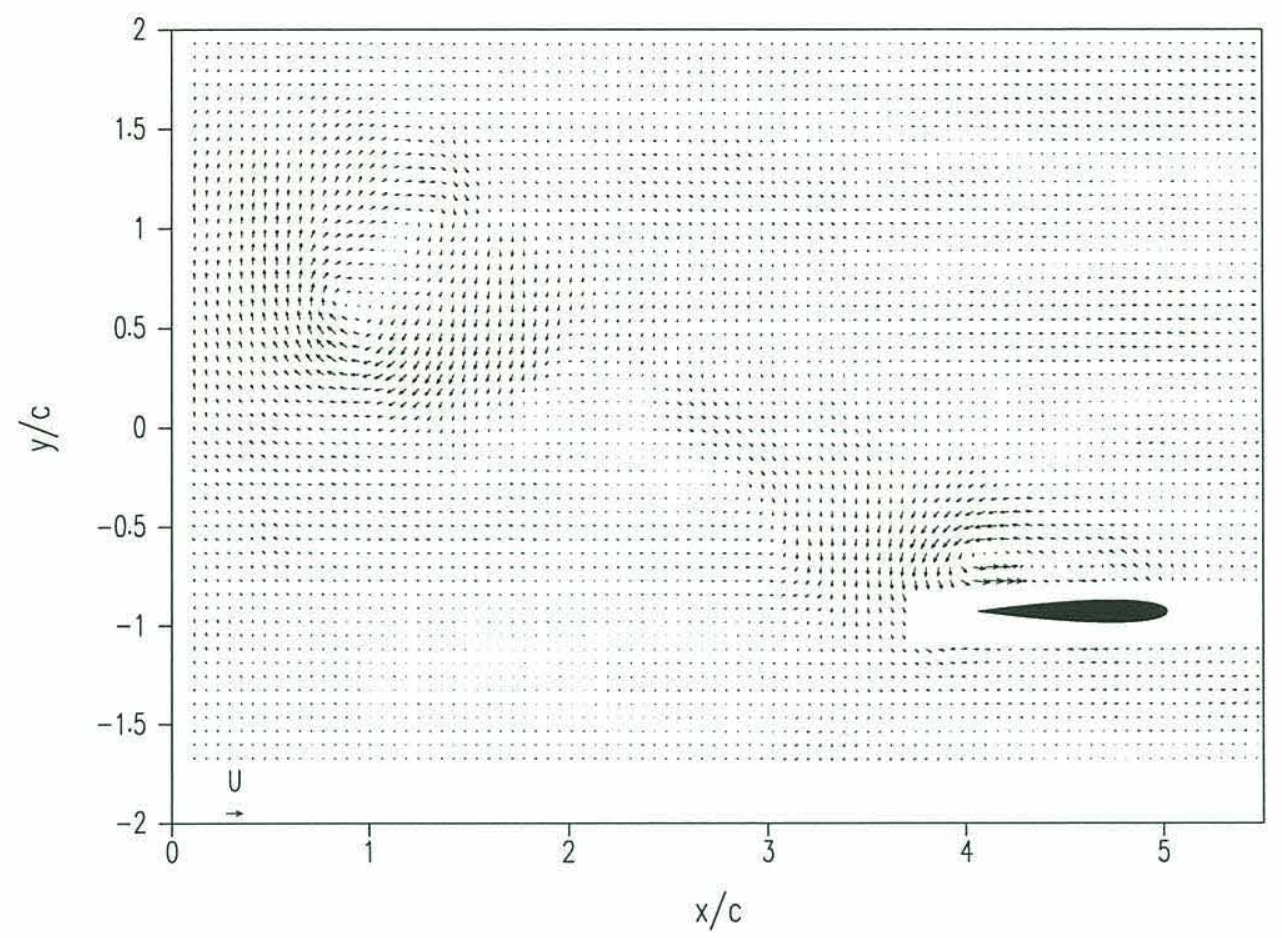

Figure 4-11: DPIV velocity data for case fa03. Foil at minimum heave position. $S t_{T E}=0.30, \frac{2 A}{c}=2.0, \theta_{\circ}=30^{\circ}, \alpha_{\max }=13.3^{\circ}, \phi=90^{\circ}$.

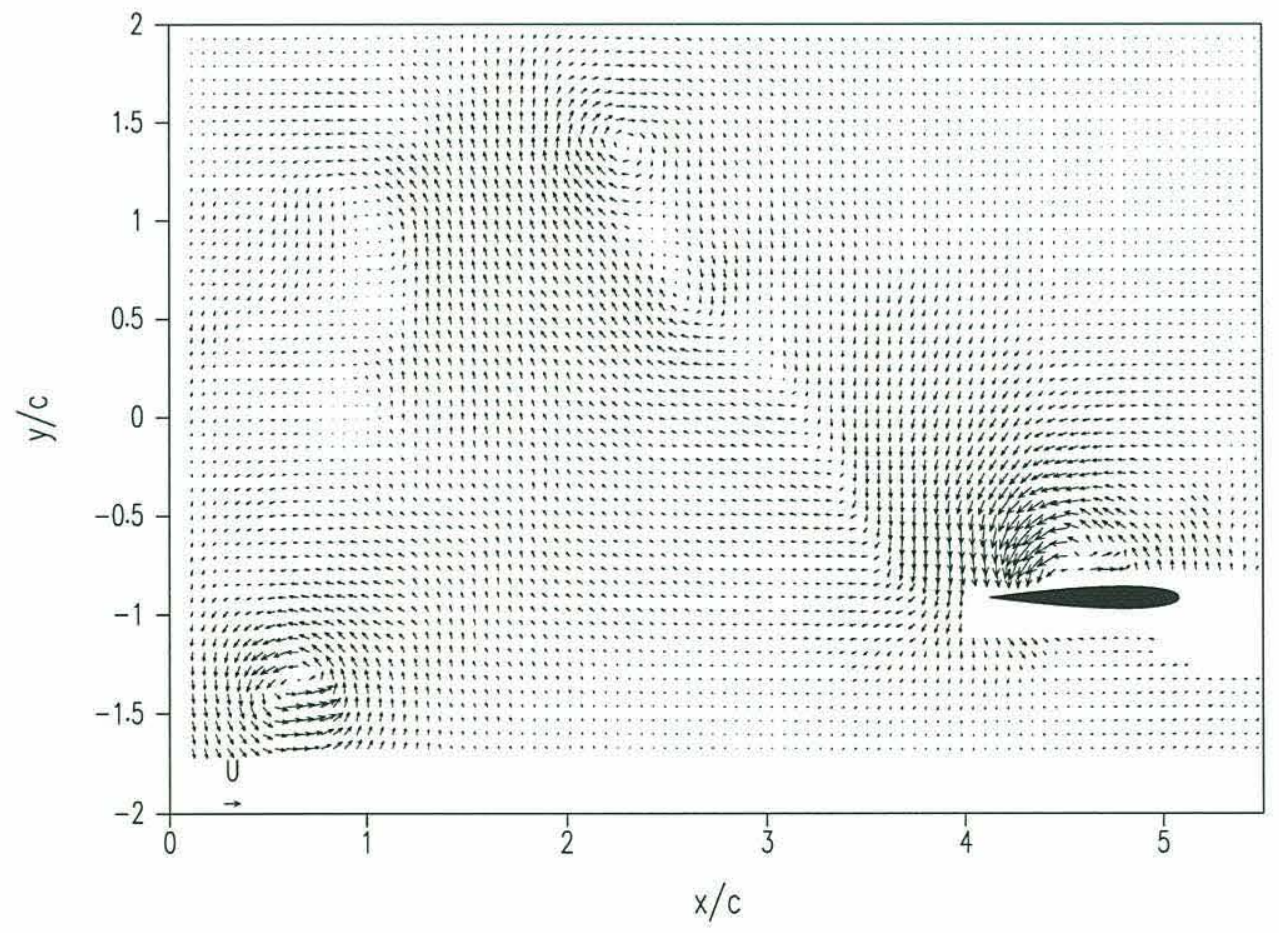

Figure 4-12: DPIV velocity data for case fa08. Foil at minimum heave position. $S t_{T E}=0.50, \frac{2 A}{c}=2.0, \theta_{\circ}=45^{\circ}, \alpha_{\max }=13.3^{\circ}, \phi=90^{\circ}$. 


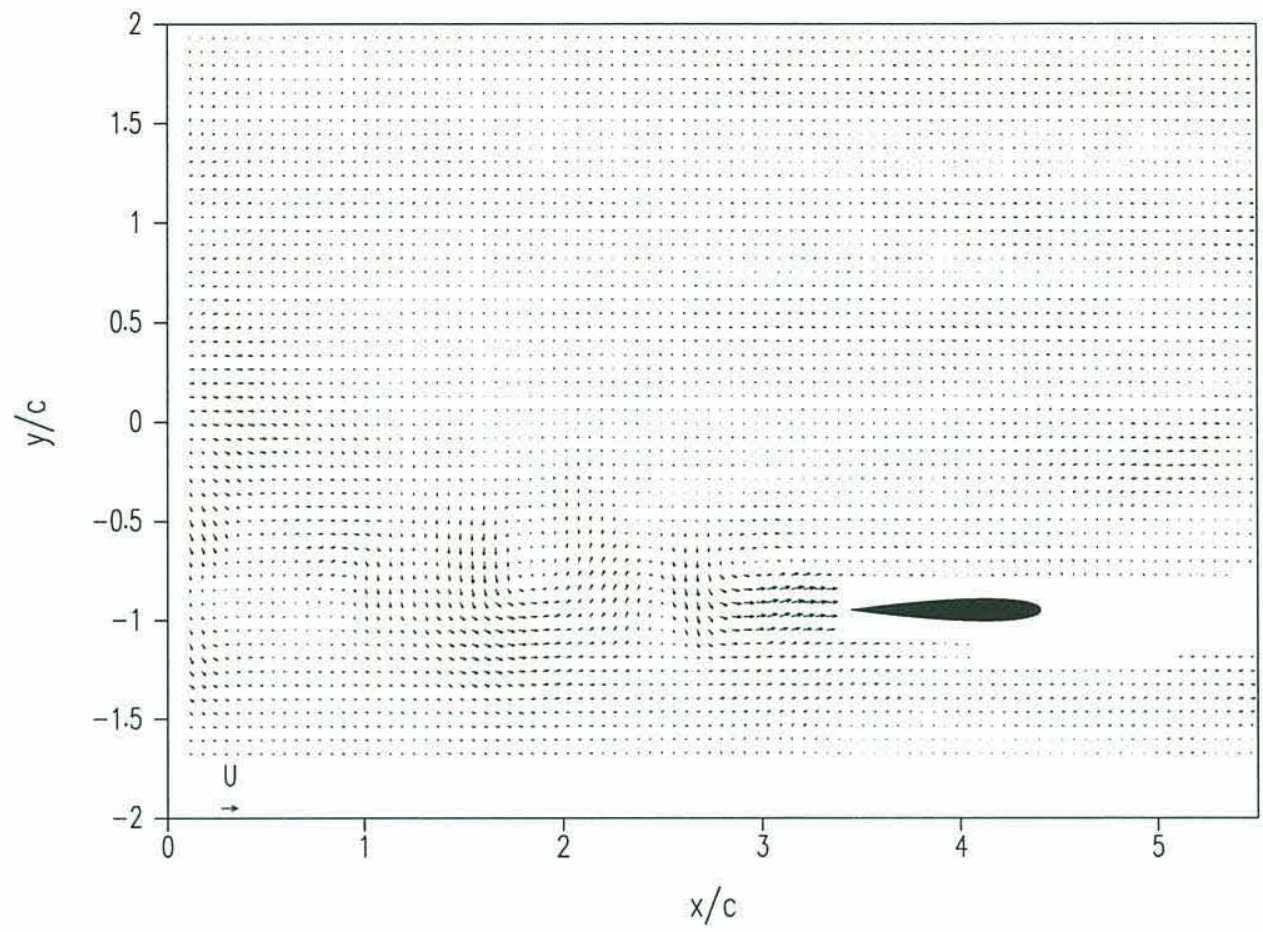

Figure 4-13: DPIV velocity data for case fa04. Foil at minimum heave position. $S t_{T E}=0.10, \frac{2 A}{c}=2.0, \theta_{\circ}=0^{\circ}, \alpha_{\max }=17.4^{\circ}, \phi=90^{\circ}$.

results at lower angles of attack.

Figure 4-13 shows the low Strouhal number $\left(S t_{T E}=0.10\right)$ velocity field for $\alpha_{\max }=$ $17.4^{\circ}$. Comparison with Figure 4-10 shows a nearly identical result, a wavy Kármán street along the foil path and a weakly mixed wake. The streamwise momentum indicates a drag wake. This case differs from the lower angle of attack case in that a leading edge bubble forms when the angle of attack is maximum at the heave zero crossing. This bubble breaks away from the foil in bluff-body type shedding, without forming a persistent vortex in the wake.

Figure 4-14 shows the velocity field for $S t_{T E}=0.30$ and $\alpha_{\max }=28.3^{\circ}$. A dynamic stall vortex forms near the point of maximum angle of attack at the heave zero crossing. The well formed DSV rolls along the foil and sheds from the trailing edge just as the foil is poised to change direction at the point of zero pitch. A small amount of secondary vorticity forms at this time at the leading edge. The DSV downwash suppresses trailing edge vortex shedding. The resulting wake is a wide $(2 A)$ thrust jet, dominated by shed leading edge vortices. Comparison with Figure 4-11 shows that increasing the angle of attack amplifies the DSV and the resulting wake intensity. At $\alpha_{\max }=13.3^{\circ}$ the DSV is sluggish to organize, whereas at $\alpha_{\max }=28.3^{\circ}$, the DSV rolls along and off of the foil in an organized, highly 


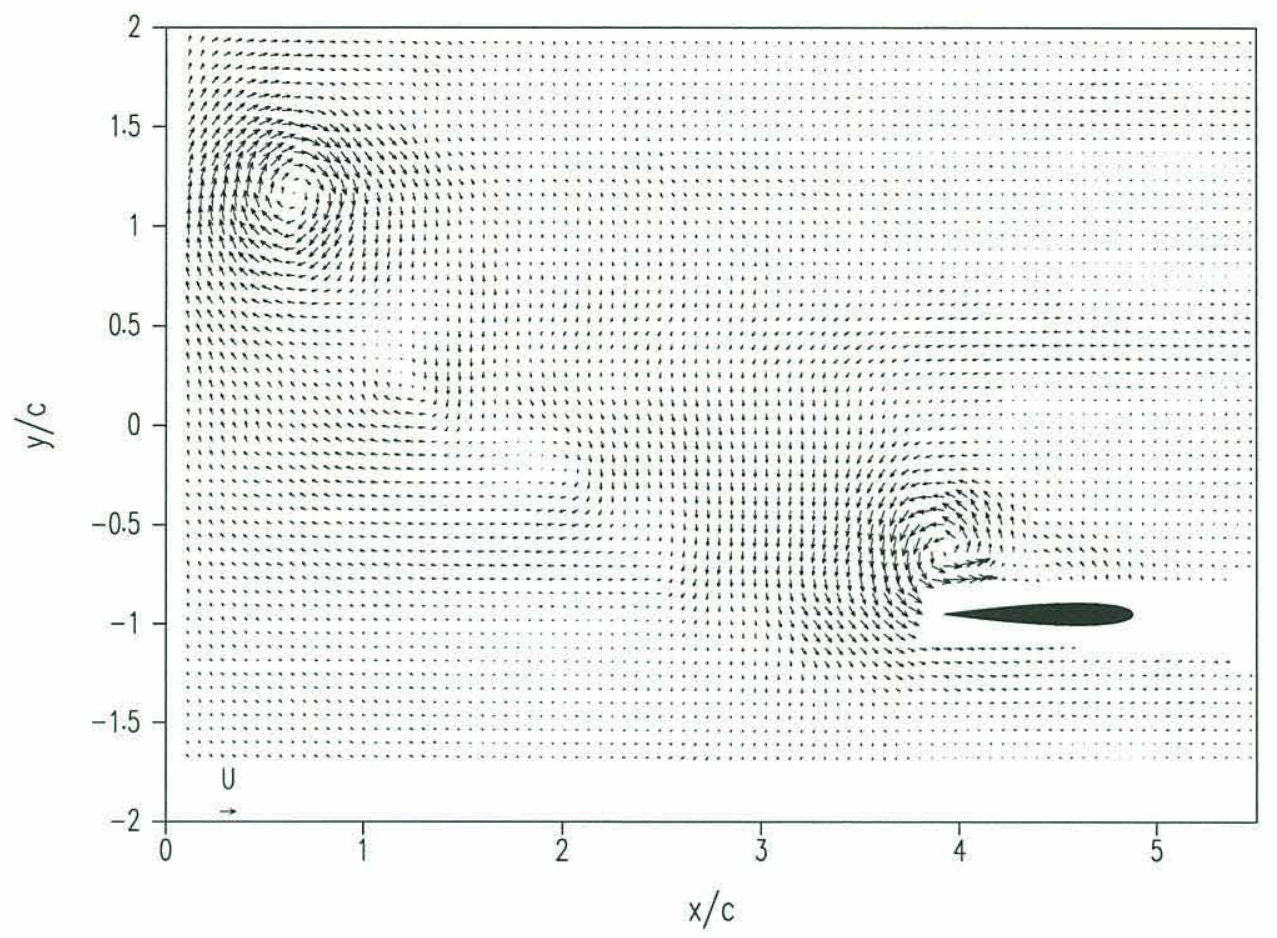

Figure 4-14: DPIV velocity data for case fa02. Foil at minimum heave position. $S t_{T E}=0.30, \frac{2 A}{c}=2.0, \theta_{\circ}=15^{\circ}, \alpha_{\max }=28.3^{\circ}, \phi=90^{\circ}$.

energized manner. The circulation values for both the wake and the stall vortices quantify this effect. Both $\Gamma_{w a k e}^{*}$ and $\Gamma_{D S V}^{*}$ double for the increase from $\alpha_{\max }=13.3^{\circ}$ to $28.3^{\circ}$. The centerline of the wake stagnates since the dominant vortices stay close to the position of maximum heave.

Figure 4-15 shows the velocity field for high Strouhal number, $S t_{T E}=0.50$, at $\alpha_{\max }=$ $24.8^{\circ}$. The wide jet wake is highly organized with considerable streamwise momentum. Comparison with Figure 4-12 confirms again that increasing the angle of attack amplifies the wake energy. The leading edge vortex is more defined although it has approximately the same strength as the lower angle of attack case. Again, the DSV's dominate the wake pattern and little vorticity is generated by the trailing edge. The shear layer left along the path of the trailing edge is marked by the change in sign of the vertical velocity component.

Comparison of cases fa07 and fa08 shows the effect of angle of attack shape on wake structure. In fa07, the angle of attack profile is a flattened sinusoid whereas in case fa08 it is flattened with additional corner peaks. The velocity fields reflect this difference very clearly (Figures 4-15 and 4-12). The flattened angle of attack profile produces a clean leading edge vortex and stable, organized wake. Even shear layer vorticity along the trailing edge path remains stable for at least half a wavelength. In contrast, the multi-peaked angle of attack 


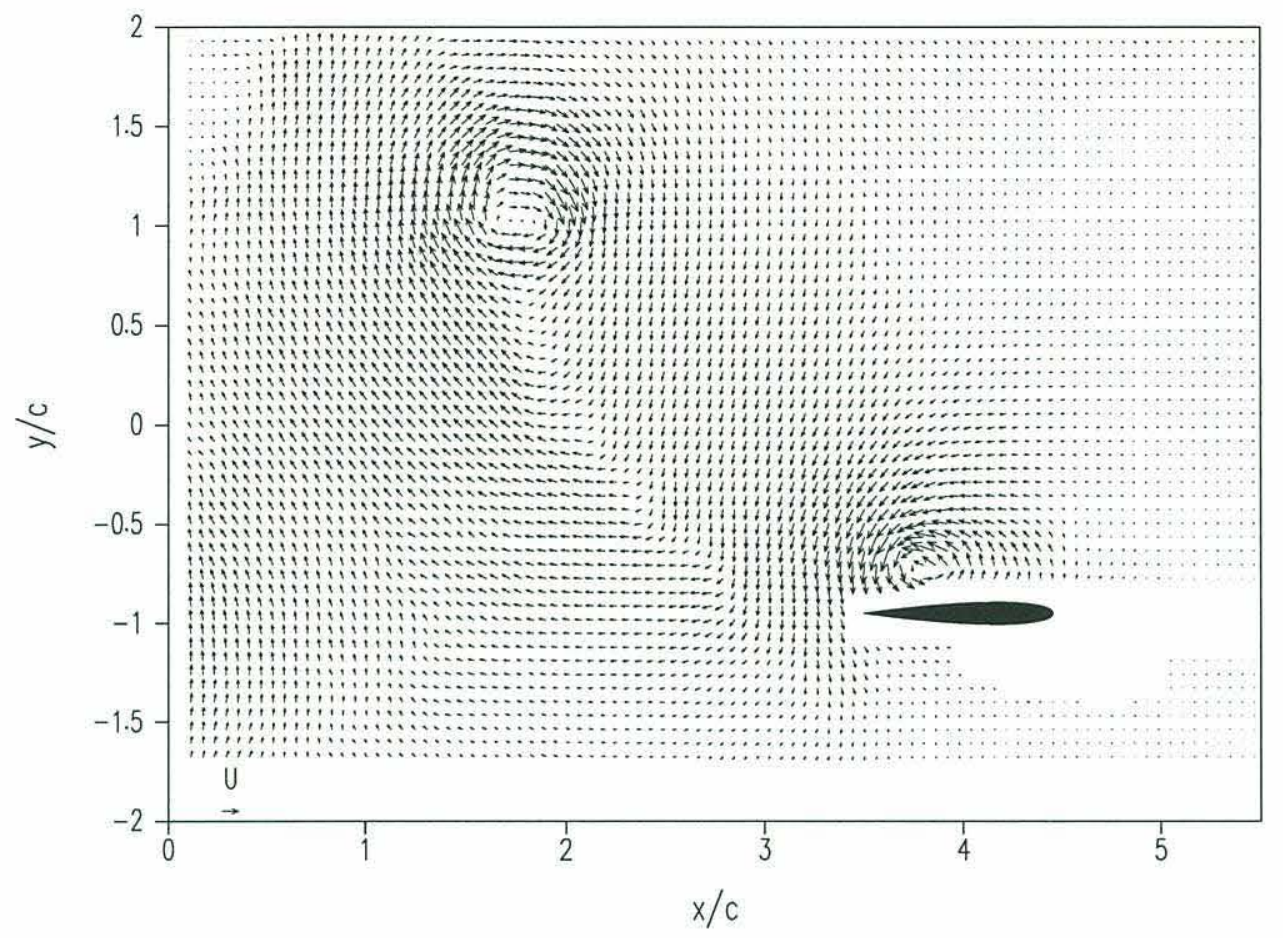

Figure 4-15: DPIV velocity data for case fa07. Foil at minimum heave position. $S t_{T E}=0.48, \frac{2 A}{c}=2.0, \theta_{\circ}=30^{\circ}, \alpha_{\max }=24.8^{\circ}, \phi=90^{\circ}$.

profile generates extra vorticity in the trailing edge path and a slightly mixed wake.

\subsubsection{Groups V and VI}

Figures 4-16 through 4-18 demonstrate the effect of low heave amplitude $\left(\frac{2 A}{c}=0.5\right)$ on the wake structure for fixed moderate angles of attack. In these figures the foil is shown in its maximum heave position. At low Strouhal number (Figure 4-16), the wake changes little compared to the high amplitude case. A laminar separation bubble forms which is drawn into the wake without without forming a distinct vortex. The wake is wavy and mixed with no streamwise momentum to indicate thrust.

As the Strouhal number is increased to 0.36 (Figure 4-17), the trailing edge dominates the wake structure by shedding two vortices per cycle in a reverse Kármán street jet pattern. Small leading edge vortices form which are absorbed into the trailing edge wake. The staggered array of vortices is persistent for several wavelengths. Although this motion has the same angle of attack and Strouhal number as case fa02 (Figure 4-14), leading edge effects are much less important.

Further increase of the Strouhal number generates the extremely strong jet wake shown 


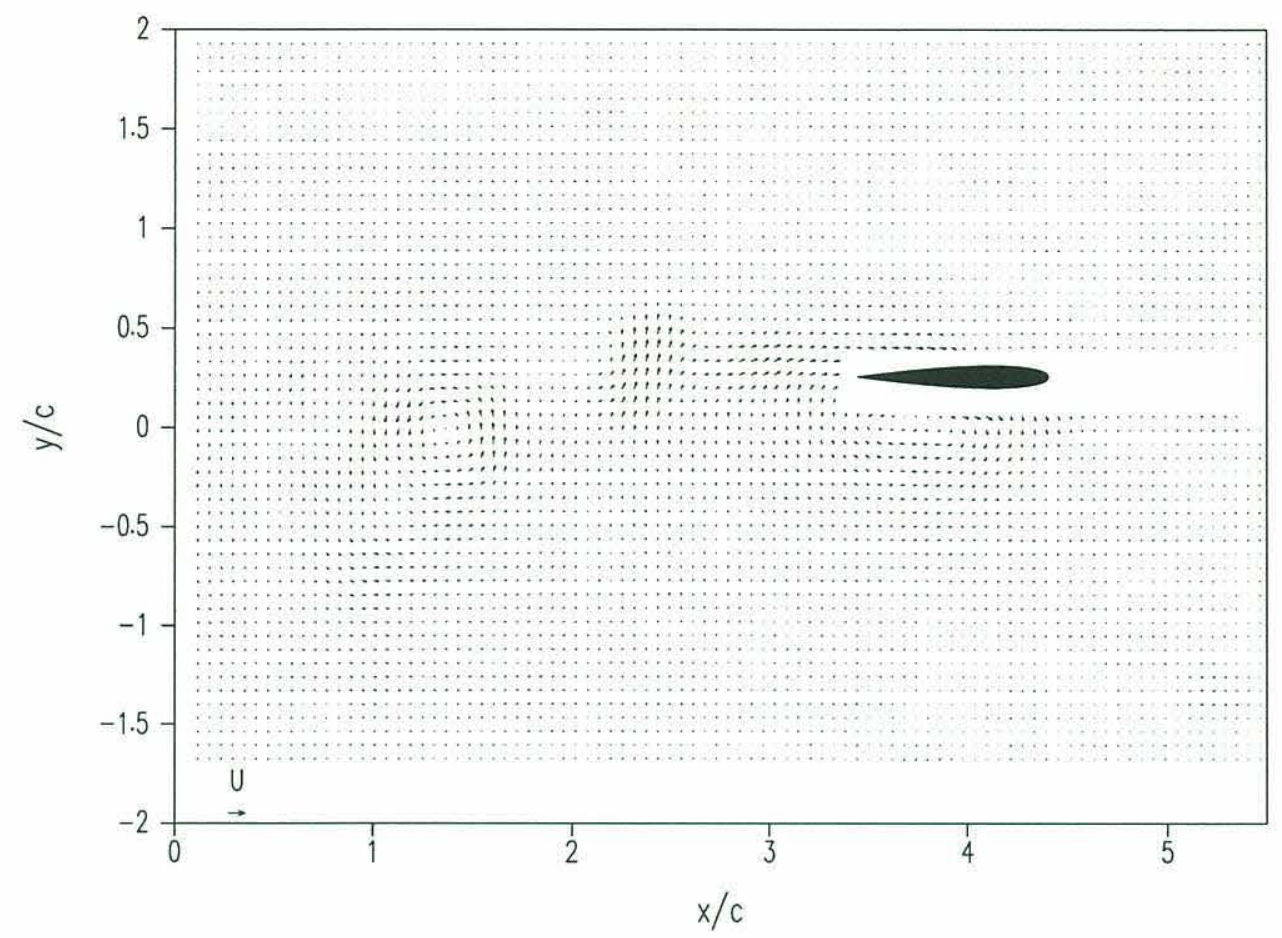

Figure 4-16: DPIV velocity data for case fa10. Foil at maximum heave position. $S t_{T E}=0.10, \frac{2 A}{c}=0.5, \theta_{\circ}=0^{\circ}, \alpha_{\max }=17.4^{\circ}, \phi=90^{\circ}$.

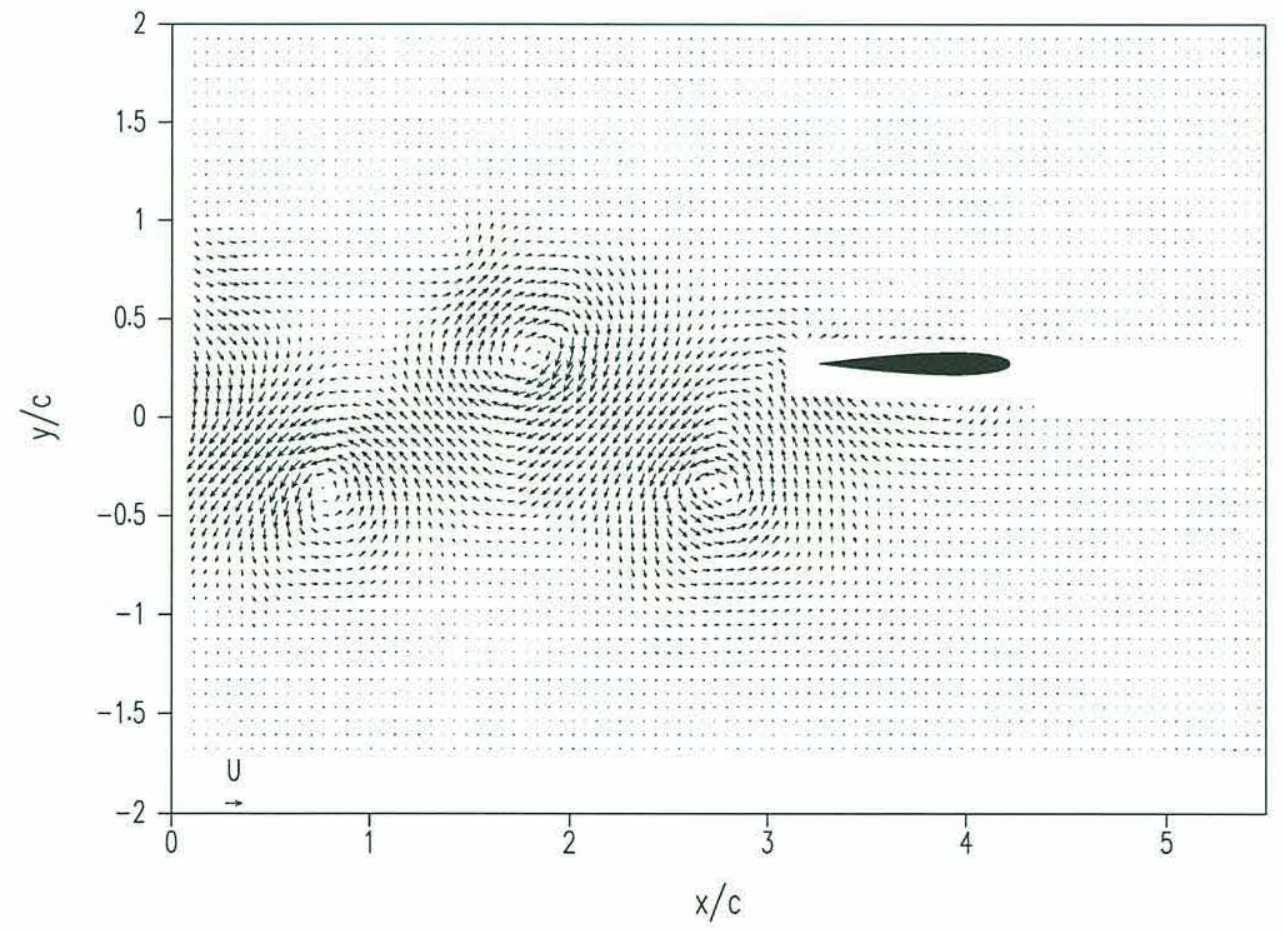

Figure 4-17: DPIV velocity data for case fa11. Foil at maximum heave position. $S t_{T E}=0.36, \frac{2 A}{c}=0.50, \theta_{\circ}=15^{\circ}, \alpha_{\max }=28.3^{\circ}, \phi=90^{\circ}$. 


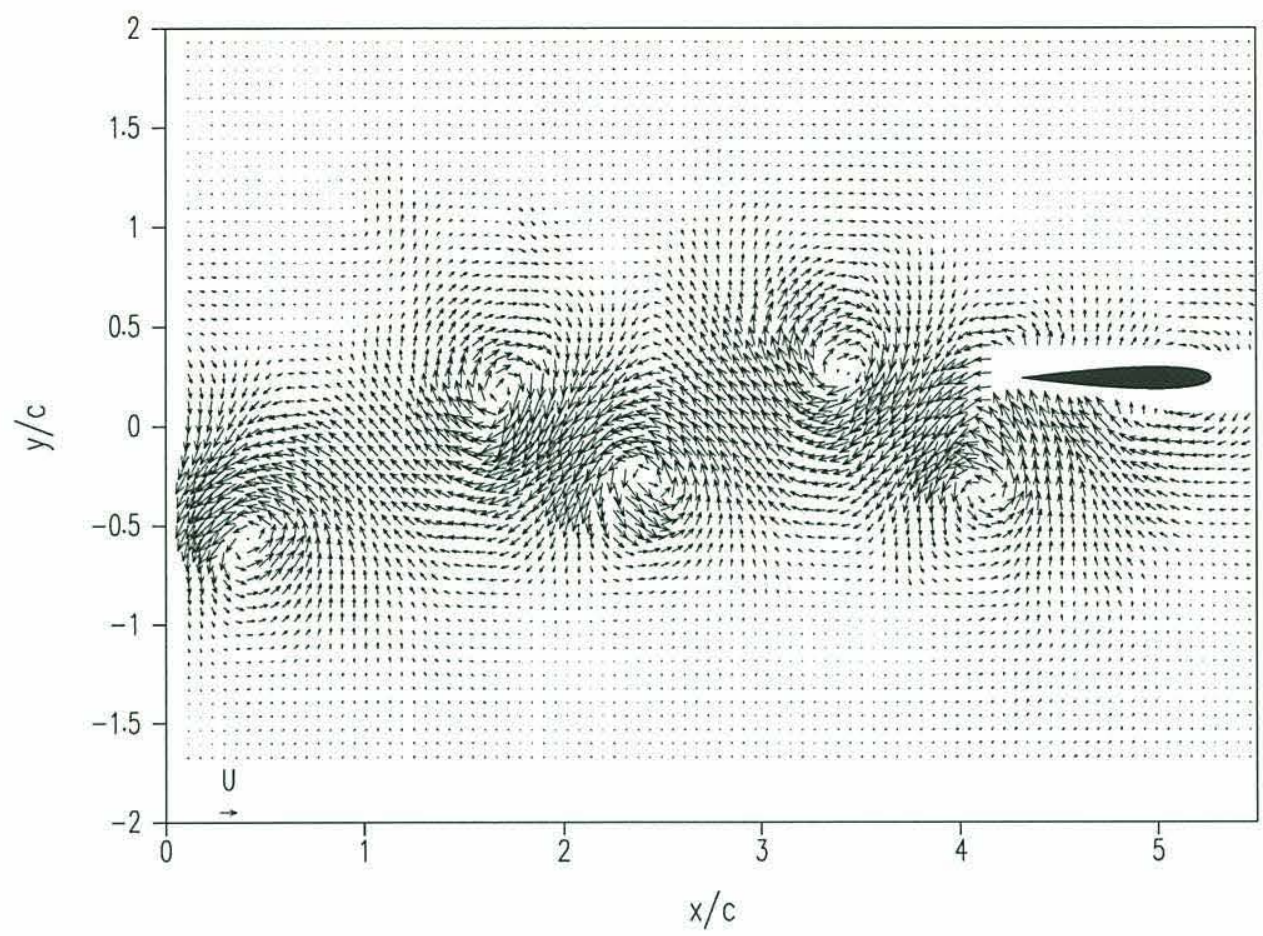

Figure 4-18: DPIV velocity data for case fa12. Foil at maximum heave position. $S t_{T E}=0.76, \frac{2 A}{c}=0.5, \theta_{\circ}=30^{\circ}, \alpha_{\max }=24.8^{\circ}, \phi=90^{\circ}$.

in Figure 4-18. This case illustrates the difficulty in defining an appropriate Strouhal number. The Strouhal number is $0.45,0.76$ and 0.59 based on heave double amplitude, trailing edge excursion and wake width respectively. The wake vortex strength is nearly double that for case fa11 at roughly half the Strouhal number and approximately the same angle of attack. No leading edge separation is observed for this case although the angle of attack is 24.8 . This result is in contrast to case fa07 (Figure 4-15) which has strong DSV formation and little trailing edge contribution for the same angle of attack and larger heave amplitude. Clearly, the curvature of the trajectory and the rate of change of the angle of attack play an important role in both leading and trailing edge effects. The jet wake for this case is very unstable spatially. The vortices are so highly energized that they tend to pair off and move away from the centerline. Slight adjustment $\left(<1^{\circ}\right)$ of the pitch amplitude setting caused the pairs to move either up or down from the centerline.

So far we have looked at low heave amplitude and high heave amplitude cases. Moderate heave amplitude $\left(\frac{2 A}{c}=1.0\right)$ results are shown in Figures 4-19 through 4-21 for similar Strouhal numbers and angles of attack as in the previous cases. Figure 4-19 shows case fa13 where the Strouhal number is 0.10 . Although one strong vortex appears in the wake, it is a Kármán type drag vortex which weakly mixes the wake. A sluggish separated region forms 


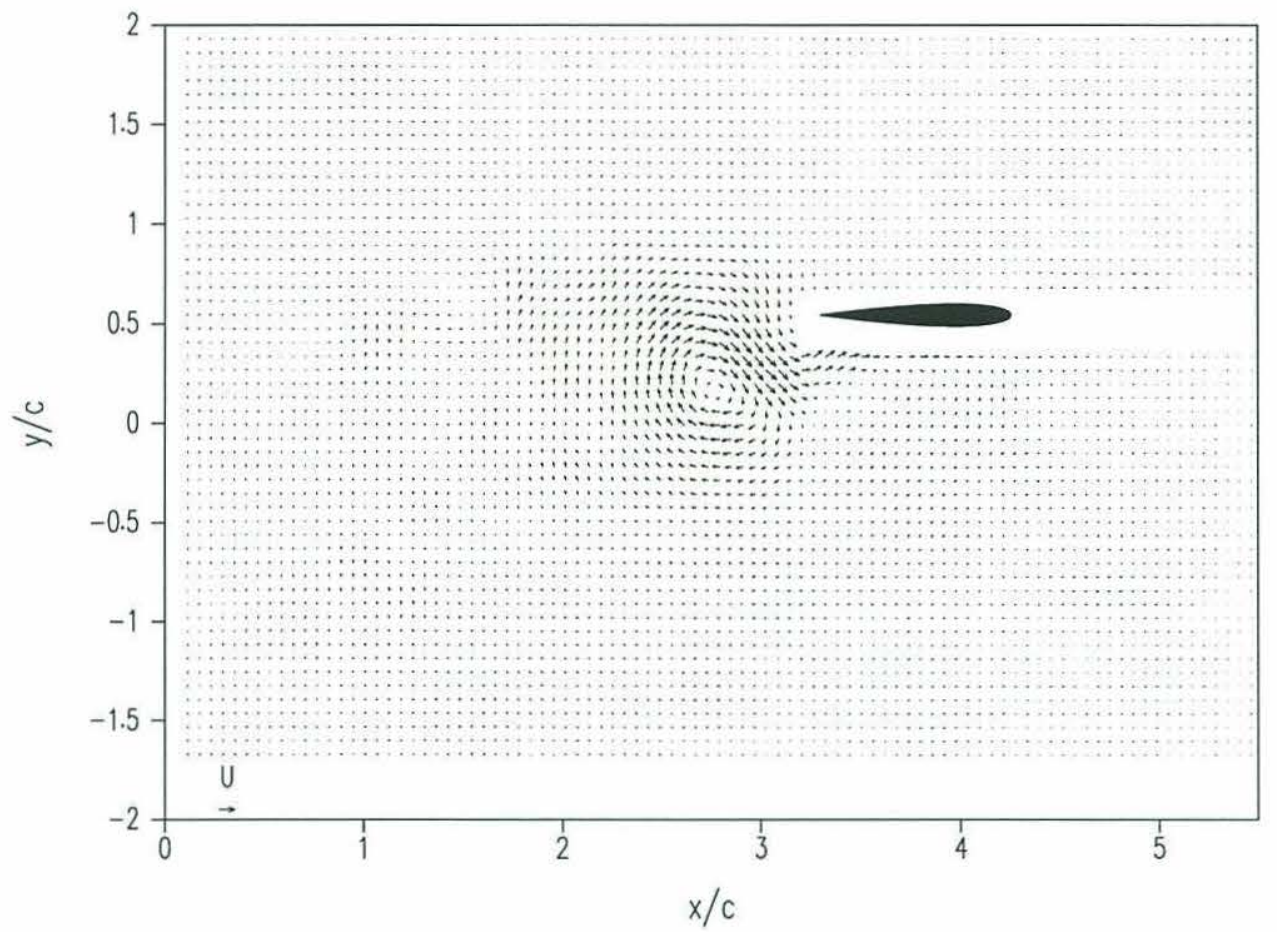

Figure 4-19: DPIV velocity data for case fa13. Foil at maximum heave position. $S t_{T E}=0.10, \frac{2 A}{c}=1.0, \theta_{\circ}=0^{\circ}, \alpha_{\max }=17.4^{\circ}, \phi=90^{\circ}$.

on the suction side of the foil which eventually moves into the wake.

At Strouhal number 0.32 (Figure 4-20), a single leading edge vortex rolls along the foil and leaves the trailing edge smoothly at the peaks in the heave motion. The foil appears to slalom around the separated leading edge vortices without generating any trailing edge vorticity.

As the Strouhal number is further increased to 0.55 (Figure 4-21), the jet pattern is amplified. Most of the vorticity is generated by the trailing edge which then combines with same signed leading edge vorticity. Again, an increase in the Strouhal number causes an increase in the strength of both the wake and stall vortices.

\subsubsection{Groups VII and VIII}

In groups VII and VIII we investigate the effect of changing the phase angle between heave and pitch. In all of the previous cases, the phase angle was $90^{\circ}$ with pitch leading heave in time. Linear theory and most authors agree that this phase angle should yield the most thrust. However, the recent MIT experimental results indicate that there may be an unknown mechanism which enhances efficiency and depends on the phasing of the motion. 


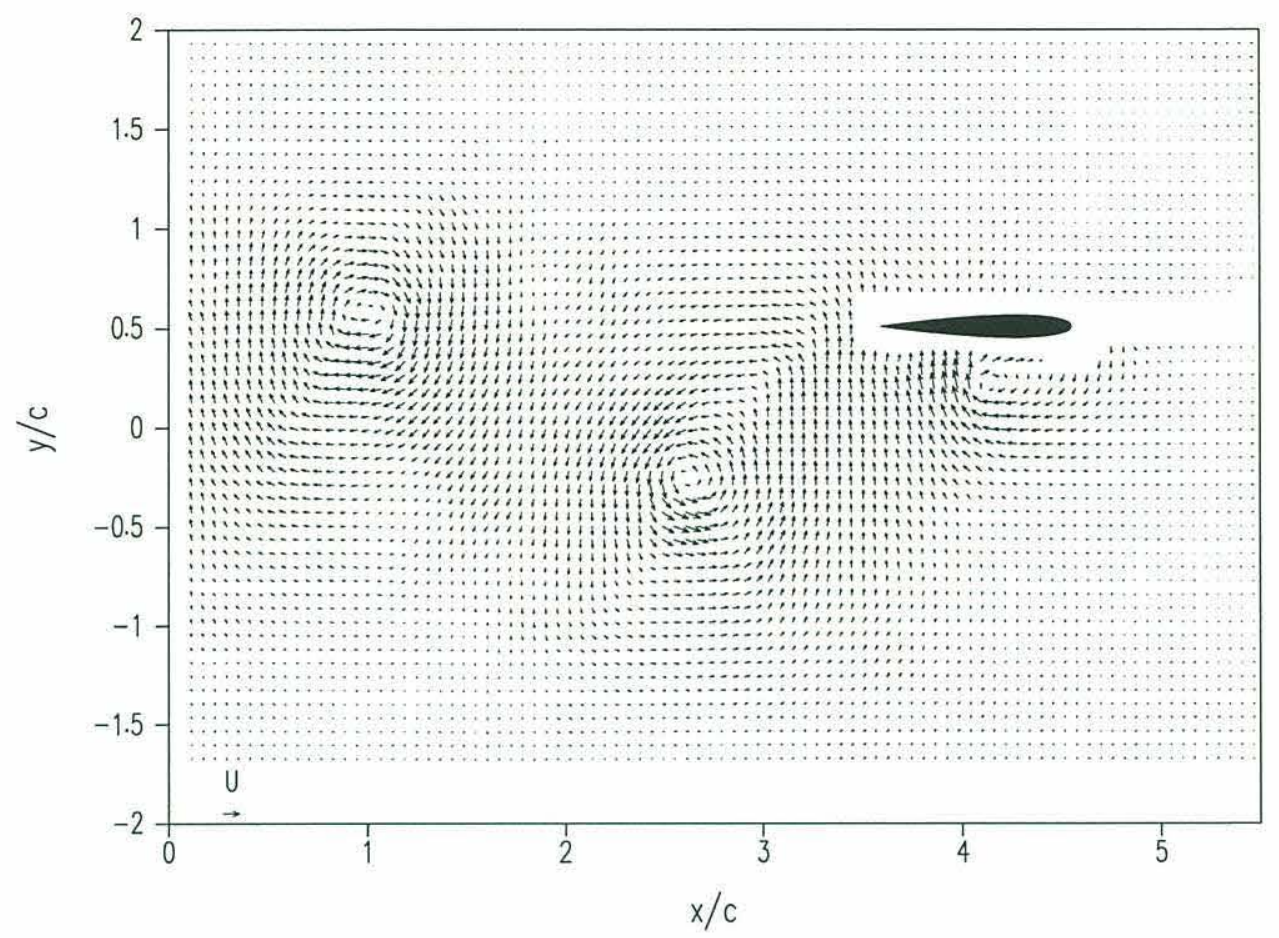

Figure 4-20: DPIV velocity data for case fa14. Foil at maximum heave position. $S t_{T E}=0.32, \frac{2 A}{c}=1.0, \theta_{\circ}=15^{\circ}, \alpha_{\max }=28.3^{\circ}, \phi=90^{\circ}$.

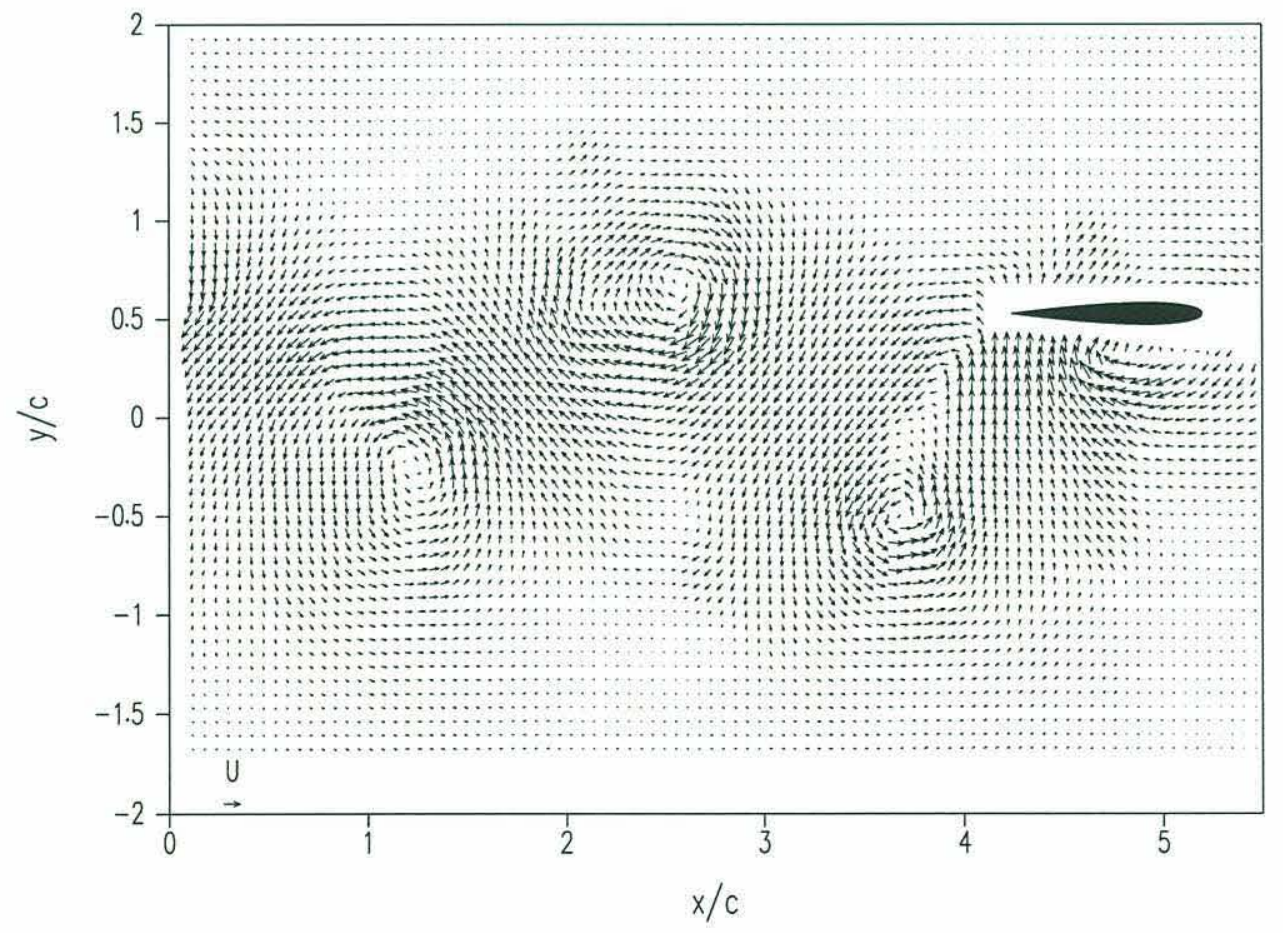

Figure 4-21: DPIV velocity data for case fa15. Foil at maximum heave position. $S t_{T E}=0.55, \frac{2 A}{c}=1.0, \theta_{\circ}=30^{\circ}, \alpha_{\max }=24.8^{\circ}, \phi=90^{\circ}$. 
For large amplitude motions, the highest thrust cases ( $\mathrm{fa} 07$ and $\mathrm{fa} 12$ ) both occur at $S t_{A}=0.45$ so these are taken as the nominal cases from which the phase angle is varied. Two heave amplitudes are considered, $\frac{2 A}{c}=2.0$ and $\frac{2 A}{c}=1.0$, both with constant pitch amplitude $\theta_{\circ}=30^{\circ}$. The results for these are presented in Figures 4-23 through 4-28 and Figures 4-29 through 4-34 respectively. The trailing edge Strouhal number increases with increasing phase angle for a given heave and pitch amplitude. Hence, we expect the thrust to increase with increasing phase angle because the wake width increases. Conversely, when $\phi<90^{\circ}$, the angle of attack decreases with increasing phase angle which serves to lower the thrust generated. Above $90^{\circ}$, the angle of attack increases with increasing phase angle. These competing effects are not possible to separate with our apparatus.

Figure 4-22 illustrates the effect of phase change on the foil trajectory and angle of attack profile. When the phase angle is $90^{\circ}$, the foil pitch changes sign when the heave cycle reaches its maxima. The angle of attack profile is a flattened sinusoid with the peaks centered over the heave zero crossings. When the phase angle is less than $90^{\circ}$, the foil trajectory is toed-out, meaning that when the heave reaches its maxima, the foil points outward. The angle of attack profile peaks distort and the zero crossings occur before the heave maxima. Conversely, when the phase angle is greater than $90^{\circ}$, the trajectory is toed-in, and the $\alpha(t)$ zero crossings occur after the heave maxima. The shape of the angle of attack profile and zero crossings affect the location and strength of shed vorticity, and hence, should be influenced by the phase angle.

Figures 4-23 through 4-28 show the results for the large amplitude case. The $90^{\circ}$ phase case is shown in Figure 4-15. The phase angle clearly affects the development and evolution of the dynamic stall vortex. For low phase angles $\left(30\right.$ to $\left.50^{\circ}\right)$, the DSV forms and leaves the foil surface well before the position of maximum heave. This favors thrust development because the DSV contributes suction in the forward direction but is shed before it contributes to the drag when the foil pitch changes sign. As the phase angle is increased, the formation of the DSV is delayed further, causing the unfavorable condition of the DSV on the downstream side of the foil.

Figures 4-29 through 4-34 and 4-21 show the $\frac{2 A}{c}=1.0$ case. The same trend as in the higher amplitude case is observed: as the phase angle increases, the DSV development and shedding is delayed to a later point in the heave cycle. At the point of maximum heave, at $\phi=30^{\circ}$ the stall vortex is located approximately at mid-foil. As $\phi$ is increased to $90^{\circ}$, 


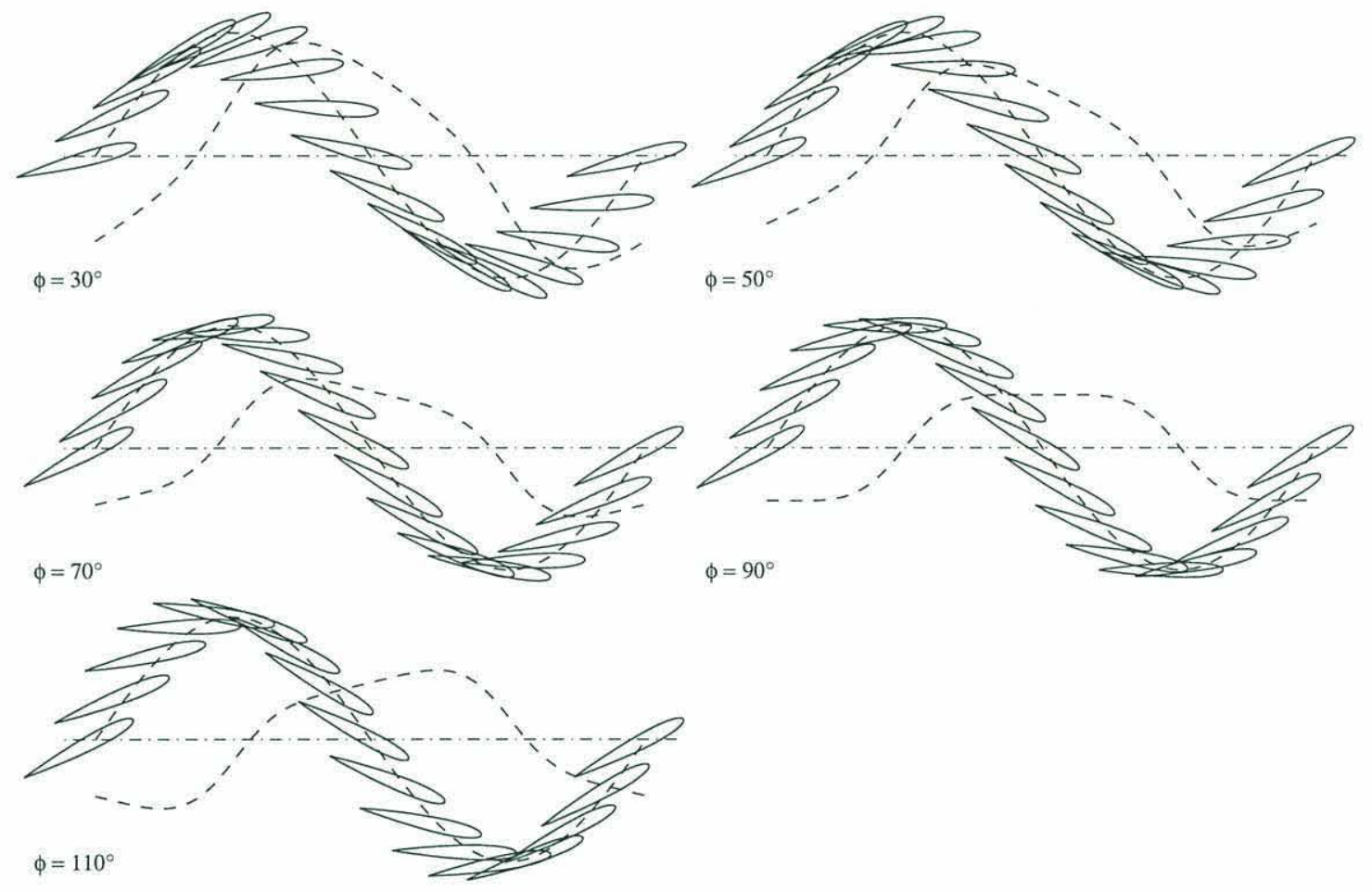

Figure 4-22: Foil trajectories and angle of attack phase relationship as a function of $\phi$ for $S t_{A}=0.45, \frac{2 A}{c}=2.0, \theta_{\circ}=30^{\circ}$. The dotted line indicates angle of attack relative magnitude and phase with respect to the motion. The phase angle affects both the angle of attack peak shape and zero crossings. 


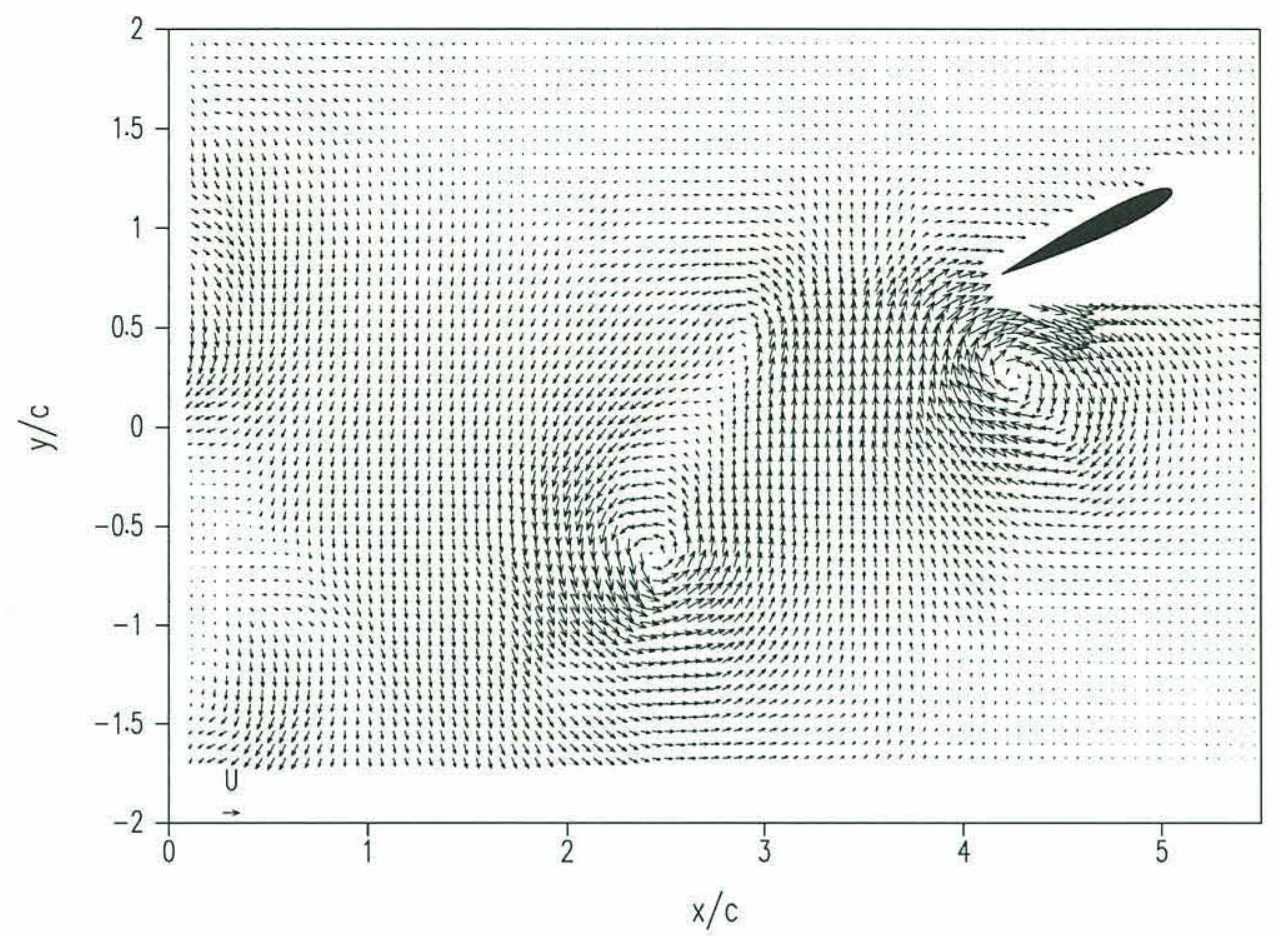

Figure 4-23: DPIV velocity data for case fa19. Foil at maximum heave position. $S t_{T E}=0.33, \frac{2 A}{c}=2.0, \theta_{\circ}=30^{\circ}, \alpha_{\max }=52.7^{\circ}, \phi=30^{\circ}$.

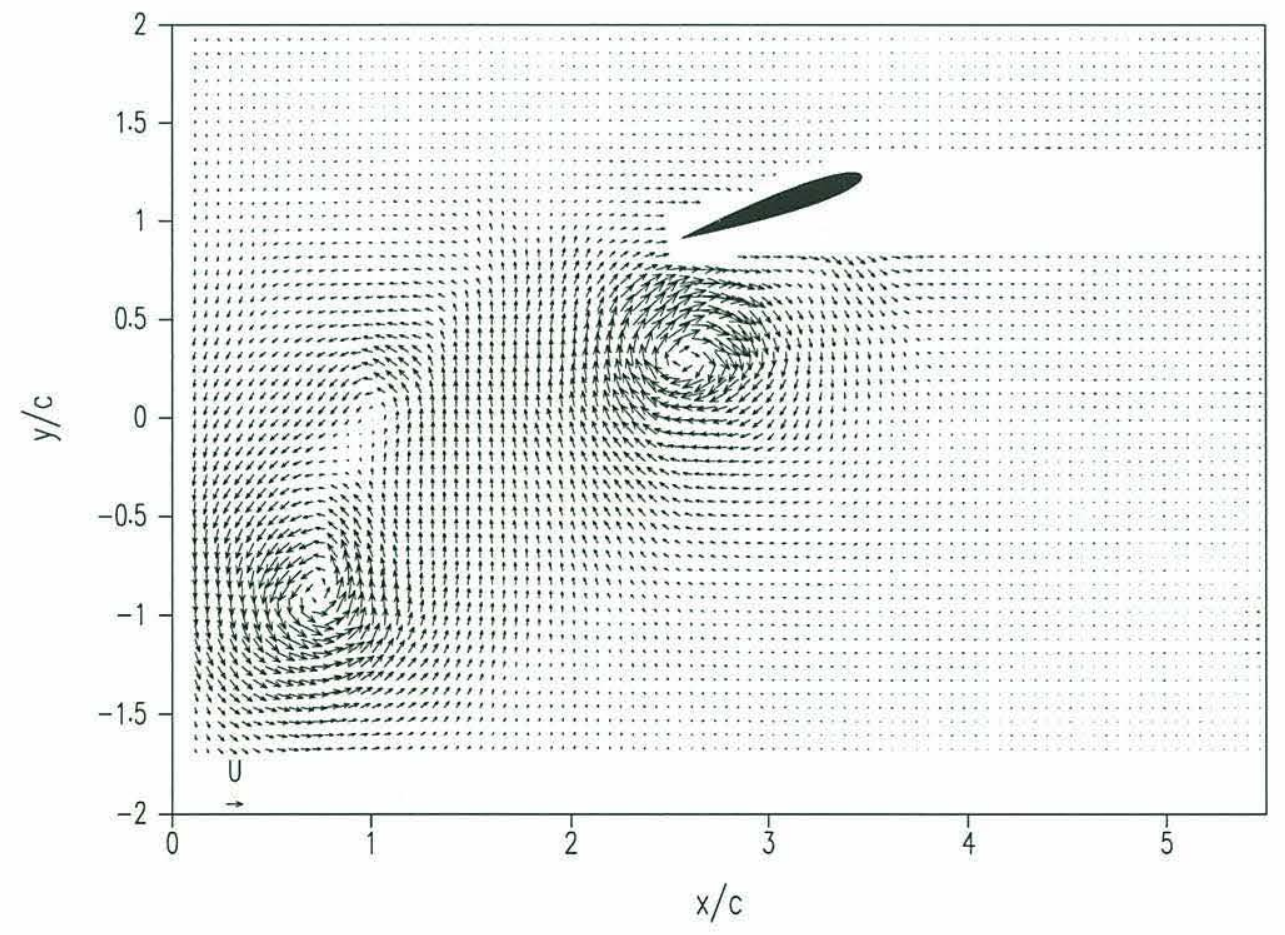

Figure 4-24: DPIV velocity data for case fa20. Foil at maximum heave position. $S t_{T E}=0.37, \frac{2 A}{c}=2.0, \theta_{\circ}=30^{\circ}, \alpha_{\max }=42.4^{\circ}, \phi=50^{\circ}$. 


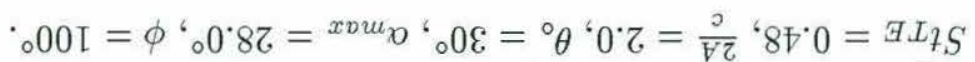

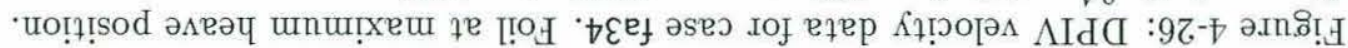

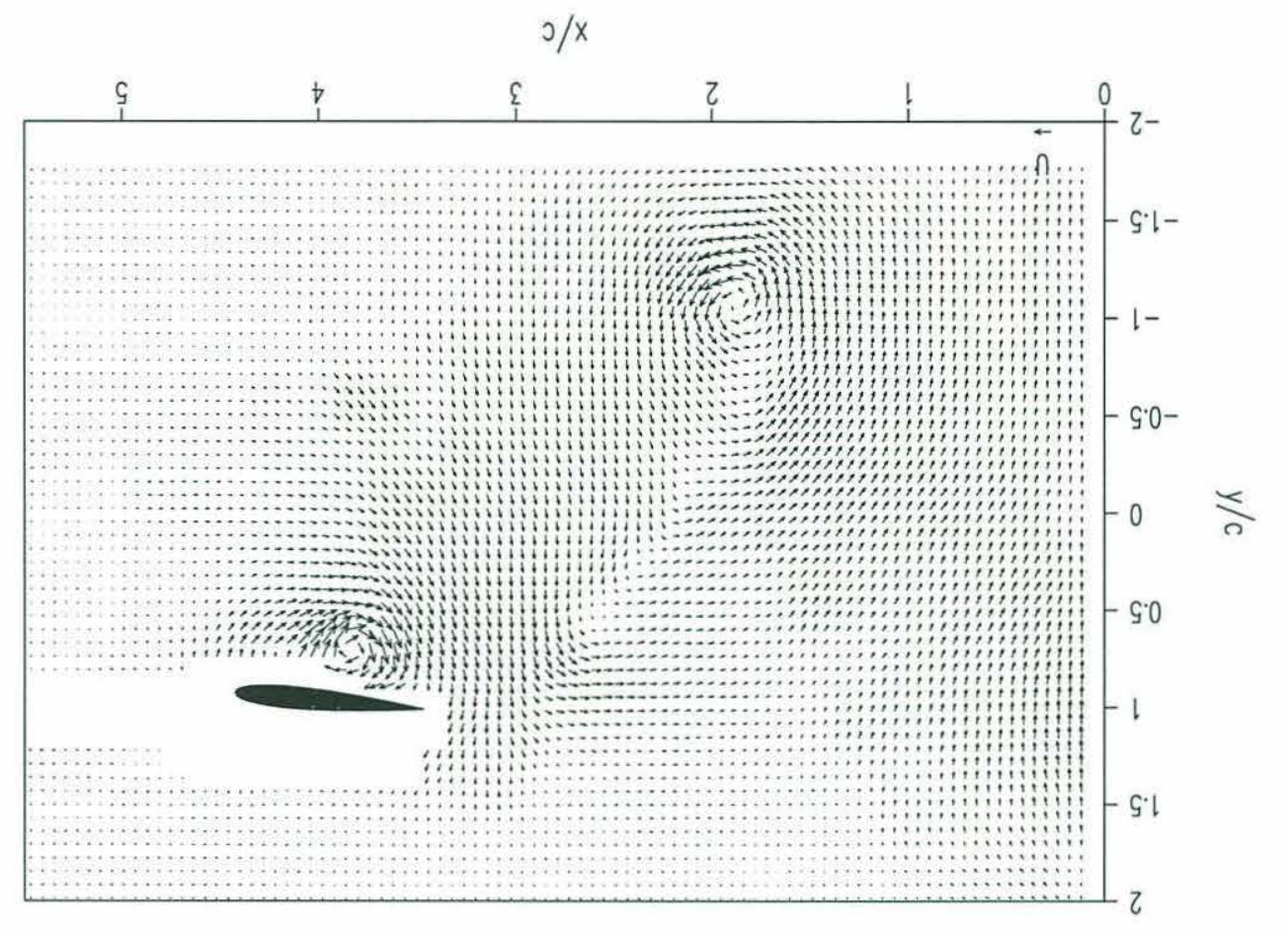

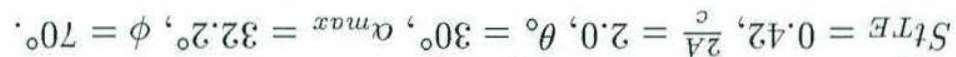

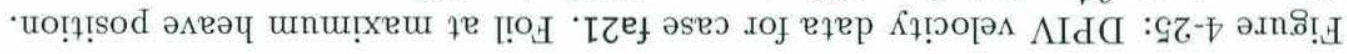

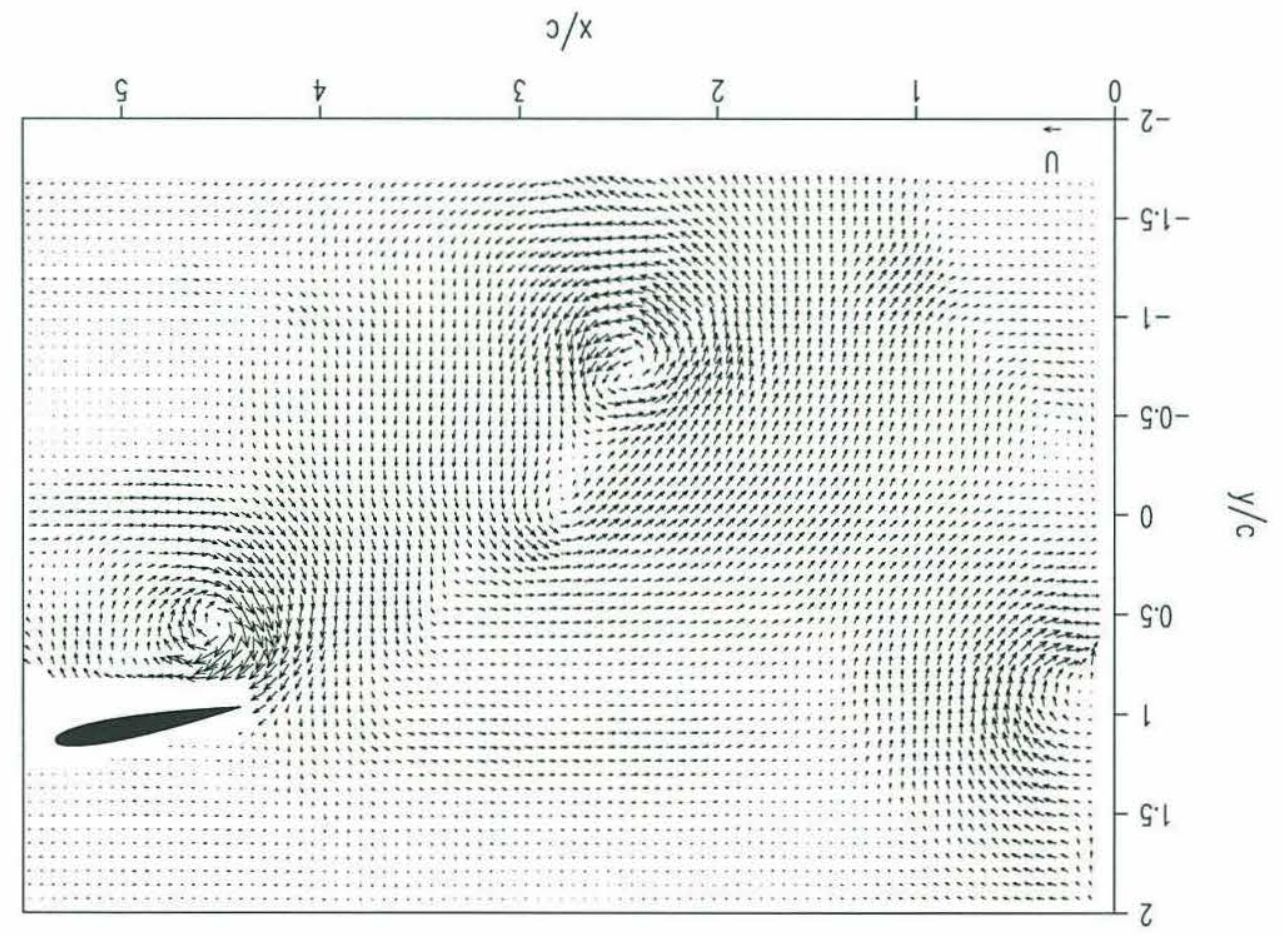




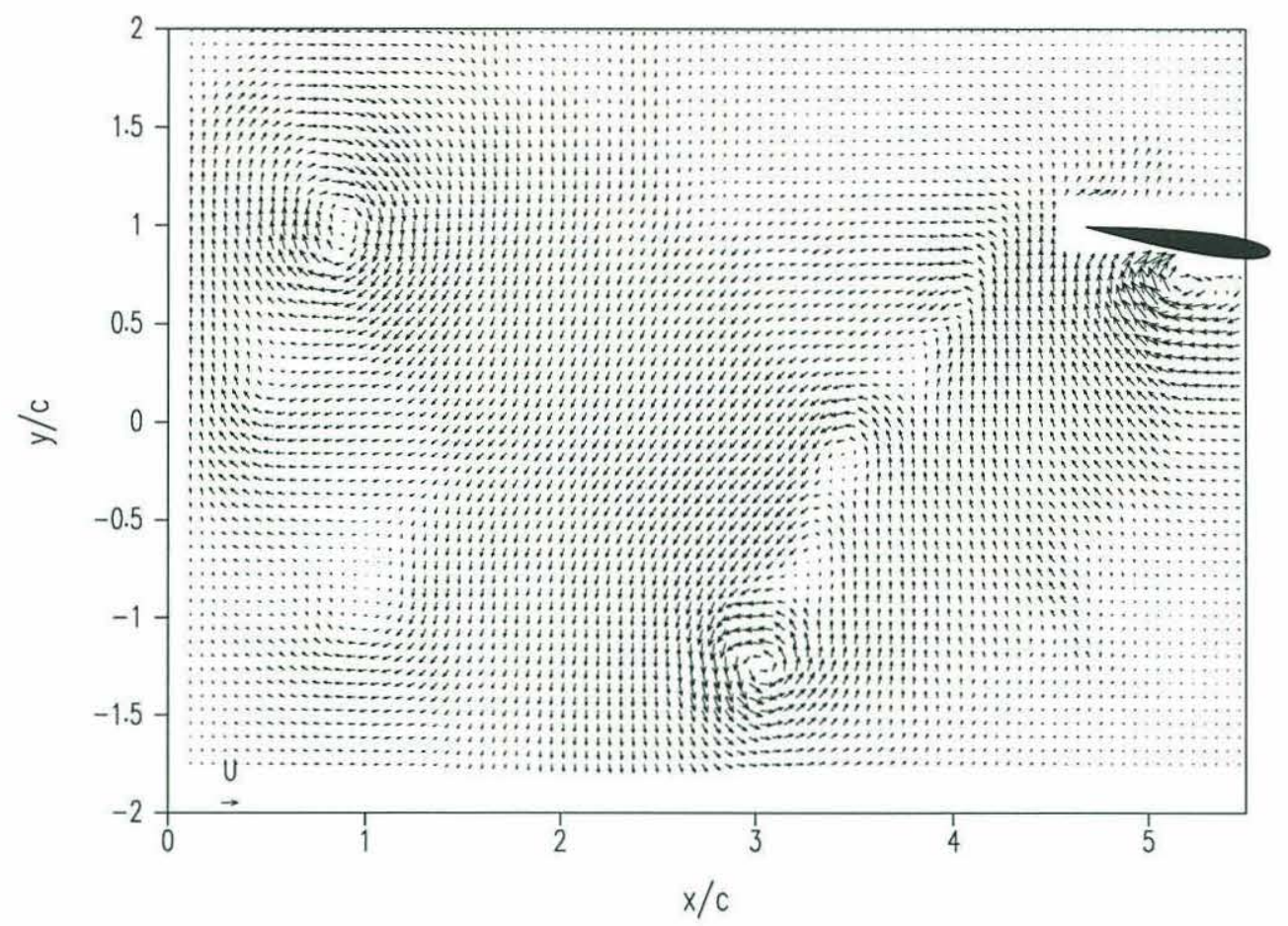

Figure 4-27: DPIV velocity data for case fa33. Foil at maximum heave position. $S t_{T E}=0.48, \frac{2 A}{c}=2.0, \theta_{\circ}=30^{\circ}, \alpha_{\max }=30.0^{\circ}, \phi=105^{\circ}$.

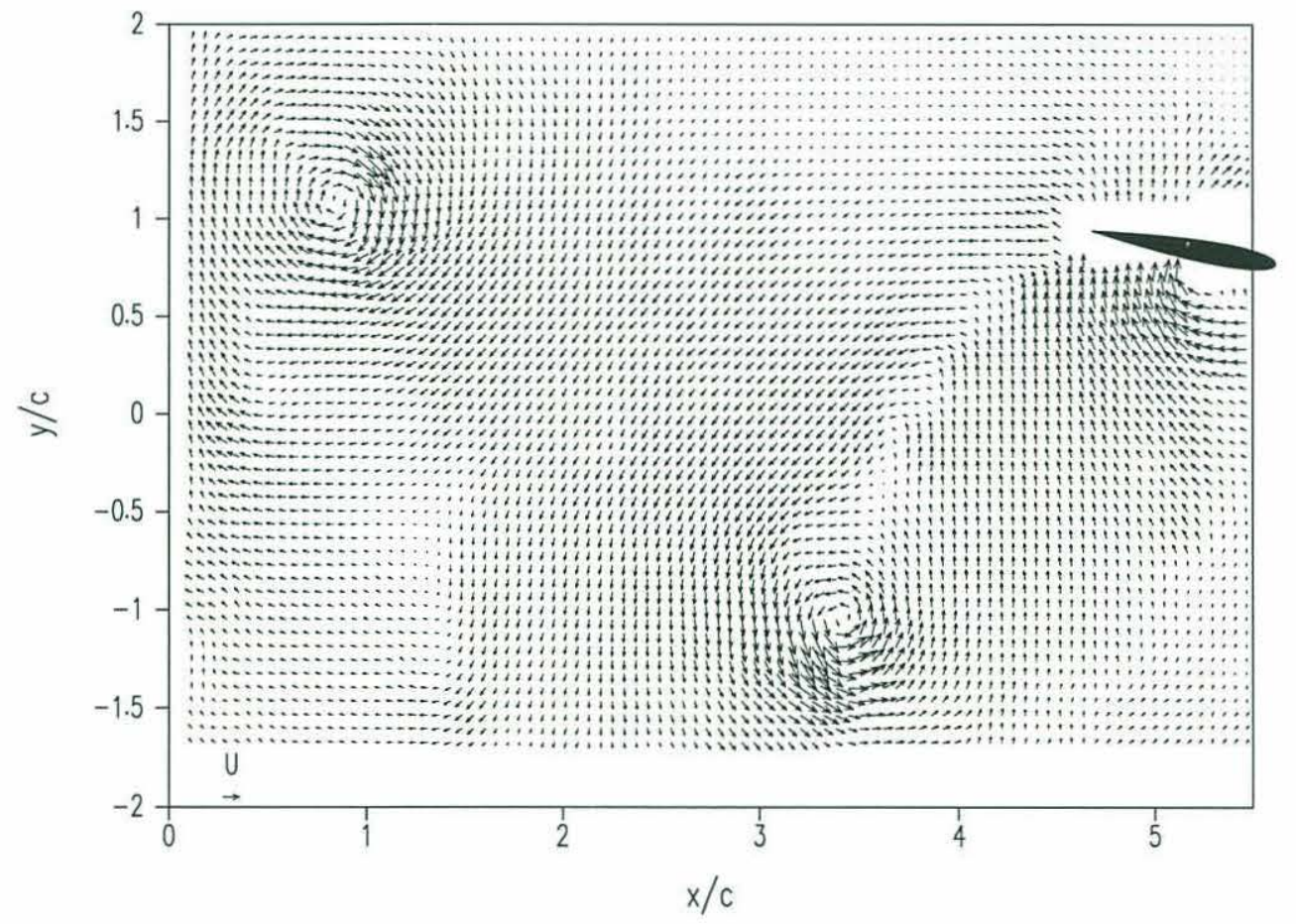

Figure 4-28: DPIV velocity data for case fa22. Foil at maximum heave position. $S t_{T E}=0.52, \frac{2 A}{c}=2.0, \theta_{\circ}=30^{\circ}, \alpha_{\max }=32.2^{\circ}, \phi=110^{\circ}$. 


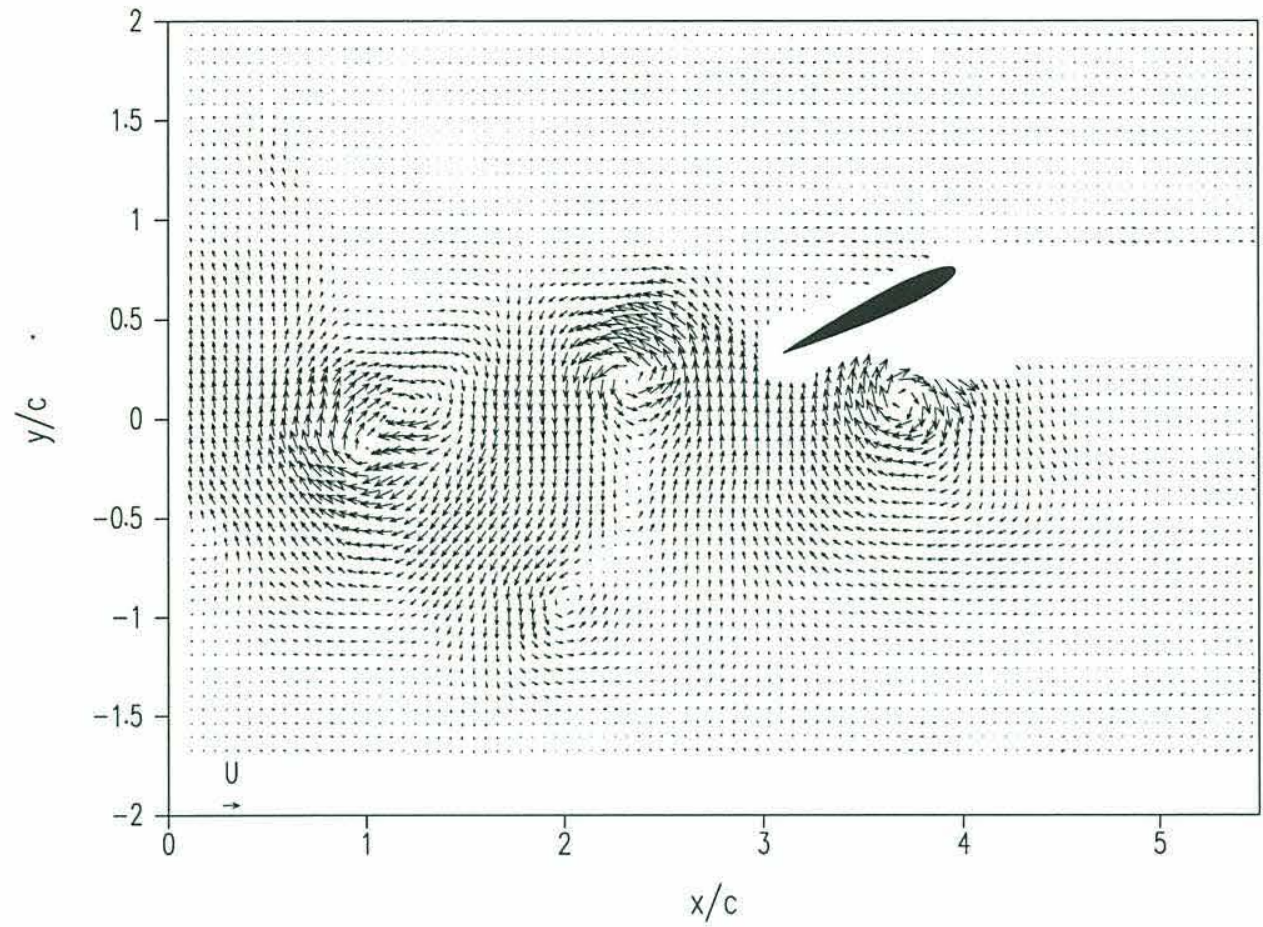

Figure 4-29: DPIV velocity data for case fa23. Foil at maximum heave position. $S t_{T E}=0.24, \frac{2 A}{c}=1.0, \theta_{\circ}=30^{\circ}, \alpha_{\max }=52.7^{\circ}, \phi=30^{\circ}$.

the stall vortex is beginning to form near the nose. At higher phase angles, the stall vortex does not form until after the point of maximum heave. Again, this delay of stall acts to diminish the average thrust.

The $\frac{2 A}{c}=1.0$ case wakes are much more mixed than the larger amplitude case, especially for $\phi>90^{\circ}$. This happens because the same signed DSV and trailing edge vortices do not merge well in the near wake. Increasing the phase angle delays the shedding of the DSV enough that constructive merging with trailing edge generated vorticity is not synchronized. The resultant wake is comprised of stretched and distorted pairs which broaden and mix the wake.

\subsubsection{Group IX}

In group IX, we again vary the phase angle while holding other kinematic variables constant. The heave amplitude, pitch angle and Strouhal number coincide exactly with the values used in the M.I.T. experiments for the highest efficiency achieved. The purpose of these runs is to ascertain the flow mechanisms which influence thrust and efficiency variations by direct comparison to the M.I.T. experiments. 


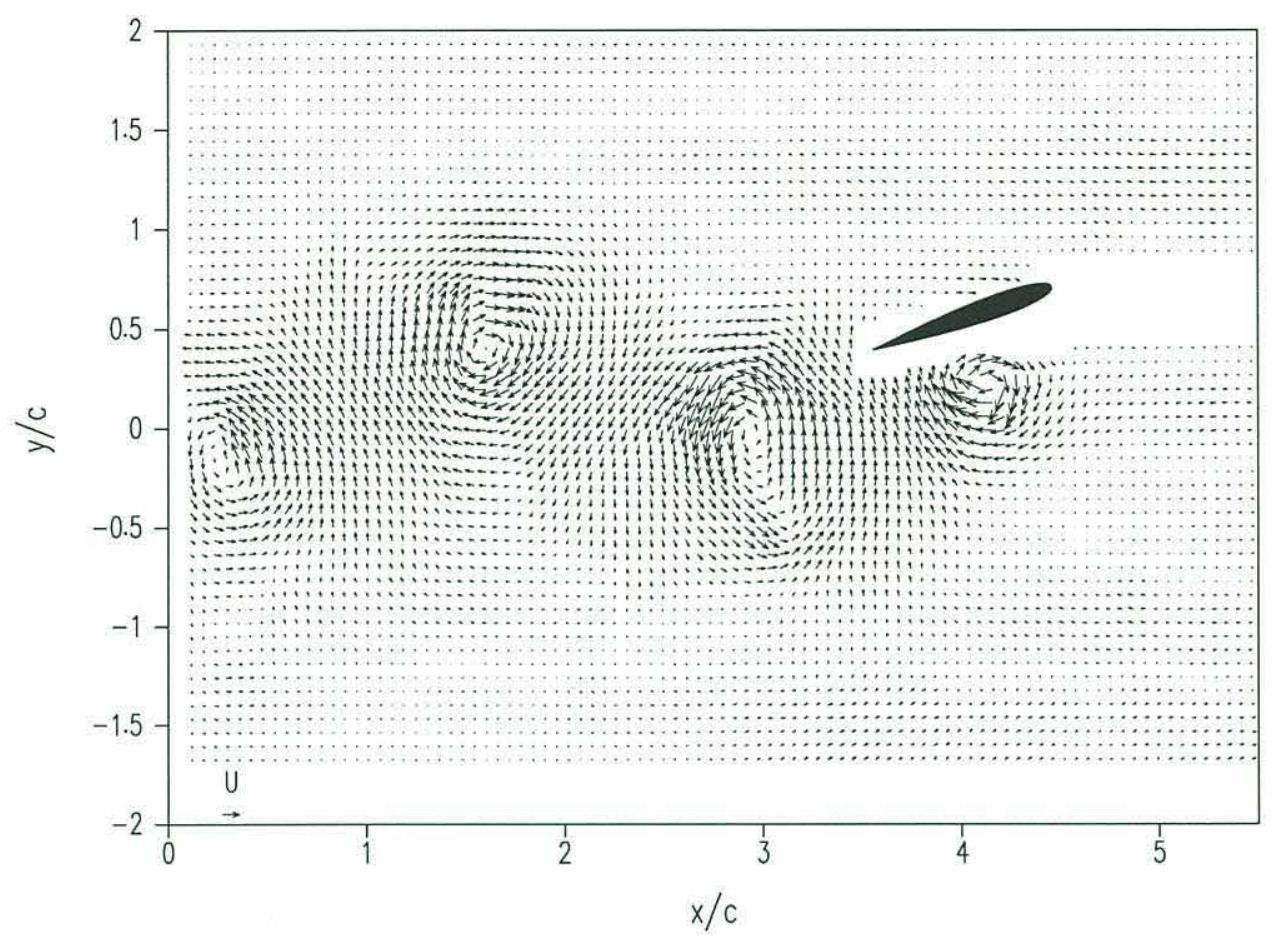

Figure 4-30: DPIV velocity data for case fa24. Foil at maximum heave position. $S t_{T E}=0.35, \frac{2 A}{c}=1.0, \theta_{\circ}=50^{\circ}, \alpha_{\max }=42.4^{\circ}, \phi=50^{\circ}$.

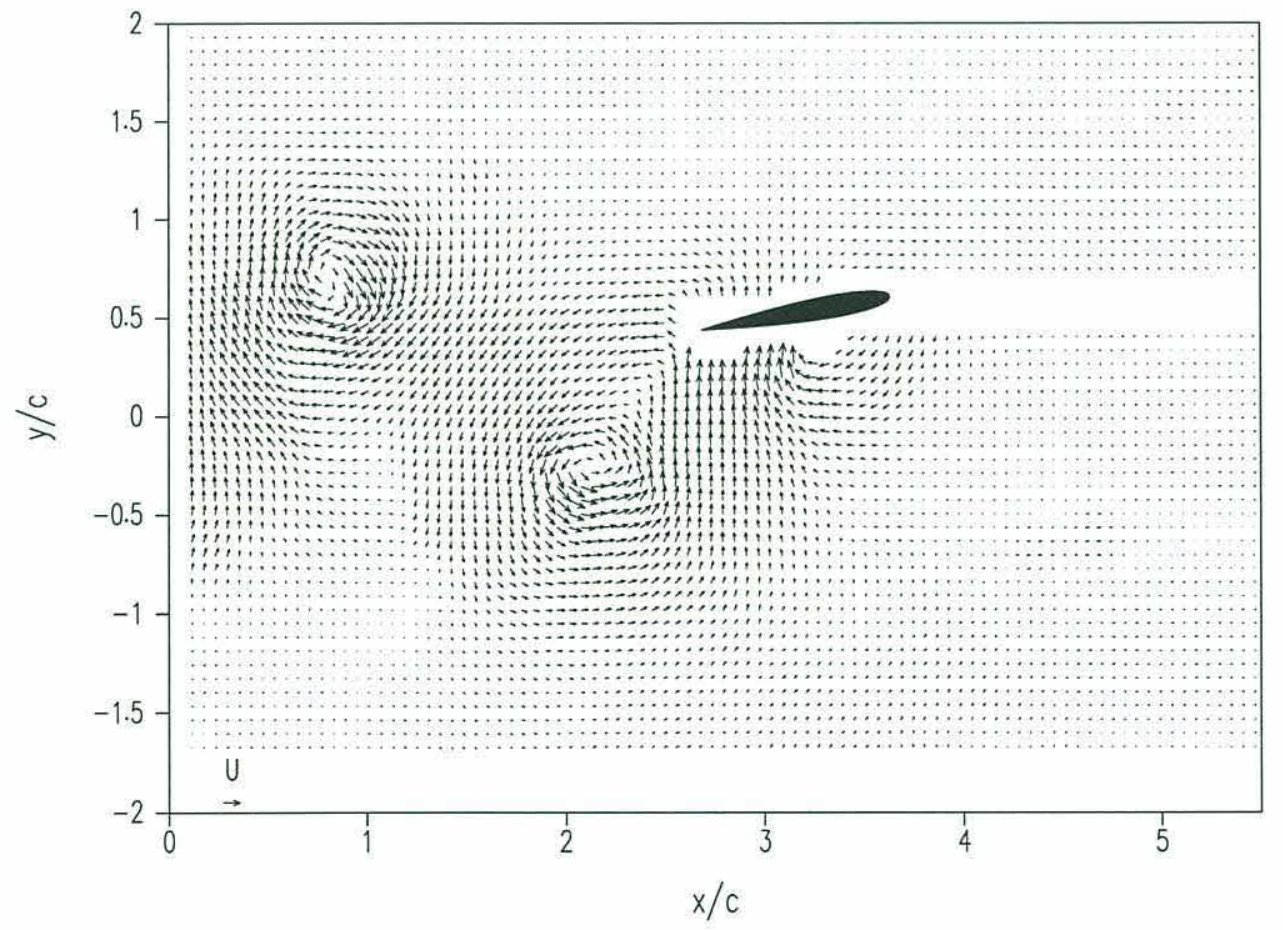

Figure 4-31: DPIV velocity data for case fa25. Foil at maximum heave position. $S t_{T E}=0.45, \frac{2 A}{c}=1.0, \theta_{\circ}=30^{\circ}, \alpha_{\max }=32.2^{\circ}, \phi=70^{\circ}$. 


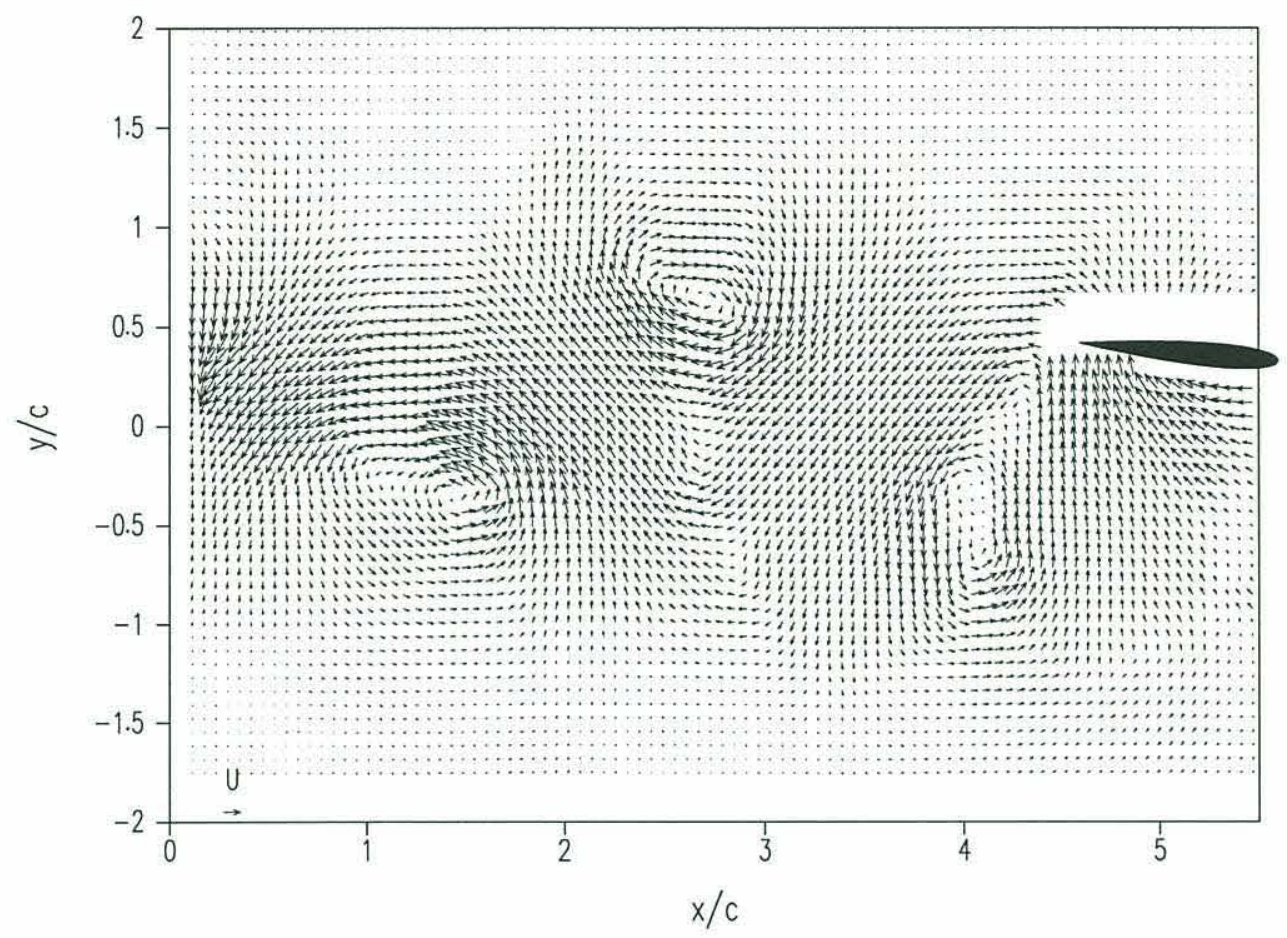

Figure 4-32: DPIV velocity data for case fa30. Foil at maximum heave position. $S t_{T E}=0.55, \frac{2 A}{c}=1.0, \theta_{\circ}=30^{\circ}, \alpha_{\max }=28.0^{\circ}, \phi=100^{\circ}$.

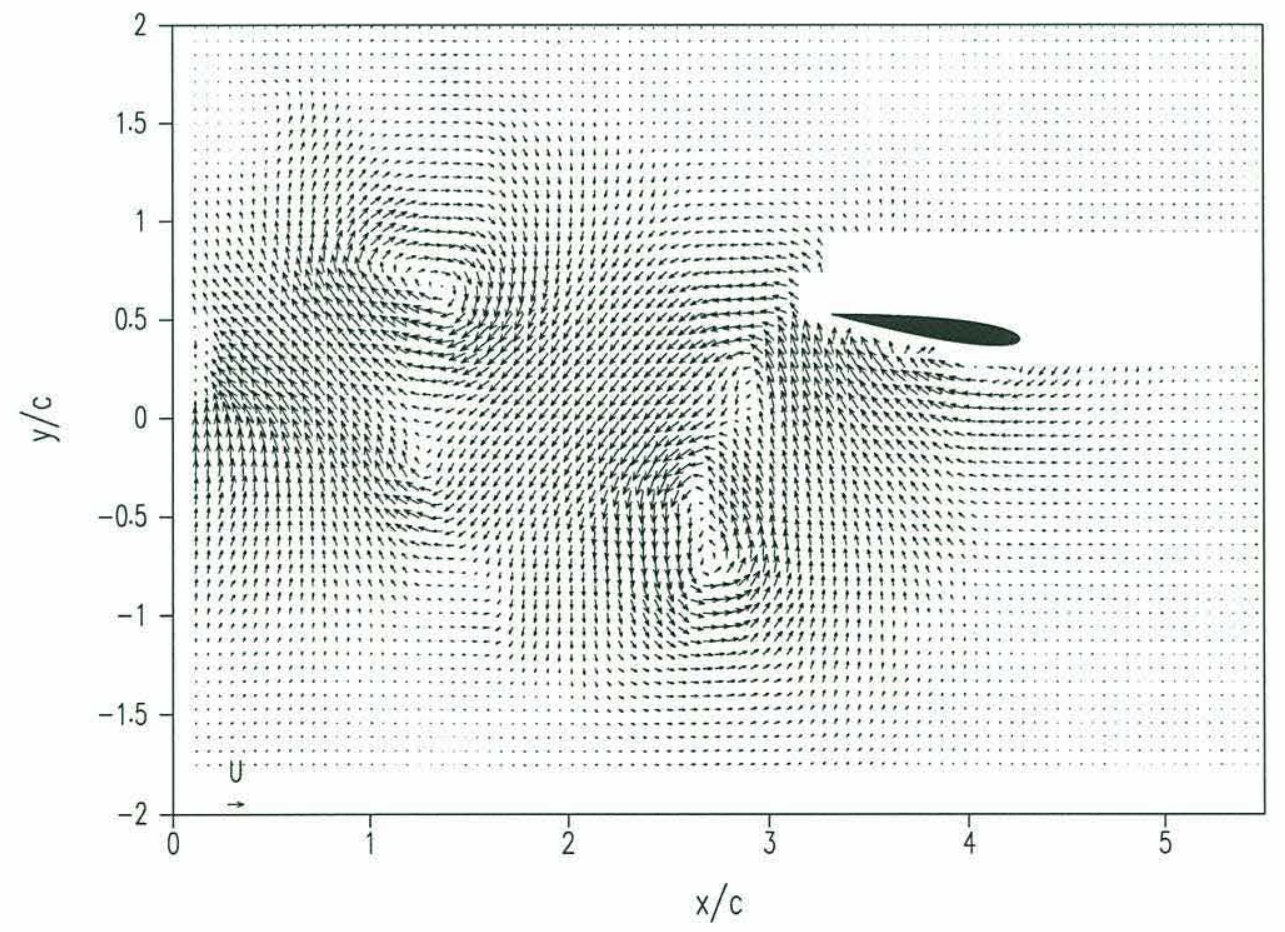

Figure 4-33: DPIV velocity data for case fa32. Foil at maximum heave position. $S t_{T E}=0.55, \frac{2 A}{c}=1.0, \theta_{\circ}=30^{\circ}, \alpha_{\max }=30.0^{\circ}, \phi=105^{\circ}$. 


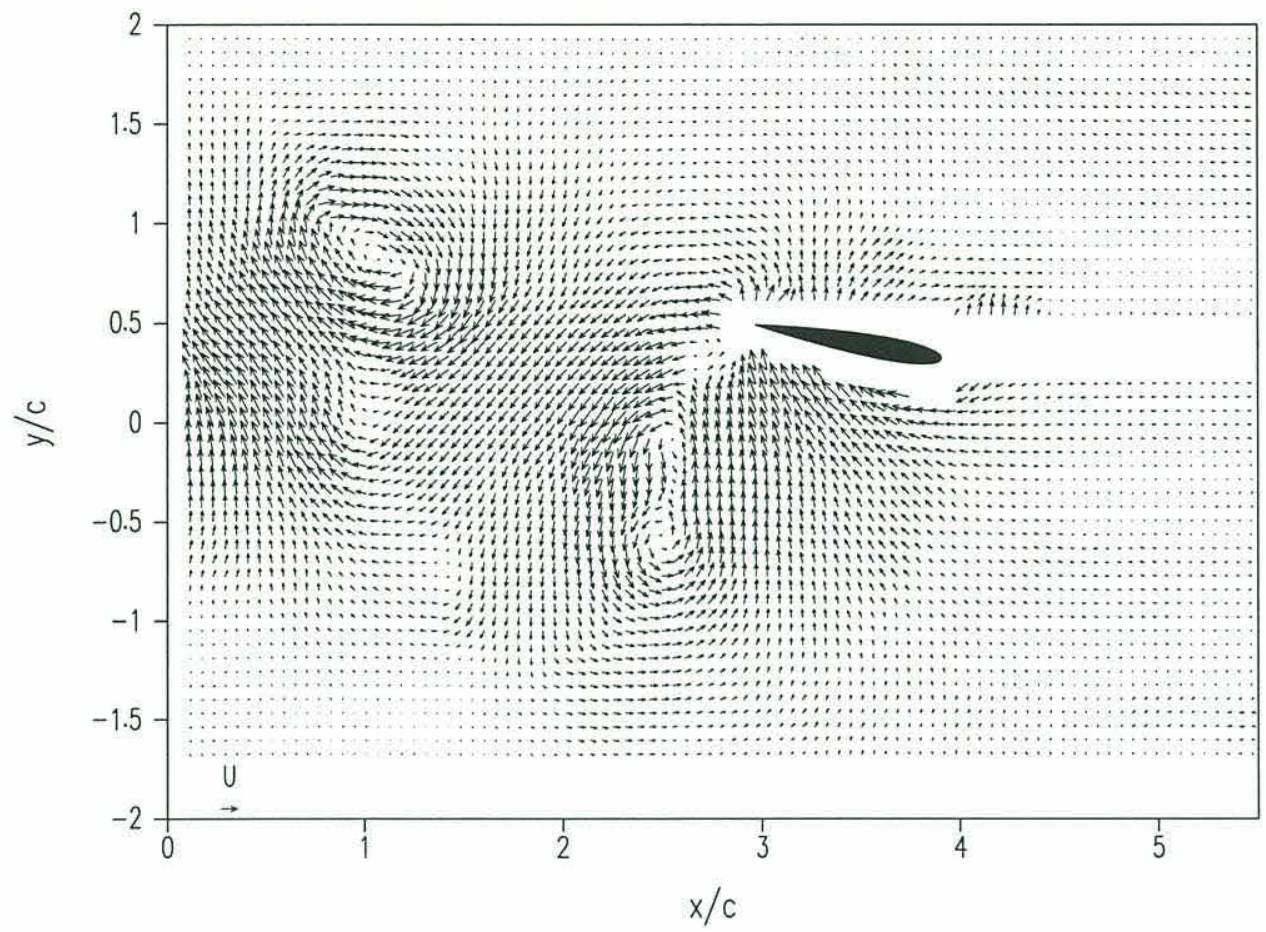

Figure 4-34: DPIV velocity data for case fa26. Foil at maximum heave position. $S t_{T E}=0.62, \frac{2 A}{c}=1.0, \theta_{\circ}=30^{\circ}, \alpha_{\max }=32.2^{\circ}, \phi=110^{\circ}$.

Figures 4-35 through 4-37 show the results for group IX. At $\phi=90^{\circ}$ (Figure 4-35), the leading edge vortex is not yet well formed when the foil reaches the point of maximum heave. For $\phi=75^{\circ}$ (Figure 4-36), the leading edge vortex forms much earlier and is well defined at the point of maximum heave. When $\phi=105^{\circ}$ (Figure 4-37), dynamic stall is delayed until well after the point of maximum heave.

To further explore the development of dynamic stall, the group IX experiments were repeated with the flow illuminated from the side which revealed the stall process without any shadows or obstructions. Figure 4-38 shows a sequence of velocity fields obtained for the case fa36 for roughly half an oscillation cycle. As noted in the previous results, the leading edge vortex does not organize until the foil is in its maximum heave position $\left(t^{*}=\frac{t}{T}=0.25\right)$ when the pitch angle is zero. At this time, the stall vortex is roughly the same size as the foil. The DSV concentrates into a smaller, more organized vortex just before leaving the trailing edge at $t^{*}=0.335$.

Figure 4-39 shows the fa37 case where the phase angle is reduced to $75^{\circ}$. Clearly, the dynamic stall proceeds more rapidly than in the $90^{\circ}$ case. The DSV concentrates immediately after forming compared to the sluggishly organized bubble in the previous case. The DSV leaves the foil at $t^{*}=0.293$, just as the foil pitch changes sign. The 


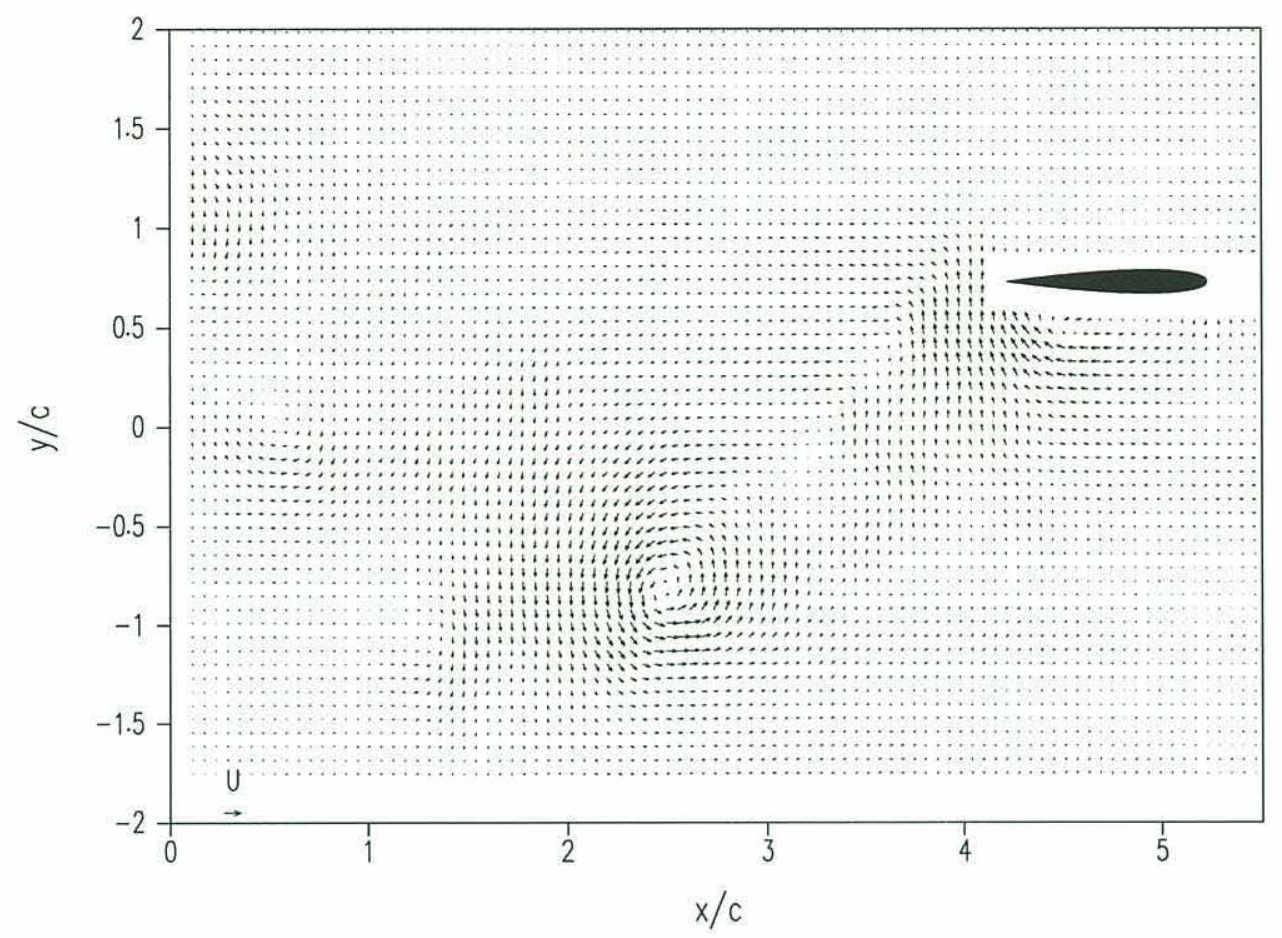

Figure 4-35: DPIV velocity data for case fa36. Foil at maximum heave position. $S t_{T E}=$ $0.35, \frac{2 A}{c}=1.5, \theta_{\circ}=30^{\circ}, \alpha_{\max }=15^{\circ}, \phi=90^{\circ}$.

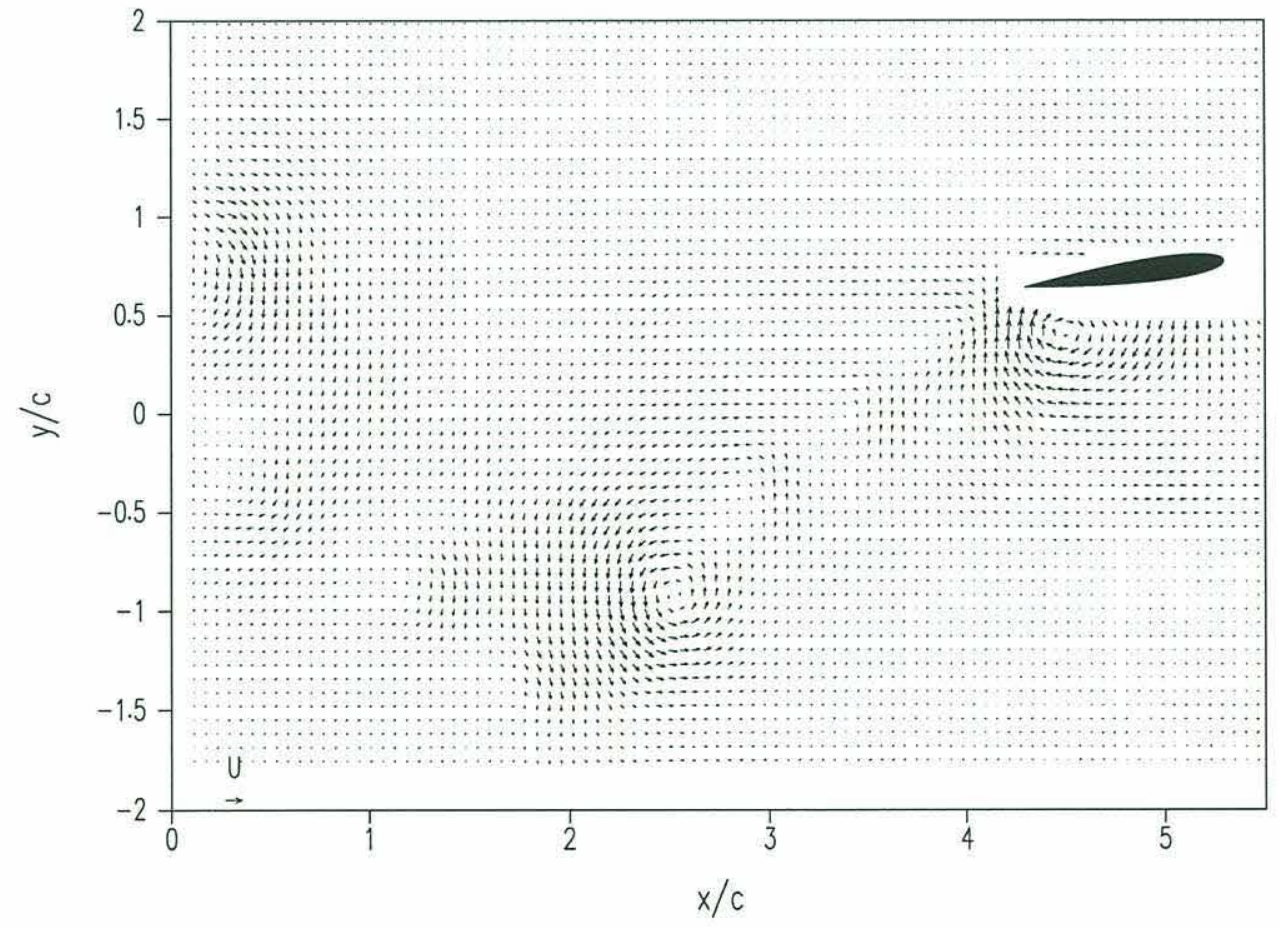

Figure 4-36: DPIV velocity data for case fa37. Foil at maximum heave position. $S t_{T E}=$ $0.31, \frac{2 A}{c}=1.5, \theta_{\circ}=30^{\circ}, \alpha_{\max }=20.2^{\circ}, \phi=75^{\circ}$. 


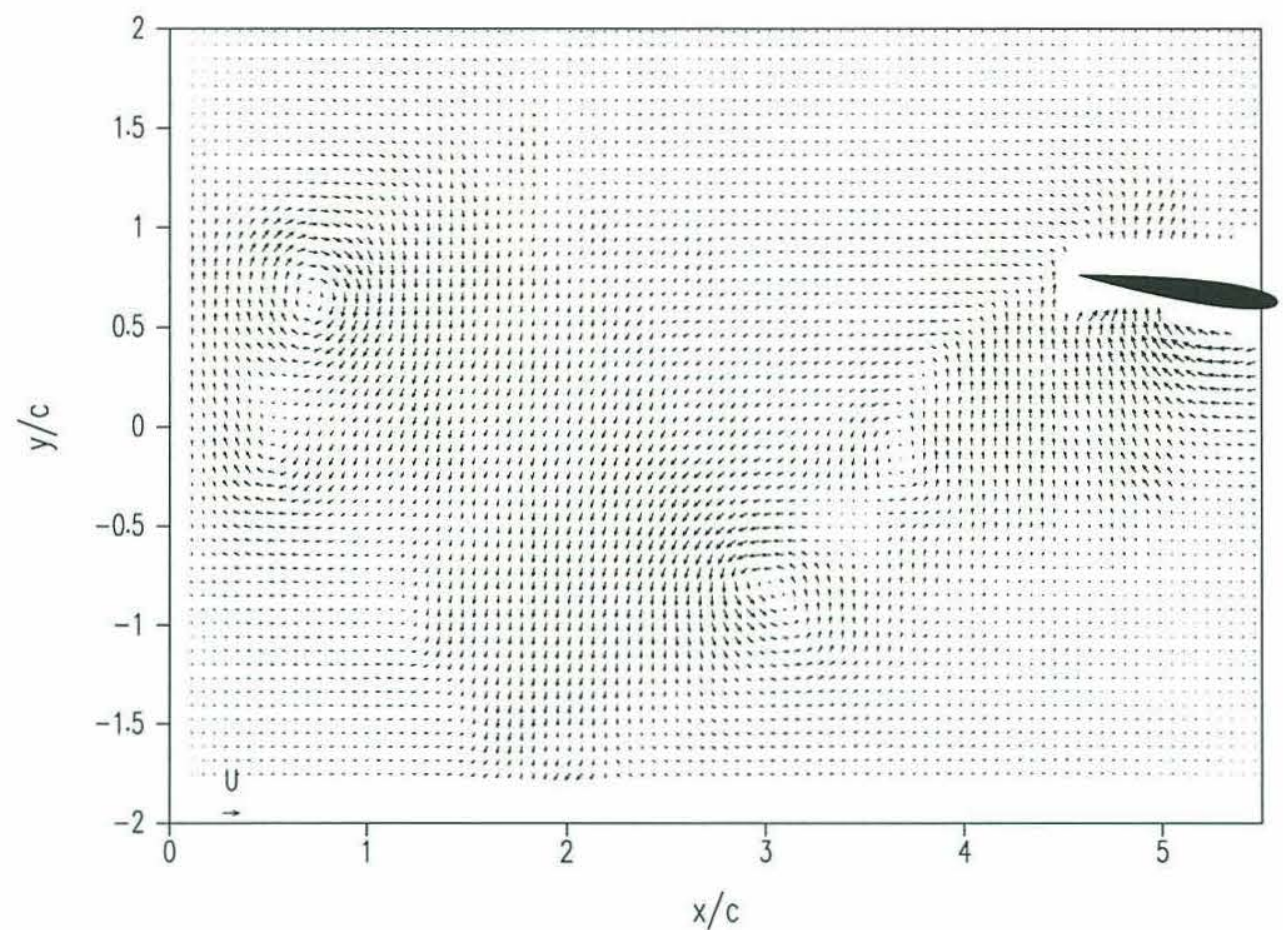

Figure 4-37: DPIV velocity data for case fa38. Foil at maximum heave position. $S t_{T E}=$ $0.38, \frac{2 A}{c}=1.5, \theta_{\circ}=30^{\circ}, \alpha_{\max }=20.2^{\circ}, \phi=105^{\circ}$.

dynamics of the stall process in this case are clearly favorable compared to the $\phi=90^{\circ}$ case. The well concentrated DSV is located such that it provides suction to contribute to the upstream thrust force and the vortex is shed before its suction contributes to the drag force.

Figure 4-40 shows the temporal development of the fa38 case where the phase angle is increased to $105^{\circ}$. The DSV development is very similar to the $\phi=90^{\circ}$ case, except that now, the DSV forms and departs the foil a little later in the cycle. The small increase in the angle of attack is evidenced by the more energetic and concentrated DSV. For instance, at the point of maximum heave ( $\approx$ frame 7 in Figures 4-38 and 4-40), the DSV occupies the entire foil surface in the $90^{\circ}$ case but only about a third of the foil surface in the $105^{\circ}$ case. Here, the evolution of the DSV is unfavorable to the overall thrust as the separation of the vortex from the foil is delayed until $t^{*}=0.377$. 

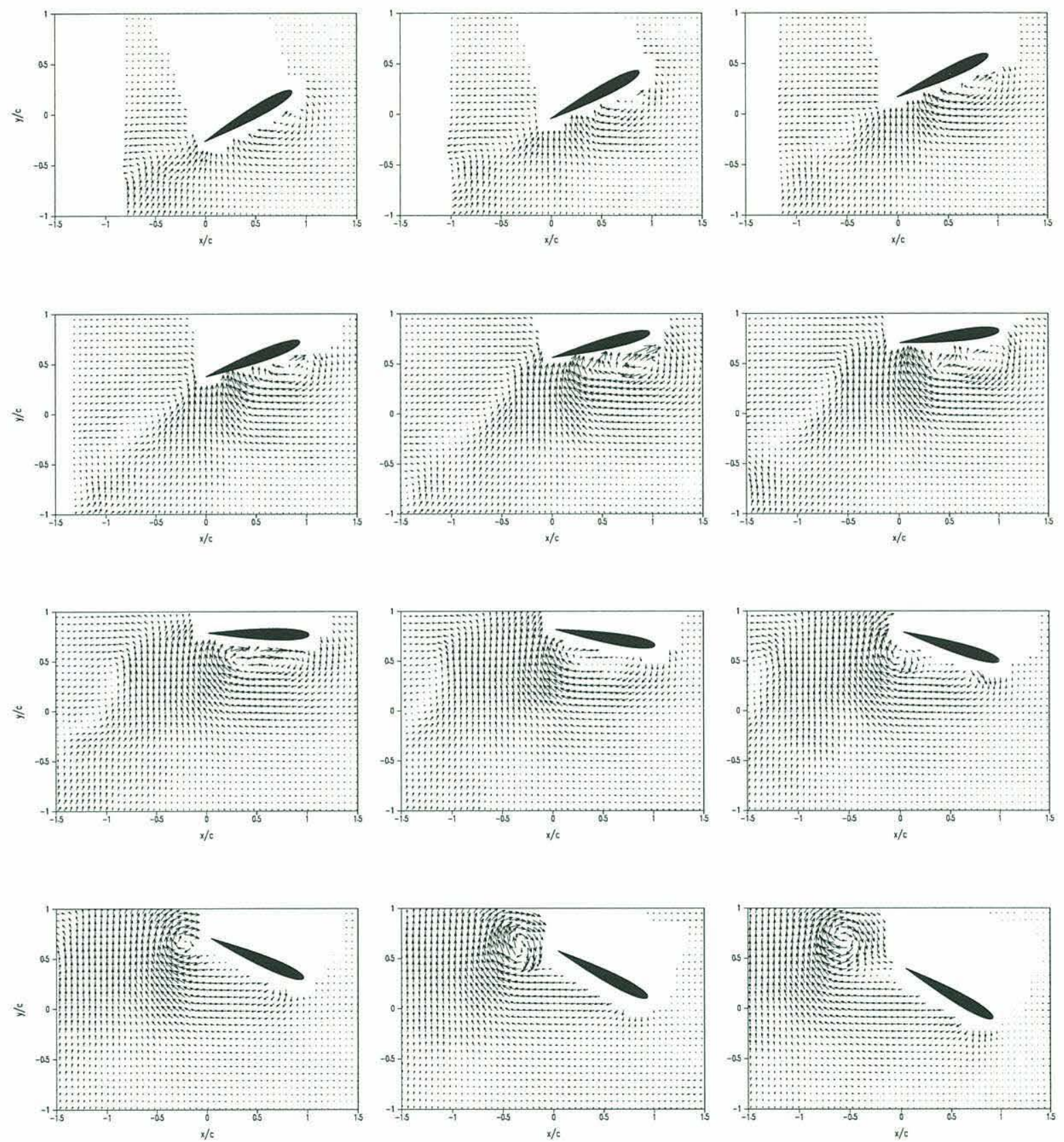

Figure 4-38: Velocity field sequence for fa36, $S t_{T E}=0.32, \frac{2 A}{c}=1.5, \theta_{\circ}=30^{\circ}, \phi=90^{\circ}$. Sequence starts in upper left corner at $t^{*}=\frac{t}{T}=0$, proceeds from left to right, top to bottom in increments $\Delta t^{*}=.042$. The $x$-axis is adjusted such that the trailing edge is located at $x=0$. 

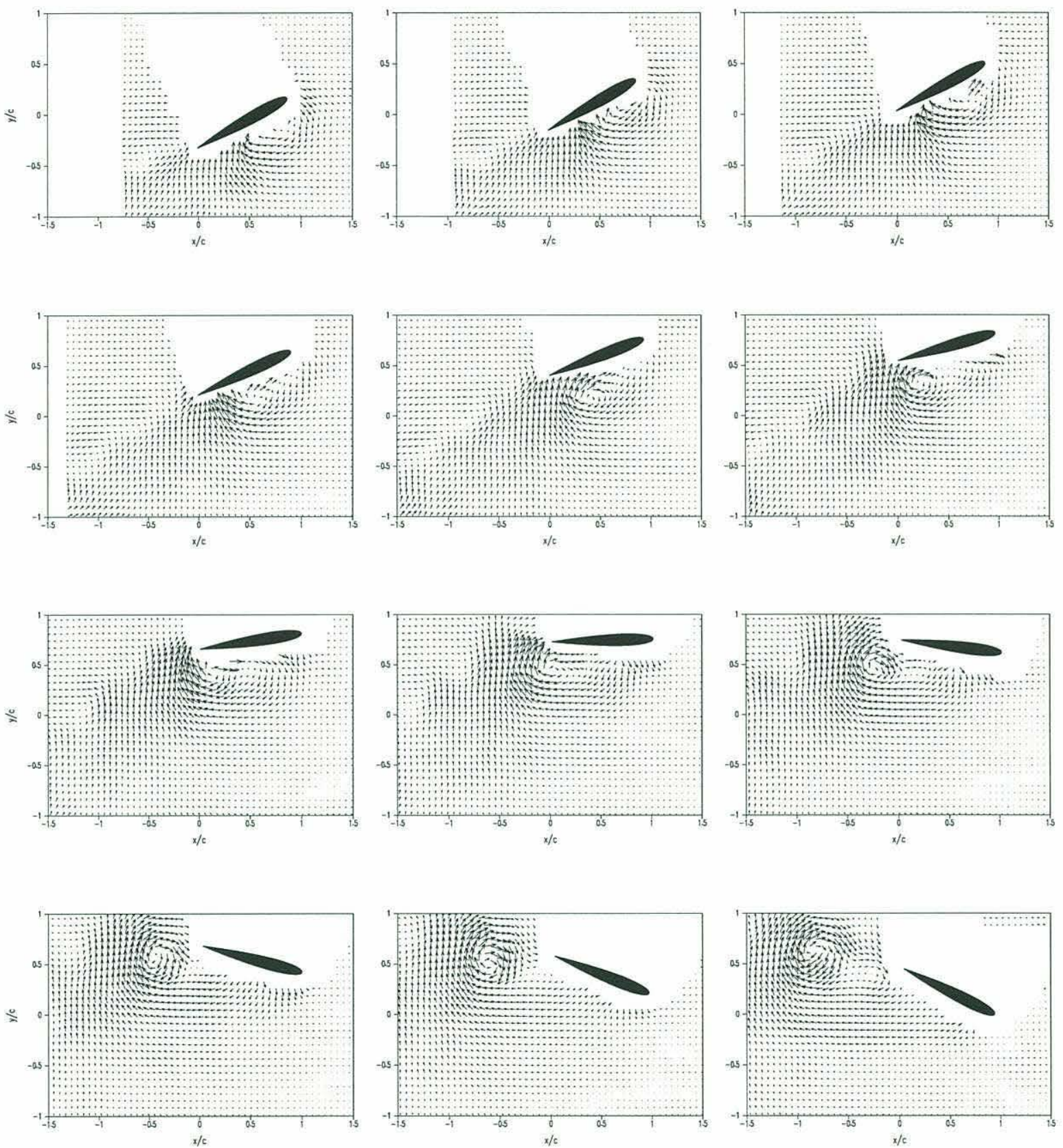

Figure 4-39: Velocity field sequence for fa37, $S t_{T E}=0.32, \frac{2 A}{c}=1.5, \theta_{\circ}=30^{\circ}, \phi=75^{\circ}$. Sequence starts in upper left corner at $t^{*}=\frac{t}{T}=0$, proceeds from left to right, top to bottom in increments $\Delta t^{*}=.042$. 

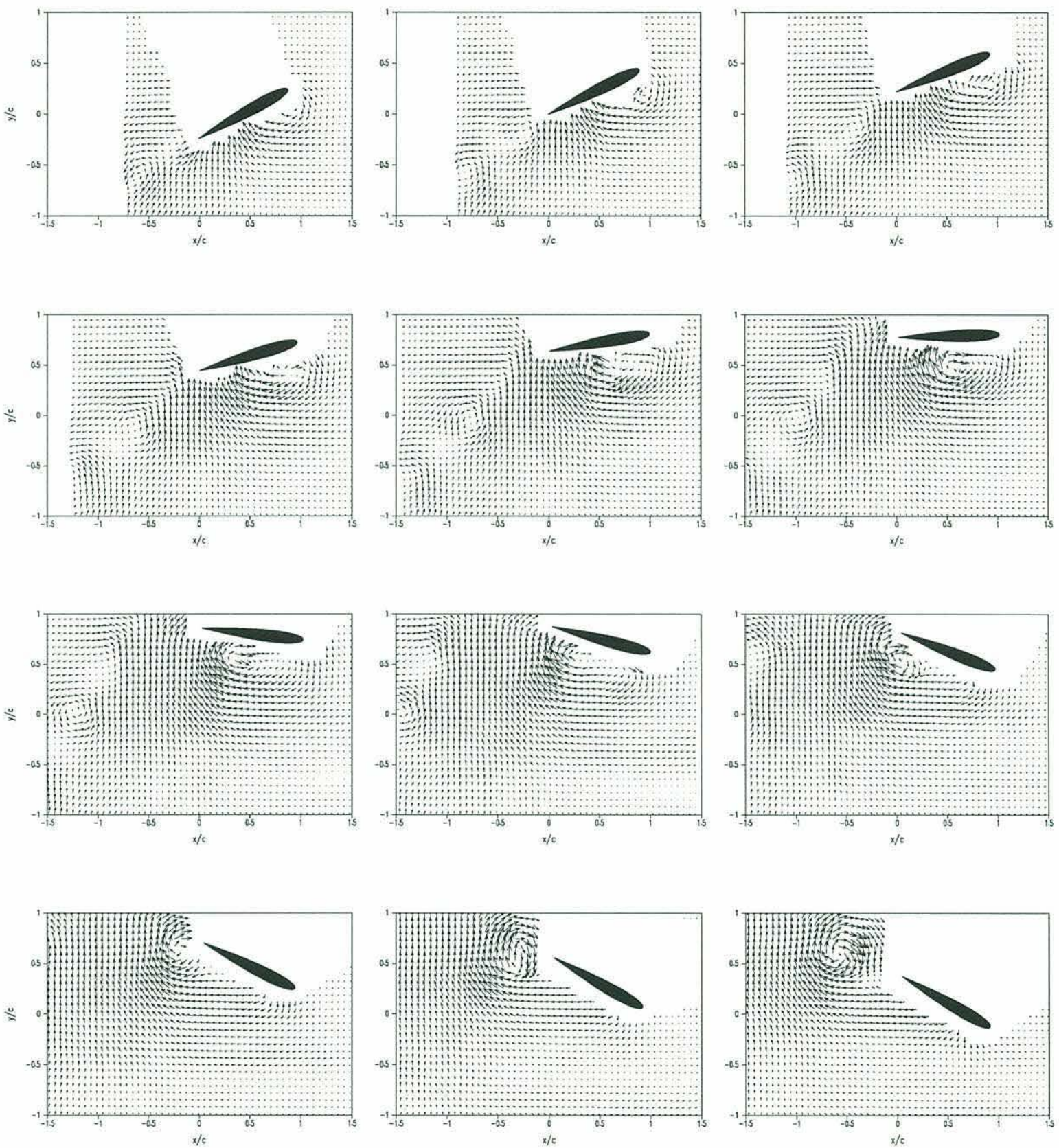

Figure 4-40: Velocity field sequence for fa38, $S t_{T E}=0.32, \frac{2 A}{c}=1.5, \theta_{\circ}=30^{\circ}, \phi=105^{\circ}$. Sequence starts in upper left corner at $t^{*}=\frac{t}{T}=0$, proceeds from left to right, top to bottom in increments $\Delta t^{*}=.042$. 


\subsection{Discussion}

\subsubsection{Effect of phase angle}

Figure 4-41 summarizes the force coefficient variation with phase angle for groups VII and VIII. Figure 4-42 shows the corresponding wake width variation. In both amplitude cases, the force coefficient increases with increasing phase angle up to $90^{\circ}$. Above $\phi=90^{\circ}$, there is a moderate decrease in the force for $\frac{2 A}{c}=2.0$ and a marked $72 \%$ increase for $\frac{2 A}{c}=1.0$. The drop in force coefficient at $\phi=100^{\circ}$ and $\frac{2 A}{c}=2.0$ was verified by repeating that case. The same anomalous result was obtained. With respect to kinematics, there are two competing force contributing factors which vary with the phase angle. First, the trailing edge Strouhal number increases with increasing phase angle. Also, up to $\phi=90^{\circ}$, the angle of attack decreases with increasing phase, and after $90^{\circ}$, it increases.

The wake size grows nearly linearly with phase for both the $\frac{2 A}{c}=1.0$ and 2.0 cases. Interestingly, the $\frac{2 A}{c}=1.0$ case achieves higher thrust for $\phi>90^{\circ}$ compared to the 2.0 case, yet has a much narrower wake. The lower amplitude case jet is more concentrated near the centerline and more energetic.

The rate of dynamic stall development depends on the phase angle. At high phase angles, leading edge separation is delayed later in the cycle. Figure $4-43$ shows this by plotting the position of the DSV on the foil surface at the heave maximum for the three amplitudes studied. If the DSV sheds before the foil pitches downward, the stall vortex will only contribute to thrust, not drag. If the DSV develops later, it will remain attached to the foil during the downstroke and thus add to the drag of the foil. The best performance occurs for $70^{\circ}<\phi<90^{\circ}$ because the DSV is favorably positioned and the thrust coefficient is high. Phase angle has greater influence over the DSV position in the higher amplitude cases.

\subsubsection{Thrust force}

Figure 4-44 shows how the momentum analysis force coefficients compare with the M.I.T. data from Figure 4-1 for the $90^{\circ}$ phase angle cases. Results for all amplitudes are plotted although the M.I.T. data are for $\frac{2 A}{c}=1.5$ only. Although meant to be a general comparison due to the difference in amplitudes, our force coefficients are the same order of magnitude as the M.I.T. data and reflect the same trends across the parameter space. The force coefficient 


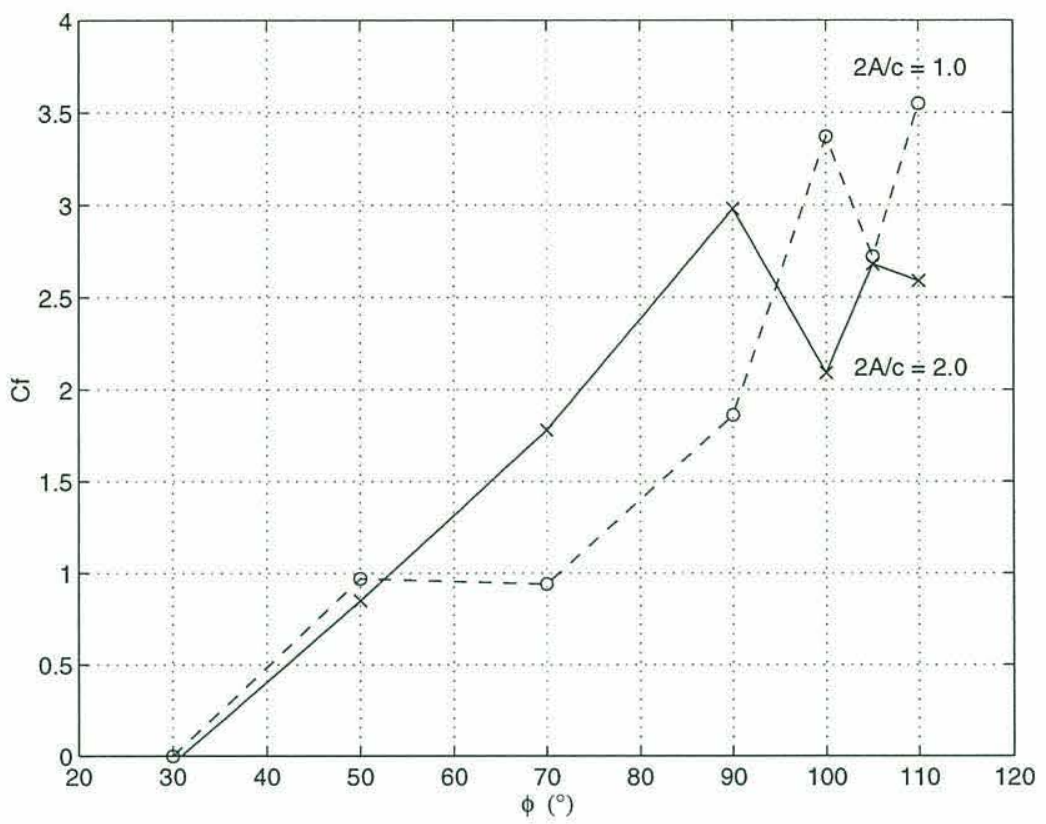

Figure 4-41: Thrust coefficient variation with phase angle for $\frac{2 A}{c}=1.0,2.0$. The kinematic data for these runs are listed in Table 4.1.

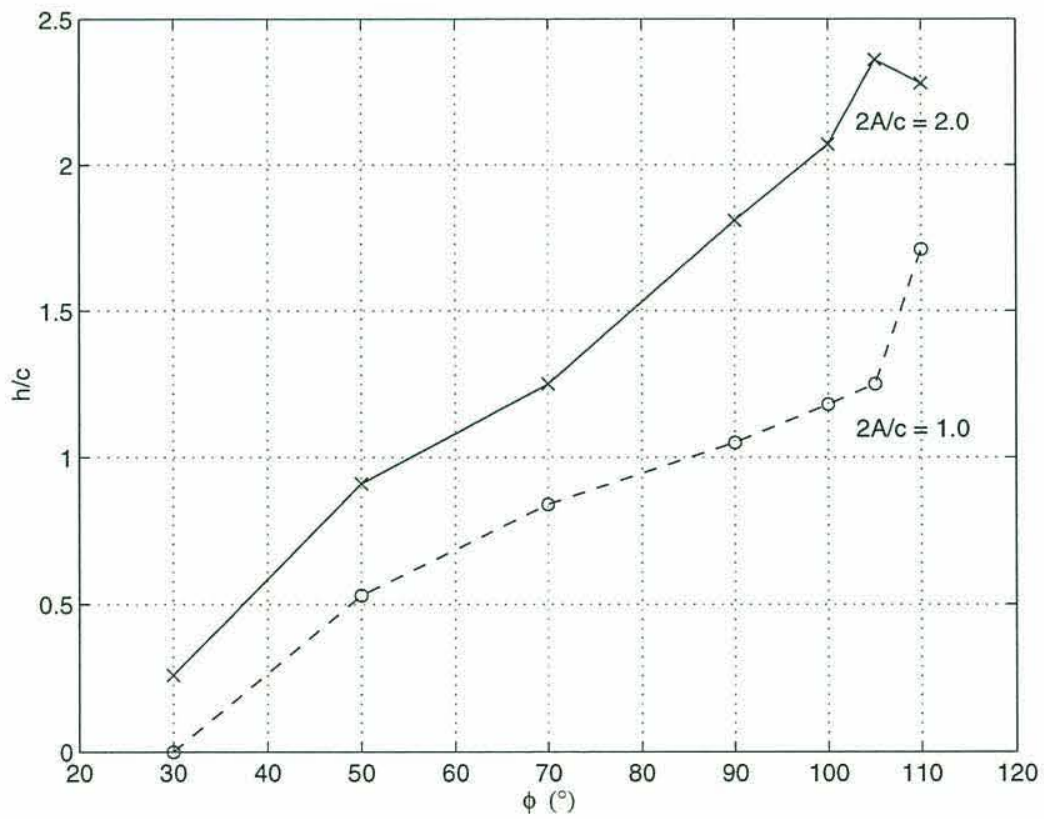

Figure 4-42: Wake width variation with phase angle for $\frac{2 A}{c}=1.0,2.0$. The kinematic data for these runs are listed in Table 4.1. 


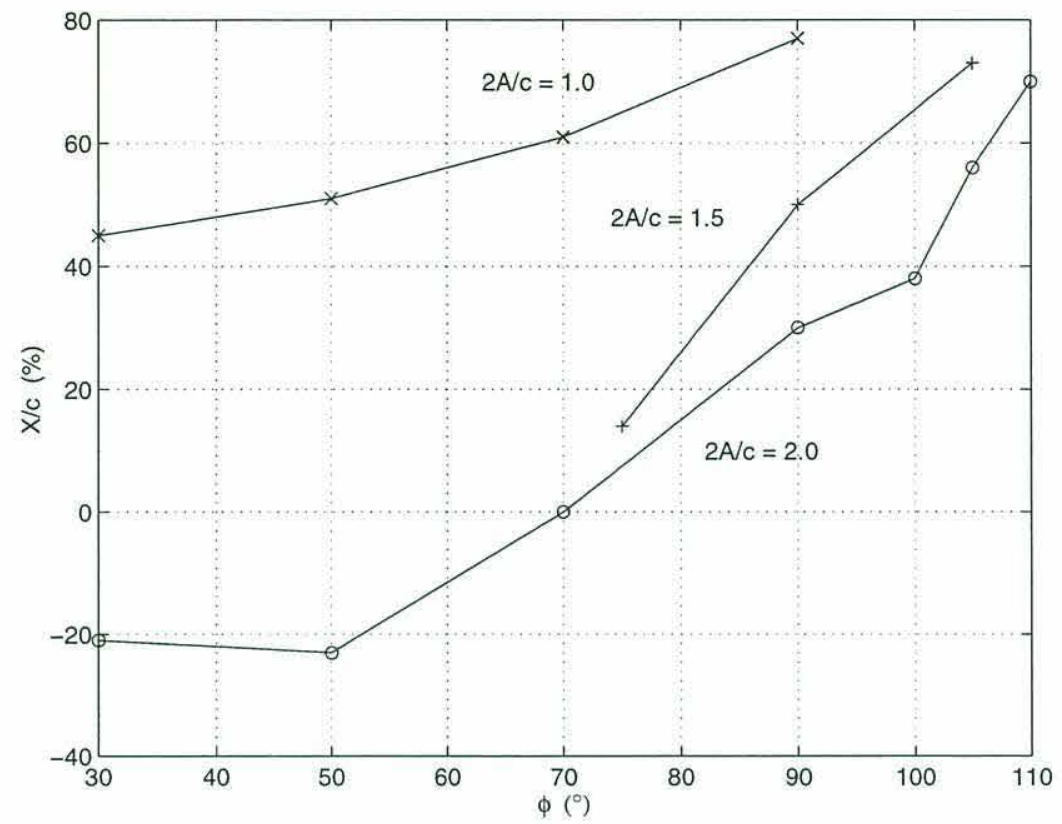

Figure 4-43: Stall vortex location along the foil at the position of maximum heave as a function of phase angle. $X / c$ is the chordwise position of the DSV along the foil with respect to the trailing edge. Negative numbers indicate a DSV that has left the foil surface.

increases both with Strouhal number and angle of attack. Even results outside of the M.I.T. parameter space qualitatively agree with the shape of the force coefficient curves.

Table 4.3 compares our data with data inferred from the M.I.T. data using bilinear interpolation to match Strouhal number and angle of attack. Contrary to the force measurements, for very low Strouhal numbers $(0.1-0.15)$ or low angle of attack $\left(\leq 5^{\circ}\right)$, we observed a drag wake where the force measurements indicate small thrust is generated. For the one case where our heave amplitude is the same as that for the M.I.T. data, $\frac{2 A}{c}=1.5$, our measurement compares very well, overpredicting the force measurement by only $9 \%$.

\subsubsection{Wake structure}

In the course of our experiments we have seen a variety of wake patterns which occur throughout the parameter space. Figure 4-45 summarizes the wake patterns as a function of trailing edge Strouhal number and angle of attack for large heave amplitude. We divide the $S t-\alpha_{\max }$ plane into regions of distinct wake patterns, with our observations labeled with point markers which follow the convention in Figure 4-9.

In region $\mathbf{A}$, the wake is a loosely organized Kármán street along the path of the trailing 


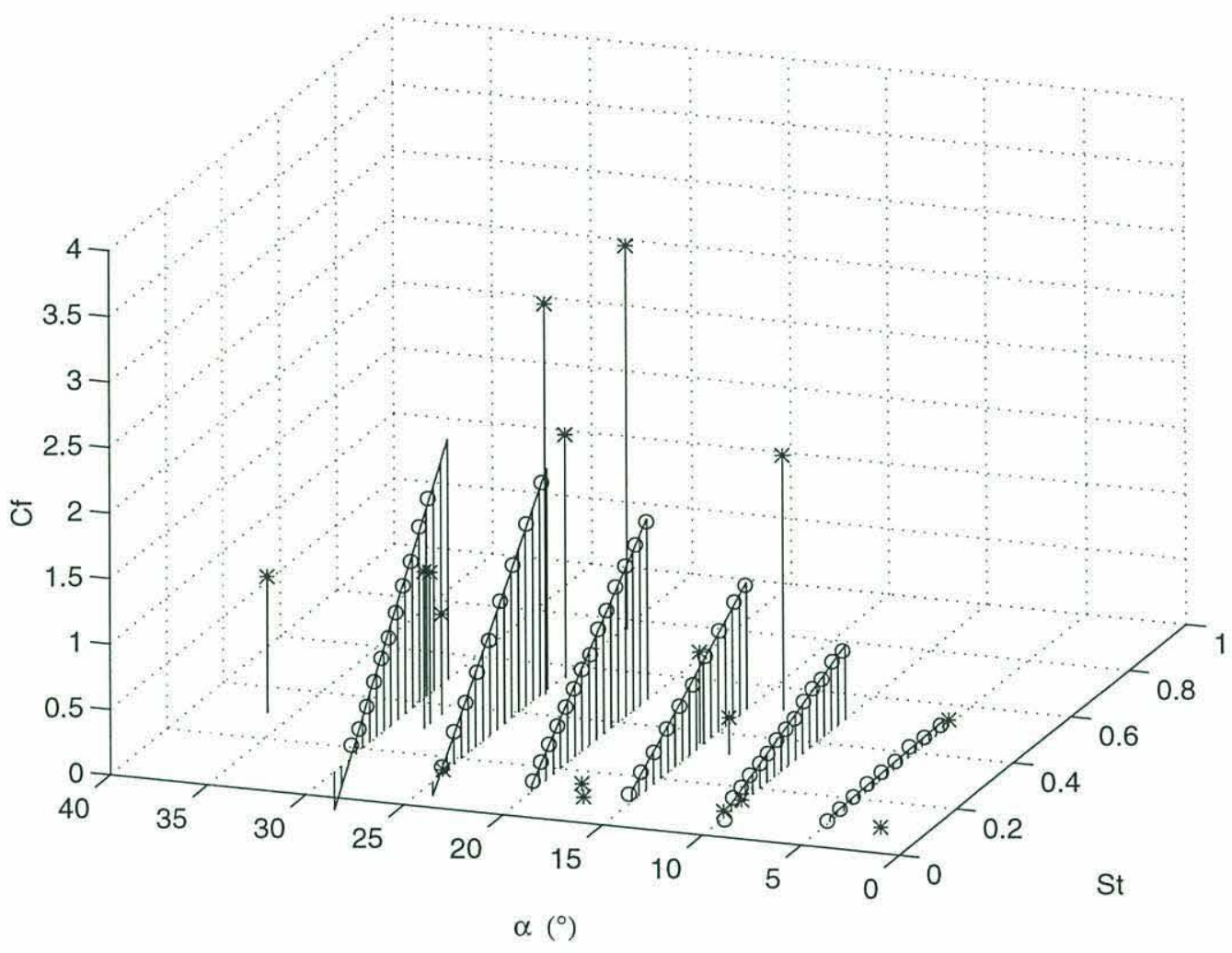

Figure 4-44: Experimental thrust coefficient as a function of maximum angle of attack and trailing edge Strouhal number. M.I.T. data at $\frac{2 A}{c}=1.5$ are marked with ' 0 ' marks and '*' marks indicate the new results using momentum analysis for $\frac{2 A}{c}=0.5,1.0,1.5$ and 2.0. 


\begin{tabular}{||l||c|c||c|c||}
\hline case & $\frac{2 A}{c}$ & $C_{f}$ & $\frac{2 A^{*}}{c}$ & $C_{f}^{*}$ \\
\hline \hline & & & & \\
fa04 & 2.0 & 0 & 1.5 & 0.06 \\
fa05 & 2.0 & 0 & 1.5 & 0.06 \\
fa06 & 2.0 & 0 & 1.5 & NIS \\
fa16 & 2.0 & 0 & 1.5 & 0.10 \\
fa17 & 2.0 & 0 & 1.5 & 0.21 \\
fa18 & 2.0 & 0 & 1.5 & 0.13 \\
fa01 & 2.0 & 1.04 & 1.5 & NIS \\
fa02 & 2.0 & 1.20 & 1.5 & 0.75 \\
fa03 & 2.0 & 0.28 & 1.5 & 0.48 \\
fa07 & 2.0 & 2.98 & 1.5 & 1.64 \\
fa08 & 2.0 & 1.95 & 1.5 & 0.85 \\
fa09 & 2.0 & 0 & 1.5 & NIS \\
fa10 & 0.5 & 0 & 1.5 & 0.06 \\
fa11 & 0.5 & 0.77 & 1.5 & 0.88 \\
fa12 & 0.5 & 2.93 & 1.5 & NIS \\
fa13 & 1.0 & 0 & 1.5 & 0.06 \\
fa14 & 1.0 & 1.16 & 1.5 & 0.96 \\
fa15 & 1.0 & 1.86 & 1.5 & 1.92 \\
fa36 & 1.5 & 0.70 & 1.5 & 0.64 \\
\hline
\end{tabular}

Table 4.3: Comparison of force coefficient results with M.I.T. data. $C_{f}$ is calculated with momentum analysis, $C_{f}^{*}$ is inferred by linear interpolation of direct force measurements at $\frac{2 A}{c}^{*}=1.5$. Most of our measurements were done at $\frac{2 A}{c}=2.0$, a different amplitude than the force measurements. NIS indicates that our experiment lies outside the force measurement parameter space. 
edge. In B, a Kármán street forms as in region A, but in addition we see a sluggishly organized leading edge vortex, even at low angles of attack. In $\mathbf{C}$, two leading edge vortices are shed per period forming a jet wake. Contrary to our expectations, distinct vortices do not form at the trailing edge. Rather, small vortices organize in the shear layer emanating from the trailing edge. In region $\mathbf{D}$, four vortices are shed per cycle, two originating from the leading edge and two from the trailing edge, which pair to form a mixed wake. In $\mathrm{E}$, four vortices per cycle form which all originate from the trailing edge. In region $\mathbf{F}$ where the angle of attack is extremely high $\left(\alpha_{\max }>50^{\circ}\right)$, piston type shedding from both edges produces a mixed wake.

From Figure $4-45$ it is readily surmised that for large amplitude motions $\left(\frac{2 A}{c}=2.0\right)$, distinct jet patterns form in a large region of the parameter space: $0.3<S t<0.5$ and $13^{\circ}<\alpha_{\max }<36^{\circ}$. For this heave amplitude, all of these jet patterns are comprised of vortices formed by leading edge separation.

At lower heave amplitudes, the wake pattern map of Figure 4-45 changes. For $\frac{2 A}{c}=1.0$, we begin to see slight leading edge separation in region $\mathbf{A}$. Region $\mathbf{C}$ still produces jets dominated by two leading edge vortices per cycle. However, trailing edge shedding begins to contribute to the jet wake by combining with the leading edge vortices. The merging process is synchronized such that in the near wake, same signed trailing and leading edge vortices immediately coalesce into one vortex. The upper Strouhal number bound of region C moves to 0.55 , because for this amplitude the shape of the angle of attack is still a flattened sinusoid, and not multi-peaked as in the $\frac{2 A}{c}=2.0$ case.

For very low heave amplitude $\left(\frac{2 A}{c}=0.5\right)$, the trailing edge dominates jet formation in region $\mathbf{C}$ rather than the leading edge as in the other cases. For this amplitude, region $\mathbf{C}$ extends out to $S t=0.76$. Again, this is due to the fact that the angle of attack profile remains a flattened sinusoid for this case.

The switch from leading edge to trailing edge generated vorticity when the heave amplitude is reduced is explained by looking at the rate of change of the angle of attack, $\Omega^{*}$. In the $\frac{2 A}{c}=0.5$ cases, the reduced frequency is high enough that the rotation of the foil dominates the dynamic stall process (Ohmi et al. $[82,83]$ ). Consider the case of a foil heaving upwards such that the pressure side of the foil faces the heave direction. Because the flow is dominated by the rotation and not the translating flow, pitching up of the foil will encourage development of a stall vortex and pitching down will restrain vortex development. When 


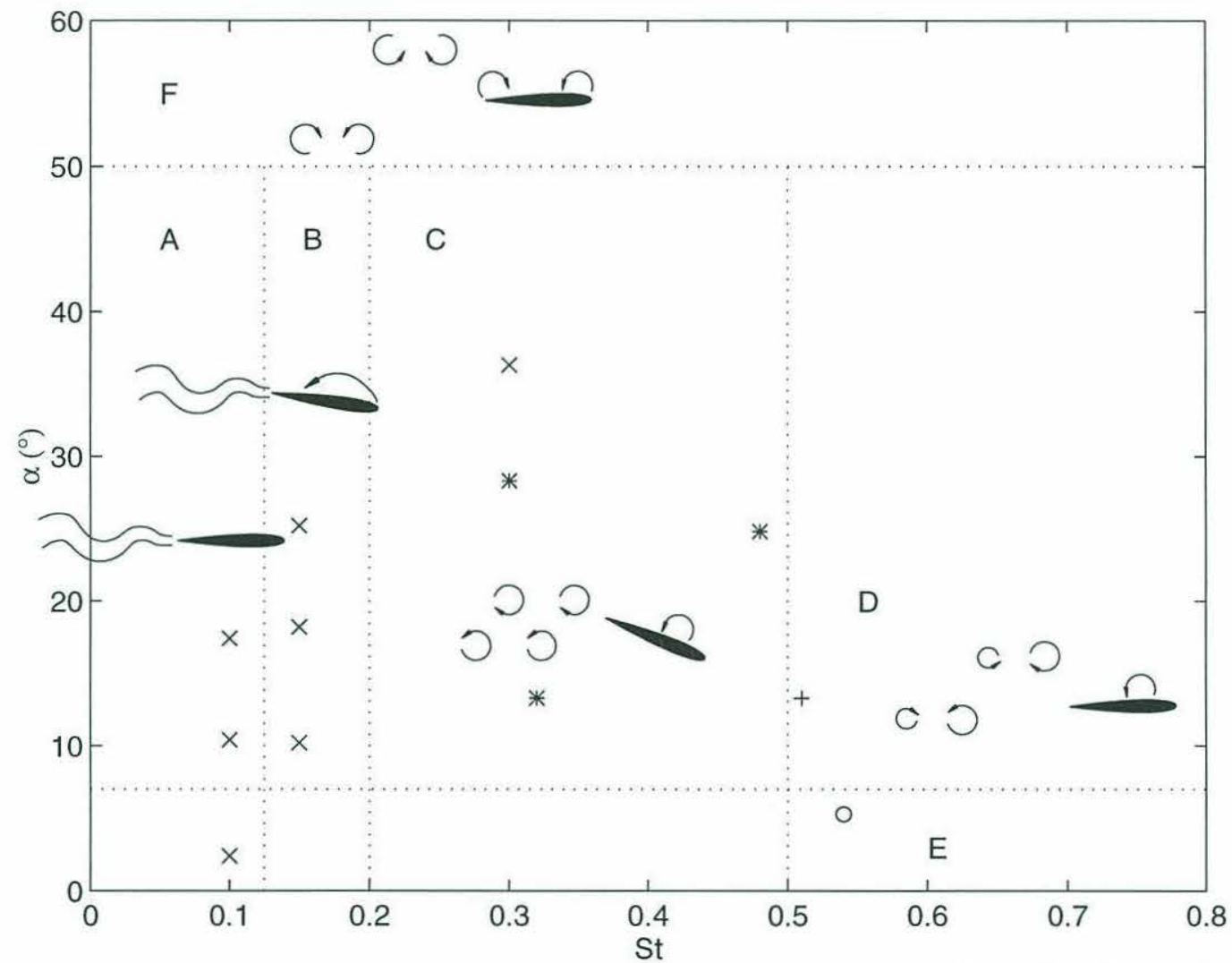

Figure 4-45: Wake patterns as a function of Strouhal number and angle of attack for $\frac{2 A}{c}=2.0$. The point markers mark the location of our experiments and follow the convention in Figure 4-9 with respect to the shape of the angle of attack profile. 
the phase angle is $90^{\circ}$, the foil pitch is declining to zero as the foil approaches the heave maximum, acting to restrain stall vortex growth. This scenario applies for all the heave amplitude cases. However, when the heave amplitude is very low, the rate of change of the angle of attack is much larger for a given Strouhal number. The pitch rate is sufficiently high to completely restrain leading edge vortex development. Visbal and Shang [125] noted the same behavior that increasing in the pitch rate towards the suction side of the foil delays the stall process.

\subsubsection{Multiple vortices per cycle}

In nearly all of our observations, the foil sheds two vortices per oscillation cycle, either from the trailing or leading edge. Multiple vortex shedding occurred only in cases fa08 and fa09, where the angle of attack profile was multi-peaked (Table 4.1) and the Strouhal number was moderately high $(S t \approx 0.5)$. In case fa08, the angle of attack profile is a flattened sinusoid with added peaks at the corners of the main peak, reminiscent of Gibbs' "ringing" phenomenon. The wake consists of four vortices per cycle, two from the leading edge and two from the trailing edge. The leading edge vortices form when $\Omega^{*}$ goes to zero at the main angle of attack peak (twice per cycle). The trailing edge vortices form when the angle of attack changes sign (twice per cycle). The vortices pair to form a mixed wake as indicated in the region $\mathbf{D}$ diagram in Figure 4-45.

Case $\mathrm{fa} 09$ is fundamentally different than case fa08 in that the angle of attack is low enough $\left(\alpha_{\max }=5^{\circ}\right)$ that leading edge separation does not occur. The angle of attack profile is triple-peaked which means that there are three cycles of $\alpha(t)$ per heave cycle. A small trailing edge vortex forms each time the angle of attack changes sign. These vortices pair to form a mixed wake which does not produce thrust.

Other authors have observed stable multiple vortex $(>2)$ shedding modes for small amplitude oscillations in pitch only. Koochesfahani $[57,58]$ observed four and six vortices per cycle for foils pitching only with small angles of attack $\left(\alpha_{\max }=4^{\circ}\right)$ and low Strouhal number (0.1). Bratt [17] also observed the four vortex cycle at low frequencies for a foil pitching less than $1^{\circ}$. In these cases, the angle of attack profile smoothly varies as a sinusoid and does not have additional angle of attack sign changes or rapid variations to initiate vortex shedding. Thus, it seems that in these cases, the multiple vortices are related to a shear layer instability. Although these cases are interesting, they are not significant for 
thrust production.

\subsection{Conclusions}

In this chapter, we began with a simple goal: to survey flapping foil wakes and ascertain the relationship between wake structure and thrust generation. We conducted an array of experiments, spanning the parameter space in four dominant kinematic variables: heave amplitude, wake Strouhal number, maximum angle of attack and phase angle between heave and pitch. For each experimental case, we identified the wake structure, the strength of wake vortices and the thrust coefficient. Our experiments uncovered a myriad of wake patterns which include leading edge separation and reattachment, trailing edge vortex generation and bluff body type drag wakes.

The propulsive performance of a flapping foil is influenced significantly by the kinematics, and hence so is the wake structure. We can make the following conclusions about flapping foil performance:

- For high efficiency and high thrust conditions, the reverse Kármán street wake pattern is ubiquitous.

- Leading edge separation significantly affects, and often dominates, the wake structure. Leading edge separation occurs for a wide range of Strouhal numbers and for angles of attack as low as $10^{\circ}$.

- Thrust and wake vortex strength increase with increasing Strouhal number and angle of attack.

- The shape of the angle of attack profile significantly influences wake structure. Sinusoid and square wave profiles produce the cleanest wakes with two vortices shed per cycle. Multiple peaks in the angle of attack profile cause additional shedding which mixes the wake.

- The phase angle influences the development of dynamic stall vortices which affects overall thrust generation. Favorable placement of the dynamic stall vortices during the oscillation cycle is possible at phase angles below $90^{\circ}$. 
- The rate of change of the angle of attack affects the formation of leading edge vortices. At low amplitudes, the rate of change of angle of attack is high enough to completely restrain dynamic stall.

Our experiments have revealed the complexity of wake structure that was previously unexpected. Future work in this area looks promising, especially in the manipulation of the stall process for maximum efficiency. Although our experiments were conducted at a moderately low Reynolds number, the stall process appears not to vary significantly with Reynolds number. The location and time of initial boundary layer breakup are asymptotically independent of Reynolds number [29]. Increased Reynolds number would certainly affect shedding at the trailing edge due to thickening of the boundary layer in that region. We hope to be able to do experiments in the future with simultaneous DPIV and force/efficiency measurements at Reynolds numbers more appropriate to engineering applications. 


\section{Chapter 5}

\section{Vorticity control for enhanced propulsive efficiency}

\subsection{Introduction}

In this chapter, we address the concept of vortex control for the purpose of efficiency enhancement and drag reduction. We begin by presenting experimental results for a new kind of vortex generator. A D-section cylinder heaving in the transverse direction generates an array of vortices resembling a Kármán vortex street in form. An oscillating foil placed inside this vortex street can manipulate the cylinder vortices through several mechanisms. Cylinder vortices are moved due to suction forces from the foil motion; they can be reinforced, annihilated or paired with foil vortices to produce a variety of wake patterns. In this chapter we present DPIV results for an array of experiments using a flapping foil to control upstream vorticity in the form of a drag wake.

\subsection{Background}

Our motivation for this chapter stems primarily from two recent works at the Massachusetts Institute of Technology. In 1993, Gopalkrishnan [38, 39] studied the interaction of a low angle of attack flapping foil with an oncoming vortex street generated by a D-cylinder. Visualizations were done at cylinder Reynolds number $R_{d}=540$ which revealed distinct interaction modes between the cylinder vortex street and vorticity generated by the flapping foil. Force measurements at $R_{d} \approx 2 \times 10^{5}$ confirmed that the efficiency of the tandem arrangement depends on interaction kinematics. 
Gopalkrishnan used Kalliroscopic fluid (see Chapter 3 for description) in a shallow tank with the same towing carriage and oscillation apparatus used in our own experiments. A systematic search of the parameter space showed that the spacing between the cylinder and foil, that is, the phasing of the foil encounter with respect to the cylinder vortex street is the dominant parameter.

Three distinct wake interaction modes were identified and are shown schematically in Figure 5-1. One arrangement generates a wake in which the cylinder vortices pair with oppositely signed foil vortices. The pairs' mutual induction moves them away from the centerline in "mushroom" patterns, broadening the wake. A second interesting mode occurs when the cylinder vortices join with oppositely signed foil vortices and destructively merge. The resulting weak vortices remain near the wake centerline, indicating that neither thrust nor drag are generated. The third distinct mode is the opposite case: cylinder vortices join same signed foil vortices in a constructive arrangement to produce an energetic vortex array along the centerline.

Gopalkrishnan's force and efficiency measurements indicate that the destructive arrangement corresponds to a peak in the system efficiency. Likewise, the constructive and pairing modes have low efficiency. The destructive case presents us with an interesting concept in flow (vortex) control. In the presence of upstream vorticity from a drag producing body, an oscillating foil in the wake can recover the drag vortices, reducing the overall drag and perhaps producing efficient thrust in the process. This concept is the motivation for our experimental survey of vortex control using a flapping foil.

Streitlien [112, 113], also motivated by the intriguing results of Gopalkrishnan [38, 39], simulated a flapping foil in the presence of upstream vorticity. Using a potential flow based code, Streitlien generated upstream unsteadiness by introducing bundles of point vortices in a simulated Kármán vortex street. Both destructive and constructive merging modes were observed depending on the encounter phase of the foil with respect to the upstream vortices. Destructive merging occurs when the foil intercepts the upstream vortices head on, and constructive merging when the foil slaloms to avoid the upstream vortices. The highest efficiency occurs for the destructive mode in which the foil is heavily loaded, yet produces the most reduced wake signature.

The interaction of a foil with impinging vorticity is a classic problem in aerodynamics in the blade-vortex-interaction (BVI) problem. In rotorcraft, the blades operate in each 
(a)

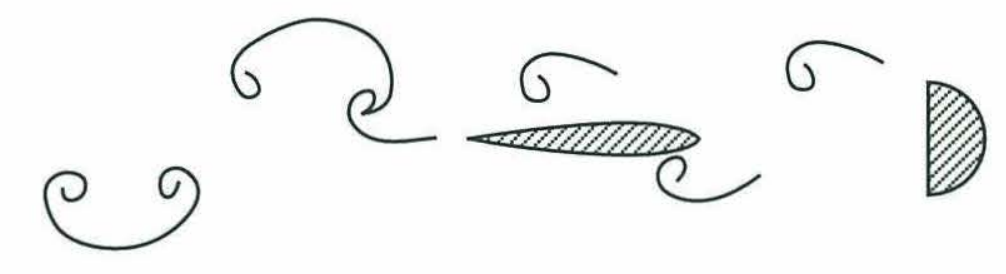

(b)

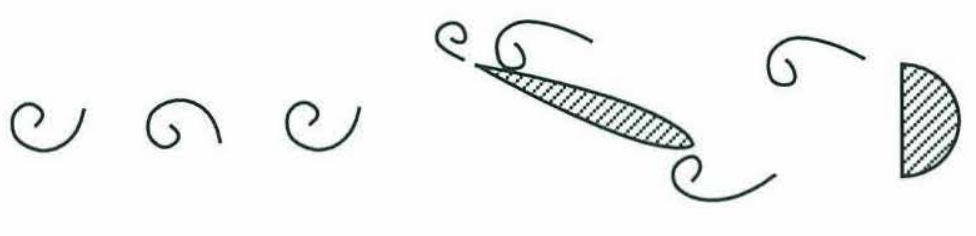

(c)
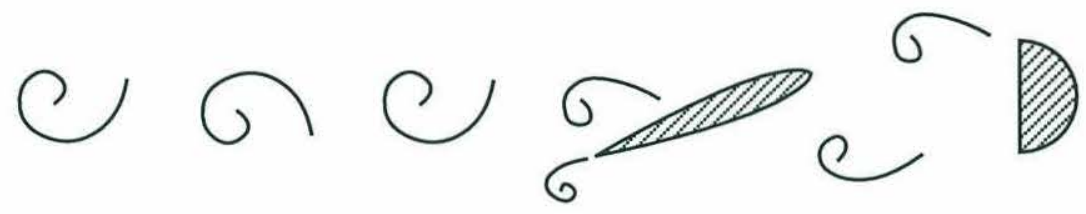

Figure 5-1: Wake interaction modes observed by Gopalkrishnan $(1993,1994)$ for tandem D-cylinder and foil. (a) vortex pairing: cylinder and foil vortices of opposite signs combine to form vortex pairs. (b) destructive merging: oppositely signed vortices combine and annihilate each other and diminish the wake. (c) constructive merging: same signed vortices merge to form an energetic wake.

others wakes: the tip vortex from the previous blade is ingested by the rotor disk and comes in close proximity to the next blade. This loads the blade generating an impulsive noise. Acoustic research has shown that the most intensive noise pulse occurs when the vortex is parallel to the blade span. Thus, several researchers have concentrated on this 2-D BVI problem.

Experiments in passive (stationary blade) BVI problems have revealed several possible interactions. Świrydczuk [114] observed "interactional" vortices develop in the wake of the blade in the opposite orientation of the incident vortex, causing the incident vortex to disintegrate. Booth et al. $[14,12,13]$ observed variation in incident vortex trajectory, size and shape, depending on the interaction kinematics. Vortex bursting and splitting were observed, with part of the original vortex continuing on weakly while other pieces disintegrate. Robinson et al. [93] studied the problem of tandem airfoils noting a myriad of "tandem vortex complexes" incorporating leading edge and trailing edge vortices. In summary, the research in BVI has demonstrated that a variety of wake interactions can occur, dramatically changing the wake structure and loading on the foil. 
An interesting application of active wake control is presented by Koochesfahani and Dimotakis [59]. They demonstrate that it is possible to cancel the effects of an externally generated disturbance in a fully developed shear layer using a pitching foil and feedback control. Proper selection of phase and amplitude of a pitching control foil located downstream of a forcing foil can effectively cancel the disturbances introduced by the forcing foil.

\subsection{The D-cylinder as a vortex generator}

In this section we investigate the wake behind an oscillating half cylinder or "D-cylinder" as a vortex generator. We begin by quantifying the wake structure as a function of the cylinder kinematics. A D-cylinder oriented with the flat face pointed downstream has fixed separation points at the corners. Unlike whole cylinder shedding, the sharp corners severe the separating shear layer into discrete patches of vorticity. When oscillated, the vortex shedding synchronizes to the motion of the cylinder and the resultant wake is a well organized staggered array of oppositely signed vortices.

Several authors have studied vortex shedding from oscillating circular cylinders $[8,103$, 111, 138]. Lateral oscillations can synchronize the natural vortex shedding to the oscillation frequency at frequencies roughly $30 \%$ below and $20 \%$ above the natural shedding frequency according to Honji and Taneda [48]. The wake structure depends heavily on the frequency and amplitude of the oscillation. Zdravkovich [148] concluded that the shed vortex forms when the cylinder is near the maximum amplitude on the opposite side to the vortex when the frequency is in the lower synchronization range and on the same side for the higher synchronization range.

Few researchers have studied D-section cylinders because as a bluff body they are rather uninteresting. Simmons [110] studied the turbulent wake of a wide D-shaped cylinder comprised of a half cylinder and and square rear section. As in our experiments, Simmons was interested in strong shedding to verify his hot-wire technique to measure the convection speed of wake vortices. 


\subsubsection{Definitions}

We consider sinusoidal heave of the D-cylinder with amplitude $A$ and frequency $f$ referenced to the center of the cylinder as if it were whole

$$
y(t)=A \sin (2 \pi f t)
$$

The natural shedding frequency of a stationary cylinder, $f_{s}$, is given by the Strouhal number based on the cylinder diameter

$$
S t_{d}=\frac{d f_{s}}{U}
$$

where $U$ is the tow speed. For a circular cylinder, the Strouhal number is nearly constant at 0.2 for Reynolds numbers from 200 to $10^{5}$ [11]. For a stationary D-cylinder, the Strouhal number for natural shedding was measured by Gopalkrishnan [39] to be approximately 0.19 for Reynolds numbers from 300 to 650 . We expect the best vortex streets to occur when the cylinder oscillation frequency is near the natural shedding frequency. We also define an oscillation Strouhal number based on the amplitude of heave

$$
S t_{A}=\frac{2 A f}{U}
$$

Experiments were done in the DPIV and visualization tank (Figure 4-7) with the oscillation apparatus described in Chapter 4. As in previous studies, the laser sheet was oriented in the horizontal plane at the tank mid-depth. DPIV measurements of the velocity field were made over a range of amplitudes and frequencies. The $1.91 \mathrm{~cm}$ diameter D-cylinder was machined out of aluminum and anodized to a flat black finish. The corners of the flat face were left unchamfered to give relatively sharp shedding points. No special attention was given to the cylinder surface except that it was free of macroscopic defects. The cylinder length is large (37.3 diameters) to guarantee strong shedding correlation over most of the length. One end of the cylinder was free near the tank bottom and the other pierced the free surface and attached to the oscillation mechanism. No endplate was used on the free end.

The double amplitude was varied between zero and four diameters at frequencies from

zero to $0.6 \mathrm{~Hz}$. The tow speed was fixed at $U=2.82 \frac{\mathrm{cm}}{\mathrm{s}}(\approx 1.5$ diameters per second $)$ 
or Reynolds number $R_{d}=540$. The cylinder was started from rest in a still tank at least 50 diameters away from the DPIV viewing area. Hence, the wake is expected to be fully developed in the viewing area. As in previous chapters, all data are presented in the inertial reference frame with near-body and shadowed regions removed.

Figure 5-2 is a schematic diagram of the D-cylinder and its wake. We expect the wake structure to depend on the amplitude and frequency of oscillation, the tow speed and the cylinder diameter. The wake parameters of interest are the streamwise spacing between same-side vortices $s$, the wake width $h$, the circulation of the vortices $\Gamma$, and the velocity defect behind the cylinder $\bar{u}$.

Table 5.1 summarizes the parameter space and results of the oscillation experiments. The last column indicates whether or not the vortex array remains rectangular or tends to spread laterally downstream. The streamwise spacing of vortices is normalized with respect to the spatial wavelength of the motion, $\lambda=\frac{U}{f}$. The average circulation of the wake vortices $\bar{\Gamma}$ is computed by averaging the circulation of each of the four most recently shed vortices. The circulations of individual vortices are integrated along circular contours using equation (2.5). The radius of the integration contour is that radius where the maximum circulation occurs, or $2 \mathrm{~cm}(\approx d)$, whichever is smaller.

The average wake defect velocity $\bar{u}$ is computed by averaging the horizontal velocity component at twenty spatial locations over one wavelength, starting one diameter downstream of the cylinder. Temporal variations in the wake defect are neglected. The drag coefficient $C_{D}$ is computed using the same momentum flux integral approach used in Chapter 4 . The 2-D drag coefficient is defined in the usual way for unit span

$$
C_{D}=\frac{D}{\frac{1}{2} \rho U^{2} d}
$$

Figures 5-3 and 5-4 show the velocity field and vorticity contours for case cyl03 where the cylinder heaves with double amplitude $\frac{2 A}{d}=1.0$ and cylinder Strouhal number $S t_{d}=0.27$. In the figures, the cylinder has traveled from left to right and is shown in its actual position near the centerline of motion moving upwards. This motion produces a strong staggered array of discrete vortices.

Figure 5-5 summarizes the performance of the oscillations as a function of heave ampli- 


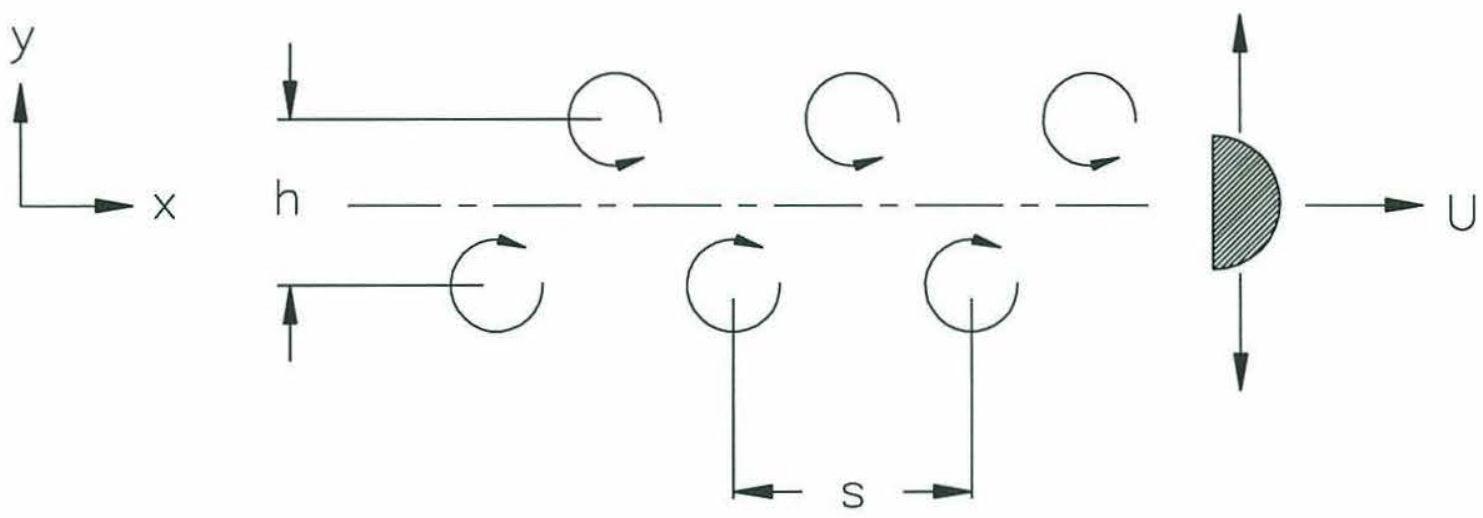

Figure 5-2: Schematic diagram of D-cylinder motion and wake structure

\begin{tabular}{||c||c|c|c|c||c|c|c|c|c|c|c||}
\hline case & $\frac{2 A}{d}$ & $\begin{array}{c}f \\
(\mathrm{~Hz})\end{array}$ & $\begin{array}{c}S t_{A} \\
\frac{2 A f}{U}\end{array}$ & $\begin{array}{c}S t_{d} \\
\frac{d f}{U}\end{array}$ & $\frac{s}{\lambda}$ & $\frac{h}{d}$ & $\left|\frac{\bar{u}}{U}\right|_{\max }$ & $\frac{\Gamma}{U d}$ & $C_{D}$ & $\begin{array}{c}\text { vortex } \\
\text { street }\end{array}$ & divergent \\
\hline cyl01 & 0 & 0 & 0 & 0 & - & - & - & - & - & poor & - \\
\hline cyl02 & 1.0 & 0.2 & 0.14 & 0.14 & - & - & 0.20 & - & 1.5 & poor & - \\
cyl03 & 1.0 & 0.4 & 0.27 & 0.27 & 0.59 & 2.0 & 0.98 & 2.3 & 3.0 & excellent & no \\
cyl14 & 1.0 & 0.5 & 0.34 & 0.34 & 0.56 & 2.1 & 0.90 & 2.2 & 0.6 & excellent & no \\
cyl04 & 1.0 & 0.6 & 0.41 & 0.41 & 0.70 & 2.4 & 0.94 & 2.0 & 1.6 & excellent & no \\
\hline cyl05 & 2.0 & 0.2 & 0.27 & 0.14 & - & - & 0.32 & - & 2.7 & poor & - \\
cyl06 & 2.0 & 0.4 & 0.54 & 0.27 & 0.67 & 3.6 & 0.90 & 2.7 & 5.4 & excellent & slight \\
cyl15 & 2.0 & 0.5 & 0.68 & 0.34 & 0.73 & 3.8 & 0.70 & 2.1 & 4.6 & excellent & no \\
cyl07 & 2.0 & 0.6 & 0.81 & 0.41 & 0.84 & 3.6 & 0.89 & 2.0 & 4.5 & excellent & no \\
\hline cyl08 & 3.0 & 0.2 & 0.41 & 0.14 & - & - & 0.31 & - & 2.7 & poor & - \\
cyl09 & 3.0 & 0.4 & 0.81 & 0.27 & 0.92 & 5.1 & 0.96 & 2.9 & 6.0 & good & yes \\
cyl16 & 3.0 & 0.5 & 1.02 & 0.34 & 0.89 & 6.1 & 1.11 & 1.8 & 7.1 & good & no \\
cyl10 & 3.0 & 0.6 & 1.22 & 0.41 & 1.02 & 5.1 & 0.90 & 2.0 & 6.7 & good & yes \\
\hline cyl11 & 4.0 & 0.2 & 0.54 & 0.14 & - & - & 0.17 & - & 0.5 & poor & - \\
cyl12 & 4.0 & 0.4 & 1.08 & 0.27 & 0.79 & 6.0 & 1.14 & 2.5 & 8.4 & mediocre & yes \\
cyl13 & 4.0 & 0.6 & 1.63 & 0.41 & - & - & - & - & - & poor & - \\
\hline
\end{tabular}

Table 5.1: Summary of D-cylinder experiments and results for $d=1.91 \mathrm{~cm}$ and $R_{d}=540$. 
tude and the heave velocity to tow speed ratio given as

$$
\frac{|\dot{y}(t)|}{U}=\frac{2 \pi f A}{U}=\pi S t_{A}
$$

Figure 5-5 clearly shows a region in the parameter space where the vortex generator performs well. If the frequency is too low, the wake fails to organize. The fluid immediately behind the cylinder follows the cylinder without forming distinct vortices. However, when the frequency is increased sufficiently, an organized wake forms which persists for 3 to 4 wavelengths downstream.

Figure 5-5 also shows that successful vortex generation depends heavily on the heave amplitude. At large heave amplitudes, $\frac{2 A}{d}>3$, the D-cylinder fails as a vortex generator due to vortex shedding in the transverse direction. These vortices disrupt the streamwise wake structure. Interestingly, the best amplitude and frequency combination occurs along

the line $\frac{2 A}{d}=\pi S t_{A}$ up to $\frac{2 A}{d} \approx 3$. In another form, this requires that $\pi S t_{d} \approx 1$ for good vortex generation.

Table 5.1 also shows that the wake parameters are highly variable. First, the streamwise spacing between vortices is not equal to the wavelength of the motion; the vortices convect with the cylinder. As the amplitude of heave increases up to $\frac{2 A}{d}=3.0$, the spacing between vortices approaches one wavelength. Interestingly, the wake defect velocity varies between $0.76 U$ and $1.04 U$ with no apparent correlation to frequency or amplitude. This may be due to the lack of a good statistical sample.

The wake width $h$ depends linearly on the heave amplitude with no apparent correlation to the frequency of oscillation. The strengths of the vortices $\bar{\Gamma}$ are roughly the same for all runs. Small variations between runs do not correlate to frequency or amplitude and are likely due again to the small statistical sample. One explanation is that the vortex strength is likely governed by the Reynolds number and not the transverse motion.

Figure 5-6 shows the variation of the drag coefficient as a function of cylinder Strouhal number $S t_{d}=\frac{f d}{U}$ and double amplitude of oscillation. Here, $f$ is the frequency of the motion rather than the natural shedding frequency of the cylinder. In all of our experiments, the shedding frequency is locked onto the oscillation frequency. The drag coefficient of a steady non-oscillating D-cylinder is given by Hoerner [47] to be 1.16 at $R_{d}=2000$. This is the value indicated in Figure 5-6 at zero amplitude. 


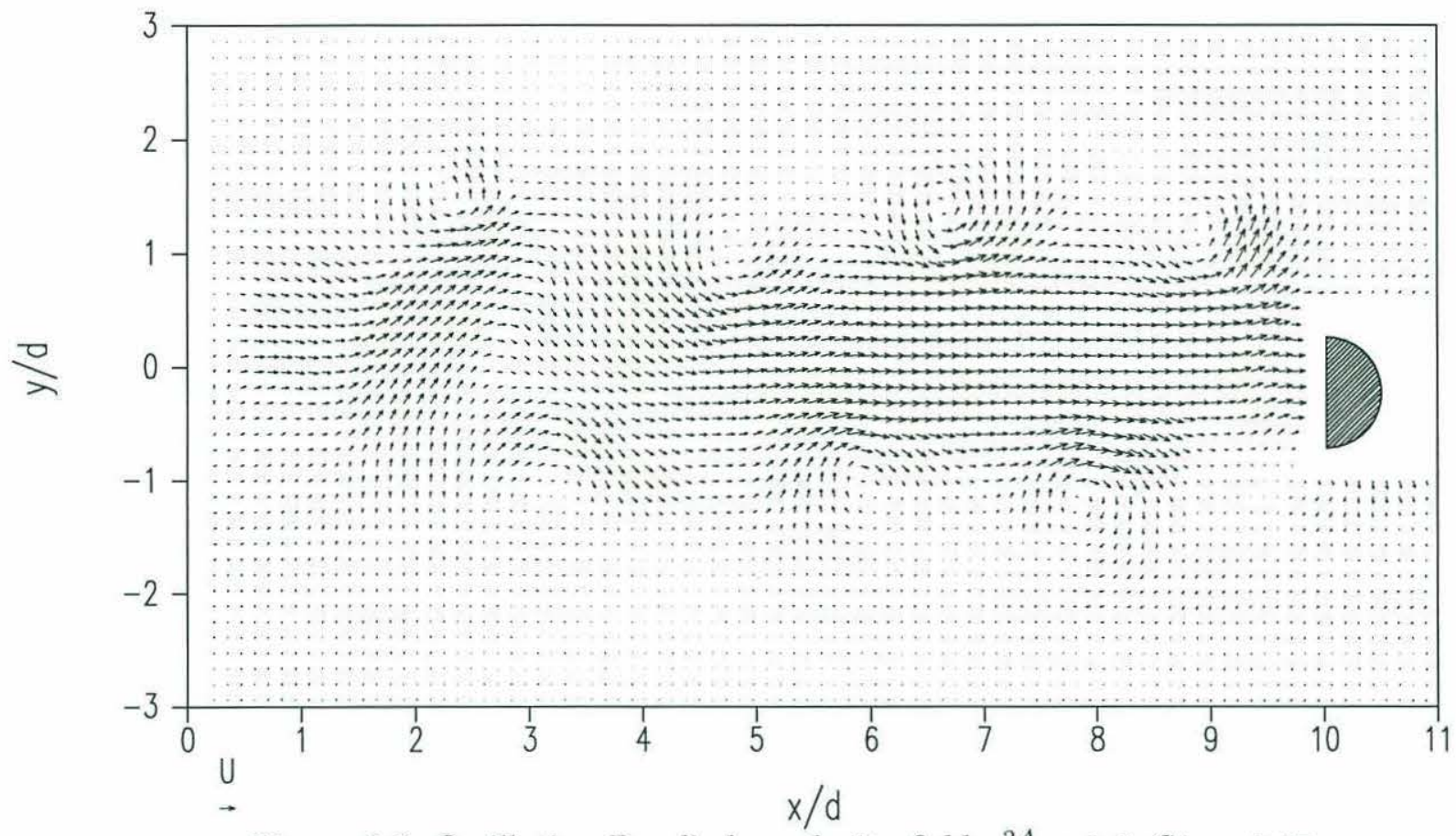

Figure 5-3: Oscillating D-cylinder velocity field. $\frac{2 A}{d}=1.0, S t_{d}=0.27$.

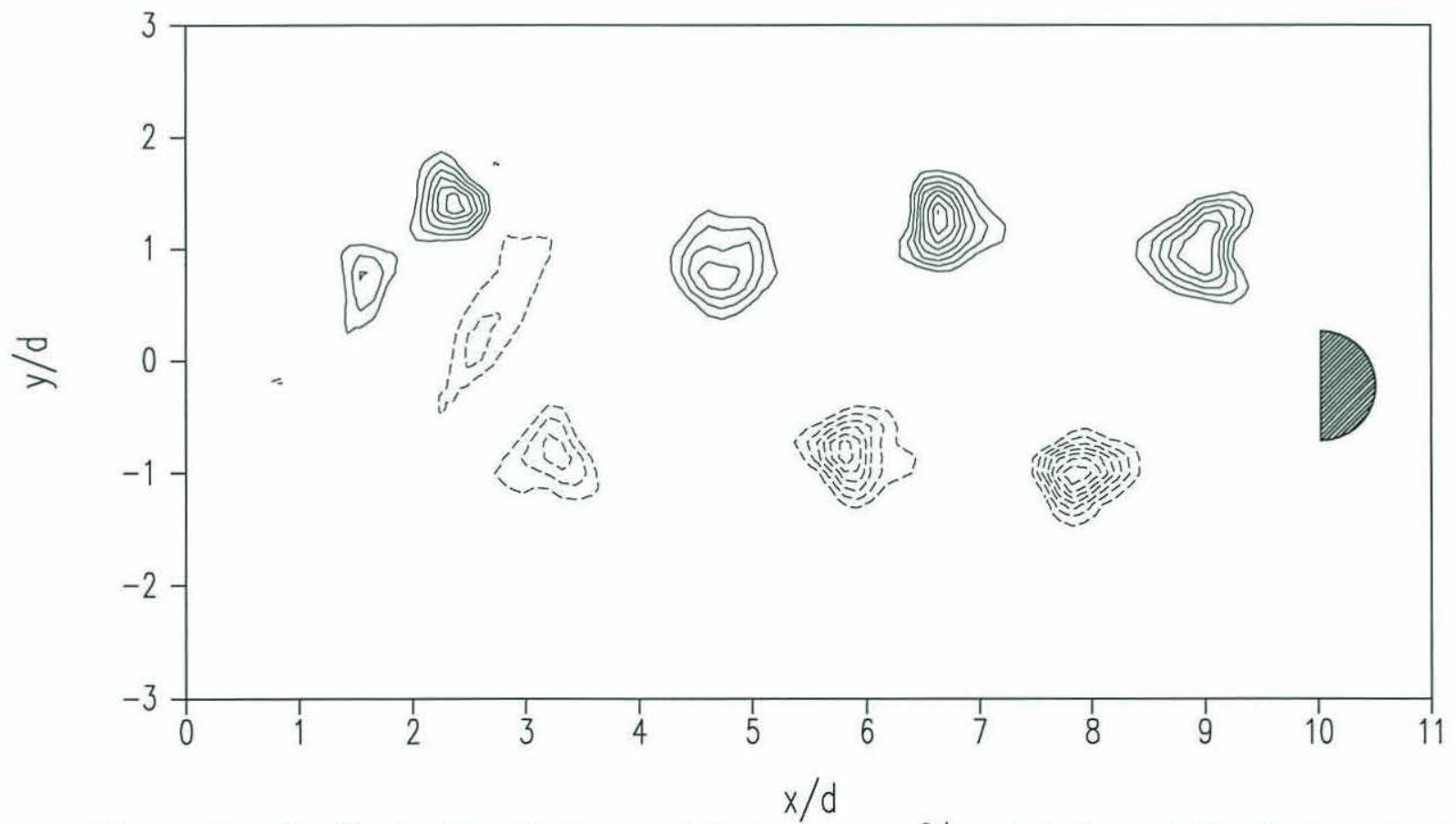

Figure 5-4: Oscillating D-cylinder vorticity contours. $\frac{2 A}{d}=1.0, S t_{d}=0.27$. Contour levels are from -10.0 to $10.0 \frac{1}{s}$ in $1.0 \frac{1}{s}$ increments excluding the zero contour. 


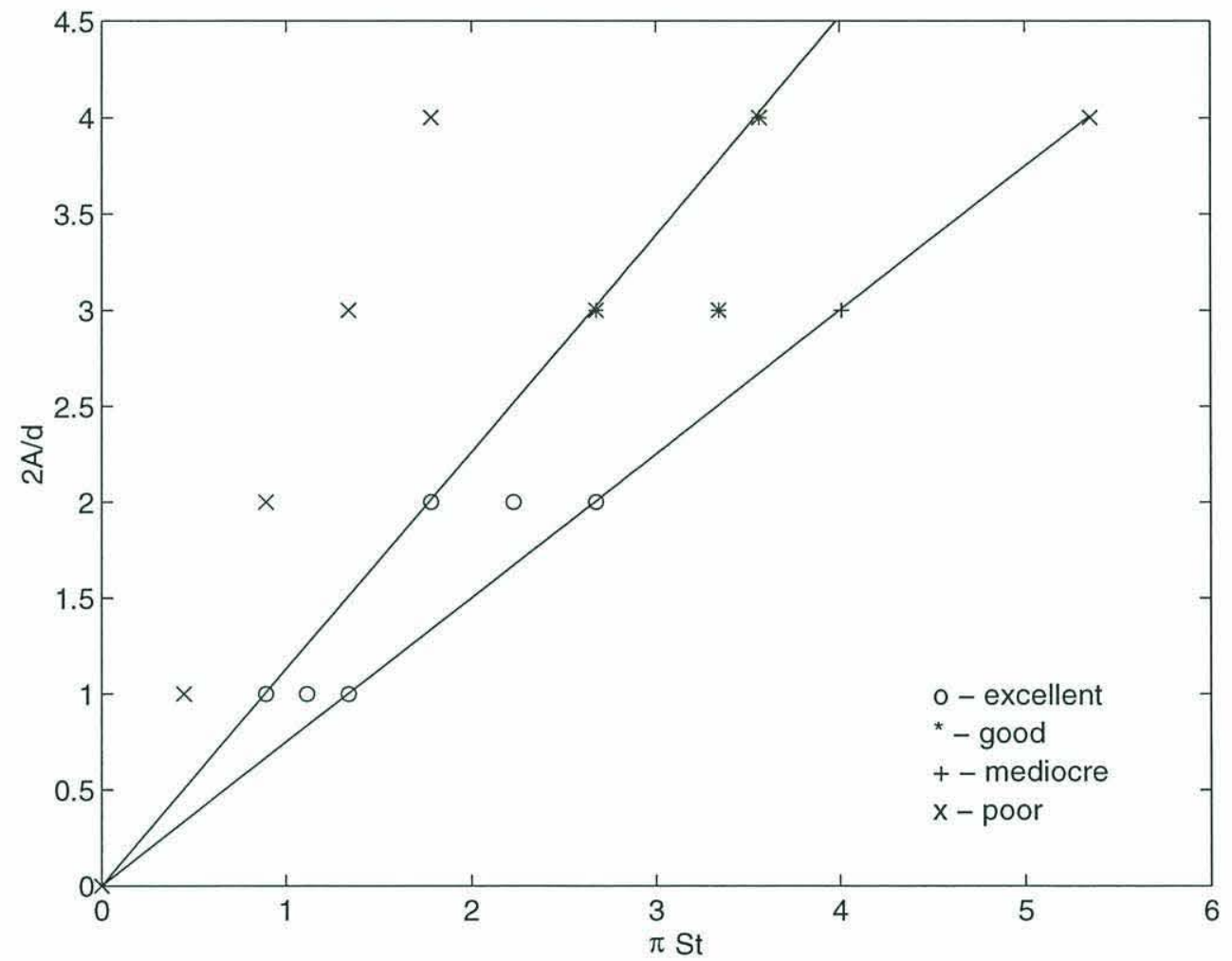

Figure 5-5: Performance of oscillating D-cylinder as a vortex generator as a function of heave amplitude and $\pi S t_{A}$, the ratio of heave velocity to tow speed. The plotted lines bound the region of good performance. 
As one would expect, the drag increases with increasing amplitude because the wake widens. The drag coefficient exceeds the steady drag by several factors even at moderate heave amplitudes. Interestingly, the drag decreases from the steady value for low heave amplitude and Strouhal number equal to 0.34 . This particular case was verified directly against a force measurement made at the Massachusetts Institute of Technology Ocean Engineering Testing Tank. My colleague Scott Miller was helpful in performing an experiment corresponding to the case cyl14 in Table 5.1 at $R_{d}=5700$ using the virtual cable testing apparatus (VCTA) in the large towing tank.

A wooden D-cylinder section with $2.54 \mathrm{~cm}$ diameter and $0.6 \mathrm{~m}$ span was suspended horizontally underwater via two faired surface-piercing struts attached to an oscillation apparatus and towing carriage. The cylinder section was equipped with large circular endplates and a precision piezoelectric force sensor. The entire VCTA structure was sinusoidally oscillated in heave with amplitude $\frac{2 A}{d}=1.0$ and Strouhal number equal to 0.34 , while translating at $0.224 \frac{\mathrm{m}}{\mathrm{s}}$. Details of the VCTA and the M.I.T. Ocean Engineering Testing Tank are provided in Miller [74].

The drag coefficient obtained by momentum flux considerations compares very well with the actual force measurement. Our momentum method predicts a drag coefficient within the standard deviation of the experimental data. This verifies that momentum flux dominates equation (4.5), rather than the time rate of change of momentum within the control volume or the unsteady pressure along the control volume boundaries.

In conclusion, the D-cylinder is an excellent vortex generator over a range of heave amplitudes and frequencies. The best vortex generation occurs for double heave amplitude between one and three diameters and cylinder Strouhal numbers near 0.3. Oscillation of the cylinder organizes the shed vorticity into a well ordered array of counter-rotating discrete vortices.

\subsection{Cylinder-foil tandem arrangements}

Our strategy for studying tandem cylinder-foil arrangements is based on our previous studies of flapping foils (Chapter 4) and the D-cylinder as a vortex generator. Previous work by Gopalkrishnan $[38,39]$ and Streitlien $[112,113]$ indicate that the dominant parameter is the spatial phase relationship between the upstream vortices and the foil motion. Hence, we 


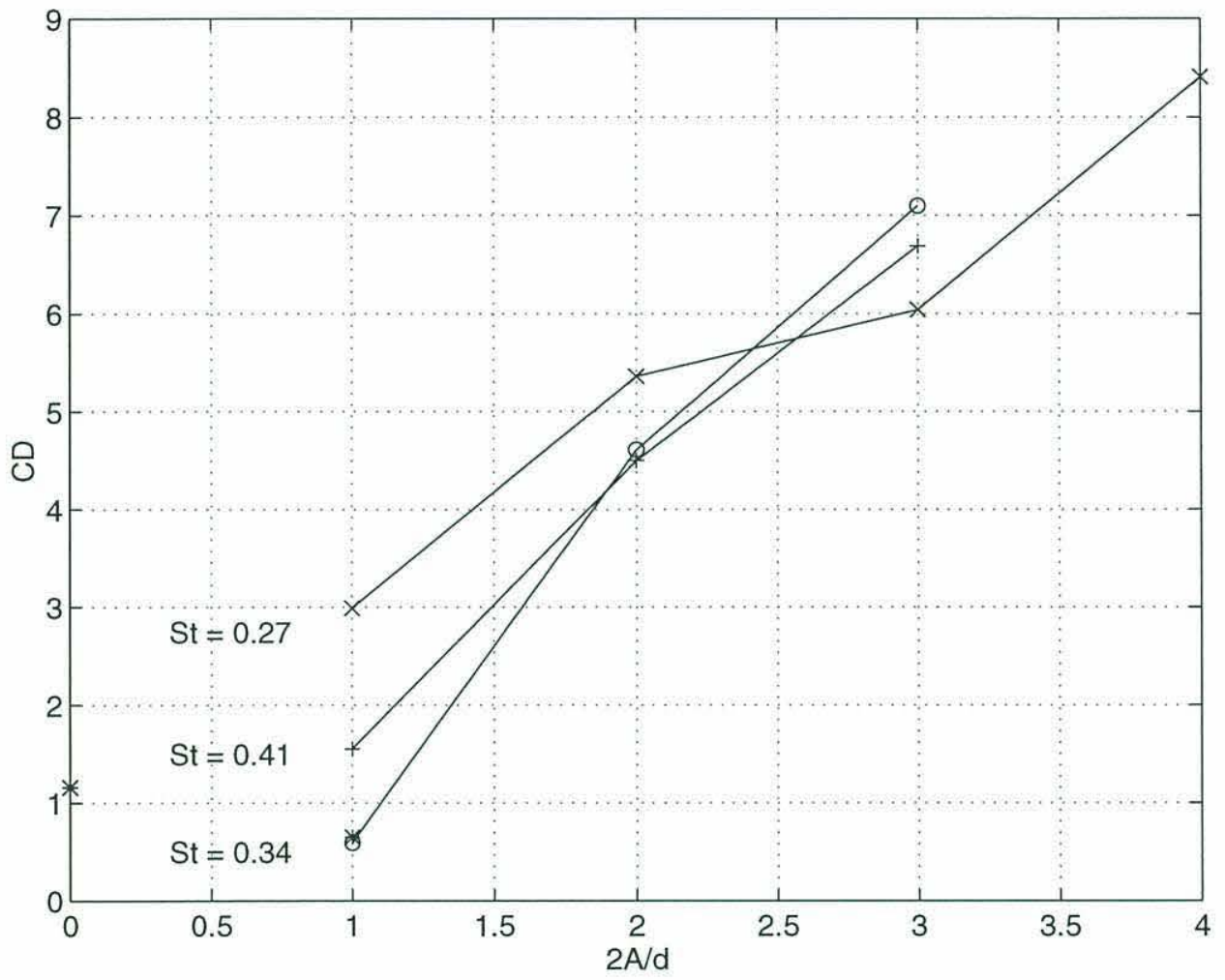

Figure 5-6: Drag coefficient of an oscillating D-cylinder as a function of Strouhal number and amplitude. The ' $*$ ' values indicate force measurements from Hoerner (1965) with no oscillation and our own measurement at $S t_{d}=0.34$ and $\frac{2 A}{d}=1.0$. 
began our survey by fixing the cylinder and foil motion, and varying the vortex encounter phasing by adjusting the spacing between the cylinder and foil. The cylinder and foil models are the same as those used in previous experiments already described. The foil chord to cylinder diameter ratio was fixed at $\frac{c}{d}=2.0$ which worked well in previous studies by Gopalkrishnan [39]. The magnitude of the vorticity generated by the cylinder and foil are similar in strength and should produce interesting interactions.

The cylinder and foil were mounted in tandem as shown in Figure 5-1 on the same oscillation apparatus used in previous experiments. The apparatus requires that the foil and cylinder heave with the same amplitude and frequency. Hence, we must consider appropriate motions for both cylinder and foil concurrently in the choice of experiments. The cylinder performs best at double amplitudes between one and three diameters and at frequencies where $\pi S t_{d} \approx 1$. The foil performs well over a variety of frequencies and amplitudes with the angle of attack dominating the performance. So, we began by specifying a good vortex generator: amplitude ratio $\frac{2 A}{d}=2.0$ and $S t_{d}=0.25$. This gives foil parameters $\frac{2 A}{c}=1.0$ and $S t_{A}=0.50$ which are acceptable foil motions for good vortex and jet wake production.

Figure 5-7 and 5-8 show the DPIV velocity and vorticity data for this vortex generator (case vc1) without the foil present as viewed in the inertial frame. The wake is wide and consistent with average width $h=3.6 d$ and lateral spacing between same side vortices $\frac{s}{\lambda}=0.70$. The average velocity defect is $0.87 \mathrm{U}$ and the average convection speed of the vortices is $0.27 \mathrm{U}$. The drag coefficient, normalized with respect to the foil chord here instead of the cylinder diameter, is equal to 2.9 .

We investigated the interaction of the D-cylinder wake with two foil motions which have pitch angles $\theta_{\circ}=30^{\circ}$ and $15^{\circ}$ and constant phase angle $\phi=90^{\circ}$. The maximum angles of attack for these motions without considering the effect of the cylinder wake are $28^{\circ}$ and $43^{\circ}$ respectively. Figures 5-9 through 5-12 show the velocity and vorticity fields for these cases of the foil alone. In the lower angle of attack case ( $v c 2)$, the wake is typical of the results of Chapter 4. A small dynamic stall vortex (DSV) forms and immediately merges with the same signed trailing edge vorticity (TEV) forming a reverse Kármán street. The vortices are well formed and persist for several wavelengths of the motion. This motion generates large thrust $\left(C_{f}=3.3\right)$ because of the high angle of attack and Strouhal number.

In the higher angle of attack case (vc8), the wake strengthens as expected. The DSV is much more energetic and does not immediately merge into the TEV as in the previous 


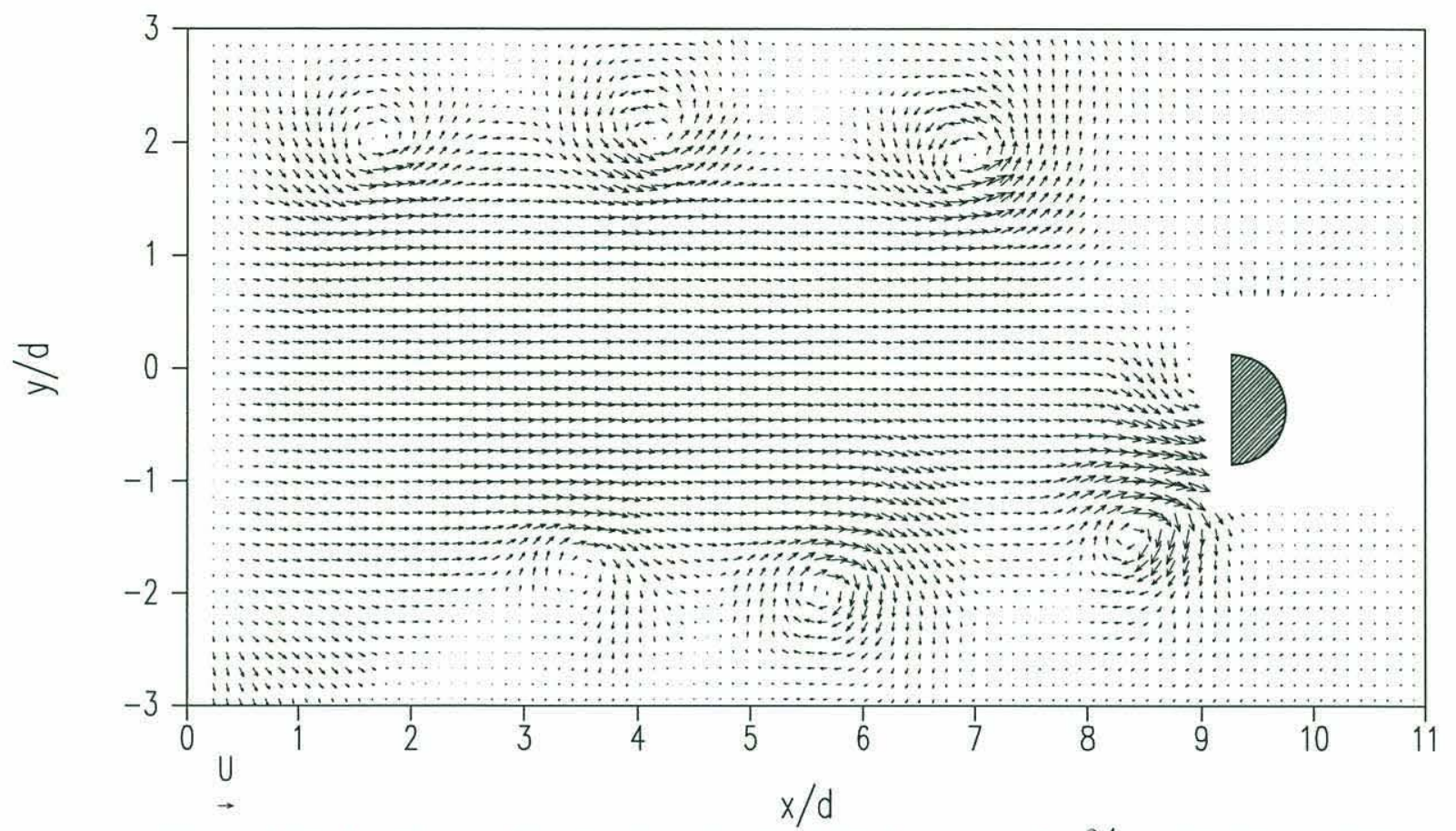

Figure 5-7: Oscillating D-cylinder velocity field for case vc1. $\frac{2 A}{d}=2.0, S t_{d}=0.25$.

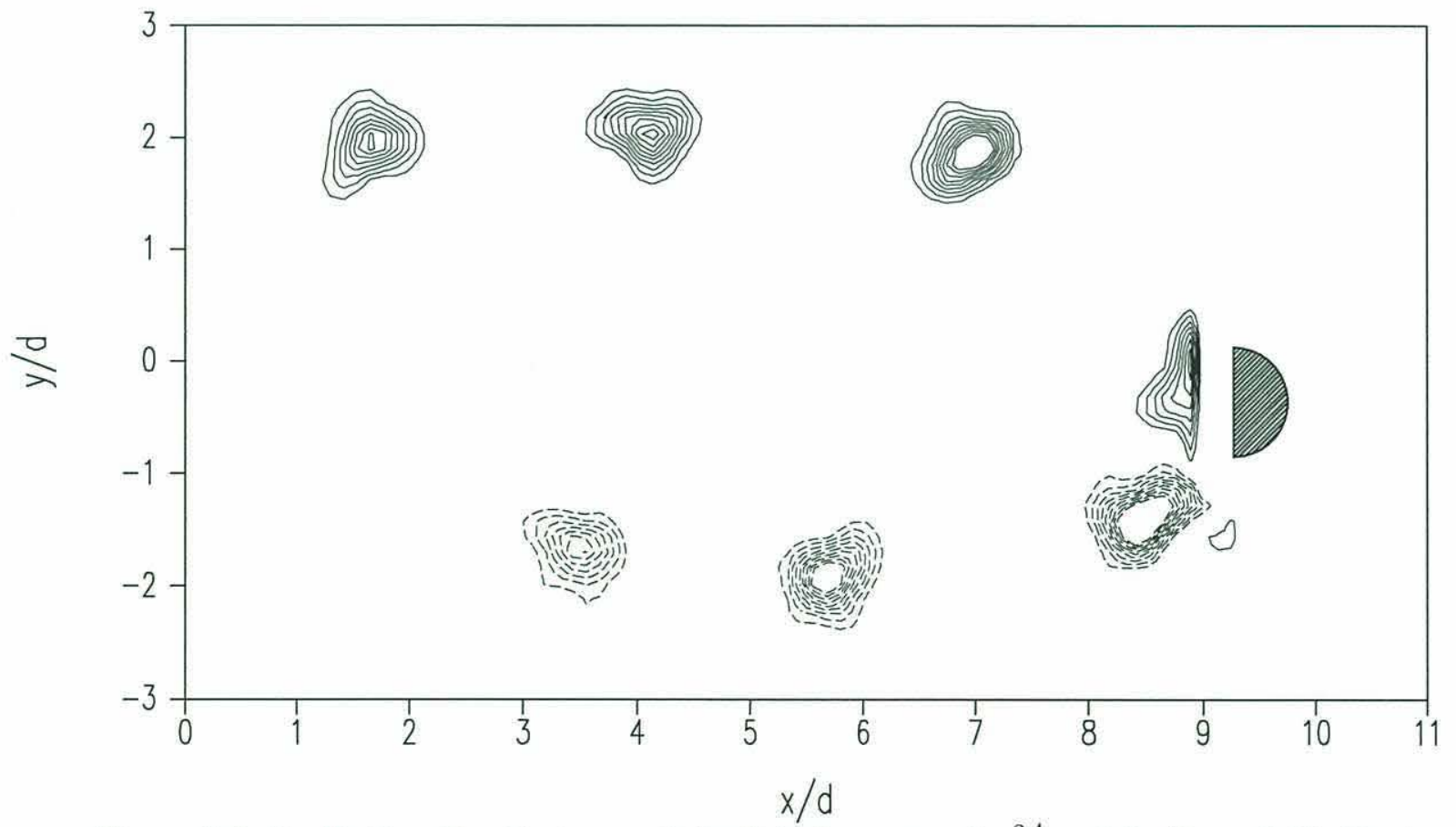

Figure 5-8: Oscillating D-cylinder vorticity field for case vc1. $\frac{2 A}{d}=2.0, S t_{d}=0.25$. Contour levels are from -10.0 to 10.0 in $1.0 \frac{1}{s}$ increments excluding the zero contour. 


\begin{tabular}{|c|c|c|c|c|c|c|c|c|}
\hline case & $\begin{array}{l}\theta_{0} \\
\left({ }^{\circ}\right)\end{array}$ & $\frac{\sigma}{\lambda}$ & $C_{f}$ & $\bar{\Gamma}^{*}$ & $\frac{u-U}{U}$ & $\frac{h}{c}$ & $\begin{array}{c}\text { interaction } \\
\text { mode }\end{array}$ & $\begin{array}{c}\text { wake } \\
\text { interaction }\end{array}$ \\
\hline $\begin{array}{c}v c 1 \\
\text { (cyl only) } \\
\text { vc2 } \\
\text { (foil only) }\end{array}$ & 30 & - & $\begin{array}{l}-2.9 \\
3.3\end{array}$ & $\begin{array}{l}1.5 \\
2.2\end{array}$ & 0.77 & $\begin{array}{l}1.80 \\
0.89\end{array}$ & - & - \\
\hline $\begin{array}{l}\text { vc3 } \\
\text { vc4 } \\
\text { vc5 } \\
\text { vc6 } \\
\text { vc7 }\end{array}$ & $\begin{array}{l}30 \\
30 \\
30 \\
30 \\
30\end{array}$ & $\begin{array}{l}1.00 \\
1.25 \\
1.50 \\
0.75 \\
0.50\end{array}$ & $\begin{array}{l}2.5 \\
1.9 \\
2.7 \\
1.3 \\
1.3\end{array}$ & $\begin{array}{l}3.1 \\
2.8 \\
2.6 \\
2.6 \\
2.5 \\
\end{array}$ & $\begin{array}{l}0.83 \\
0.87 \\
0.69 \\
0.68 \\
0.47\end{array}$ & $\begin{array}{l}0.37 \\
0.38 \\
0.37 \\
0.45 \\
0.42\end{array}$ & $\begin{array}{c}\text { interception } \\
\text { slalom } \\
\text { combination } \\
\text { interception } \\
\text { slalom }\end{array}$ & $\begin{array}{l}t_{1}, b_{1} \\
t_{3}, b_{3} \\
t_{3}, b_{1} \\
t_{1}, b_{1} \\
t_{3}, b_{3}\end{array}$ \\
\hline $\begin{array}{c}\text { vc8 } \\
\text { (foil only) }\end{array}$ & 15 & - & 3.8 & 2.9 & 1.11 & 0.92 & - & - \\
\hline $\begin{array}{l}\text { vc9 } \\
\text { vc10 } \\
\text { vc11 } \\
\text { vc12 }\end{array}$ & $\begin{array}{l}15 \\
15 \\
15 \\
15\end{array}$ & $\begin{array}{l}1.00 \\
1.25 \\
1.50 \\
0.75\end{array}$ & $\begin{array}{l}2.8 \\
2.4 \\
1.8 \\
1.8\end{array}$ & $\begin{array}{l}3.9 \\
3.5 \\
3.3 \\
3.9\end{array}$ & $\begin{array}{l}1.04 \\
1.08 \\
0.82 \\
0.96\end{array}$ & $\begin{array}{l}1.14 \\
0.42 \\
0.72 \\
0.60\end{array}$ & $\begin{array}{l}\text { interception } \\
\text { slalom } \\
\text { combination } \\
\text { interception }\end{array}$ & $\begin{array}{l}t_{2}, b_{2} \\
t_{3}, b_{3} \\
t_{4}, b_{4} \\
t_{4}, b_{2}\end{array}$ \\
\hline
\end{tabular}

Table 5.2: Vortex control experiments at $S t_{A}=0.50, \frac{2 A}{c}=1.0$ and $\phi=90^{\circ}$. The spacing between the foil pivot point and the cylinder center $\sigma$ is normalized by the wavelength of the motion $\lambda=\frac{U}{f}$. Interaction structure for top ('t') and bottom ('b') row cylinder vortices are indicated with subscripts: 1 - annihilation by stretching, 2 - constructive merging with foil dynamic stall vortex (DSV), 3 - constructive merging with same signed trailing edge vorticity (TEV) and 4 - destructive merging with oppositely signed shear layer.

case. The same signed DSV and TEV rotate about one another while merging in the near wake. This is shown clearly in the vorticity contours in Figure 5-12. The two vortices merge quickly into a single vortex in less than a half a period of the motion. The thrust coefficient increases to 3.8 which is a modest gain considering the large increase in angle of attack.

Table 5.2 summarizes the experimental space and results for the tandem cylinder-foil experiments. The vortex encounter phasing was adjusted by varying the spacing, $\sigma$, between the foil pivot point and the cylinder center. The spacing was varied between $0.5 \lambda$ and $1.5 \lambda$ in $0.25 \lambda$ increments where $\lambda$ is the wavelength of the motion in space. When the spacing is $0.5 \lambda$ or less, the foil begins to suppress the cylinder shedding. Cases vc3 through vc7 correspond to the lower angle of attack foil motion and cases vc9 through vc12 to the high angle of attack motion.

Our goal in these experiments is to study the interactions between a flapping foil and oncoming discrete vortices. Ideally, we would like to superpose the flow field of the cylinder alone and the foil alone and observe the interactions. Unfortunately, the presence of the foil 
suppresses cylinder shedding and the cylinder wake alters the inflow conditions to the foil, effectively increasing the apparent angle of attack. Another significant complication is the fact that the cylinder wake streamwise wavelength $s$ is not equal to the motion wavelength $\lambda$. In other words, the encounter frequency of the cylinder vortices does not equal the foil motion frequency which causes the foil to encounter top row cylinder vortices differently than bottom row vortices. Consequently, different wake interactions occur. We consider this fortuitous, because it enabled us to observe a wider variety of encounters and interactions.

\subsubsection{Results and discussion}

The value of $\sigma$ determines the interaction mode by controlling how the foil encounters the cylinder vortices. For $\sigma=0.75$ and 1.00 , the foil comes very close to the cylinder vortices and we refer to this as an interception mode. Alternatively, when $\sigma=0.50$ or 1.25 , the foil avoids the vortices in a slalom mode. Because of the wavelength mismatch between the cylinder wake and the motion, combination modes also result where close encounters happen in one row while in the other row the encounter is more like the slalom mode. Again, this variety of encounter scenarios is not necessarily a hindrance to our experiments since we observed a greater variety of interactions.

The foil is fixed to the cylinder at a specified distance in the wake, which guarantees that the foil will always encounter each row of vortices in the same manner each cycle. The cylinder wake is periodic and locked onto the motion. Thus, the observed interactions are periodic and repeatable despite the wavelength mismatch.

Figures 5-13 through 5-21 summarize the flow and interaction for the cases listed in Table 5.2. The flow summaries contain four velocity plots starting at the point of maximum heave proceeding in increments of one quarter of the motion cycle. The abscissae are adjusted such that the foil pivot is fixed at $\frac{x}{c}=0$. The DPIV data view does not usually include the flow between the cylinder and foil. In addition, shadowed regions sometimes obscure the cylinder vortices and are removed using the standard method. The velocity fields are presented in the inertial reference frame such that the wake vortices are well resolved.

Schematic diagrams of the wake structure and subsequent interaction of vortices are shown alongside the velocity plots. The schematic diagrams show vortices not clearly visible in the velocity plots due to the limited data view and optical contaminations such as shadows 


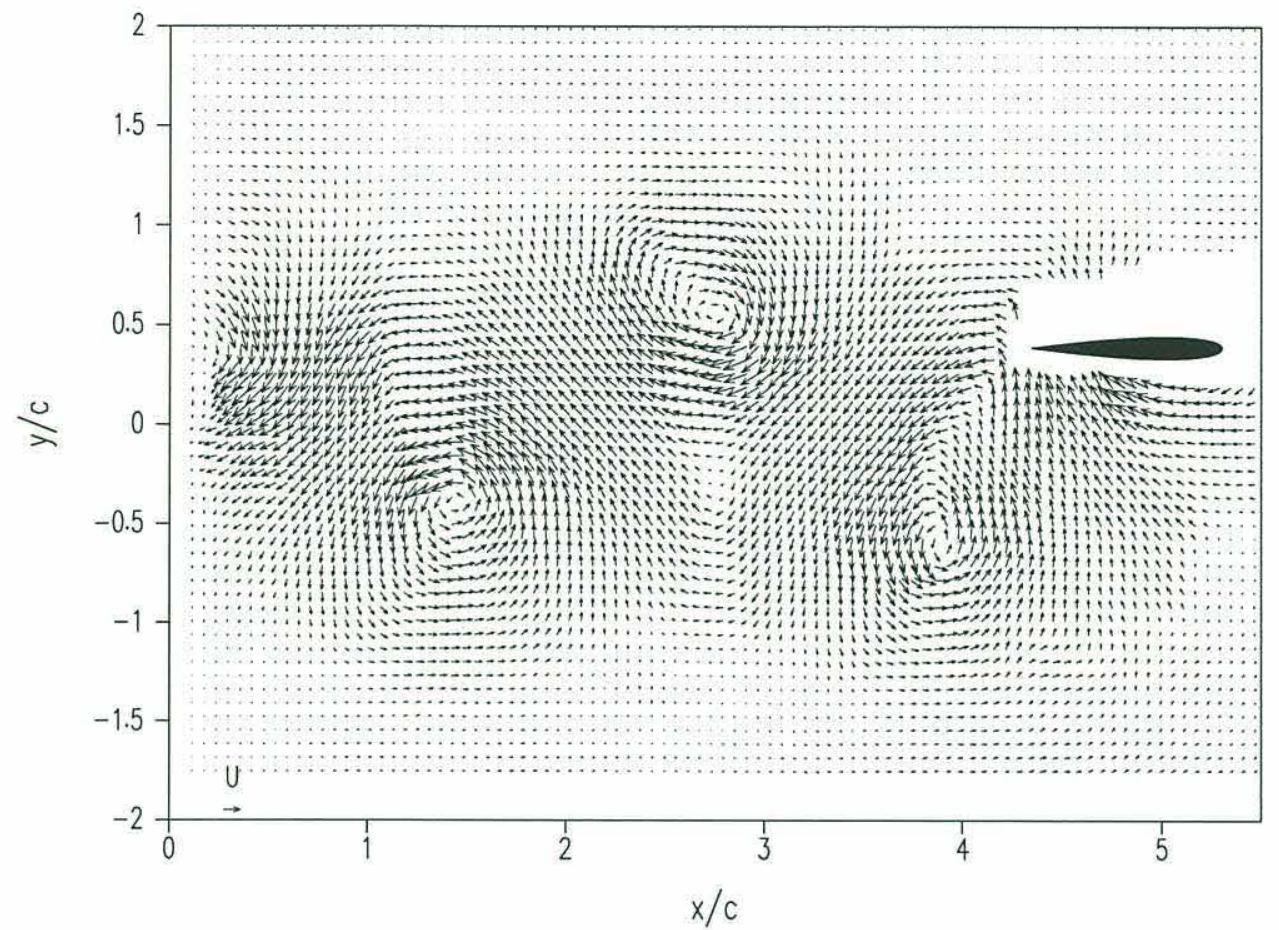

Figure 5-9: DPIV velocity field for foil only, case vc2. Foil at maximum heave position. $S t_{T E}=0.86, \frac{2 A}{c}=1.0, \theta_{\circ}=30, \alpha_{\max }=27.7^{\circ}, \phi=90^{\circ}$.

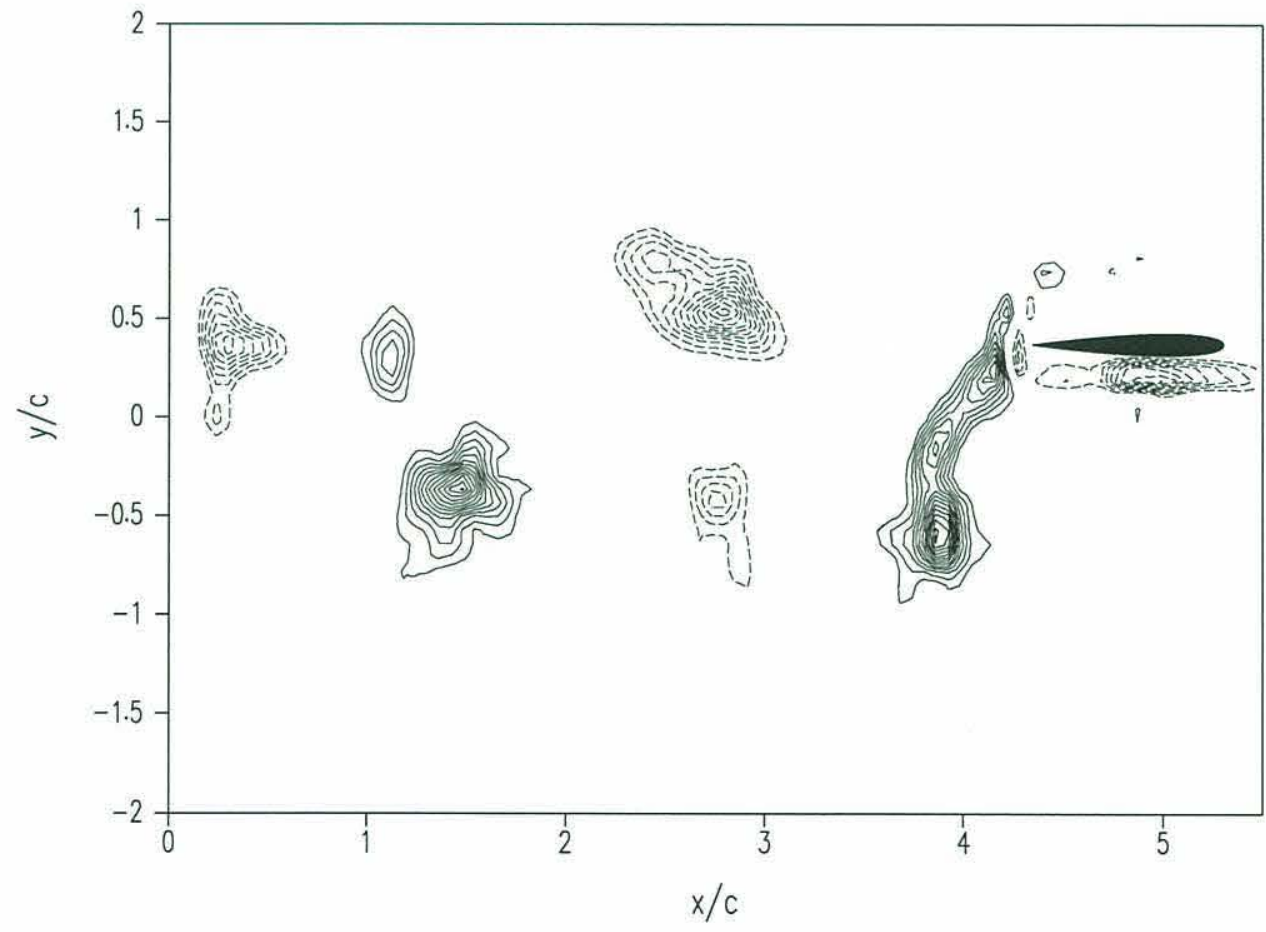

Figure 5-10: DPIV vorticity field for foil only, case vc2. Foil at maximum heave position. Contour levels are from -20.0 to $20.0 \frac{1}{s}$ in $1.0 \frac{1}{s}$ increments excluding the zero contour. 


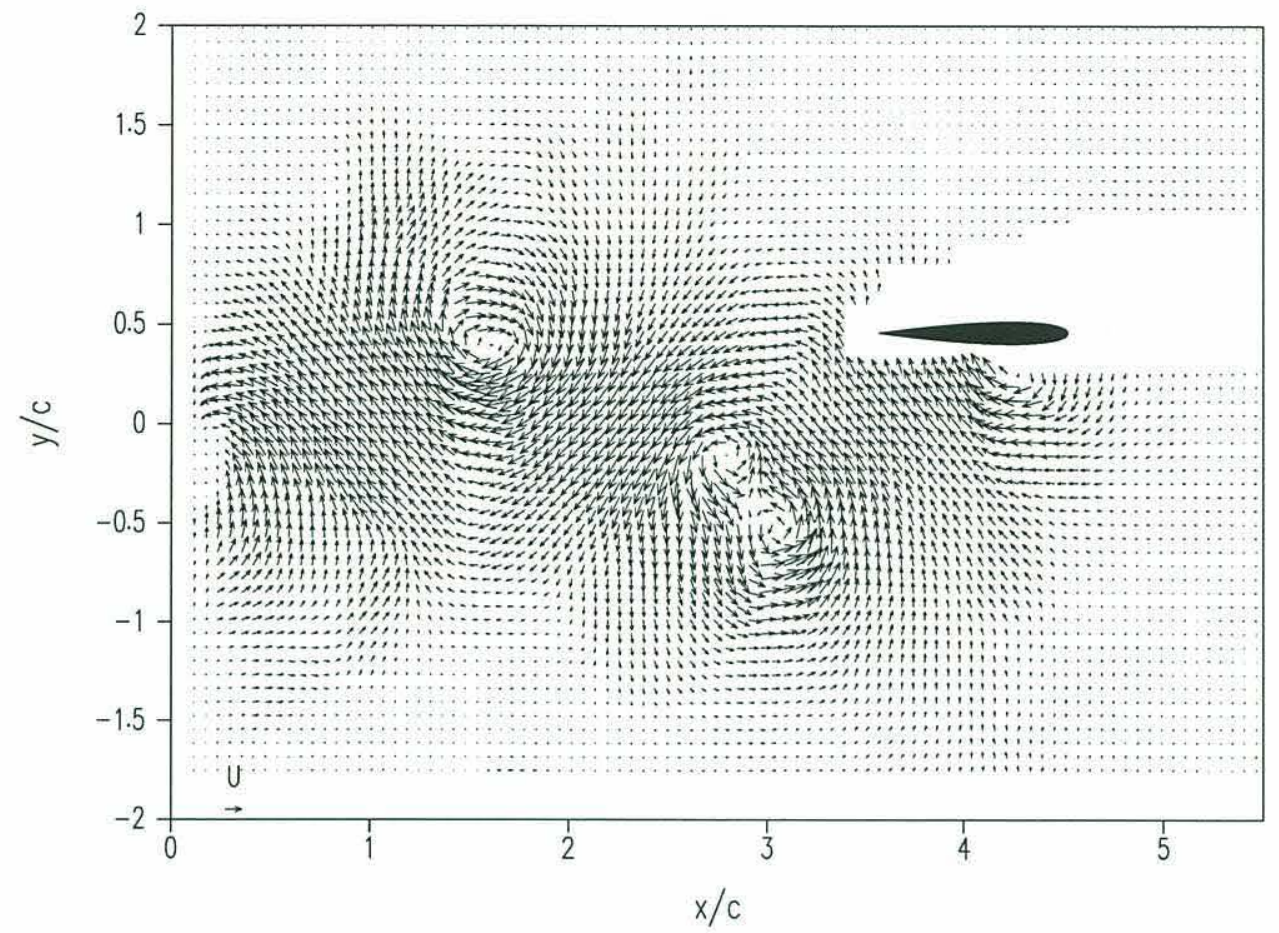

Figure 5-11: DPIV velocity field for foil only, case vc8. Foil at maximum heave position. $S t_{T E}=0.61, \frac{2 A}{c}=1.0, \theta_{\circ}=15, \alpha_{\max }=42.5^{\circ}, \phi=90^{\circ}$.

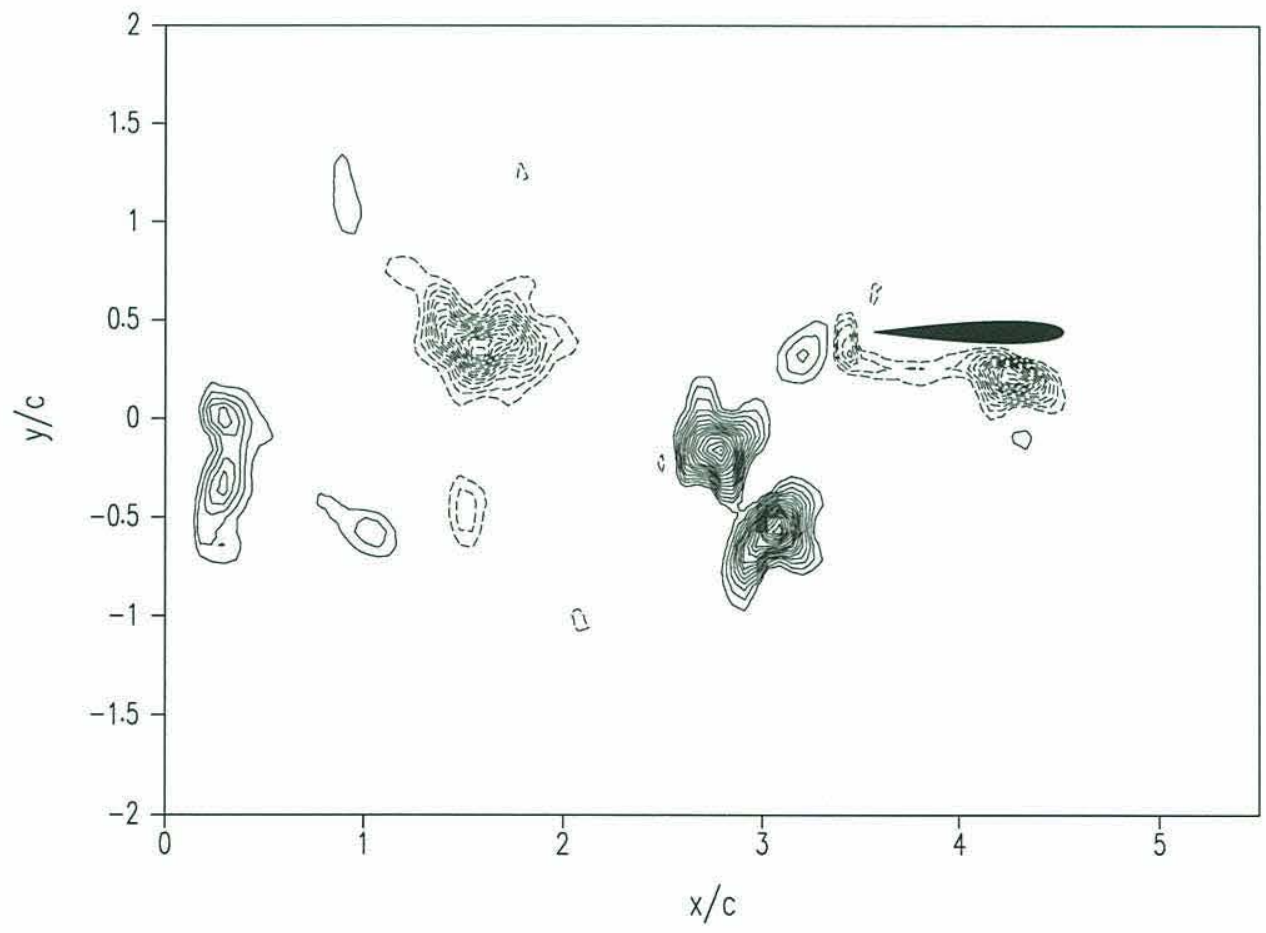

Figure 5-12: DPIV vorticity field for foil only, case vc8. Foil at maximum heave position. Contour levels are from -20.0 to $20.0 \frac{1}{s}$ in $1.0 \frac{1}{s}$ increments excluding the zero contour. 
and reflections. The locations of the obscured vortices were ascertained by careful viewing of the raw video data. Cylinder vortices are labeled with roman numerals from oldest to newest. Foil vortices are labeled with capital letters following the same convention. Foil stall vortices are labeled with lower case letters which correspond to the upper case labeled vortex with which the stall vortex will merge in the near wake.

Figure 5-13 summarizes the flow for case vc3 where the angle of attack is moderate and the separation is one motion wavelength. The foil closely encounters the cylinder vortices in an interception mode: top row vortices come near the foil leading edge and the bottom row vortices hit the foil at approximately the mid-chord. Clearly, the foil motion dominates the formation of a reverse Kármán street. The oncoming cylinder vortices are annihilated by straining due to the motion of the foil. In Figure 5-13, when the foil is at the position of maximum heave, cylinder vortex (i) is very close to the foil leading edge. As the foil moves downward, the vortex is strained and disappears quickly. Similarly, cylinder vortex (ii) disintegrates during the foil upstroke.

The flow around the foil is perturbed by the cylinder vortices yet forms essentially the same vortical structures as in the foil alone case (Figure 5-9). There is a $24 \%$ reduction in the thrust coefficient although the wake vortex circulation increases by $41 \%$. This result is unexpected since the cylinder wake increases the effective angle of attack that the foil feels and should increase the net thrust. The reduced thrust is due to the alignment of the vortices in the wake closer to the centerline. The vortex strength and wake excess velocity are both larger than in the foil only case, yet there is nearly a $60 \%$ reduction in the near wake width. Thus, the overall thrust is reduced.

In comparison to the foil alone case, the dynamic stall vortex increases in strength, likely due to the increase in angle of attack. The increased DSV strength indicates that the downwash from the near cylinder vortices does not significantly inhibit DSV formation. By the time the foil comes near the cylinder vortices, the DSV is fully developed and convecting downstream.

Figure 5-14 summarizes the flow for the vc4 case where the separation between the cylinder and foil is increased to $1.25 \lambda$. Here, the foil avoids the cylinder vortices in a slalom mode. In the maximum heave position, we see the foil preparing to move downward between cylinder vortices (i) and (ii). During the downstroke, the stall vortex (d) moves along the foil surface to join with forming TEV (D). Cylinder vortex (i) moves upward 

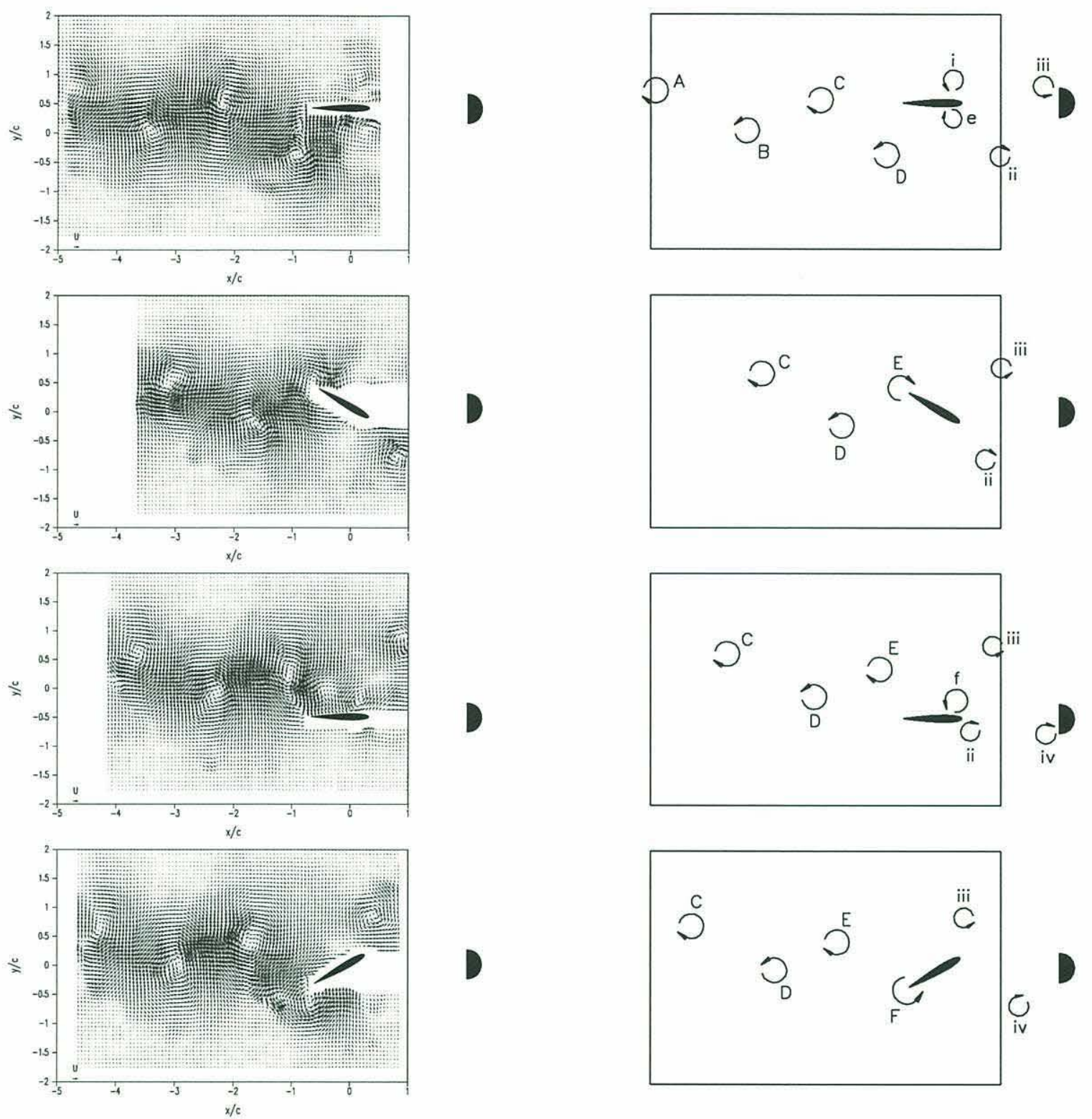

Figure 5-13: Flow summary for vc3 case, $\frac{\sigma}{\lambda}=1.00$, interception mode with annihilation of both rows of cylinder vortices by stretching. Velocity fields at quarter points in the heave cycle are shown in the left column, schematic diagrams of the wake vortices in the right column. The abscissa is adjusted such that the foil pivot point is at $\frac{x}{c}=0$. 
following (d) and also merges with the same signed TEV (D). Meanwhile, cylinder vortex (ii) from the top row moves downward, and merges with TEV (E) along with the stall vortex (e). The top row cylinder vortices merge with the TEV while it is forming whereas the bottom row vortices merge with TEV in the near wake. This case illustrates two different types of constructive merging which depend on the proximity of the cylinder vortex to the foil. The foil suction can either draw the cylinder vortex near the foil surface and into the forming TEV or draw it into the wake where it merges with TEV.

In this interaction, as with the previous, the cylinder wake is completely reformed by the foil motion. A reverse Kármán street forms with diminished wake width and increased strength similar to the previous case. The circulation of the near wake vortices is larger than the foil alone vortices by $27 \%$ which supports the notion of constructive merging. However, the result is not markedly different from the previous case where vortices are clearly annihilated and not constructively merged. This apparent discrepancy is likely due to the error in the circulation measurement in a highly mixed wake. Recall from Chapter 4 , the error in the circulation calculation was typically less than $5 \%$ for well behaved wakes. However, when the wake is relatively mixed as in these cases, the typical standard deviations are 10 to $15 \%$. Thus, we must qualify our interpretation of the circulation data in these interaction wakes.

Case vc5 is summarized in Figure 5-15. Here, $\frac{\sigma}{\lambda}=1.50$ which results in a combined interception and slalom mode. Top row cylinder vortices are avoided and constructively merged while bottom row vortices are intercepted and annihilated by stretching. At the point of maximum heave we see cylinder vortex (i) at the trailing edge of the foil. As the foil moves downward, (i) moves into the wake where it begins merging with same signed vortex (D). Meanwhile, cylinder vortex (ii) closely intercepts the foil and is destroyed by stretching. The interactions in this case essentially match those observed in the interception mode of case vc3 and the slalom mode of case vc4. The wake width, thrust coefficient and vortex strength differ similarly as previous cases from the foil alone case.

Figure 5-16 shows the results for case vc6 where the separation has been reduced to $0.75 \lambda$. In this case, the interaction is an interception mode with the foil encountering the top row vortices near the trailing edge and the bottom row vortices near the mid-chord. During the downstroke, top row cylinder vortex (i) interacts with the formation of TEV (E): (i) is pushed upward and then strained to destruction. In the bottom row, cylinder 

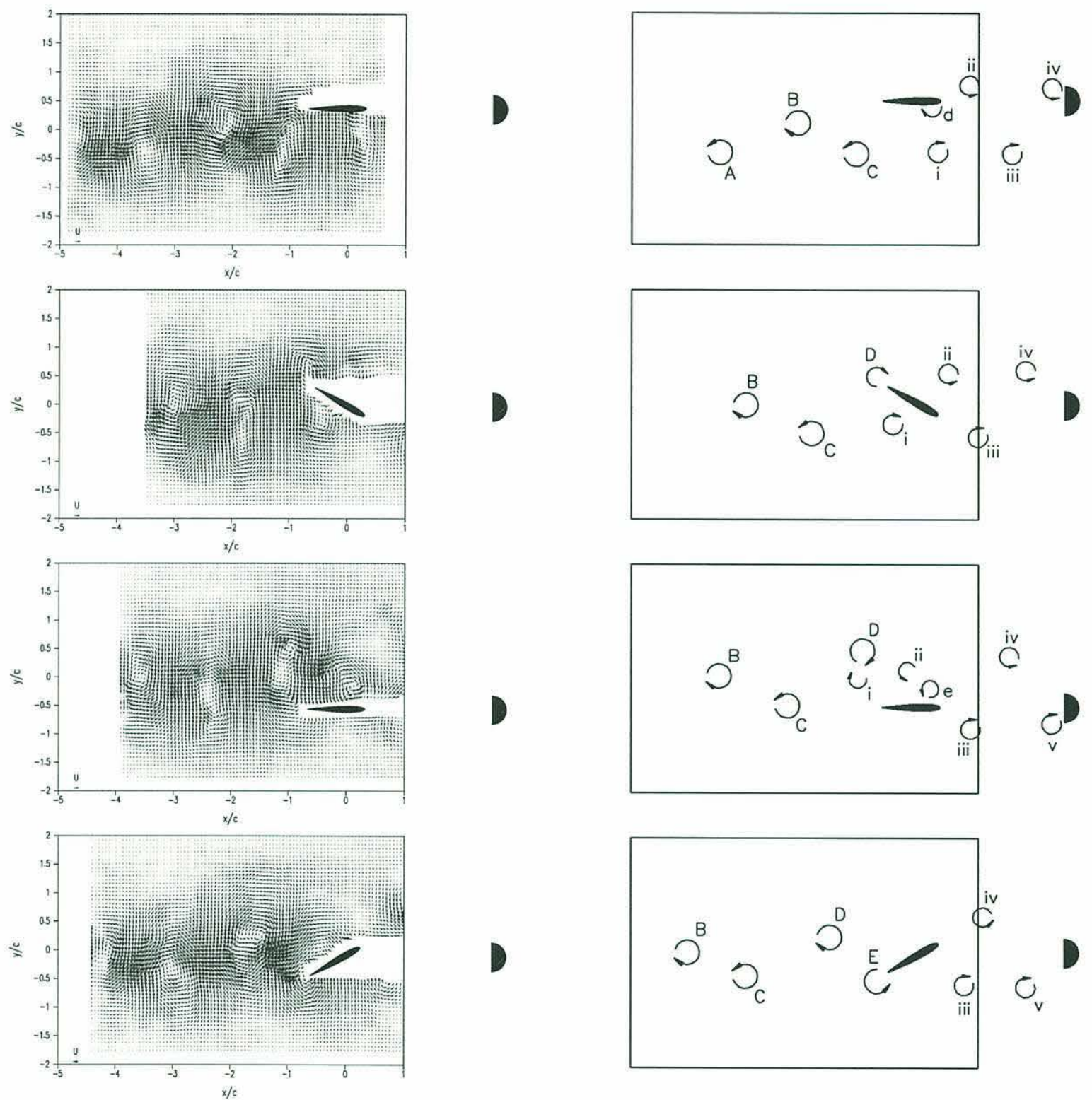

Figure 5-14: Flow summary for vc4 case, $\frac{\sigma}{\lambda}=1.25$, slalom mode with constructive merging of both rows of cylinder vortices with TEV. Velocity fields at quarter points in the heave cycle are shown in the left column, schematic diagrams of the wake vortices in the right column. The abscissa is adjusted such that the foil pivot point is at $\frac{x}{c}=0$. 

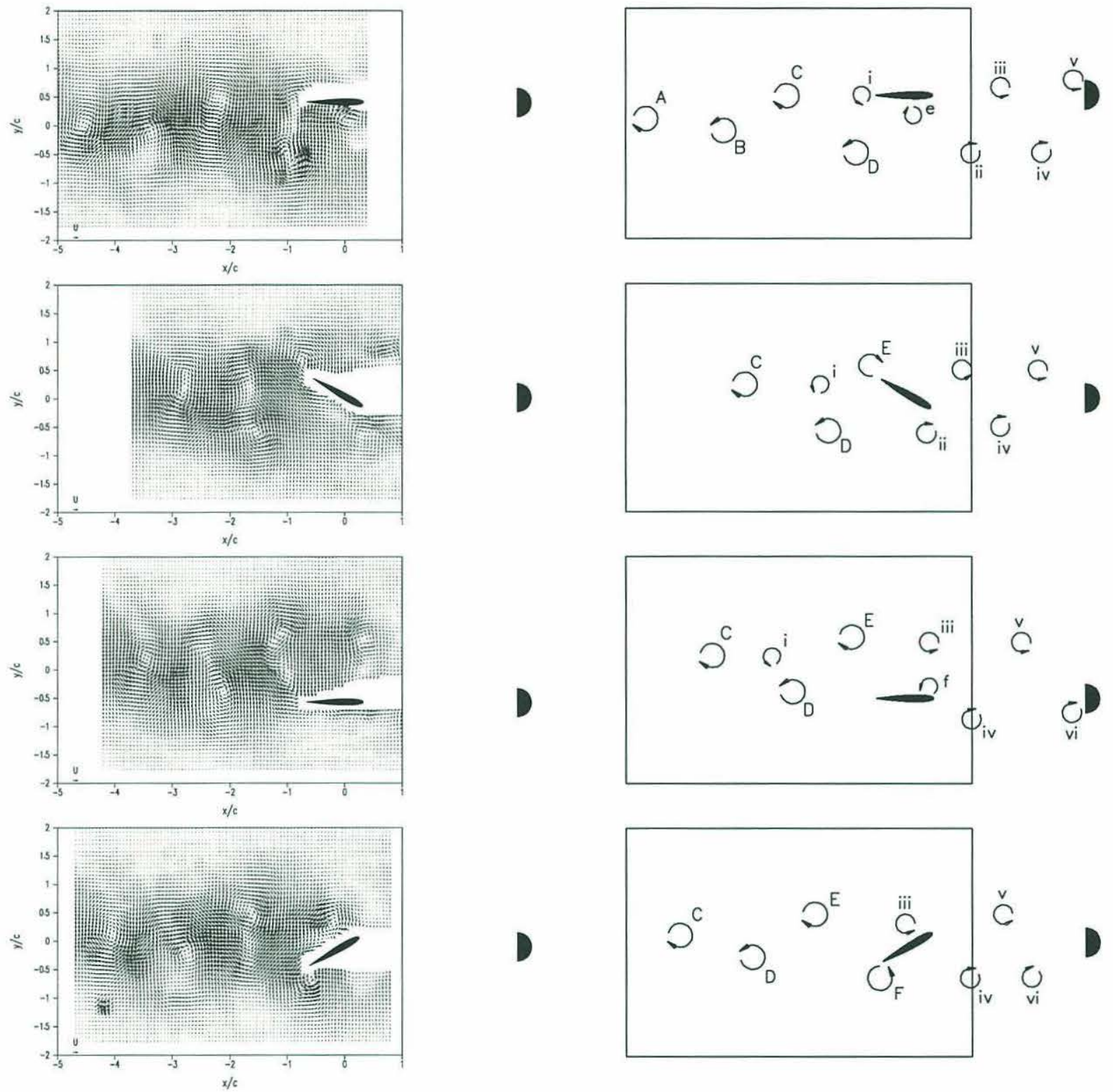

Figure 5-15: Flow summary for vc5 case, $\frac{\sigma}{\lambda}=1.50$, combination interception and slalom mode with annihilation by stretching of bottom row cylinder vortices and constructive merging of top row vortices with TEV. Velocity fields at quarter points in the heave cycle are shown in the left column, schematic diagrams of the wake vortices in the right column. The abscissa is adjusted such that the foil pivot point is at $\frac{x}{c}=0$. 
vortex (ii) is lifted by the foil slightly and stretched to destruction during the upstroke.

Case vc7 is summarized in Figure 5-17. In this case, the foil follows the cylinder so closely that it begins to interfere with the shedding process. The swinging of the flow around the cylinder base is prevented by the presence of the foil, resulting in weaker shedding. Zdravkovich [147] presents an excellent review of this and other vortex suppression devices. It is clearly undesirable for us to suppress cylinder shedding for our studies so we did not repeat this case for the higher angle of attack foil motion. Despite the reduced cylinder vortex strength, a slalom interaction similar to case vc4 occurs. Both top and bottom row vortices constructively merge with same signed TEV.

Figure 5-18 through 5-21 show the interaction cases for vc9 through vc12 where the foil angle of attack is nearly doubled. In these cases, the interaction modes for a given separation are the same as observed previously, yet the wake interactions are distinctly different.

Figure 5-18 shows case vc9 where $\frac{\sigma}{\lambda}=1.00$, which produces an interception mode. Previously, in the low angle of attack case, the foil annihilated the cylinder vortex street by stretching. In this case, the cylinder vortices attach to and follow the foil surface, constructively merging with the foil stall vortex. Bottom row vortices are encountered very near the foil leading edge and top row vortices just upstream of the mid-chord as in case vc3. In Figure 5-18, cylinder vortex (ii) from the top row follows the foil downward, either merging with or replacing the stall vortex, and eventually merging with TEV (E). Similarly, cylinder vortex (i) joins the foil surface during the upstroke and eventually merges into TEV (D).

The circulation of the wake vortices increases by $34 \%$ compared to the foil alone case. This increase is sufficiently large enough (compared to the circulation calculation error) to support the conclusion that cylinder vorticity is either transferred into the trailing edge vortices or creates sufficient downwash to increase the strength of the TEV. The foil restructures an unfavorable drag wake into a favorable thrust wake by repositioning the drag vortices such that they will contribute to a jet wake formation.

Figure 5-19 illustrates case vc10 where there is a slalom mode interaction. In the low angle of attack case (vc4) for this separation, both rows of cylinder vortices constructively merge with trailing edge vorticity. In this case, we see essentially the same result. In Figure 5-19, cylinder vortex (i) merges with TEV (D) at the trailing edge while cylinder vortex (ii) follows the foil down, merging with TEV (E). 

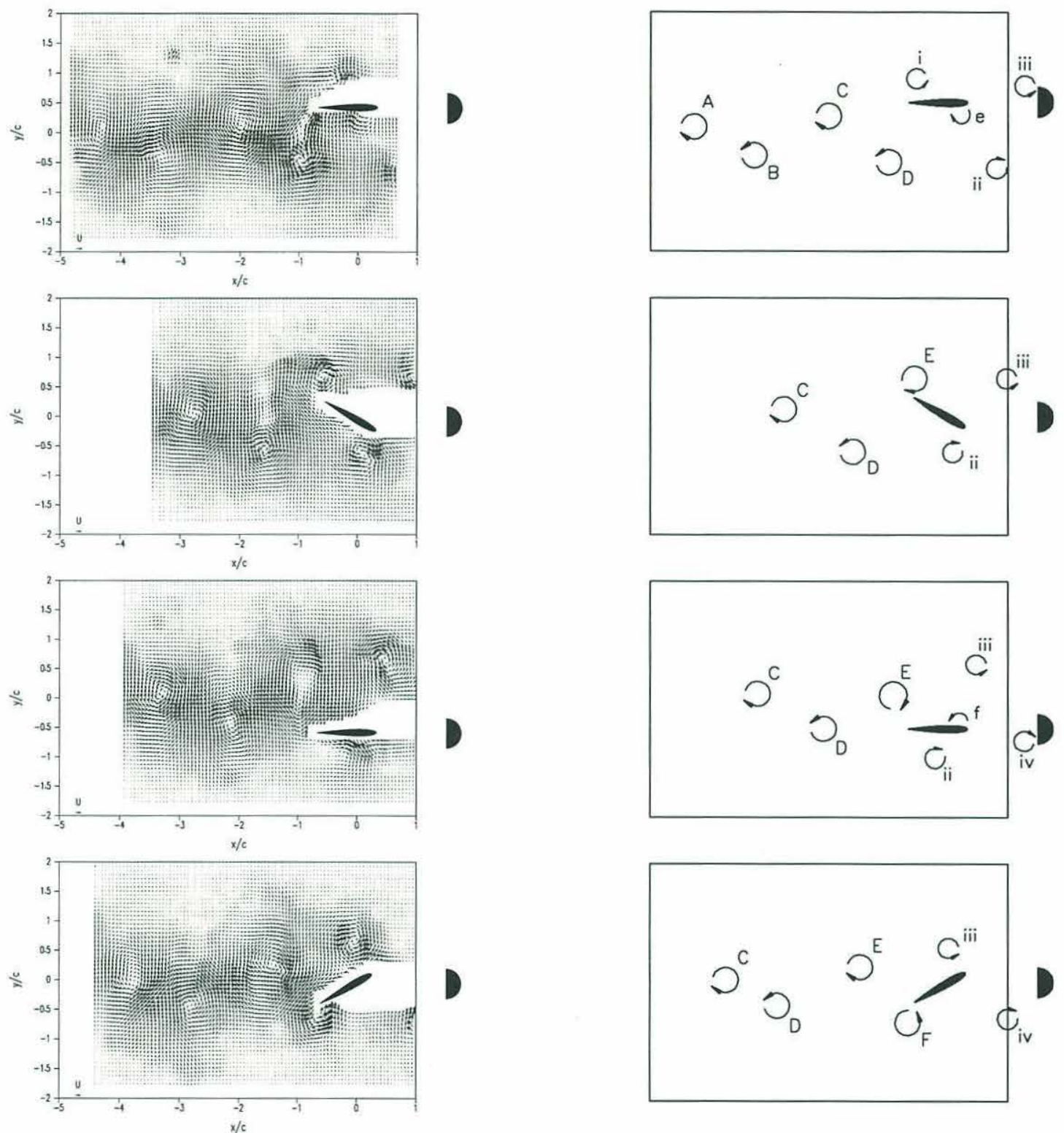

Figure 5-16: Flow summary for vc6 case, $\frac{\sigma}{\lambda}=0.75$, interception mode with annihilation by stretching of both rows of cylinder vortices. Velocity fields at quarter points in the heave cycle are shown in the left column, schematic diagrams of the wake vortices in the right column. The abscissa is adjusted such that the foil pivot point is at $\frac{x}{c}=0$. 

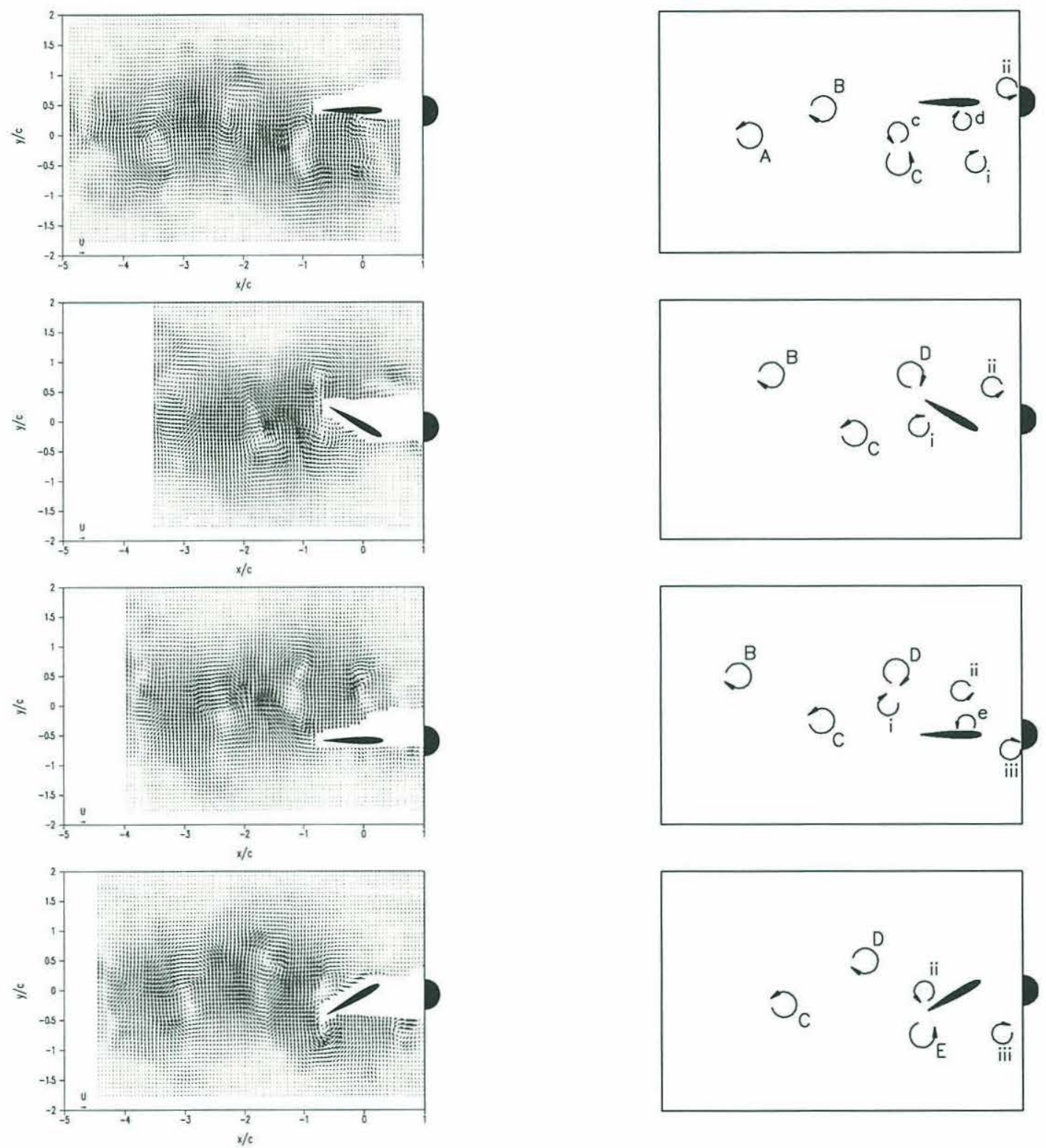

Figure 5-17: Flow summary for vc7 case, $\frac{\sigma}{\lambda}=0.50$, slalom mode with constructive merging of both cylinder vortex rows with TEV. Velocity fields at quarter points in the heave cycle are shown in the left column, schematic diagrams of the wake vortices in the right column. The abscissa is adjusted such that the foil pivot point is at $\frac{x}{c}=0$. 

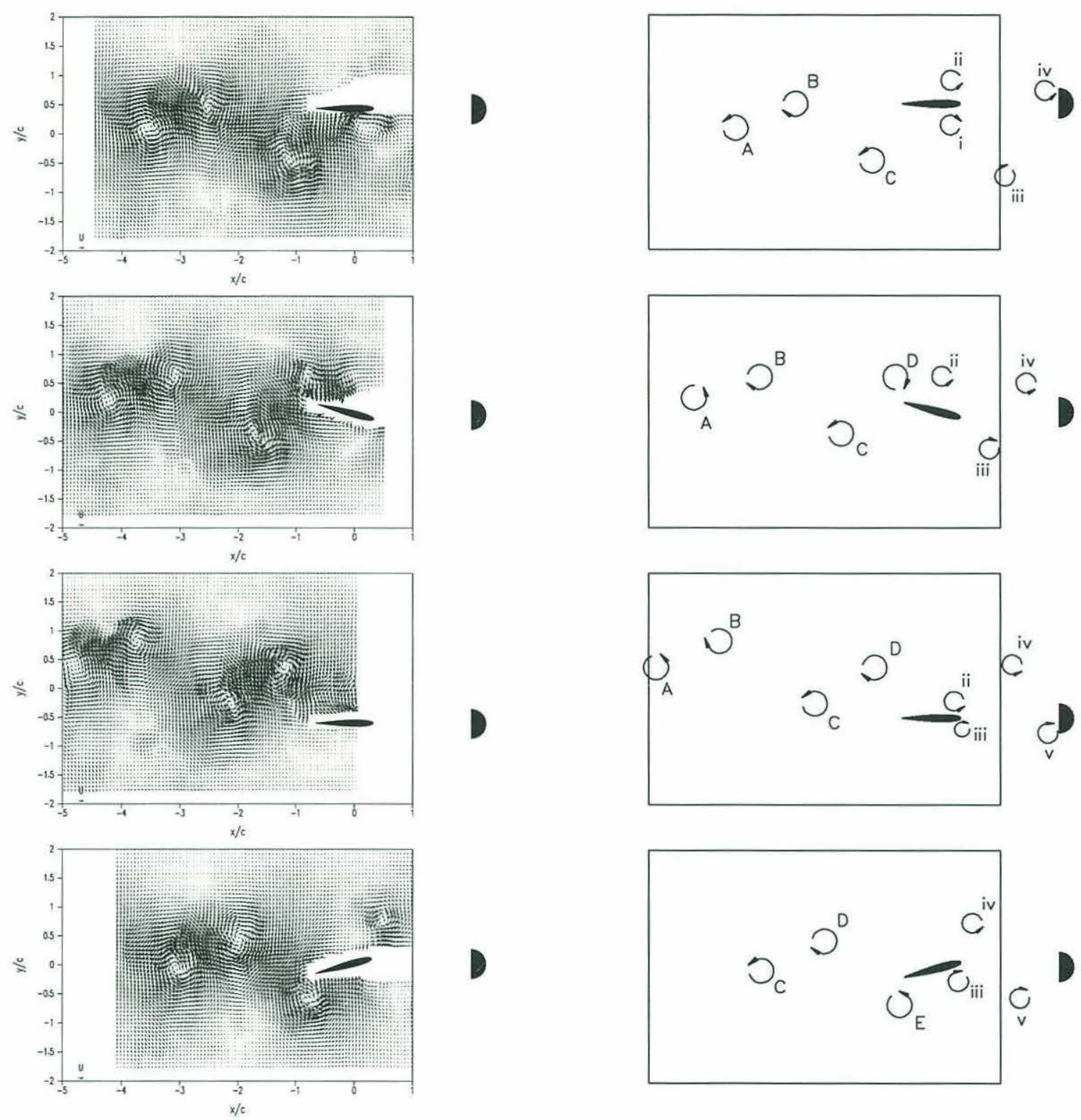

Figure 5-18: Flow summary for vc9 case, $\frac{\sigma}{\lambda}=1.00$, interception mode with constructive merging of both rows of cylinder vortices with the DSV. Velocity fields at quarter points in the heave cycle are shown in the left column, schematic diagrams of the wake vortices in the right column. The abscissa is adjusted such that the foil pivot point is at $\frac{x}{c}=0$. 
Contrary to the low angle of attack case, the stall vortex for this motion does not convect and contribute to the trailing edge vortices as previously. Stall vortices (d) and (e) appear and then are quickly suppressed, which is likely due to the extremely high rate of change of the angle of attack. In our foil studies in Chapter 4 we noted that extreme pitch rates $\Omega^{*}$ can suppress leading edge vortices even after they are formed. In this case, the combination of the nominally high angle of attack and the increase from the deficit cylinder wake quash the leading edge vortex before it convects towards the trailing edge. This stall vortex suppression does not occur in case vc9 because the merging cylinder vortices energize and aid in convecting the stall vortex towards the trailing edge.

Figure 5-20 shows the combination interaction mode of case vc11. The foil intercepts bottom row vortices at the mid-chord and avoids the top row vortices (the interaction is at the trailing edge). Cylinder vortices from both rows are destroyed by interaction with the shear layer emanating from the trailing edge. They stretch and disappear as the TEV forms. Again, the stall vortices are suppressed by the foil motion and do not contribute to the wake. This case differs from the corresponding low angle of attack case in that the bottom row vortices do not convect past the forming TEV to merge with the previously shed same signed vorticity. In this case, the trailing edge shear layer traps the cylinder vortex and annihilates it by stretching.

Case vc12 is illustrated in Figure 5-21. The foil intercepts the top row slightly downstream of the mid-chord and the bottom row slightly upstream of the trailing edge. The top row vortices are drawn towards the trailing edge and then destroyed by interaction with the shedding shear layer. Similar to case vc9, the bottom row cylinder vortex joins with the same signed stall vortex, convects and constructively merges with shed trailing edge vorticity.

Figure 5-22 summarizes the variation in thrust coefficient as a function of the separation (phasing). The data are plotted normalized with respect to the thrust coefficient of the corresponding foil only case for each group of experiments. The net force ranges between 40 and 80 percent of the tare value, depending on the type of interaction mode. In both angle of attack cases, the slalom mode diminishes the net thrust, and the interception mode increases the thrust. In the interception mode, the foil is more heavily loaded and produces greater thrust. In the slalom mode, cylinder vortices are always constructively merged with TEV, yet interestingly, the net force is reduced compared to the interception mode. 

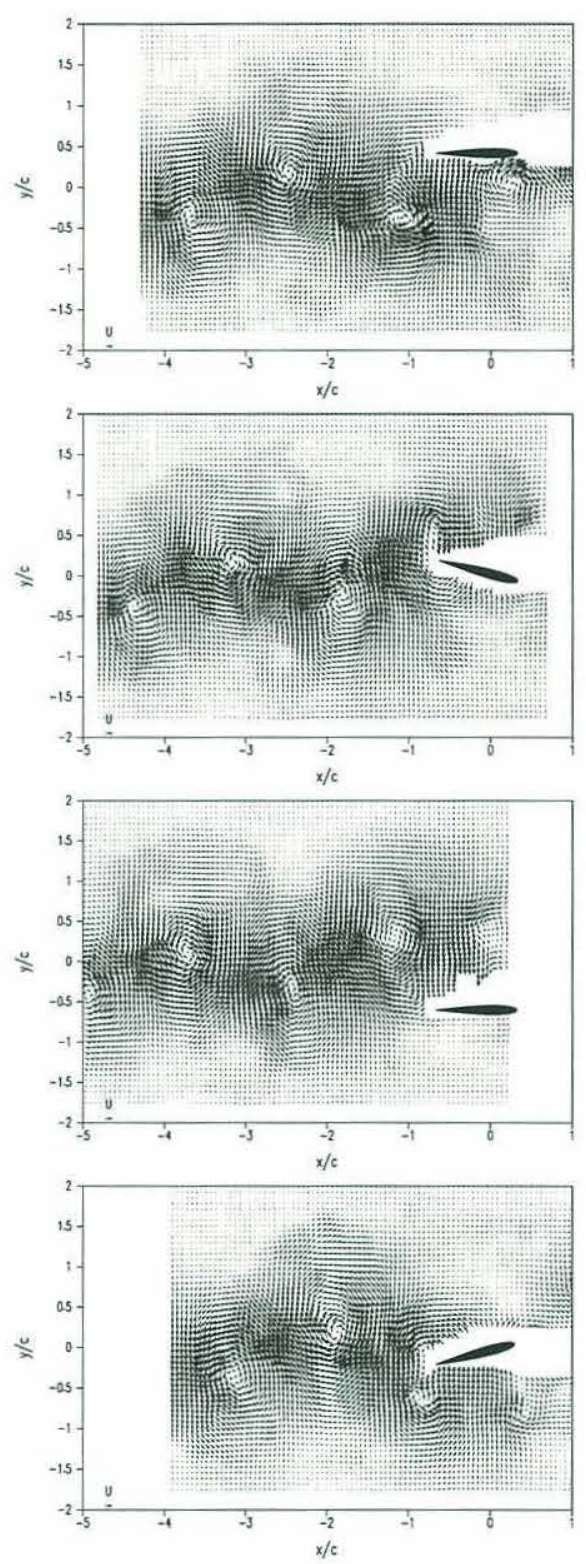
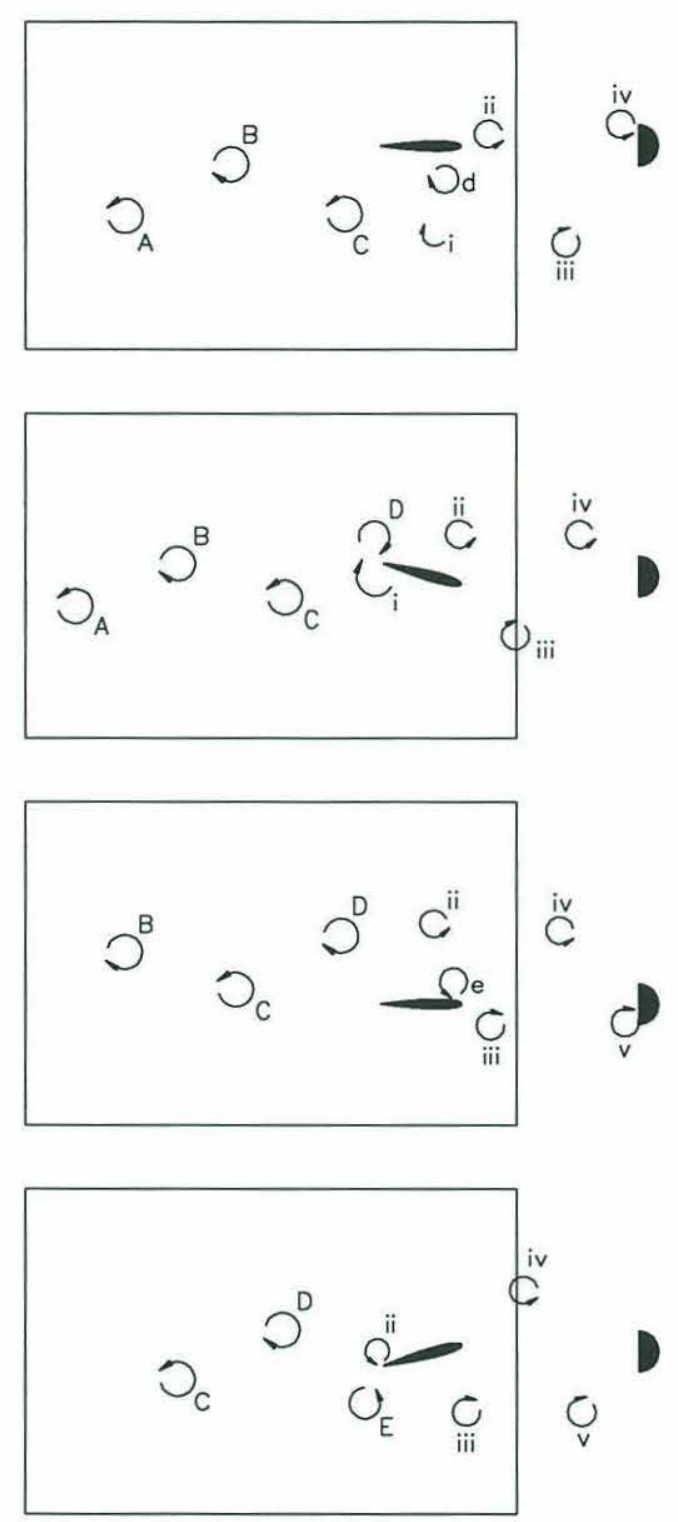

Figure 5-19: Flow summary for vc10 case, $\frac{\sigma}{\lambda}=1.25$, slalom mode with constructive merging of both rows of cylinder vortices with TEV. Velocity fields at quarter points in the heave cycle are shown in the left column, schematic diagrams of the wake vortices in the right column. The abscissa is adjusted such that the foil pivot point is at $\frac{x}{c}=0$. 

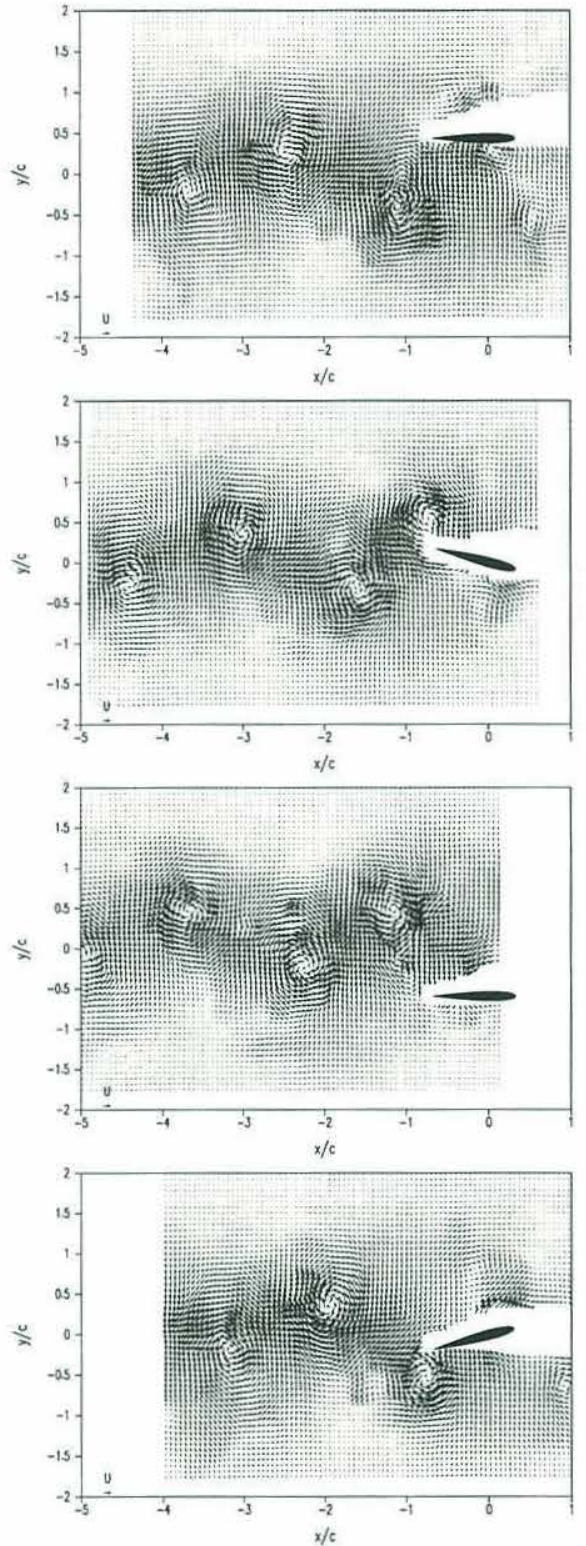
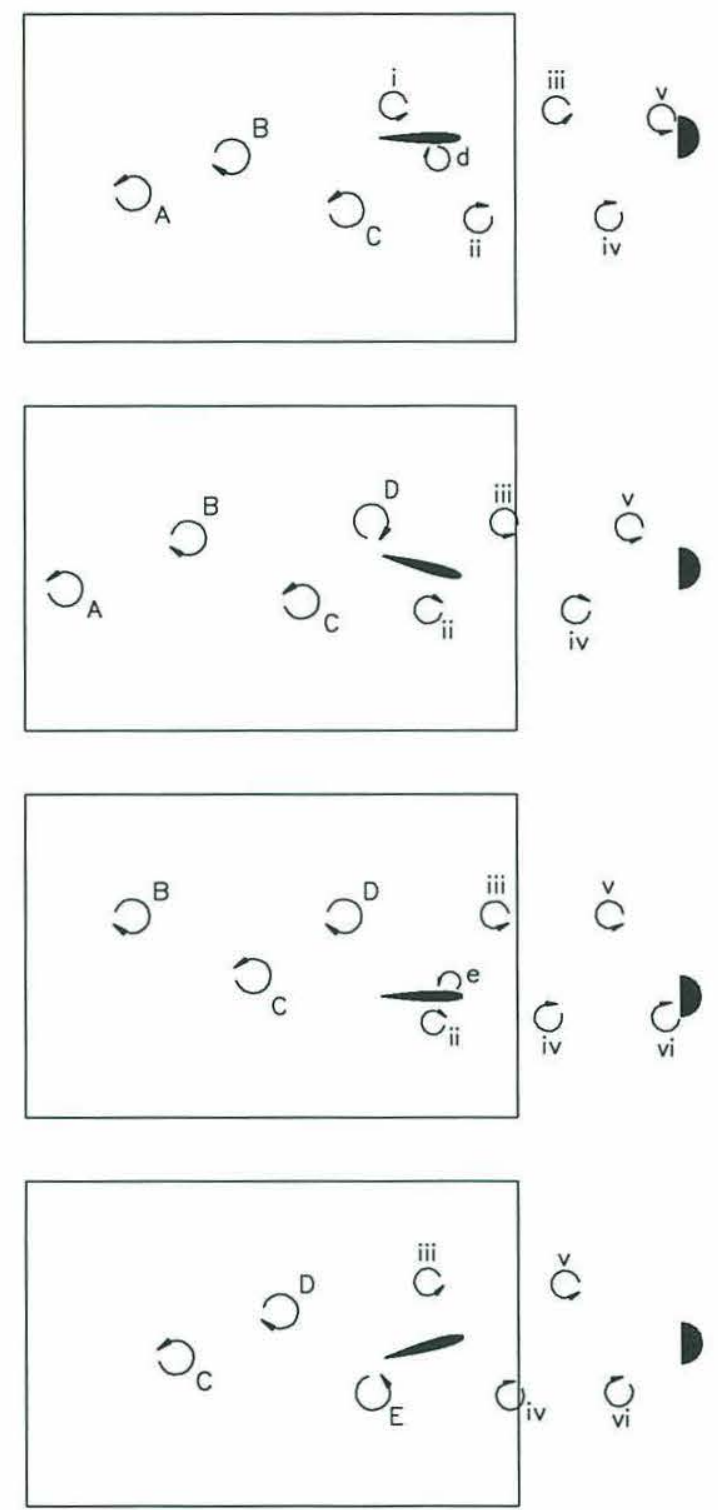

Figure 5-20: Flow summary for vc11 case, $\frac{\sigma}{\lambda}=1.50$, combination interception and slalom mode with annihilation by stretching of both rows of cylinder vortices. Velocity fields at quarter points in the heave cycle are shown in the left column, schematic diagrams of the wake vortices in the right column. The abscissa is adjusted such that the foil pivot point is at $\frac{x}{c}=0$. 

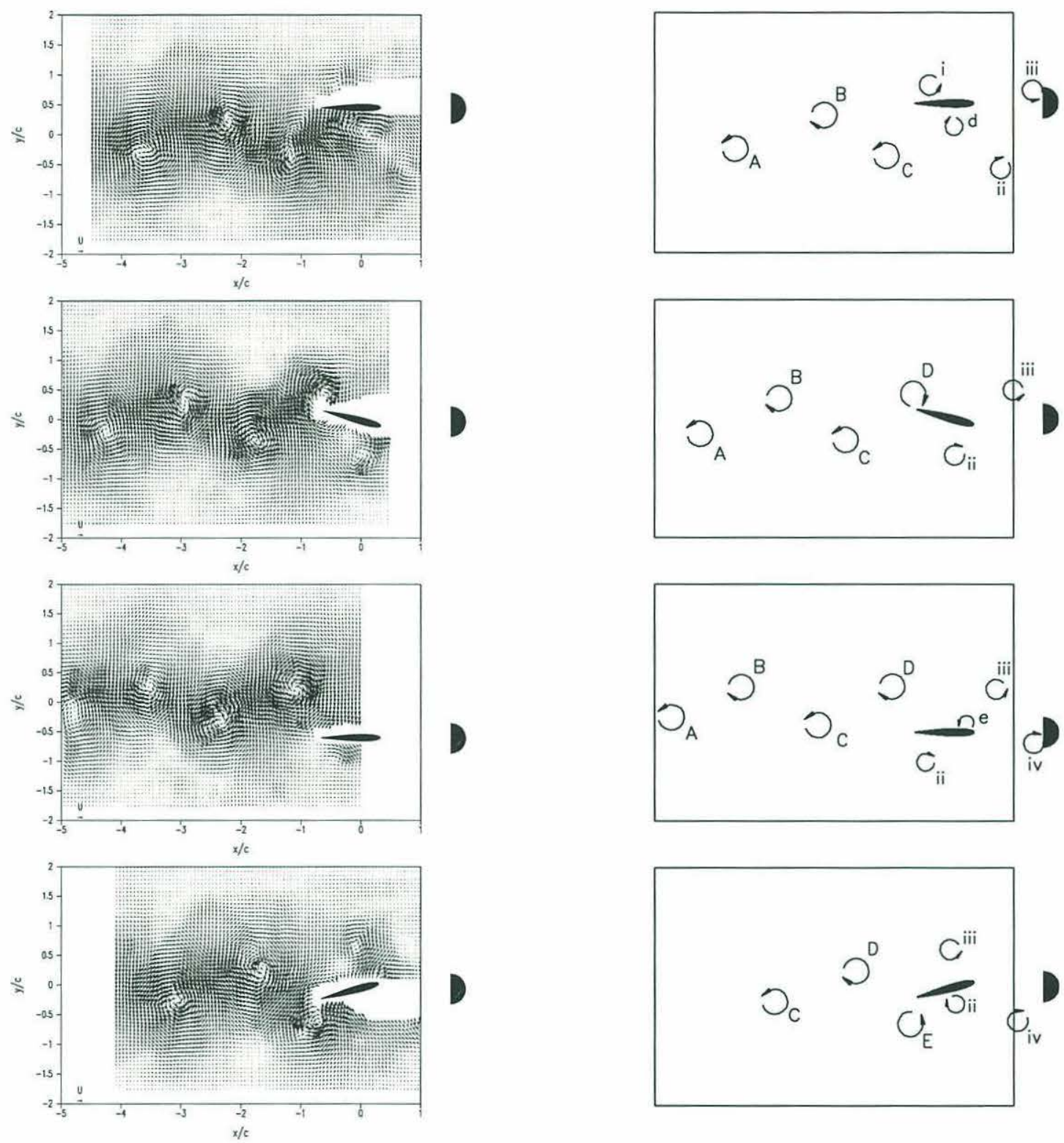

Figure 5-21: Flow summary for vc12 case, $\frac{\sigma}{\lambda}=0.75$, interception mode with destruction of top row vortices by stretching and constructive merging of bottom row vortices with the DSV. Velocity fields at quarter points in the heave cycle are shown in the left column, schematic diagrams of the wake vortices in the right column. The abscissa is adjusted such that the foil pivot point is at $\frac{x}{c}=0$. 
For $\frac{\sigma}{\lambda}=1.00$, both foil motions have essentially the same net thrust ratio, yet the wake interactions are distinctly different. In the lower angle of attack case, the cylinder vortices disintegrate after coming in contact with the foil whereas in the higher angle of attack case, the cylinder vortices join the stall vortex and constructively merge into the trailing edge vorticity. It is unexpected that these two different wake interactions produce the same net result. It would be beneficial to repeat these cases with actual force measurements to ascertain the efficiency of these interactions.

At $\frac{\sigma}{\lambda}=1.50$ the apparent trend for the two experimental groups diverges. The thrust ratio for the lower angle of attack case rises dramatically to its highest value while the higher angle of attack case continues to drop. The difference between these two cases is the interaction with the top row vortices. In case vc5, the cylinder vortex merges constructively with the TEV whereas in vc11 it is annihilated. The force ratio discrepancy is greater than three times the standard deviation of the force coefficient calculation for these cases. Thus, the thrust ratio discrepancy is likely due to the different wake interactions rather than the measurement error.

In each of the interaction cases studied, the wake vortex strength increases in comparison to the tare case of the foil alone. We expect this result because of the increase in angle of attack due to the cylinder deficit wake. Despite the increase in wake vortex strength, the thrust coefficient as computed using momentum methods is always less than the tare value. This contradicts the result given by Gopalkrishnan et al. [38] that the net thrust increases for similar tandem arrangements at lower heave amplitudes and angles of attack.

\subsection{Conclusions}

In this chapter we have investigated the interaction of thrust producing flapping foils with upstream discrete vorticity. Vortex generation by an oscillating D-section cylinder was studied in detail over a wide range of frequencies and amplitudes. Excellent performance was achieved for moderate heave amplitudes $\left(1<\frac{2 A}{d}<3\right)$ and frequencies near the natural cylinder shedding frequency.

We experimentally surveyed the interaction between the oscillating D-cylinder wake and two moderate amplitude, high angle of attack flapping foil motions. The phase of the interaction was adjusted between 0.5 and 1.5 wavelengths by varying the separation between 


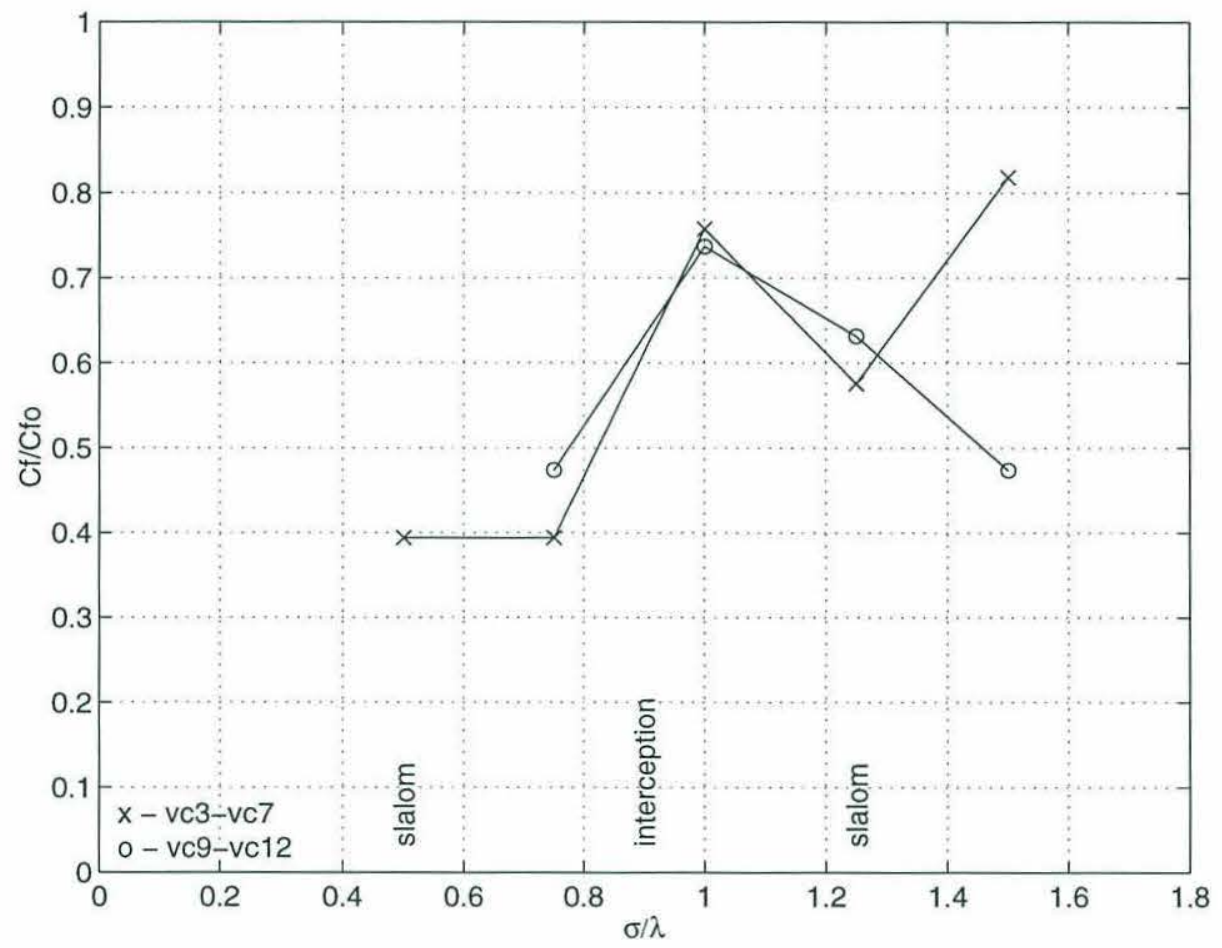

Figure 5-22: Force coefficient variation with interaction mode. Force coefficients are normalized with respect to the corresponding nominal foil alone case (vc3 through vc7 are normalized with $C_{f}($ vc2 $)$ and vc9 through vc12 with $C_{f}($ vc8)). 
the cylinder and foil sections. In all cases, the foil restructures the cylinder wake and replaces it with a reverse Kármán street which is usually narrower than in the nominal foil alone case. The cylinder deficit wake acts to strengthen the effect of the foil by effectively increasing the angle of attack. Hence, the circulation of the wake vortices increases compared to the nominal case.

We observed a variety of wake interactions which depend on the encounter phase of the foil and the cylinder vortices, as well as the foil kinematics. In the lower angle of attack foil cases the following interactions were observed:

- Interception mode (near encounters) $\Longrightarrow$ annihilation by straining

- Slalom mode (avoidance) $\Longrightarrow$ constructive merging with TEV

For the high angle of attack foil motions, the interactions are:

- Interception mode $\Longrightarrow$ constructive merging with DSV, annihilation by straining

- Slalom mode $\Longrightarrow$ constructive merging with TEV

The thrust force is maximum in the interception modes where the foil is heavily loaded. Streitlien [113] observed the same phenomenon his nonlinear simulations with a corresponding increase in efficiency.

In this chapter we have taken the work of Gopalkrishnan and Streitlien one step further. Here we have considered large amplitude, large angle of attack flapping foil motions and the interactions with discrete vortices. Force measurements are required in order to draw conclusions on the efficiency of these motions. 


\section{Chapter 6}

\section{Conclusions}

\subsection{Summary}

In this thesis we have explored propulsion hydrodynamics as a concept in vorticity control. Vortex generation is inherent to jet production and plays a fundamental role in the wake dynamics. Propulsion strategies which exploit the nature of the vortical wake can produce large thrust levels while maintaining high efficiency.

Our experiments with live swimming fish indicate that the undulatory motions of fish are organized such that they lay the foundation for a wake comprised of discrete vortices. Vortices are fundamental to the near wake in fish swimming, rather than a consequence of shear layer roll-up in the wake. During maneuvering the body effect is amplified: the vigorous body contortion during the preparatory stroke initiates the formation of a vortex ring jet which is shed during the main propulsive stroke, imparting momentum to the fluid in the opposite direction of the fish motion.

In flapping foil propulsion, the wake structure depends strongly on foil kinematics. Our exploration of large amplitude flapping foil motions revealed the unexpected result: trailing edge shedding is significant only for low amplitude oscillations. For large amplitude

motions $\left(\frac{2 A}{c} \geq 1.0\right)$, dynamic stall of the foil significantly influences the structure of the wake. The stall vortex was observed to merge with same signed trailing edge vorticity or more commonly, it leaves the foil surface without any apparent trailing edge shedding. The phase relationship between heave and pitch motions greatly influences the stall formation and convection which substantially affects the efficiency. Despite the large quantity of published work in aerodynamics on dynamic stall of wings, virtually all of our results for 
flapping foils are novel contributions.

Our experiments in vorticity control of tandem vortical wakes demonstrate that a strong drag wake can be effectively cancelled by operating a thrust producing foil in the wake. The nature of the foil interaction with upstream vorticity depends on the proximity (phase) of the encounter and the foil kinematics. Upstream vortices either strain to destruction or convectively merge with leading or trailing edge foil vorticity. These vorticity control experiments provide proof of concept that a flapping foil can effectively manipulate upstream vorticity.

\subsection{Recommendations for further research}

Although DPIV has proved to be a powerful and effective measurement technique, there are several issues yet to be resolved. The uppermost concern in these types of experiments is obtaining the flow very near to a solid surface. In our experiments we have taken the straightforward approach of masking the data such that we only interpret the real flow and not spurious data caused by shadows, reflections and the image of the solid object. We have yet to undertake the combined method of particle tracking and particle image velocimetry near moving bodies because in our own experiments, our typical fluid boundaries move and deform, sometimes unpredictably as in the case of a swimming fish. For the method to be generally applicable, considerable effort must be expended to develop the image processing algorithms to handle complex, arbitrary geometries. Advances in the method would be immediately applicable to a variety of bounded flows including flows near the free surface as well as solid and moving boundaries.

Once the flow near the body is fully revealed, the instantaneous force the body imparts on the fluid can be calculated elegantly using the vorticity field. Wu [139] presents a method for computing the viscous force on the fluid based on the time rate of change of the first moment of vorticity and the contribution from solid body rotation. The method looks especially promising in the case of flapping foils since the kinematics are precisely known.

On the topic of vorticity control strategies, force and efficiency measurements are needed to synthesize our wake interaction observations. We were only able to measure the net wake strength (thrust coefficient and vortex circulation) of our tandem arrangements. In some cases, markedly different interactions resulted in similar wake strength. Likely, the efficiency 
varies considerably. For instance, capture of the cylinder wake vortices which merge with the foil stall vortex elegantly redirects drag energy to foil thrust enhancement, probably with increased efficiency. Our experiments were limited to heavily loaded foil motions. Further study of lightly loaded (low angle of attack) motions may reveal other interesting wake interactions. 


\section{References}

[1] I.H. Abbott and A.E. Von Doenhoff. Theory of Wing Sections. Dover Publications, Inc., 1959.

[2] R.J. Adrian. Multi-point optical measurements of simultaneous vectors in unsteady flow-a review. Int. Journal Heat Fluid Flow, 7:127-145, 1986.

[3] A.R. Ahmadi and S.E. Widnall. Energetics and optimum motion of oscillating lifting surfaces. AIAA Paper 83-1710, pages 1-18, 1983.

[4] A.R. Ahmadi and S.E. Widnall. Energetics of oscillating lifting surfaces by the use of integral conservation laws. Journal of Fluid Mechanics, 266:347-370, 1994.

[5] R.D. Archer, J. Sapuppo, and D.S. Betteridge. Propulsion characteristics of flapping wings. Aeronautical Journal, pages 355-371, September 1979.

[6] Hassan Aref. Integrable, chaotic, and turbulent vortex motion in two-dimensional flows. Annual Review of Fluid Mechanics, 15:345-389, 1983.

[7] G.K. Batchelor. An Introduction to Fluid Dynamics. Cambridge University Press, 1967.

[8] P.W. Bearman. Vortex shedding from oscillating bluff bodies. Annual Review of Fluid Mechanics, 16:195-222, 1984.

[9] A.J. Bilanin, M.E. Teske, and G.G. Williamson. Vortex interactions and decay in aircraft wakes. AIAA Journal, 15(2):250-260, 1977.

[10] R.W. Blake. Fish Locomotion. Cambridge University Press, Cambridge, 1983.

[11] R.D. Blevins. Flow-Induced Vibration. Van Nostrand Reinhold Company, New York, NY, 1990.

[12] E.R. Booth Jr. Measurement of velocity and vorticity fields in the wake of an airfoil in periodic pitching motion. NASA Technical Paper 2780, 1987.

[13] E.R. Booth Jr. Experimental observations of two-dimensional blade-vortex interaction. AIAA Journal, 28(8):1353-1359, 1990.

[14] E.R. Booth Jr. and J.C. Yu. Two-dimensional blade-vortex flow visualization investigation. AIAA Journal, 24(9):1468-1473, 1986.

[15] N. Bose and J. Lien. Propulsion of a fin whale (balaenoptera physalus): why the fin whale is a fast swimmer. Proc. R. Soc. Lond. B, 237:175-200, 1989. 
[16] N. Bose and J. Lien. Energy absorption from ocean waves: a free ride for cetaceans. Proc. R. Soc. London B, 240:591-605, 1990.

[17] J.B. Bratt. Flow patterns in the wake of an oscillating aerofoil. Aeronautical Research Council Reports and Memoranda No. 2773, pages 1-28, 1953.

[18] C.M. Breder. The locomotion of fishes. Zoologica, 4:159-256, 1926.

[19] J.-Y. Cheng, L.-X. Zhuang, and B.-G. Tong. Analysis of swimming three-dimensional waving plates. Journal of Fluid Mechanics, 232:341-355, 1991.

[20] M.G. Chopra. Hydrodynamics of lunate-tail swimming propulsion. Journal of Fluid Mechanics, 64:375-391, 1974.

[21] M.G. Chopra. Large amplitude lunate-tail theory of fish locomotion. Journal of Fluid Mechanics, 74:161-182, 1976.

[22] M.G. Chopra and T. Kambe. Hydromechanics of lunate-tail swimming propulsion. Part 2. Journal of Fluid Mechanics, 79:49-69, 1977.

[23] J.P. Comstock. Principles of Naval Architecture. The Society of Naval Architects and Marine Engineers, New York, NY, 1967.

[24] J. Cox. The revolutionary Kasper wing. Soaring, pages 20-23, December 1973.

[25] M.E. Davies. A comparison of the wake structure of a stationary and oscillating bluff body, using a conditional averaging technique (part 2). Journal of Fluid Mechanics, 75:209-231, 1976.

[26] J.D. DeLaurier and J.M. Harris. Experimental study of oscillating-wing propulsion. Journal of Aircraft, 19(5):368-373, 1982.

[27] H. Dewar and J.B. Graham. Studies of tropical tuna swimming performance in a large water tunnel. Journal of Experimental Biology, 192:13-31, 1994.

[28] T.L. Doligalski, C.R. Smith, and J.D.A. Walker. Vortex interactions with walls. Annual Review of Fluid Mechanics, 26:573-616, 1994.

[29] L.L. Van Dommelen. Lagrangian techniques for unsteady flow separation. In Forum on Unsteady Flow Separation, Proceedings of the 1987 ASME Applied Mechanics, Bioengineering, and Fluids Engineering Conference, volume 52, pages 81-84, New York, 1987. American Society of Mechanical Engineers.

[30] David G. Dritschel. The nonlinear evolution of rotating configurations of uniform vorticity. Journal of Fluid Mechanics, 172:157-182, 1986.

[31] H.L. Fierstine and V. Walters. Studies in locomotion and anatomy of scombrid fishes. Memoir of the Southern California Academy of Sciences, 6:1-31, 1968.

[32] Peter Freymuth. Propulsive vortical signature of plunging and pitching airfoils. AIAA Journal, 26(7):881-883, 1988.

[33] Peter Freymuth. Thrust generation by an airfoil in hover modes. Experiments in Fluids, 9:17-24, 1990. 
[34] M. Gad-el-Hak and D.M. Bushnell. Separation control: review. Journal of Fluids Engineering, 113:5-30, 1991.

[35] M. Gad-el-Hak and C.-M. Ho. Unsteady vortical flow around three-dimensional lifting surfaces. AIAA Journal, 24(5):713-721, 1986.

[36] C.P. Gendrich, M.M. Koochesfahani, and M.R. Visbal. Effects of initial acceleration on the flow field development around rapidly pitching airfoils. Journal of Fluids Engineering, 117:45-49, 1995.

[37] M. Gharib and C. Willert. Advances in Fluid Mechanics, volume 45 of Lecture Notes in Engineering, chapter Particle Tracing: Revisited, pages 109-199. Springer-Verlag, editor M. Gad-el-Hak, 1989.

[38] R. Gopalkrishnan, M.S. Triantafyllou, G.S. Triantafyllou, and D. Barrett. Active vorticity control in a shear flow using a flapping foil. Journal of Fluid Mechanics, 274:1-21, 1994.

[39] Ramnarayan Gopalkrishnan. Vortex-induced forces on oscillating bluff cylinders. PhD thesis, Massachusetts Institute of Technology/Woods Hole Oceanographic Institution Joint Program, Cambridge/Woods Hole, MA, 1993.

[40] M. Gorman and H.C. Swinney. Spatial and temporal characteristics of modulated waves in the circular Couette system. Journal of Fluid Mechanics, 117:123-142, 1982.

[41] J. Gray. Studies in animal locomotion VI. the propulsive powers of the dolphin. Journal of Experimental Biology, 13:192-199, 1936.

[42] James Gray. Animal Locomotion. Weidenfield and Nicolson, London, 1968.

[43] J. Grue, A. Mo, and E. Palm. Propulsion of a foil moving in water waves. Journal of Fluid Mechanics, 186:393-417, 1988.

[44] I. Gursul and C.-M. Ho. High aerodynamic loads on an airfoil submerged in an unsteady stream. AIAA Journal, 30(4):1117-119, 1992.

[45] I. Gursul, H. Lin, and C.-M. Ho. Vorticity dynamics of 2-D and 3-D wings in unsteady free stream. AIAA Paper 91-0010, pages 1-7, 1991.

[46] D.G. Harper and R.W. Blake. Fast-start performance of rainbow trout salmo gairdneri and northern pike esox lucius. Journal of Experimental Biology, 150:321-342, 1990.

[47] Sighard F. Hoerner. Fluid-Dynamic Drag. published by the author, 1965.

[48] H. Honji and S. Tandeda. Vortex wakes of oscillating circular cylinders. Reports of Research Institute for Applied Mechanics (Kyushu University, Japan), 16(54):211-222, 1968.

[49] Elizabeth Ann Horwich. Unsteady response of a two-dimensional hydrofoil subject to high reduced frequency gust loading. Master's thesis, Massachusetts Institute of Technology, 1993.

[50] M.-K. Huang and C.-Y. Chow. Trapping of a free vortex by Joukowski airfoils. AIAA Journal, 20(1):292-298, 1982. 
[51] H. Isshiki and M. Murakami. A theory of wave devouring propulsion (4th report). Journal of the Society of Naval Architects of Japan, 156:102-114, 1984.

[52] G.E. Karniadakis and G.S. Triantafyllou. Frequency selection and asymptotic states in laminar wakes. Journal of Fluid Mechanics, 199:441-469, 1989.

[53] G. Karpouzian, G. Spedding, and H.K. Cheng. Lunate-tail swimming propulsion. Part 2. Performance analysis. Journal of Fluid Mechanics, 210:329-351, 1990.

[54] J. Katz and D. Weihs. Behavior of vortex wakes from oscillating airfoils. Journal of Aircraft, 15(1):861-863, 1978.

[55] J. Katz and D. Weihs. Hydrodynamic propulsion by large amplitude oscillation of an airfoil with chordwise flexibility. Journal of Fluid Mechanics, 88:485-497, 1978.

[56] J. Katz and D. Weihs. Large amplitude unsteady motion of a flexible slender propulsor. Journal of Fluid Mechanics, 90(4):713-723, 1979.

[57] Manoochehr M. Koochesfahani. Vortical patterns in the wake of an oscillating airfoil. AIAA Journal, 27(9):1200-1205, 1989.

[58] Manoochehr M. Koochesfahani. personal communication, October 1995.

[59] M.M. Koochesfahani and P.E. Dimotakis. A cancellation experiment in a forced turbulent shear layer. 88-3713-CP, 1988.

[60] C. Krick, R. Blickhan, and W. Nachtigall. Architektur und Energetik der Wirbelstrasse frei schwimmender Fische. Verhandlungen Deutsche Zoologische Gesellschaft, 87(1):101, 1994.

[61] P.S.K. Lai, N. Bose, and R.C. McGregor. Wave propulsion from a flexible-armed, rigid-foil propulsor. Marine Technology, 30(1):30-38, 1993.

[62] M.J. Lighthill. Note on the swimming of slender fish. Journal of Fluid Mechanics, 9:305-317, 1960.

[63] M.J. Lighthill. Aquatic animal propulsion of high hydrodynamic efficiency. Journal of Fluid Mechanics, 44:265-301, 1970.

[64] Sir James Lighthill. Mathematical Biofluiddynamics. Society for Industrial and Applied Mathematics, Philadelphia, Pennsylvania, 1975.

[65] L. Lourenco and A. Krothapalli. On the accuracy of velocity and vorticity measurements with PIV. Experiments in Fluids, 18:421-428, 1995.

[66] John J. Magnuson. Fish Physiology, Vol. VII, chapter 4. Locomotion by scombrid fishes: hydromechanics, morphology, and behavior. Academic Press, 1978.

[67] P. Matisse and M. Gorman. Neutrally buoyant anisotropic particles for flow visualization. The Physics of Fluids, 27(4):759-760, 1984.

[68] K.W. McAlister, L.W. Carr, and W.J. McCroskey. Dynamic stall experiments on the NACA 0012 airfoil. Technical Paper 1100, NASA, January 1978. 
[69] W.J. McCroskey. Unsteady airfoils. Annual Review of Fluid Mechanics, 14:285-311, 1982.

[70] W.J. McCroskey and S.L. Pucci. Viscous-inviscid interaction on oscillating airfoils. AIAA Paper 81-0051, pages 1-16, 1981.

[71] C.W. McCutchen. Flow visualization with stereo shadowgraphs of stratified flow. Journal of Experimental Biology, 65:11-20, 1976.

[72] U.B. Mehta. Dynamic stall of an oscillating airfoil. AGARD-CP-227, 1978. Paper 23.

[73] G.E.A. Meier and R. Timm. Unsteady vortex airfoil interaction. AGARD-CP-386, pages $16-1-16-10$.

[74] Scott Nielsen Miller. Measurement of vortex-induced oscillations of marine cables using feedback with explicit structural modelling. Master's thesis, Massachusetts Institute of Technology, Cambridge, MA, 1996.

[75] L.M. Milne-Thomson. Theoretical Hydrodynamics. The Macmillan Company, New York, 1960.

[76] D.T. Mook and B. Dong. Perspective: numerical simulations of wakes and bladevortex interaction. Journal of Fluids Engineering, 116:5-21, 1994.

[77] B.R. Morton. The generation and decay of vorticity. Geophys. Astrophys. Fluid Dynamics, 28:227-308, 1984.

[78] T. Nakaoaka and Y. Toda. Laminar flow computation of fish-like motion wing. In Proceedings of the Fourth (1994) International Offshore and Polar Engineering Conference, Osaka, Japan, volume III, pages 530-538. The International Society of Offshore and Polar Engineers, April 1994.

[79] J.N. Newman. Marine Hydrodynamics. The MIT Press, Cambridge, MA, 1977.

[80] J.N. Newman and T.Y. Wu. Swimming and Flying in Nature, chapter Hydromechanical aspects of fish swimming. Plenum Press, 1975.

[81] H. Oertel, Jr. Wakes behind blunt bodies. Annual Review of Fluid Mechanics, 22:539$564,1990$.

[82] K. Ohmi, M. Coutanceau, O. Daube, and T.P. Loc. Further experiments on vortex formation around an oscillating and translating airfoil at large incidences. Journal of Fluid Mechanics, 225:607-630, 1991.

[83] K. Ohmi, M. Coutanceau, T.P. Loc, and A. Dulieu. Vortex formation around an oscillating and translating at large incidences. Journal of Fluid Mechanics, 211:37$60,1990$.

[84] M.F.M. Osborne. Aerodynamics of flapping flight with application to insects. Journal of Experimental Biology, 28(2):221-245, 1951.

[85] Y. Oshima and A. Natsume. Flow field around an oscillating airfoil. In W. Merzkirch, editor, Flow Visualization II, Proceedings of the Second International Symposium on Flow Visualization, pages 295-299, Bochum, Germany, 1980. Hemisphere Publishing Corporation. 
[86] Y. Oshima and K. Oshima. Vortical flow behind an oscillating airfoil. In Proceedings of the 15th International Congress, pages 357-368. International Union of Theoretical and Applied Mechanics, 1980.

[87] M. P. Paidoussis. Hydroelastic ichthyoid propulsion. Journal of Hydronautics, 10(1):30-32, 1976.

[88] J. Panda and K.B.M.Q. Zaman. Experimental investigation of the flow field of an oscillating airfoil and estimation of lift from wake surveys. Journal of Fluid Mechanics, 265:65-95, 1994.

[89] S.O. Park, J.S. Kim, and B.I. Lee. Hot-wire measurements of near wakes behind an oscillating airfoil. AIAA Paper 88-3715-CP, pages 1217-1223, 1988.

[90] D.R. Poling and D.P. Telionis. The response of airfoils to periodic disturbances-the unsteady Kutta condition. AIAA Journal, 24(2):193-199, 1986.

[91] Ludwig Prandtl. Essentials of Fluid Dynamics. Hafner Publishing Company, New York, 1952.

[92] W.C. Reynolds and L.W. Carr. Review of unsteady, driven, separated flows. AIAA Paper 85-0527, 1985.

[93] M.C. Robinson, H.E. Helin, and M.W. Luttges. Control of wake structure behind an oscillating airfoil. AIAA Paper 86-2282, pages 472-485, 1986.

[94] M.C. Robinson and J.B. Wissler. Pitch rate and reynolds number effects on a pitching rectangular wing. AIAA Paper 88-2577-CP, 1988.

[95] L.C. Rome, A. Sosnicki, and I.-H. Choi. The influence of temperature on muscle function in the fast swimming scup. Journal of Experimental Biology, 163:281-295, 1992.

[96] Moe William Rosen. Water flow about a swimming fish. Master's thesis, University of California, Los Angeles, China Lake, CA, 1959.

[97] Moe William Rosen. Experiments with swimming fish and dolphins. ASME Paper 61-WA-203, 1961.

[98] P.G. Saffman. Dynamics of vorticity. Journal of Fluid Mechanics, 106:49-58, 1981.

[99] P.G. Saffman and G.R. Baker. Vortex interactions. Annual Review of Fluid Mechanics, 11:95-122, 1979.

[100] P.G. Saffman and J.C. Schatzman. Stability of a vortex street of finite vortices. Journal of Fluid Mechanics, 117:171-185, 1982.

[101] P.G. Saffman and J.S. Sheffield. Flow over a wing with an attached free vortex. Studies in Applied Mathematics, 57:107-117, 1977.

[102] P.G. Saffman and R. Szeto. Equilibrium shapes of a pair of equal uniform vortices. Physics of Fluids, 23(12):2339-2342, 1980. 
[103] T. Sarpkaya. Vortex-induced oscillations, a selective review. Journal of Applied Mechanics, 46:241-258, 1979.

[104] Ö. Savaş. On flow visualization using reflective flakes. Journal of Fluid Mechanics, $152: 235-248,1985$.

[105] J.O. Scherer. Experimental and theoretical investigation of large amplitude oscillating foil propulsion systems. Technical Report 662-1, U.S. Army Engineering Research and Development Laboratories, Hydronautics, Inc., Laurel, Maryland, May 1968.

[106] A. Shekarriz, T.C. Fu, and J. Katz. Near-field behavior of a tip vortex. AIAA Journal, 31(1):112-118, 1993.

[107] C. Shih, L. Lourenco, L. Van Dommelen, and A. Krothapalli. Unsteady flow past an airfoil pitching at constant rate. AIAA Journal, 30(5):1153-1161, 1992.

[108] J. Siekmann. Theoretical studies of sea animal locomotion, part 1. Ing. Archiv, $31: 214-228,1962$.

[109] J. Siekmann. Theoretical studies of sea animal locomotion, part 2. Ing. Archiv, $32: 40-50,1963$.

[110] J.E.L. Simmons. Phase-angle measurements between hot-wire signals in the turbulent wake of a two-dimensional bluff body. Journal of Fluid Mechanics, 64:599-609, 1974.

[111] P.K. Stansby. The locking-on of vortex shedding due to the cross-stream vibration of circular cylinders in uniform and shear flows. Journal of Fluid Mechanics, 74:641-665, 1976.

[112] K. Streitlien and M.S. Triantafyllou. Force and moment on a Joukowski profile in the presence of point vortices. AIAA Journal, 33(4):603-610, 1995.

[113] Knut Streitlien. Extracting Energy from Unsteady Flows through Vortex Control. PhD thesis, Massachusetts Institute of Technology/Woods Hole Oceanographic Institution Joint Program, Cambridge/Woods Hole, MA, 1994.

[114] J. Świrydczuk. A visualization study of the interaction of a free vortex with the wake behind an airfoil. Experiments in Fluids, 9:181-190, 1990.

[115] S. Taneda and Y. Tomonari. An experiment on the flow around a waving plate. Journal of the Physical Society of Japan, 36(6):1683-1689, 1974.

[116] Sadatoshi Taneda. Experimental investigation of vortex streets. Journal of the Physical Society of Japan, 20(9):1714-1721, 1965.

[117] Sadatoshi Taneda. Visual study of unsteady separated flows around bodies. Prog. Aerospace Science, 17:287-348, 1977.

[118] G.S. Triantafyllou, M.S. Triantafyllou, and M.A. Grosenbaugh. Optimal thrust development in oscillating foils with application to fish propulsion. Journal of Fluids and Structures, 7:205-224, 1993.

[119] M.S. Triantafyllou, D.S. Barrett, K. Streitlien, and J.M. Anderson. Flow patterns and propulsive efficiency of harmonically flapping foils. (in preparation), 1996. 
[120] M.S. Triantafyllou, G.S. Triantafyllou, and R. Gopalkrishnan. Wake mechanics for thrust generation in oscillating foils. Physics of Fluids A, 3(12):2835-2837, 1991.

[121] M. Van Dyke. An Album of Fluid Motion. The Parabolic Press, Stanford, California, 1982.

[122] J.J. Videler and F. Hess. Fast continuous swimming of two pelagic predators, saithe (pollachius virens) and mackerel (scomber scombrus): a kinematic analysis. Journal of Experimental Biology, 109:209-228, 1984.

[123] John J. Videler. Fish Swimming. Chapman \& Hall, 1993.

[124] T.A. Videv and Y. Doi. Numerical study of the flow and thrust produced by a pitching 2D hydrofoil. Journal of the Society of Naval Architects of Japan, 172:165-174, 1992.

[125] M.R. Visbal and J.S. Shang. Investigation of the flow structure around a rapidly pitching airfoil. AIAA Journal, 27(8):1044-1051, 1989.

[126] T. von Kármán and J.M. Burgers. General Aerodynamic Theory: Perfect Fluids, volume 2 of Aerodynamic Theory. Springer, Leipzig, 1934.

[127] V. Walters. Body form and swimming performance in the scombroid fishes. American Zoologist, 2:143-149, 1962.

[128] Paul W. Webb. Is the high cost of body/caudal fin undulatory swimming due to increased frictional drag or inertial recoil? Journal of Experimental Biology, 162:157$166,1992$.

[129] P.W. Webb. The effect of size on the fast-start performance of rainbow trout salmo gairdneri, and a consideration of piscivorous predator-prey interactions. Journal of Experimental Biology, 65:157-177, 1976.

[130] P.W. Webb. Fast-start performance and body form in seven species of teleost fish. Journal of Experimental Biology, 74:211-226, 1978.

[131] D. Weihs. A hydrodynamic analysis of fish turning manoeuvres. Proc. Royal Soc. London B, 182:59-72, 1972 .

[132] D. Weihs. The mechanism of rapid starting in slender fish. Biorheology, 10:343-350, 1973.

[133] D. Weihs. On the existence of multiple Kármán vortex-street modes. Journal of Fluid Mechanics, 61:199-205, 1973.

[134] Sheila E. Widnall. The structure and dynamics of vortex filaments. Annual Review of Fluid Mechanics, 7:141-165, 1975.

[135] C.E. Willert. The interaction of modulated vortex pairs with a free surface. $\mathrm{PhD}$ thesis, University of California, San Diego, 1992.

[136] C.E. Willert and M. Gharib. Digital particle image velocimetry. Experiments in Fluids, 10:181-193, 1991. 
[137] C.E. Willert and M. Gharib. Three-dimensional particle imaging with a single camera. Experiments in Fluids, 12:353-358, 1992.

[138] C.H.K Williamson and A. Roshko. Vortex formation in the wake of an oscillating cylinder. Journal of Fluids and Structures, 2:355-381, 1988.

[139] J.C. Wu. Theory for aerodynamic force and moment in viscous flows. AIAA Journal, 19(4):432-441, 1980.

[140] J.Z. Wu, A.D. Vakili, and J.M. Wu. Review of the physics of enhancing vortex lift by unsteady excitation. Prog. Aerospace Sci., 28:73-131, 1991.

[141] T.Y. Wu. Swimming of a waving plate. Journal of Fluid Mechanics, 10:321-344, 1961.

[142] T.Y. Wu. Hydromechanics of swimming propulsion. Part 1. Swimming of a twodimensional flexible plate at variable forward speeds in an inviscid fluid. Journal of Fluid Mechanics, 46:337-355, 1971.

[143] T.Y. Wu. Hydromechanics of swimming propulsion. Part 2. Some optimum shape problems. Journal of Fluid Mechanics, 46:521-544, 1971.

[144] T.Y. Wu. Hydromechanics of swimming propulsion. Part 3. Swimming and optimum movement of slender fish with side fins. Journal of Fluid Mechanics, 46:545-568, 1971.

[145] H. Yamaguchi and N. Bose. Oscillating foils for marine propulsion. In Proceedings of the Fourth (1994) International Offshore and Polar Engineering Conference, Osaka, Japan, volume III, pages 539-544. The International Society of Offshore and Polar Engineers, April 1994.

[146] G.T. Yates. Fish Biomechanics, chapter 6. Hydromechanics of body and caudal fin propulsion, pages 177-213. Praeger, New York, 1983.

[147] M.M. Zdravkovich. Review and classification of various aerodynamic and hydrodynamic means for suppressing vortex shedding. Journal of Wind Engineering and Industrial Aerodynamics, 7:145-189, 1981.

[148] M.M. Zdravkovich. Modification of vortex shedding in the synchronization range. Journal of Fluids Engineering, 104:513-517, December 1982. 